



\title{
ARCHITECTURE AT THE EDGE OF CHAOS
}

a model for uncertainty in architecture

\author{
by \\ Calvin Fung \\ B.Arch.Sc., Ryerson University, Toronto, 2010 \\ A thesis \\ presented to Ryerson University \\ in partial fulfillment of the \\ requirements for the degree of \\ Master of Architecture \\ in the Program of \\ Architecture
}

Toronto, Ontario, Canada, 2017

(C) Calvin Fung 2017 



\section{AUTHOR'S DECLARATION}

I hereby declare that I am the sole author of this thesis. This is a true copy of the thesis, including any required final revisions, as accepted by my examiners.

I authorize Ryerson University to lend this thesis to other institutions or individuals for the purpose of scholarly research.

I further authorize Ryerson University to reproduce this thesis by photocopying or by other means, in total or in part, at the request of other institutions or individuals for the purpose of scholarly research.

I understand that my thesis may be made electronically available to the public. 



\title{
Architecture at the Edge of Chaos: A Model for Uncertainty in Architecture
}

\author{
Calvin Fung
}

Master of Architecture, 2017

Architecture, Ryerson University

\section{ABSTRACT}

The changing needs of society informed by rapid technological, social and ecological changes have disturbed the foundation of permanence on which much of architecture was built. Traditional Western architecture is too solid, hard, and slow_presenting difficulties for it to adequately adapt to change and uncertainty. A reconceptualization of architecture is necessary, one not focused on the certainty of solutions or forms, but one patterned by the dynamic feedback of human agency and environmental forces. For architecture to adapt, and to adapt to unpredictable circumstances, requires that architecture accept uncertainty in its formulation and materialization. Embracing systems-based thinking, a conceptual model based on the complex systems of granular matter provides a unique approach to architecture's material and immaterial structures. Architecture will then be critically poised at the edge of chaos, ready to reorganize and evolve towards a new fluid paradigm. 



\section{PREFACE}

After trying to address adaptability and change throughout my early architectural projects, and confronting difficult questions concerning designing uncertainty, it was apparent to me that standard architectural materials, practices and thinking were not going to be the solution. What I desired was a "magical" amorphous material: strong yet malleable, freely configurable by its users. It seemed to be the stuff of science fiction. It was during the 2015 spring/summer studio, a studio focused on material investigations that I found what I was looking for. I (re)encountered the special world of aggregate materials as I chose it for further study. The immediate excitement it incited at the time from my brief exposure to it months prior in a seminar should have been a hint.

The early material exploration in aggregation processes were conceived and conducted together with Victor Huynh during the spring/ summer Adaptive Architecture studio. The subsequent reflection and synthesis of ideas that form the basis for this thesis and is an original intellectual product of the author. Following the studio, an early development/form of such ideas has been recognized by the 2015 Laka Competition, and has been publicized online and exhibited in Poland. A print publication is anticipated by the competition organizers.

Several design applications and explorations have been conducted in this thesis through competitions and real projects. Sands of Tomorrow (Design Exploration 1) was conceived with Victor as part of the Adaptive Architecture studio for the Sukkhaville 2015 competition. Flow was an installation designed and constructed also with Victor for the 2016 Winter Station Competition. Its conception was guided by research goals initiated by the author and it forms Design Exploration 2. It has been publicized in various media outlets. Seeds (Design Exploration 3) was a collaboration with the Edible Allan Gardens initiative and saw the integration of the Flow components within the overall garden design. The author oversaw and implemented the development of the seed components. JaXX (Design Exploration 4) was a finalist of the Mako Student Innovation Award and was pitched by the author at a live adjudication event.

Although this thesis is based on several explorations centered on a specific material system, my background is in neither material science nor engineering. As a result, the experiments are more qualitative than quantitative. My main interest in this thesis is in the relationship between matter and the creative possibilities it affords for design and for its users. It is my belief that architects should be equipped with the most diverse range of knowledge. Perhaps it is in spite of such distinction of being outside of material science or engineering that I have the opportunity to take creative leaps and bring to surface insights for architecture and application, without being fully limited by technical obstacles. 



\section{ACKNOWLEDGEMENTS}

My sincerest gratitude is owed to the following:

Dr. June Komisar (supervisor),

a consummate scholar, for keeping an open-mind and taking on this unique thesis with unwavering enthusiasm. I am most grateful for your understanding, courage and the staunch support.

Scott Sørli (second reader),

for sharing your diverse knowledge and the enlightening discussions.

Tom Bessai.

for igniting a passion and interest in this field of research.

Professors Vincent Hui, Arthur Wrigglesworth, Steven Beites, and Filiz Klassen,

the path towards this thesis was shaped by the courses and dialogue we have had during my graduate studies

The MArch cohort,

Victor, for originally entertaining this crazy idea and a particular few for the conversations that kept me going.

Turner Fleischer Architects,

to the management, for your confidence and dedication to education and support for continued learning.

Zohra Akbari,

for being there every single step of the way. 


\section{TABLE OF CONTENTS}

AUTHOR'S DECLARATION iii

ABSTRACT

PREFACE vii

ACKNOWLEDGMENTS $\quad$ ix

LIST OF TABLES $\quad$ xiii

LIST OF FIGURES $\quad$ xiv

LIST OF APPENDICES $\quad$ xxi

$\begin{array}{ll}\text { INTRODUCTION } & 1\end{array}$

The Form of the Thesis $\quad 2$

$\begin{array}{ll}\text { PART 1: BACKGROUND } & 7\end{array}$

$\begin{array}{lc}\text { Firmitas } & 8\end{array}$

$\begin{array}{ll}\text { Lineage } & 10\end{array}$

PART 2: SYSTEMS APPROACH 19

$\begin{array}{ll}\text { Complexity \& The Edge of Chaos } & 20\end{array}$

$\begin{array}{ll}\text { Material } & 21\end{array}$

$\begin{array}{ll}\text { Immaterial } & 25\end{array}$

PART 3: MATERIAL ARCHITECTURES 29

$\begin{array}{ll}\text { Granular Matter } & 30\end{array}$

State of the Art: Architectural granular material systems 32

$\begin{array}{ll}\text { Material Research } & 41\end{array}$ 
Particle Morphology $\quad 44$

$\begin{array}{ll}\text { Aggregation Processes, Methods and Application } & 78\end{array}$

Architectural Material $\quad 92$

$\begin{array}{ll}\text { Projects } & 93\end{array}$

Design Exploration 1: Sands of Tomorrow — Adaptive Architecture Studio to Sukkah 94

Design Exploration 2: Flow (Winter Stations 2016) 100

Design Exploration 3: Seeds (Designing for Urban Agriculture - Allan Gardens) 124

Materials and Methods 133

PART 4: IMMATERIAL ARCHITECTURES 135

The Creative User 136

Design Exploration 4: JaXX 149

$\begin{array}{lr}\text { PART 5: REFLECTION } & 159\end{array}$

$\begin{array}{ll}\text { Research } & 160\end{array}$

$\begin{array}{ll}\text { Design } & 161\end{array}$

PART 6: UNCERTAIN FUTURES 163

Sites of Chaos - Natural Disaster Relief 164

$\begin{array}{ll}\text { Defending Freedoms } & 168\end{array}$

$\begin{array}{ll}\text { Malleable Matter } & 170\end{array}$

$\begin{array}{ll}\text { Creative Chaos } & 172\end{array}$

$\begin{array}{lr}\text { APPENDICES } & 177\end{array}$

$\begin{array}{ll}\text { BIBLIOGRAPHY } & 216\end{array}$ 



\section{LIST OF TABLES}

$\begin{array}{lr}\text { Table 1. Effect of the base condition on pile stability } & 48\end{array}$

Table 2. Effect of vibration on pile stability for columns of $d=8$ ” and $\mathrm{h}=50 \mathrm{~cm}$ for base particle

Table 3. Effect of arm length on pile stability

Table 4. Effect of mixed scaled particles on pile stability

Table 5. Effect of arm quantity on porosity and pile stability

Table 6. Effect of material (friction) on pile stability 


\section{LIST OF FIGURES}

Images and illustrations by author unless otherwise noted.

Figure 1. Villa Spatiale. From ArchDaily. Retrieved from http://images.adsttc.com/media/images/ 56a8/a5ca/e58e/cee7/e100/003a/slideshow/IMG_1065.jpg?1453893054. @ Yona Friedman

Figure 2. View of New Babylonian Sectors. From Gemeentemuseum Den Haag, [Photo] by Tom Haartsen, Retrieved from http://www.gemeentemuseum.nl/en/organisation/news/150-worksby-dutch-artist-constant-off-to-madrid (C) Constant / Fondation Constant c/o Pictoright Amsterdam (2016)

Figure 3. Fun Palace interior perspective. Adapted from CCA, by C. Price, ca.

1960-1964, Retrieved from http://www.cca.qc.ca/img-collection/iJYnk-JL_ ChdZ9mp35cLhpjnbhk=/1400x936/378817.jpg. () CCA

Figure 4. Cushicle + Suitaloon in three configurations. From The Archigram Archival Project, by M. Webb, n.d., Retrieved from http://archigram.westminster.ac.uk/project.php?id=92

Figure 5. Primary steel structure for the 'Bird's Nest'. From ArchitectureWeek, by Arup, n.d., Retrieved from http://www.architectureweek.com/cgi-bin/awimage?dir=2008/ 0827\&article=building_1-2.html\&image=13981_image_6.jpg

Figure 6. Walt Disney Concert Hall. 2005, Retrieved from https://commons.wikimedia.org/wiki/ File:Image-Disney_Concert_Hall_by_Carol_Highsmith_edit.jpg

Figure 7. Tumbling Units tectonics. Reprinted from “Tumbling Units," by K. Tsubaki, 2012, Matter: Material processes in Architectural Production, 196.

Figure 8. Tumbling Units canopy detail. Reprinted from "Tumbling Units," by K. Tsubaki, 2012, Matter: Material Processes in Architectural Production, 199.

Figure 9. Hygroscopic particle can curl in response to humidity. From M Helmreich, 2015, Retrieved from http://www.karoladierichs.net/wp-content/gallery/tea_mhelmreich/tea_ mhelmreich_02.jpg

Figure 10. Behavior-based Robotic Strategies for Adaptive Design of 
Aggregate Systems. From O Kyjanek, L Yunis, 2015, Retrieved from

http://www.karoladierichs.net/wp-content/gallery/kyjanekyunis/tea_kyjanekyunis_01.jpg 37

Figure 11. Gripping effector tests with cable robot for Aggregate Pavilion 2015, Retrieved from http://icd.uni-stuttgart.de/?p=14631. (C) ICD University of Stuttgart 2015

Figure 12. Aggregate Pavilion 2015, Retrieved from http://icd.uni-stuttgart.de/?p=14631.

(C) ICD University of Stuttgart 2015

Figure 13. Close up of Rock Print. From Gramazio Kohler Research, 2015, Retrieved from http://gramaziokohler.arch.ethz.ch/web/e/forschung/297.html @ Gramazio Kohler Research, ETH Zurich

Figure 14. Sequence of research experiments

Figure 15. The common design principle of the particles tested consist of two identical plates with slot joints

Figure 16. Designed granular display unique aggregation properties. Base particle shown

Figure 17. Designed granular materials are able assume columnar forms with a vertical angle of repose

Figure 18. Selection of various particle morphologies

Figure 19. Base condition with corrugated cardboard mat

Figure 20. Vibrated column

Figure 21. Non-vibrated column

Figure 22. 10" d column of $100 \mathrm{pc}$, base particles

Figure 23. 5" d column of $100 \mathrm{pc}$, half-scaled particles

Figure 24. Attempt to form 5" d column of $100 \mathrm{pc}$, truncated particles

Figure 25.5 " d column of $28 \mathrm{pc}$, medium-length truncated particles

Figure 26. 200 pc equal mixture of base and half-scaled particles

Figure 27. 200 pc equal mixture of base and truncated particles

Figure 28. 70 pc base particle 
Figure 31. a) 8-arm column (74 pc) supporting 4 pounds; b) 12-arm column (50 pc) supporting 3 pounds

Figure 32. Pre-straining increase rigidity

Figure 33. The aspect ratio of the particle is controlled by the angle between arms

Figure 34. Stratified mixture of particles

Figure 35. Close up of layers (top to bottom): 4-arm (140pc), 8-arm (35 pc), 12-arm (35 pc)

Figure 36.74 pc, 8” d columns; acrylic particles

Figure 37. 74 pc; rubber coated particles

Figure 38. Rubber coated particle held together by material friction

Figure 39. Form with greater projecting elements enabled by greater material friction

Figure 40. Greater formal possibilities with rubber particles

Figure 41. Mixture of acrylic and rubber particles

Figure 42. Different arm articulations: (a) "bulb", (b) "serrated", (c) "hook"

Figure 43.74 pc hook particle

Figure 44. Testing cohesion of various particles

Figure 45. Hanging formation made possible by hooks

Figure 46. Hook particles able to withstand tensional forces is capable of forming large cantilevers

Figure 47. Mixture of rubber and hook particles attempting a cantilevered arch

Figure 48. Expressive formal possibilities can be afforded with the right mix

Figure 49. Vault enclosure

Figure 50.Continuous feedback and adjustment is required to achieve desired forms as the processes of design and building are blurred 
Figure 51. Close up of hybrid ordered and self-organized enclosure

Figure 52. Inflatable formwork

Figure 53. Array of particles linked with string

Figure 54: Top particle is held up fully by the tension of the string

Figure 55. String binding post aggregation

Figure 56. Diagram of granular vacuum jamming. By A.Mozeika, 2015, Retrieved from http://www.annan.io/articles/54

Figure 57. Hybridized system utilizing strengths of both systems

Figure 58. Particles jammed into a solid state

Figure 59. Large components assembled outdoors

Figure 60. Detail of large components

Figure 61. Physics rigid body simulation using Blender software

Figure 62. Construction concept. By author and Victor Huynh

Figure 63. Spatial qualities from interior

Figure 64. Sands of Tomorrow Sukkah proposal. By author and Victor Huynh

Figure 65. Flow Winter Station proposal. By author and Victor Huynh

Figure 66. Layout of 4'x8' sheet material for CNC milling. 27.8\% efficiency (left) 32.5\% efficiency (right)

Figure 67. Flow test build in the fabricator's shop

Figure 68. Dowel pin connection

Figure 69. Shock cord connection

Figure 70. Location of Flow at Toronto's Woodbine Beach

Figure 71. Installation under construction. By Johnson Tang, 2016

Figure 72 . The beach setting was with sand and uneven terrain was desirable 
Figure 73. Flow newly constructed

Figure 74 . The rope binding system became too cumbersome to get around the form

Figure 75. Particles naturally submerged into the sand provides a stable foundation

Figure 76. Snow accumulation on structure

Figure 77. Close up view of snow accumulation from "inside"

Figure 78. Ice buildup can bind particles together

Figure 79. Flow filtering sunlight

Figure 80. Component close up showing wear

Figure 81. Lighting effects from interior in low light. By Johnson Tang, 2016

Figure 82. Visualization of screen proposed for Allan Gardens

Figure 83. Visualization of pyramid trellises proposed for Allan Gardens

Figure 84. Transport of components

Figure 85. Overall constructed screen with seating

Figure 86. Wildlife engaging structure

Figure 87. Seed as armature for plant growth

Figure 88. Fab Tree Hab Concept. From Terrefrom ONE, n.d. Retrieved from http://www.terreform.org/fab_tree_town_sml3.jpg

Figure 89. Visitors discovered that the form can be modified

Figure 90. Some visitors unsure whether to engage

Figure 91. The form would continue to evolve

Figure 92. Public record of transformation gathered from Instagram photos

Figure 93. Form can be carefully sculpted

Figure 94. Through participation, the form remained mostly as a pile 
Figure 97. Bloom. From Flickr, by BloomGame, 2012, Retrieved from https://www.flickr.com/photos/83738914@N03/7666983198/

Figure 98. Examples of Spicule appropriation. By Léopold Lambert, 2008, Retrieved from http://www.leopoldlambert.net/2014/12/08/spiculation-2008/

Figure 99. JaXX employed in various applications

Figure 100. Diagram of modular system concept

Figure 101. Its generic module is exploited to stimulate creativity and play as a constructional toy concept.

Figure 102. Tsumiki toy. Designboom, 2015, Retrieved from http://www.designboom.com/ architecture/kengo-kuma-tsumiki-pavilion-tokyo-design-week-triangle-shaped-wooden -blocks-12-09-2015/ (C) Ikunori Yamamoto

Figure 103. Tsumiki as installation. Designboom, 2015, Retrieved from http://www.designboom.com/architecture/kengo-kuma-tsumiki-pavilion-tokyo-design-week-triangle-shaped-wooden-blocks-12-09-2015/ @ Ikunori Yamamoto

Figure 104. Chidori toys. Detail, 2011, Retrieved from http://www.detail-online.com/blog-article/ chidori-furniture-by-kengo-kuma-25600/

Figure 105. Chidori furniture. Detail, 2011, Retrieved from http://www.detail-online.com/ blog-article/chidori-furniture-by-kengo-kuma-25600/

Figure 106. GC Prostho Museum Research Center. ArchDaily, 2012, Retrieved from http://www.archdaily.com/199442/gc-prostho-museum-research-center-kengo-kumaassociates/5004e0bb28ba0d4e8d000ad0-gc-prostho-museum-research-center-kengokuma-associates-photo (C) Daici Ano

Figure 107. Visualization of material deployed as shelter in disaster region. ChiefHira (https://commons.wikimedia.org/wiki/File:Damage_of_Tsunami_at_Onagawa_01.JPG), Damage of Tsunami at Onagawa 01“, Collage by Calvin Fung, https://creativecommons.org/licenses/by-sa/3.0/legalcode 
Figure 108. The system is valued for its rapid deployment and ease of construction. ChiefHira (https://commons.wikimedia.org/wiki/File:Tōna_Station_after_Tsunami.JPG), Tōna Station after Tsunami“, Collage by Calvin Fung, https://creativecommons.org/licenses/ by-sa/3.0/legalcode

Figure 109.The material is able to adapt to the unknown and harsh conditions. ChiefHira (https://commons.wikimedia.org/wiki/File:Shizugawa_Station_platform_after_ Tsunami.JPG), Shizugawa Station platform after Tsunami“, Collage by Calvin Fung, https://creativecommons.org/licenses/by-sa/3.0/legalcode

Figure 110. Czech Hedgehogs, Retrieved from https://commons.wikimedia.org/wiki/File:

Rozsochac.JPG

Figure 111. Sketch of capsule concept

Figure 112. Conceptual visualization matter consisting of designed micro-aggregate substructure

Figure 113. SandboXX interactive environment 


\section{LIST OF APPENDICES}

Appendix A: Fluid Architecture: Pneumatically informed aggregate system of designed particles

Appendix C: Flow (Winter Stations 2016)

Appendix D: Seeds (Allan Gardens)

Appendix E: JaXX (Mako Student Innovation Award Finalist)

Appendix F: Speculations and Explorations 



\section{INTRODUCTION}

"change is the only permanence, and uncertainty the only certainty." 


\section{Context}

The success of human civilization relies on the capacity for adaptability in the face of change. As nomadic creatures our movements were once intimately connected to the seasons and bound to the patterns of animal and land systems. With time, in response to changes in climate, the surface of the earth, and other living forms, we experienced a shift from hunter-gathering to sedentary societies. Additionally, with the development of these societies, Manuel DeLanda, summarizing physicist Arthur Iberall's account in terms of flows and phase transitions, describes it as "fluidlike" social formations 'crystalized' into stratified civilizations" (1992, p. 154). DeLanda contends that early societies may have achieved a "better consistency among their flows, a viscosity more in tune with their ecosystem than our own” (1992, pp. 154-155).

Today, technological, social, and ecological shifts across the world are dramatically affecting our lives. Customization and greater access to information has brought upon us great variety and choice. Our current contemporary condition is characterized by constant and rapid change. It is what sociologist Zygmunt Bauman defines as a "liquid" modernity. Consequently, within this free flowing state, a cause for concern for Bauman is the societal shift emphasizing the individual. As social structures struggle to keep pace with relentless change, the individual is left responsible for managing their own life leading to uncertainty and anxiety (2012). I argue, for the contrary, for the individual, but empowered and an embrace for the productive potential of uncertainty through the very concept of 'fluidity'. Fluid times calls not for fixed solutions, but flexible responses. Like DeLanda states, we should be focusing on "destratifying hardened institutions, setting into flux human practices that have sedimented" (1992, pp. 154-155). This could be extended to the practices of architecture and the forms of our shelters that sustain us.

From its processes to its products, architecture is a large and notoriously slow enterprise. It is within this context that architecture must shake its image of being inert and immutable. The values of permanence and solidity maintained from the past no longer reflect the dynamic and diverse aspirations of contemporary complex societies and the indeterminate situations of the natural environment. It has fallen out of sync with our ways of life defined by the instantaneous, unpredictable, and fleeting. We instead, are entrapped in crystalized constructs allowing set plans to dictate our actions and are constrained to adapt to our architecture. Little thought is given to how much of our lives and routine are governed by our built environment as we accept our buildings to be a constant, immoveable, and requiring too much effort to change.

The activities of people and the built environment are interconnected and inseparable. As life evolves, we need an architecture that is expressive and reflective of the structure and principles of current 
society. Perhaps the way to adapt is to consider allowing the people to have an active role in shaping their own spaces. For the one that understands individual desires and needs best should certainly be the individual. People introduces freedom and creativity, and with that chance acquires a great role in every spatial situation and experience. The complexity of the world necessitates a more complex architecture to respond.

The term architecture as a general term refers to the organization and internal infrastructure of a natural or culturally produced object (Weinstock, 2010, p. 8), and usually considered to be complex and carefully designed. Within the context of the discipline of architecture, this definition is relatable and can be closely associated with its activities. Its forms are the organization of matter, people, and ideas, which makes the architect an organizer. Michael Weinstock in The Architecture of Emergence asserts that, "all forms of the world have 'architecture" and, "to study form is to study change" (2010, p. 9). The forms of nature and the forms of civilization have emerged, evolved, flourished, and collapsed to have new forms emerge from their reorganization (Weinstock, 2010, p. 9). To begin to explore change in architecture is to consider change in its organization, in terms of material and immaterial terms, and also a recognition that with change comes uncertainty.

A reconceptualization of architecture is necessary, one not focused on the certainty of solutions or forms, but one patterned by dynamic feedback of human and environmental forces. It requires system-based thinking and accepting uncertainty to combat uncertainty. Appropriating observed phenomena from natural systems, this is found in the logics of granular matter.

\section{The Form of the Thesis}

Through a series of experiments and design explorations it is revealed how granular material can become a useful model for thinking, creating, and practicing architecture. In the spirit of embracing the uncertain, this thesis emerged and evolved through discoveries and conclusions made upon reflection while observing granular material constructions perform in a real setting. At times, more questions are raised than answers given which then prompts further dialogue. Discussions of the design explorations at times are enhanced with case studies that demonstrate similar applications or approaches.

Part 1 begins with a background on the fixation with permanence and stability revered by architecture. Next, the notion of adaptability as a response is examined through tracing its lineage. Embracing systems thinking and uncertainty is discussed as a productive approach in Part 2 with its implications for the material and immaterial systems of architecture. These concepts are found to be manifested in complex systems in nature. Part 3 introduces granular matter into the material space of architecture through a series of empirical investigations and applications. Its novel physical and procedural capacities 
are explored and discussed with considerations of form, structure, performance, and transformation. Emerging from the observations and outcomes of the design explorations, Part 4 reveals important considerations for practice in terms of the relationship of architect, user, design and agency. Part 5 provides a reflection on the merits and impacts of a designed granular architecture. Finally, Part 6 concludes the thesis by provoking thought on the emerging field, speculating on possible futures for granular material and architecture. 


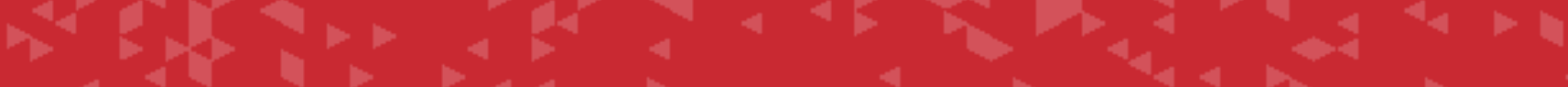
Notis 8

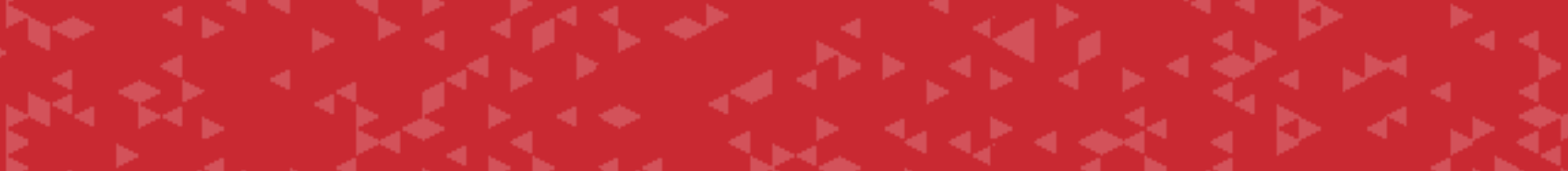

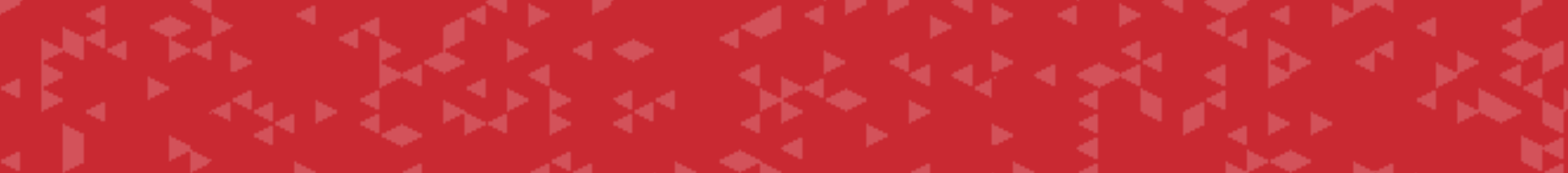

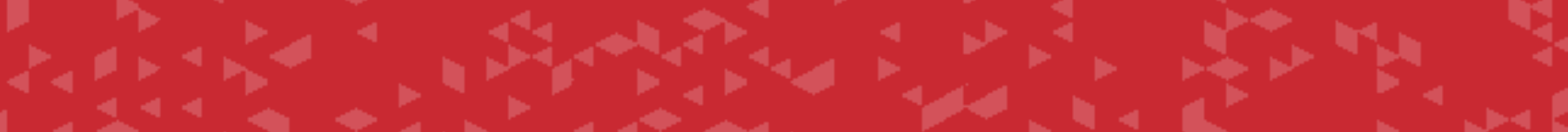

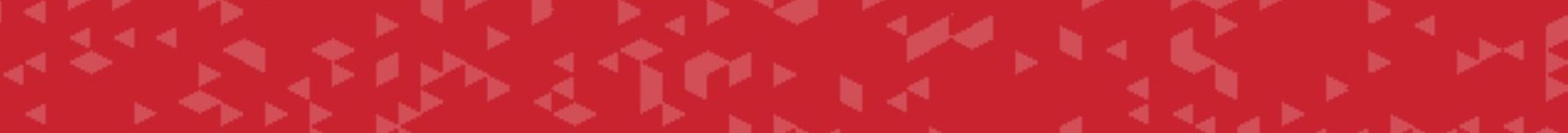
a

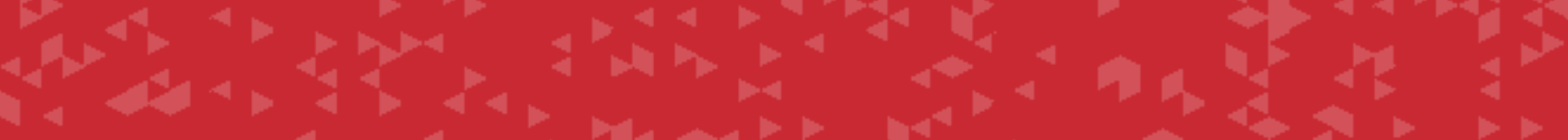

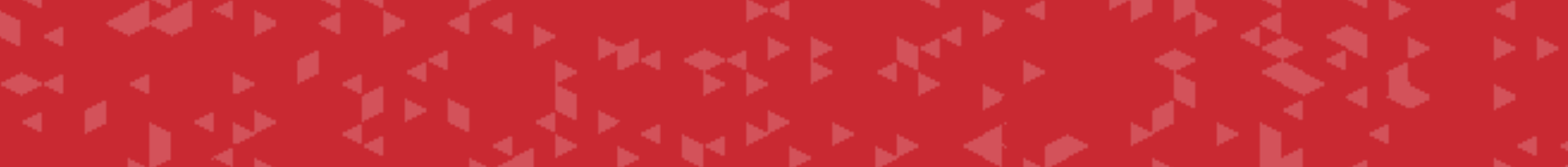
स 4 a

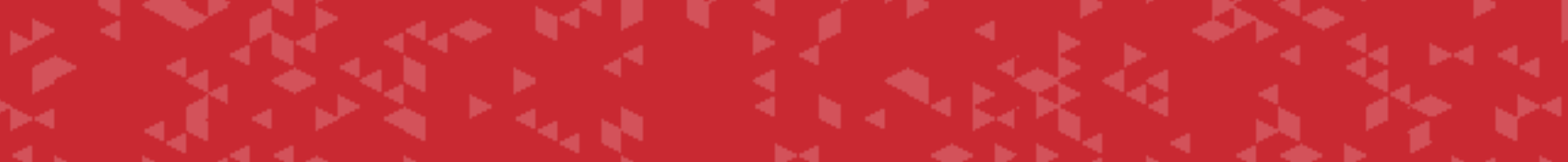

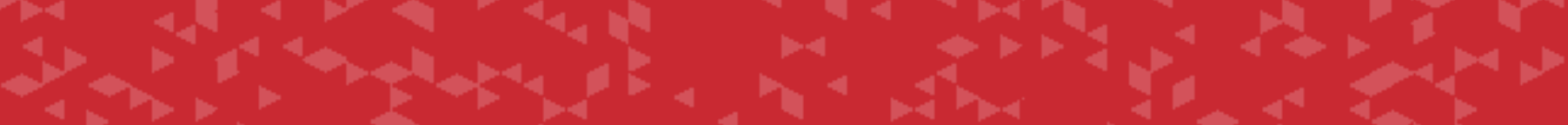

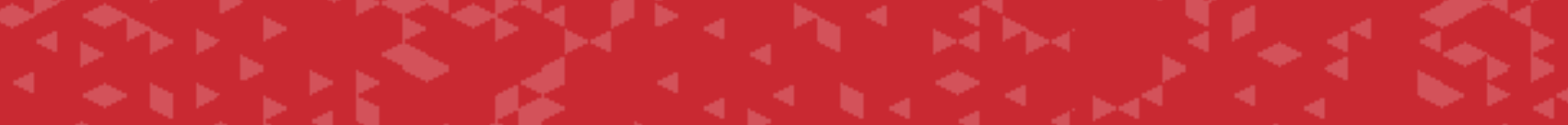
art axy ins

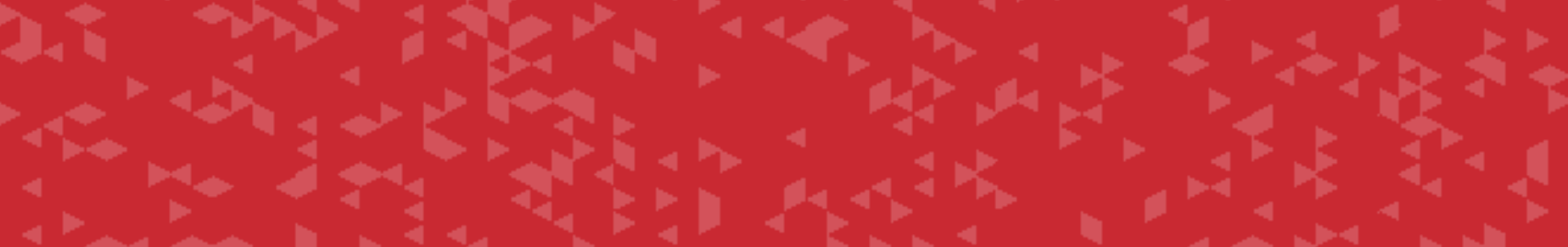

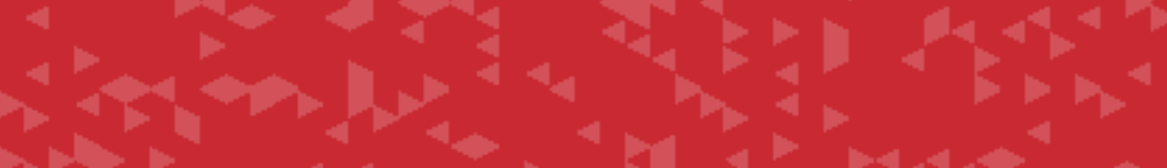

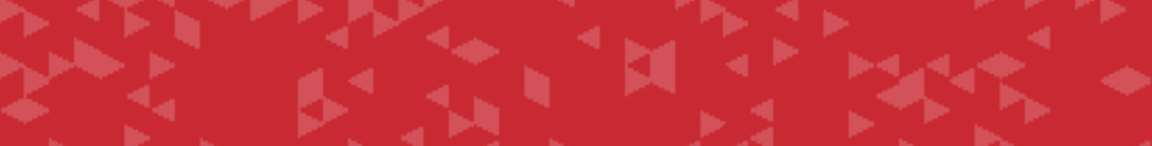
a tis

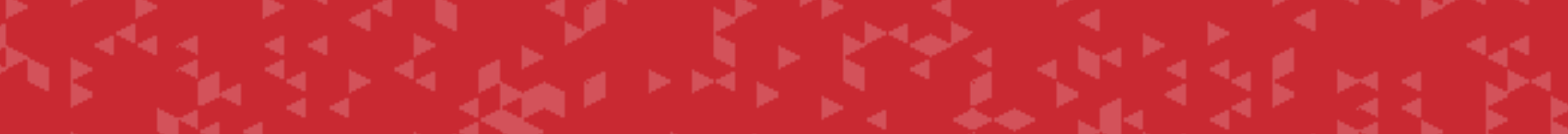
x 4.

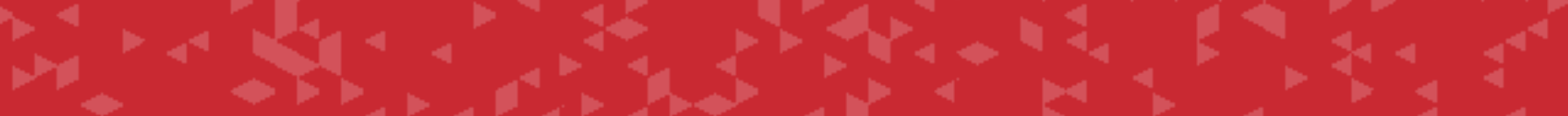
- 


\section{PART 1: BACKGROUND}

"We are accustomed to thinking of [architecture] exclusively in terms of physical, mute, immutable objects; architects themselves like to photograph their buildings unfinished, silent and empty. It could be said that architecture is concerned solely with material forms, cold and intangible, situated beyond time."

- Luis Fernández-Galiano

Fire and Memory (2000) 


\section{Firmitas}

Firmitas, utilitas, venustas, with those principles Vitruvius set the foundation of Western architecture. Firmitas (firmness) determines strength and solidity in its material condition and expresses, stability and permanence-its capacity stand against the passage of time. This physical consistency relates to its formal solutions that allow structures to stand, but a "firm, stable architecture is also a solid architecture," Ignasi de Solà-Morales notes, "whose dimensional and formal characteristics do not change in spite of environmental fluxes" (1998, p. 36). This permanence is achieved through delimiting space with solid materials as Morales claims, "the material condition-physical consistency, solid construction, delimitation of space-has for more than 25 centuries linked the knowledge and technique of architecture to permanence" (1998, p. 36). Morales questions:

Is there an architecture that is materially liquid, that configures and is attentive not to stability but to change and is thus at one with the fluid and shifting nature of all reality? Is it possible to think an architecture that is more of time than of space? An architecture whose objective would be not the ordering of dimensional extension but of movement and duration? (1998, p. 36)

In the dynamic contemporary climate of change and transformation, these are pertinent and increasingly urgent questions. Solidity and its crystallization of space denies openness and multiplicity. It makes the environment much less responsive and resilient. To strive for permanence is to battle against process and time.

Jeremy Till provides further insights on architecture's relationship with time. "Time," Till claims, "is engaged with as an enemy of architecture," because it is time's complexity that brings uncertainty to architecture. It is change, or flux, as Till puts it, that is one of the most dangerous aspects of time and thus has been something that architecture has commonly, and traditionally resisted (Till, 2009). Yeoryia Manolopoulou echoes this, but identifies the key sources of flux when she discusses the concept of chance in architecture: "To talk about chance is to talk about time, the simultaneity of different human activities and the fugitive intervention of the environment, all intertwined with the relative permanence of the building." (2013, p. xvii)

Temporality introduces not only forces of use and change into architecture, but also entropy. Entropy is problematic to architects as it denotes a condition of ongoing uncertainty and with it the potential of decline into disorder (Till, 2009). Entropy however, can be harnessed by architecture; it implies an ongoing process of building the unfinished, as change is ever present. In his book Architecture Depends, Till posits that architecture is shaped more by external forces than internal processes of the architect 
and that the dependency on these external contingencies are frequently denied which in turn poses a great problem to architecture (2009, p. 1). That problem is the conflict between, what architecture actually is - dependent and contingent - and what architects insist it to be-autonomous and controlled/ planned. Till notes, through the work of Indra McEwen, that the conflict is traced back to Vitruvius. Through the dominant metaphor of the classical body in his writings, Vitruvius identifies architecture as "an act of imposing order, of taking the unruly and making it coherent" (Till, 2009, p. 28). As such, the general thrust in architecture has mostly been to rid the world of contingency to better manipulate that world into (a semblance of ) order (Till, 2009, p. 37). 


\section{Lineage}

It is important to trace the development of adaptable architecture to better situate this thesis within the discourse. Adaptable, dynamic, interactive, responsive, transformable - are among the various terms used to describe changing environments. In modern Western architectural discourse, the desire for spaces and objects to meet the dynamic needs of the evolving individual, society, and environment peaked in the 1960s. This corresponded to a period of upheaval characterized by environmental awareness, social transformation and technological innovation. During this time many conceptual architectural projects formed from thinkers such as Yona Friedman with his Ville Spatiale utopia (Figure 1), Constant Nieuwenhuys' (Constant) New Babylon megastructure, and Archigram's nomadic and pneumatic structures. While the projects were often political and critical in nature, it nonetheless advanced ideas of a different type of living. They were focused on individual expression-personal fulfillment through autonomy and freedom to control their own lives right down to the built environment-not unlike the social climate of today. Common characteristics of the projects were that they often focused on the user, have no specified program, forms were uncertain, and they were participatory. The following is a look at the different paradigms of adaptability from the urban scale to the personal.

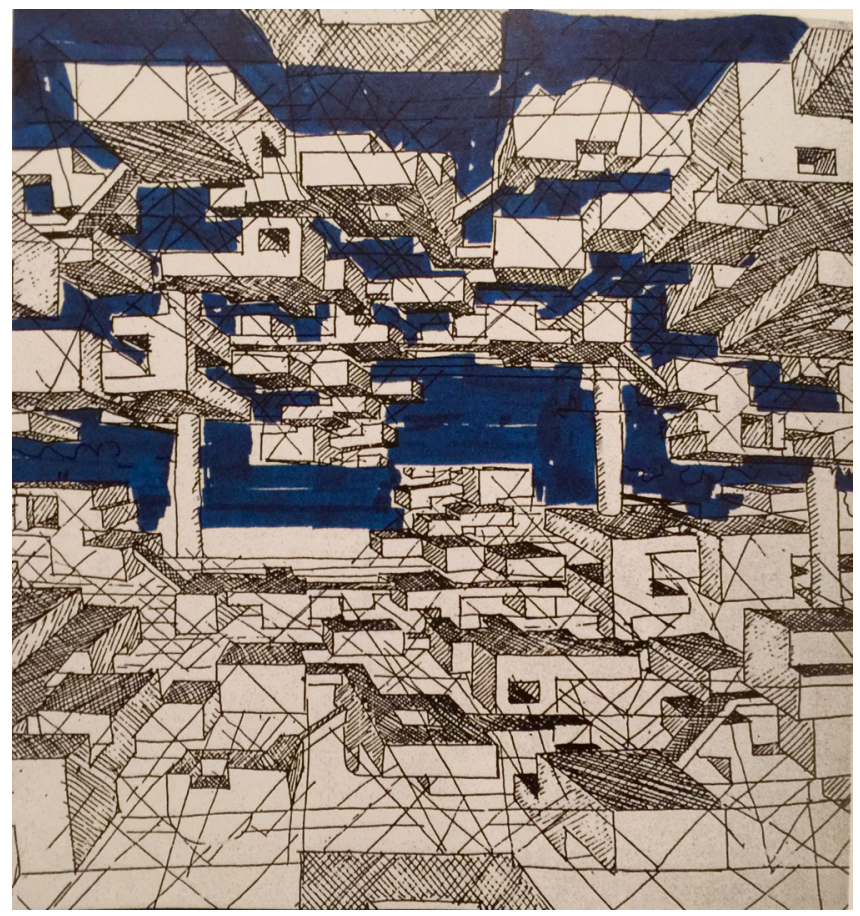

Figure 1. Villa Spatiale 


\section{Situationism}

Established in 1957 and dissolved in 1972, the Situationist International (SI) opposed both functionalism and the architecture profession. Functionalism was rejected because it was thought to respond only to abstract needs and promoting non-participation instead of genuinely considering the real desires of individuals (Heynen, 1999, p. 157) Existing architecture and urban structures were seen as products that contributed to the oppression of society by imposing its norms and values, conditioning the individual to conform, and thus suppressing creativity and autonomy (Heynen, 1999, p. 173). Simon Sadler writes: "The benign professionalism of architecture and design had, in their opinion led to the sterilization of the world that threatened to wipe out any sense of spontaneity or playfulness" (1999, p. 5).

Creativity, which was thought to be ultimate freedom, can be achieved through several situationist practices. One practice, the dérive, is a playful-constructive behaviour of drifting through the city and appropriating existing spaces. The central idea however is the construction of situations - the deliberate distortion of a certain matter or space in order to subvert the social conditioning. According to Guy Debord, a leader of the SI movement, "architecture must advance by taking emotionally moving situations, rather than emotionally moving forms, as the material it works with. And the experiments conducted with this material will lead to new, as yet unknown forms." The strategies implies a dynamic environment in contrast to the solidity and immobility of traditional buildings. An emphasis is given to the user or hybrid producer-user as the creator of environments rather than the single author.

Situationist strategies are of considerable value to the notion of agency because of the significance it gives to the creative user. However, the Situationists' distrust of form limits their relevance to architects. Adrian Forty describes this distrust as "an opposition to the process of reification, of the tendency of capitalist culture to turn ideas and relationships into things whose fixity obscures reality" (2000, p. 170). As such, Sadler notes: "most of the architecture and spaces that were endorsed by Situationists "existed by chance rather than by design: back streets, urban fabric layered over time, ghettos" (1999, p. 159). This tension was evident with the eventual departure of Constant, a prominent figure of the SI, who conceptualized a situationist city. 


\section{Case Study: New Babylon - Constant, 1958}

Constant produced an extensive study consisting of sketches, painting, and models. His project New Babylon was envisioned as a layered megastructure suspended above existing cities that was composed of a society free to playfully drift through having been liberated from all "useful" production needed for subsistence enabled by new automation technology (Constant, 1974). Conceived as a dynamic labyrinth, New Babylon could be continuously restructured by the spontaneity and creativity of its inhabitants. "It is a chaotic terrain with mobile building elements and environmental control devices allowing people to actively construct moods and develop new forms of behavior" (Wigley, 1998, p. 13). New situations would arise from new forms and new forms would be created by new situations suggesting a possibility of endless experimentations and constant new encounters where disorientation and confusion are all valued experiences that further adventure and play. Constant had taken to call the inhabitants Homo Ludens (Man the Player) and declared that "the real designers of New Babylon will be the Babylonians themselves" (1974). Hill states, "in Situationism, as defined by its original members, one of the traditional roles of the architect, the design of form expected to last for a number of years, is absent and even irrelevant; the only role for the architect is as the creator of objects for appropriation" (2003, p. 70).

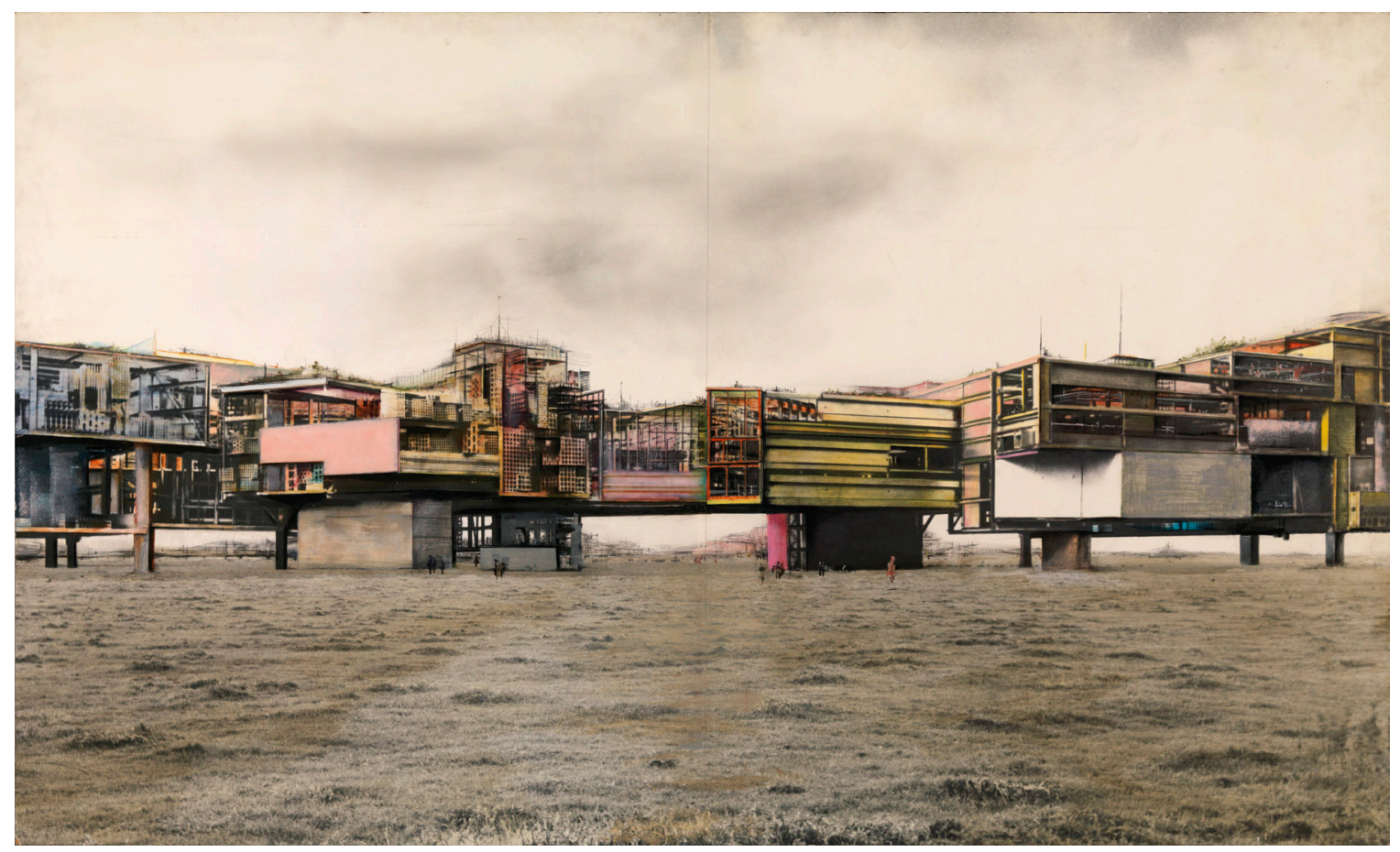

Figure 2. View of New Babylonian Sectors. 


\section{Cybernetics}

Constant closely followed the arguments of Norbert Wiener who is considered the originator of

cybernetics theory. Its relevance to architecture was introduced by Gordon Pask. Cybernetics is systems oriented thinking: a study of the ways in which complex biological, social or mechanical systems organize themselves, regulate themselves, reproduce themselves, evolve, and learn (Pask, 1968). Pask saw architecture as a conversational system: "it perpetually interacts with its inhabitants, on the one hand serving them and on the other hand controlling their behaviour" (Pask, 1969, p. 494). In this sense, the designer of such architecture has an immense responsibility, for each decision results in a physical development that greatly influences our lives.-The computer and electronics were given a large role in facilitating this feedback, monitoring people's desires and acting on them. This is most notable in the Fun Palace_-an unrealized project by Cedric Price.

\section{Case Study: The Fun Palace - Cedric Price, 1961}

The architecture of the Fun Palace, the seminal work by visionary architect Cedric Price, would be derived according to principles from the diverse fields of uncertainty theory (from physics), cybernetics and Situationism (Manolopoulou, 2013). Unlike Constant and Archigram's visions, the Fun Palace was commissioned as a real project that was carefully designed and very nearly built. A conventional architectural project however, it was not: with an unspecified program and uncertain form, it was antithetical to normative architectural practice which Price thought was overdetermined (Mathews, 2005). Consisting of a framework of gantry cranes and prefabricated modules, almost everything was moveable. It was a socially interactive machine-constantly changing, indeterminate, and highly adaptable to the educational and entertainment desires of its users (Figure 3). Price stated that it was "a kit of parts, not a building. I doubt whether it will ever look the same twice" (Price as cited in Mathews, 2005, p. 80). This was because architecture for him, was an event always in the process of becoming, unfolding in time rather than a formal object. He thus approached problems in terms of performativity. "The Fun Palace would then simply be an entity whose essence was events in continual flux, which adapted itself spatially to accommodate multiple and indeterminate uses" (Mathews, 2006).

Price's work had strong social motives, however it would be its mechanical and technological formal imagery that would become greatly influential towards a mechanistic, multifunctional/flexible paradigm for adaptability. As Manolopoulou writes, Price's pioneering work "should be seen within the broader interdisciplinary spectrum of the arts and politics of the time" bringing together very different areas of knowledge into a "distinct design mode that attempted to link technology, space [...] and society" (2013, p. 210). His concept of Anticipatory Architecture accepts uncertainty as a positive opportunity through all stages of production, from conception and design to construction and inhabitation (Manolopoulou, 2013). 
Pask would eventually be recruited to lead the project's Cybernetics Subcommittee. They were charged with developing the control systems that would regulate the configurations and activities based on feedback and analysis of behavioural patterns. This would have enabled the Fun Palace to learn and anticipate future conditions and thereby reinforce a mutualistic relationship between user and architecture. The Fun Palace was to be a living, evolving organism and cybernetics a model of natural processes which would permit all living things to actively maintain the conditions of life in a changing world. Price would eventually get the opportunity in 1976, with the Inter-Action Centre to realize many of the social goals he had for the Fun Palace without the technical aspects (Mathews, 2007, p. 187).

The idea of architecture as a living, evolving process was carried forward by John Frazer, who was also a systems consultant on the Fun Palace. In An Evolutionary Architecture (1995), Frazer proposes nature as a model for generative forces in architecture, paralleling natural processes such as growth and evolution that respond to their changing environments. He draws from the wider context of natural science for biological and scientific analogies. Architectural concepts are expressed as generative rules and was tested virtually and physically through the many examples of work developed with his students. The goal was to "achieve in the built environment the symbiotic behaviour and metabolic balance that are characteristic of the natural environment" (Frazer, 1995).

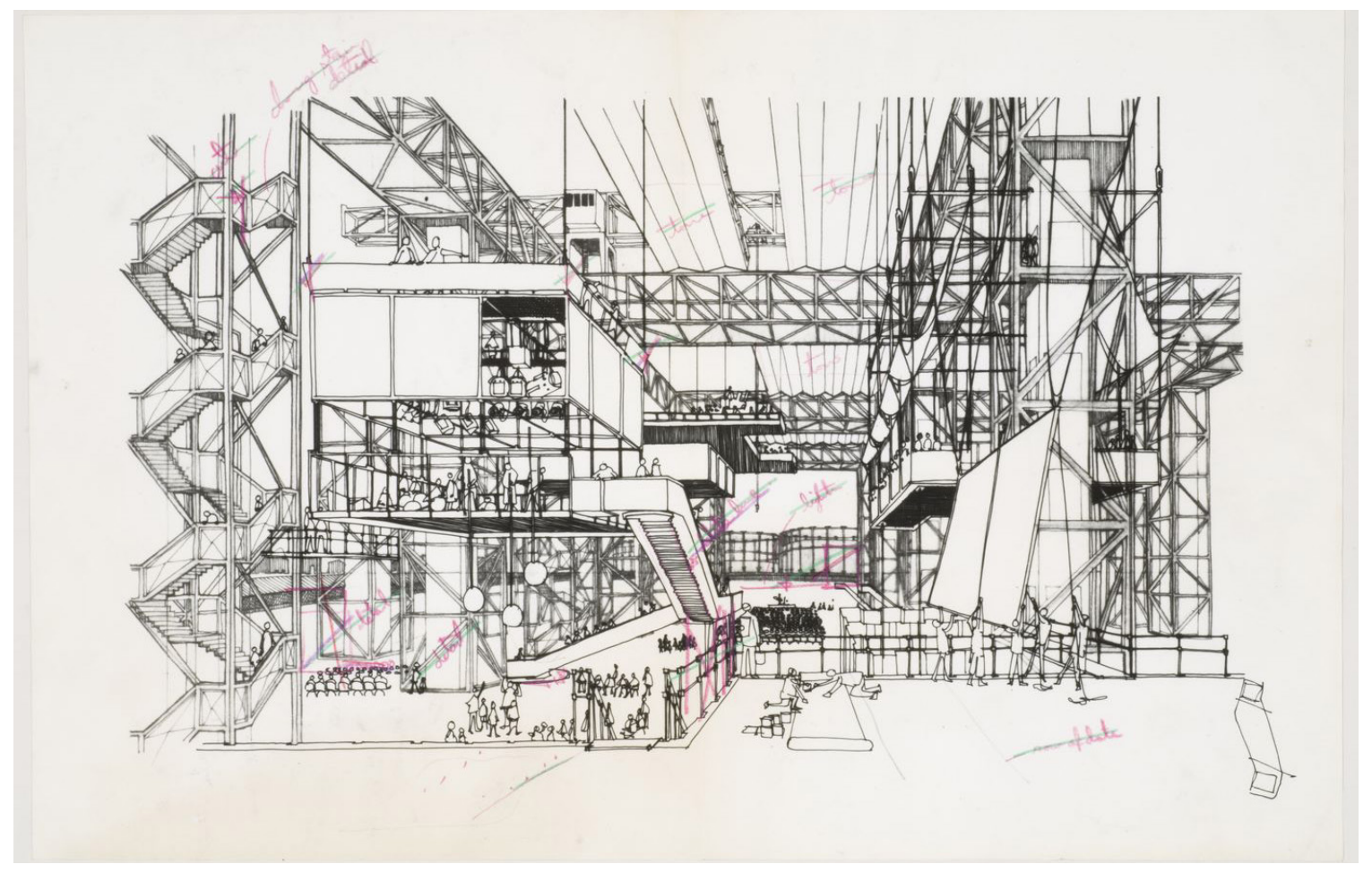

Figure 3. Fun Palace interior perspective. 


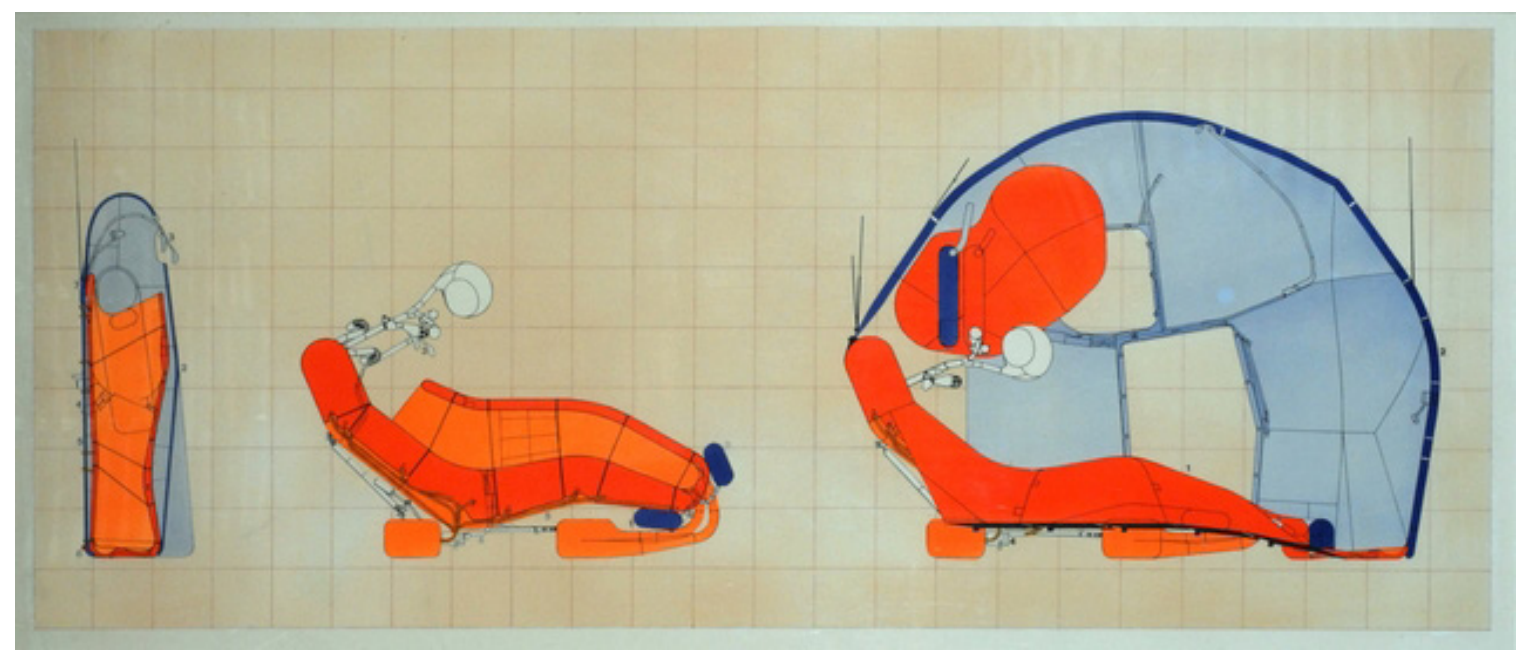

Figure 4. Cushicle + Suitaloon in three configurations.

\section{Soft Systems}

From the large megastructures and frameworks came a reaction refocusing efforts on individualistic counterculture via personal soft pods most prominently explored by the group Archigram. The imperative to them was to "create 'open ends', an architecture that expressed its inhabitants' supposed desire for continuous change" (Sadler, 2005, p. 94). Literal soft material constructions, such as inflatables, were important as it a democratic alternative, allowing for continuous change and portability (Steiner, 2005, p. 91). Examples of these include the Cushicle and Suitaloon (Figure 4).

In 1967 Nicholas Negroponte founded the Architecture Machine Group at MIT where he went on to formulate the concept of Soft Architecture Machines (1975). He described an architecture that was soft in terms of the type of materials, but more importantly soft in terms of the structures of architecturethe software/ processing of architecture. Intelligent, machine computing that would augment the large number of variables and contingencies required in the architectural process, would allow non-architects to participate in the design and realization of their homes. In promoting a computer aided participatory design process, Negroponte consequently seeks to create a physical environment with agency and diminish the authorship of the architect (1975, p. 1). Central to his idea of a responsive environment is user participation. Negroponte contends that the individual best knows their own needs and the best way to achieve user responsiveness is by providing simple tools and materials (in his case through the machine) that would empower the individual with competence to be their own architect and design their own spaces (Negroponte, 1975, pp. 99-100). He rejects alternative forms of participation such as 'advocacy' (Negroponte, 1975, p. 101) and the idea of the paternalistic or what Yona Friedman would call a "translator" architect and insists on giving the inhabitant the control (Negroponte, 1975, p. 102). 
To carry out the necessary physical transformation two classes of responsive materials were proposed: softs and cyclics. A soft material need not necessarily be physically soft such like foams, air and plastics, but what is important is its malleability - that is, "they exhibit motor reflexes through simple controls" (Negroponte, 1975, p. 147). For these types of soft materials "form is memory," upon which one can "push and pull” (Negroponte, 1975, p. 149). Clear examples of this type are inflatables. Cyclics operate at a larger time scale, implying an "ever-continuing building process" (Negroponte, 1975, p. 150); an ability to assemble and disassemble and reorganize as in natural systems. Critical to such systems is the aspect of reversibility. Implicit in this proposed system is its ability to actively adjust according to one's need as "design process and building process become one and the same, continually in operation" (Negroponte, 1975, p. 145).

While the 1960s and 70s' soft movement was seen to "enable individualism, responsiveness, nomadism, and anarchy", soft is seeing a resurgence in recent times, beyond the purely material sense-positioned as a "counterpoint to the permanent, static and hard systems that are no longer viewed as suitable to address contemporary urban complexities and their continual transformations" (Bhatia \& Sheppard, 2013b, p. 8). In contrast to Negroponte's focus on the individual and position on the architect, current thinking seeks to expand the role of the architect as well as to propose an architecture that engages its wider context. Soft then, "refers to the responsive, indeterminate, flexible, and immaterial systems that operate through feedback, organization and resilience. These complex systems transform through time to acknowledge shifting and indeterminate situations" (Bhatia \& Sheppard, 2013a, p. 1). 


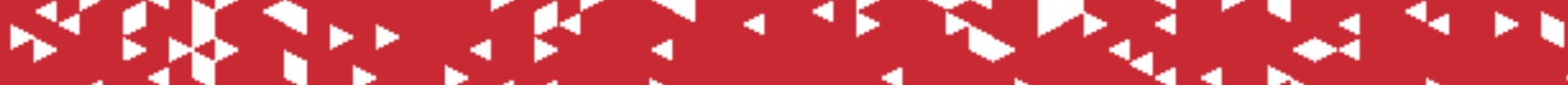

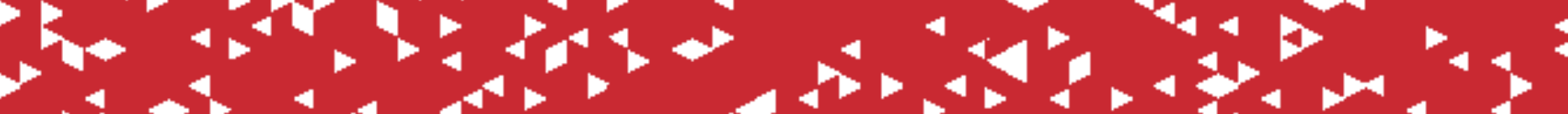
to 1. 40

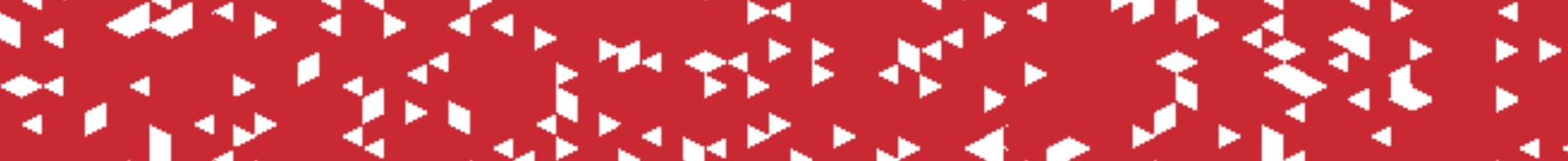
Q 4

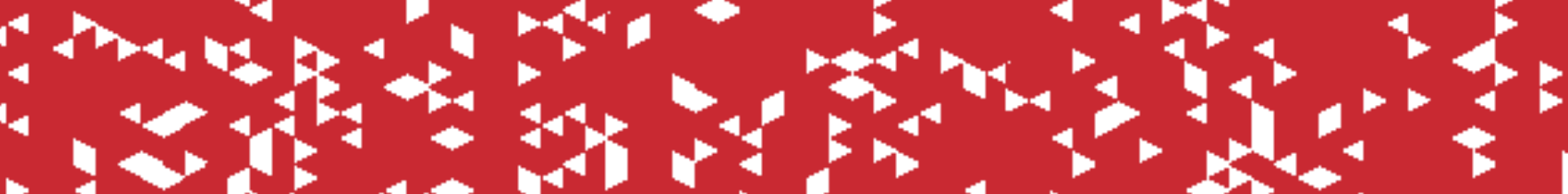

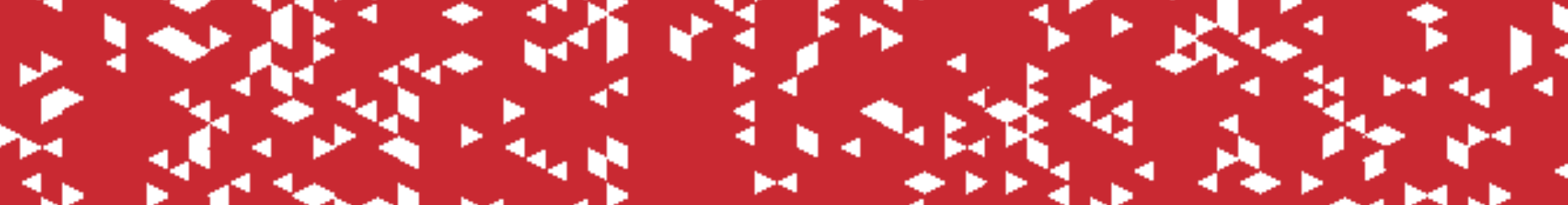

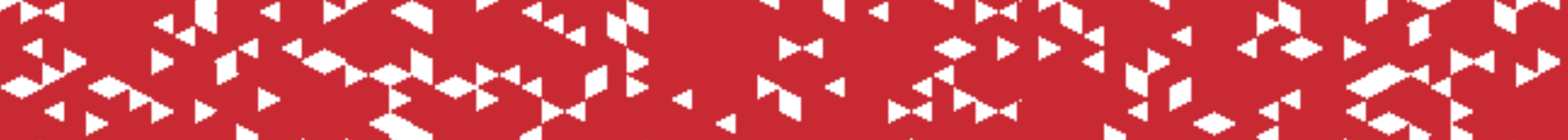
on

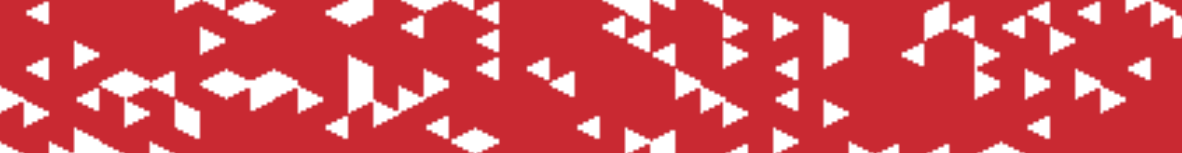

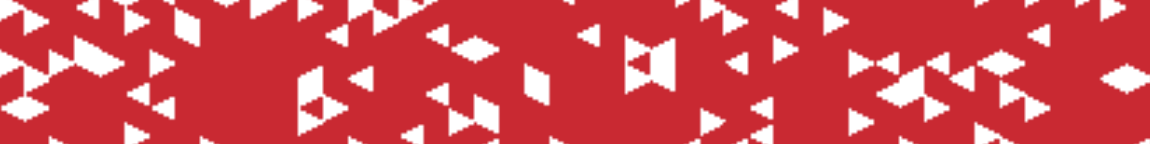
a tas sty 24

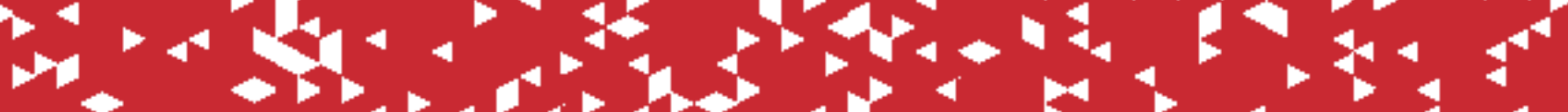

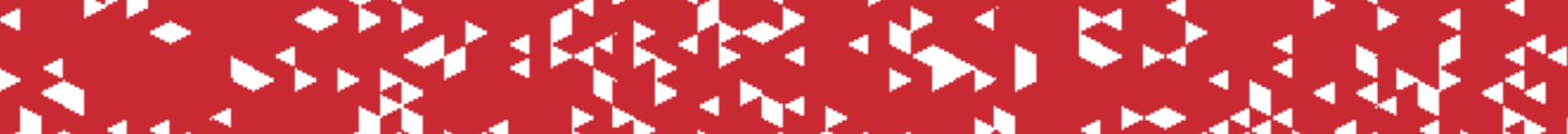

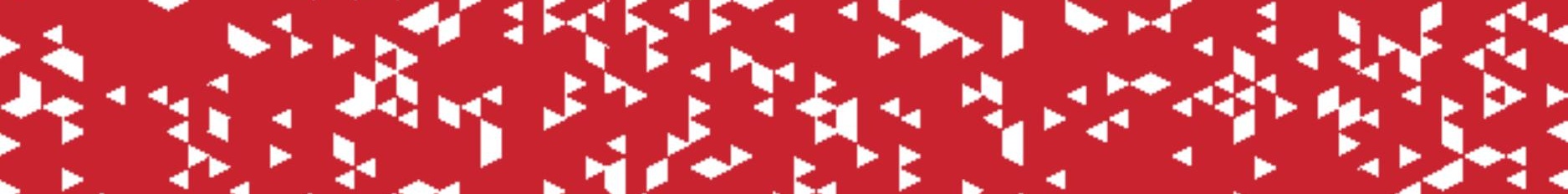




\section{PART 2: SYSTEMS APPROACH}

"[Earth's] system...is driven by its very "softness," its capacity to move, to differentiate internally, to absorb, transform, and exchange information with its surroundings, to develop complex interdependent sub- and super-systems."

- Sanford Kwinter

Soft Systems (1993) 


\section{Complexity \& The Edge of Chaos}

Systems-based thinking provides a framework to understand nature and our relationship to it. With a holistic systems-based view of the world and its interconnected subsystems and processes, the dynamic complexity of our environment becomes apparent. This is especially clear when, within this system, one considers the individual as an intelligent being with agency and free will. As such, practices based on determinism can no longer be appropriate, although its history's shadow remains over architecture. The ecological system is an uncertain space, but the biggest source of uncertainty for architecture comes from the social system and the individual. This uncertainty in the form of multiplicity of interactions and agents leads to complex behaviour. The study of complexity has long been engaged by scientists and even non-scientists in the quest to understand the natural and social world.

Complex systems are dynamic systems sensitive to initial conditions that evolve over time utilizing feedback of its processes which generate emergent behaviour. It is emergence, a process of evolution informed by internal and environment system variables, that is responsible for the development of new forms and behaviour. The results of these transitions are usually a more robust state of higher complexity. What is striking however, is that these emergent properties cannot be deduced from its components or subsystems (Weinstock, 2010, p. 31). In other words, these systems are inherently uncertain resulting in unique responses that emerge unpredictably.

What is of particular interest is the 'critical threshold' at which dramatic transformation (reorganization or collapse) occurs. It is in this state of maximum complexity and connectivity between its subunits, between order and chaos, that it is optimized for adaptation and information processing. DeLanda suggests that strong or resilient systems are actually ones that are not locked in stability, but rather are ones that are at that threshold of change (criticality) where it is extremely sensitive and ready to reconfigure to new dynamic states of equilibrium. DeLanda, quoting scientists Stuart Kauffman and Christopher Langton, states they are "poised at the edge of chaos" (1992, p. 159). Interestingly, complex systems possess the ability to self-organize, without the need to tune any adjustable external parameter, to seek out the critical state (self-organized criticality) on its own.

Complex processes are responsible for the formation and evolution of all forms within natural and cultural systems. Forms in nature "are not created by a single force or event, nor by the simple coexistence of any parts; it is the interactions of each part to its immediate surroundings that initiates processes that over time produce coherent forms" (Weinstock, 2010, p. 18). These forms and processes exhibit a high resilience, as such, many of these properties are desirable for an architecture of change and uncertainty. It is with this that we turn to these systems to provide a model for architecture in hopes of operating in harmony with the changing social and natural world. 
A complex architectural system will be:

- Dynamic - Systems are capable of change

- Emergent - Changes stem from feedback and are not predefined

- Self-organized - Strive towards criticality i.e. sensitive to change

In essence, as James Kay states: "complexity is characterized by situations where several different coherent future scenarios are possible, each of which may be desirable, all of which have an inherent irreducible uncertainty as to the likelihood for their actually coming about" (2008, p. 3).

Architecture as a social system then, is also composed of subsystems. These are partitioned here into the material (physical/formal) subsystem and the immaterial subsystem consisting in particular its processes/ practices. By embracing uncertainty and a stronger bottom-up approach to architecture's subsystems, fundamental questions will be confronted. How do we translate systems thinking into action? How can architects propose a design strategy that refers to uncertainty without being deterministic? With this, the system of architecture will be critically poised at the edge of chaos, ready for reorganization and evolution.

\section{Material}

Fluid:

- A substance that has no fixed shape and yields easily to external pressure;

- Not settled or stable; likely or able to change.

(“Fluid," n.d.)

\section{The Complicated and the Complex}

It is important here to distinguish between the complicated and the complex. Complicated architectural forms are prevalent with the progress of technology, seen in the multi-layered assemblages of discrete objects and geometries. These systems, however visually, or compositionally complicated do not necessarily utilize actual complex feedback and performance. In fact, often times it is the seemingly simple that yield complexity. In life, Kiel Moe notes that complexity emerges when "the most archaic molecules happened into each other and synthesized, setting off a set of catalytic reactions and developments leading to all forms of life" (2013, p. 84). Basic units or components provide ambiguity that can invite the greatest of possibilities. 
An analysis of Herzog and de Meuron's 'Bird's Nest', which appears complex with its unordered and non-hierarchical structure, reveals that the complicated nest façade merely masks regularly spaced primary arches (Keller \& Jaeger, 2016, p. 28) (Figure 5). In another example Kentaro Tsubaki, quoting Frank Gehry, describes how the sculptural forms of the Walt Disney Concert Hall was achievable through the latest digital tools:

CATIA also allowed extremely complicated steel to go together on the site without the kind of problems that happen on similar sized buildings. Due to the consistency of information and the precision of the calculations, every element tied back to an origin. When an Ironworker was on the scaffolding, he could get someone to survey him a point and know he was within an eighth of an inch (2012, p. 191).

In this process, highly specific components fit together in a predetermined fashion in precisely one way to produce in actuality, a complicated building that stifles true complexity in its physical system. The weakness in these examples is that a failure in one part of the system will eventually result in the failure of the whole system whether it be in a structural or aesthetics sense.

Careful planning and execution characterizes the work of architects. Although the imagery of complexity is often embraced, they have persistently resisted the undetermined to enter their design processes. Contingency is precluded because it invites the possibility of chaos and disorder which is in contradiction to the closely held association of design with order(-ing): "to design is to give order, and this seems to mean to eliminate chance" (Keller \& Jaeger, 2016, p. 28). Yeoryia Manolopoulou in Architectures of Chance further elaborates:

The more we are aware of the chance universe, the more we want to remove uncertainty, to predict and 'design' chance. The greater the level of indeterminism in our understanding of the world, the more we expect control and the less we expect freedom. In this context architecture's improved knowledge of the indeterminacies of nature is combined with an increasing and excessive tendency to want to control the environment through technological means. (2013, p. xviii)

The result, is the assembly of materials into crystalized forms that are overly prescribed or determined, which entrap its users and suppress the creative forces of agency and the flows of the environment. It becomes a rigid, closed system that fails to accept feedback. 


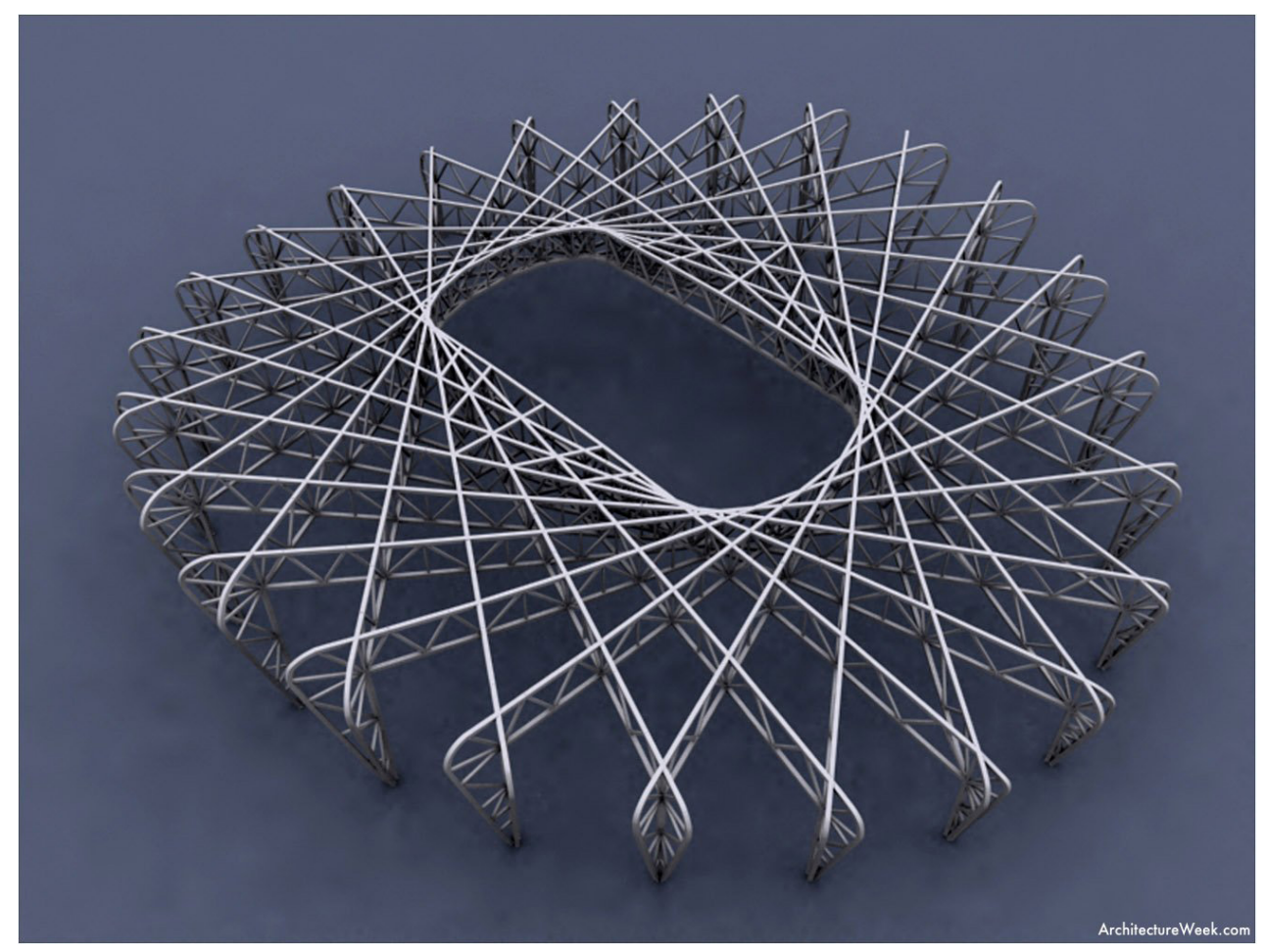

Figure 5. Primary steel structure for the 'Bird's Nest'.

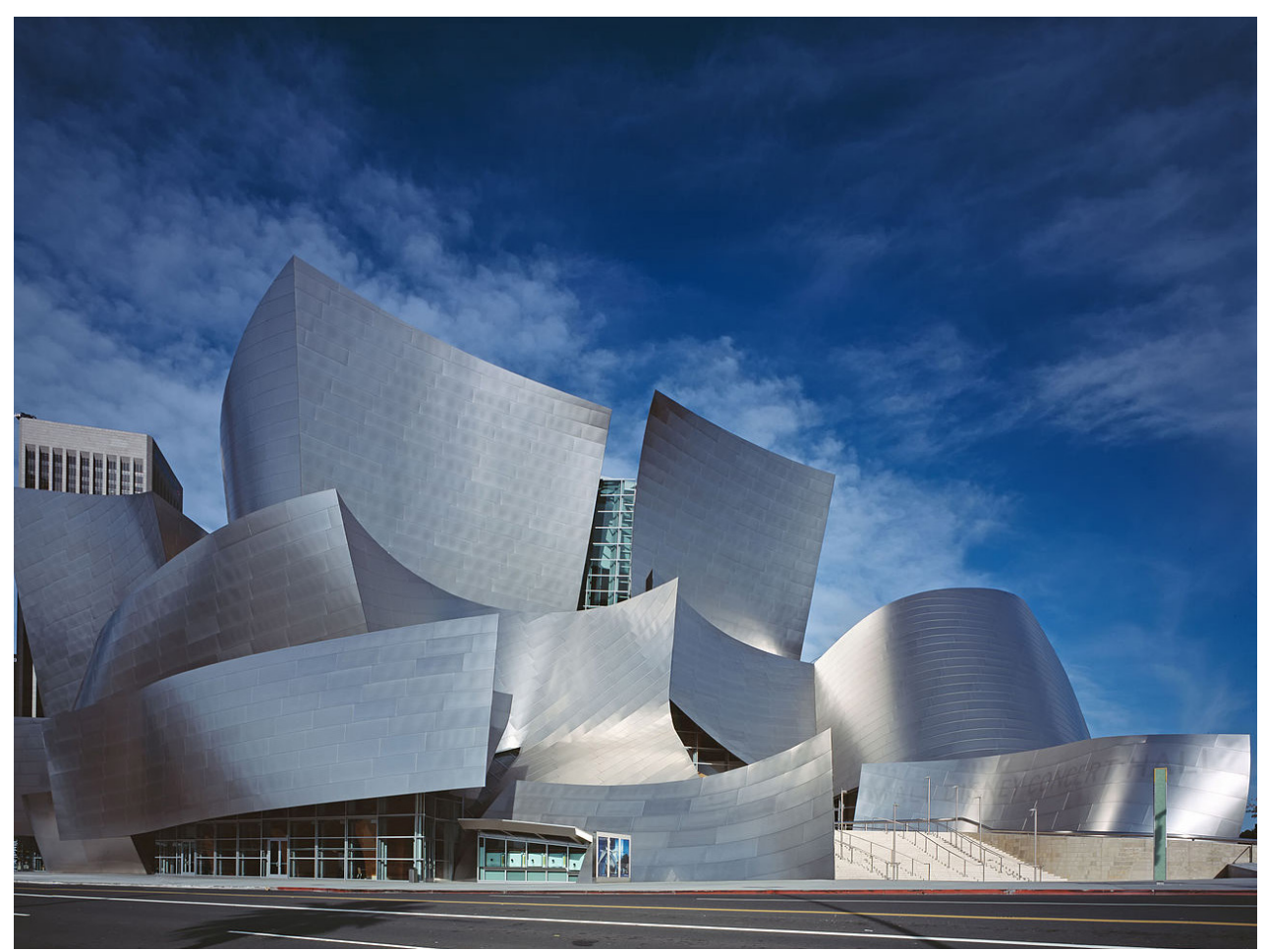

Figure 6. Walt Disney Concert Hall. 2005 
What is suggested is not the elimination of design, but a practice is required that entails the understanding and negotiation of material and systems in a way that exploits its underlying intelligence and material agencies to respond to structural or spatial contexts on their own. Materials capable of complex self-organization is the critical interface for architecture to engage the unknowns of the environment and human agency. This interaction engenders a feedback of alteration and engagement.

\section{Fluidity}

In searching for such materials and systems capable of responding to change, Delanda provides a hint. His suggestion to current society is to "find the right viscosity for our fluxes, the exact consistency that would allow humanity to self-organize" (1992, p. 155). Framing change as phase transitions of matter, he states that the solid state is too ordered, while gaseous state is too chaotic and that the liquid state has the most interesting dynamics. So, the "edge of chaos", where self-organizing effects are maximized, is at the solid-liquid bifurcation (DeLanda, 1992, p. 159). Bauman uses the concept of fluids as a metaphor for change in our social condition:

Fluids, so to speak, neither fix space nor bind time. While solids have clear spatial dimensions but neutralize the impact, and thus downgrade the significance, of time (effectively resist its flow or render it irrelevant), fluids do not keep to any shape for long and are constantly ready (and prone) to change it; and so for them it is the flow of time that counts, more than the space they happen to occupy: that space, after all, they fill but 'for a moment'. In a sense, solids cancel time; for liquids, on the contrary, it is mostly time that matters. (2012, p. 2)

Materially, Solà-Morales in Liquid Architecture shares a similar idea:

We can no longer think only in terms of solid, steady precincts established by lasting materials, but must consider fluid, changing forms capable of incorporating; making physical substance no with the stable but with the changing; no search for a fixed and permanent definition of space but give physical form to time; experience a durability in change completely different from the defiance of time that characterized the classical method (Solà-Morales, 1998, p. 38).

Solà-Morales continues and identifies three states of architecture: solid, viscous, and liquid and its distinct material conditions: firmness, ductility, and fluidity. These states and attributes correspond to three categories: space, process, and time respectively, and is to show the shifting sequence from the traditional to a new structural mode in architecture.

The ideal material thus would possess fluid properties; a substance that has no fixed shape and yields easily to external pressure and thus is likely or able to change ("Fluid," n.d.). But, of course physically, liquids can hardly be seen as relevant for architectural application; this material would have to be 
unique, possessing the right composition and balance of properties. An architectural material conceived as such will enable physical adaptation, unsettling the way in which we live in structures, but also the practices of architecture. An architecture that is fluid is not oriented toward representation nor focused on the space occupied (solid or form), but on time-the event and experience-and thus accepts the contingent.

Through the lineage that outlined the mechanical and soft solutions there has yet to be an ideal material with these optimal qualities that come close to begin to address real, practical issues of application. Mechanical technologies as seen in the Fun Palace, offers a predictable range of configuration that is still largely prescribed. As for softs, the natural material has been inflatables, but as Negroponte proclaimed, "not everybody wants to live in a balloon" (1975, p. 147). Nature and one of its most refined processes: evolution, naturally becomes a powerful source of inspiration in the same spirit of the late Frei Otto's work with structures in nature.

Underlying this type of complex material system is the premise that the properties of the material as a whole will rely the on interactions of components yet not solely from the sum of the parts because it will need to be emergent. It prompts a reconsideration of design. How does one design with this if design implies determination which contradicts emergence? There is a type of weak emergence that provides promise. Such a system is in principle deterministic, but due to its sensitivity to initial conditions, small difference in change produces non-linear behaviour that can have wildly divergent outcomes. To adapt to uncertainty requires a degree of uncertainty be "designed" into the material system. This explored in Part 3.

\section{Immaterial}

A fluid, complex material system open to feedback and exposed to the agencies of its environment will necessarily acknowledge the input from its users. If our perspective is expanded to consider now the convergence of the once discreet systems of material and user, what emerges is a more integrated and robust architectural system. Complexity is multiplied through this coupling, with the system for one process becoming the environment of another and vice versa.

The involvement of the user in the production of architecture challenges a number of its immaterial structures. First, the very concept of an empowered individual shakes the long preconceived image of the user established by architects. This has implications for the process of design and construction. Second, this in turn questions the architect's authority and control. 


\section{The User}

The user in architecture, Jonathan Hill claims, has a hierarchical relationship with the architect that has persisted in history. This was particularly strong during the modern movement, but it continues to extend into discourse today. This relationship is characterized by various strategies to deny or control the user by architects that suggests "a manageable and passive user, unable to transform use, space and meaning" (Hill, 2003, p. 10). Further, even the term user, he concludes, has negative connotations (Hill, 2003, p. 27) and creates an abstraction that assumes all users are the same and have the same needs. An acknowledgement of the user as unique affords them agency and problematically for architects, it invites the messy and the wild: the uncertain.

Hill contends that how the user is conceptualized affects what is designed. While "passive and reactive users are dependent upon existing conditions, which they are unable to fundamentally change" (Hill, 2003, p. 88), accepting a creative user, one enabled to conceptually or physically alter or create new space, implies a dynamic, responsive, and indeterminate architecture. Hill observes that, "when the designers are also the users the building process has the potential to be not the production of a fixed object but an endless, flowing cycle of designing, making and using" (2003, p. 58). Manolopoulou underscores the importance of this:

While deterministic applications of chance result in fixed forms because they are fully defined by the actions of the author, indeterminate applications of chance are open to the actions of the performers-users who invent ever-changing scores and spaces. This radical and unpredictable condition of chance is critical and deeply social, placing emphasis on how buildings perform rather than how they appear as finished objects. (Manolopoulou, 2013, p. xxiii)

Fundamental to this discussion is the recognition that architecture as a social product necessarily requires the social and personal component-that is, architecture is co-produced by both architect and user, through both design and agency. Once architecture is realized it belongs to the cultural and social domain and thus will be subjected to the agency of people. Design is therefore not complete upon construction.

\section{Control}

Most apparent attempts to define and diminish the user are found in functionalist ideas. The idea that functions can rationally deduce ideal forms by extension implies a deterministic principle that the actions of users are predictable and every event has a cause. The user is abstracted with constant and universal needs. Closely associated with modernism and industrial advancement, Hill highlights the sentiments of the time: "The paradigmatic form of the body is the technician at work in the factory 
and the home. The passive user learns to operate a space the way the technician learns to operate a machine-the correct way" (Hill, 2003, p. 17).

Hill points also to another relationship between architect and user. An analogy of user to actor he notes is witnessed by Beatriz Colomina in houses by Adolf Loos and Le Corbusier (Hill, 2003, p. 18). This suggests the relationship of architect to user is that of director to directed. It is common for an architect to supply narratives and frame experiences in describing their building as if the same experiences and emotions is shared by all users that also conform to the vision of the architect's (Hill, 2003, p. 18).

Hill argues that a creative user is empowered with agency to freely change or even create new spaces. This idea of empowerment is not only about allowing users to take control of their environments post-occupation, but also participate within the design process. However, this will undeniably be seen as a threat to the role and the expertise of the architect as master of form, building and human environments, thus challenging the notion that only architects can make architecture. It would require that architects give up control which, they have strongly resisted.

A new approach to the relationship between architect, user and material, where the struggle for control is renounced, would allow each party an opportunity to participate in the process and evolution of architecture within society and nature. This approach admits a certain level of uncertainty or different types of chance as Manolopoulou would characterize. One instance is active chance:

[it] accepts the aleatory as a critical part of all architectures, irrespective of place, period and style. It asks how buildings and places perform in time and society, rather than how they are as objects. This is a social kind of chance, underlined by non-finality and the possibilities of co-making. It values the inhabitants of architectures as co-designers and generally accepts all performative expressions of everyday chance. (2013, p. 223)

It also means to accept the fact that "the perception and use of buildings are never entirely controllable" (Manolopoulou, 2013, p. 193). 
for

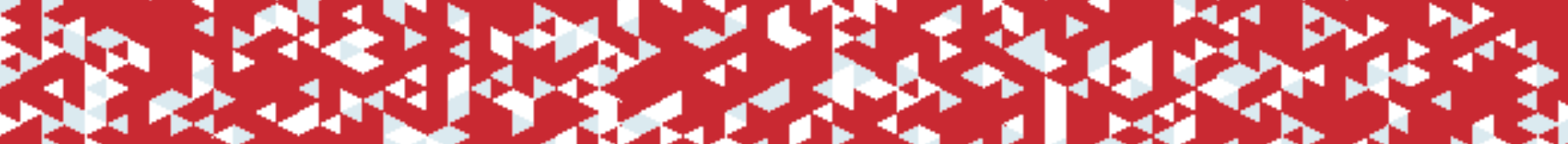

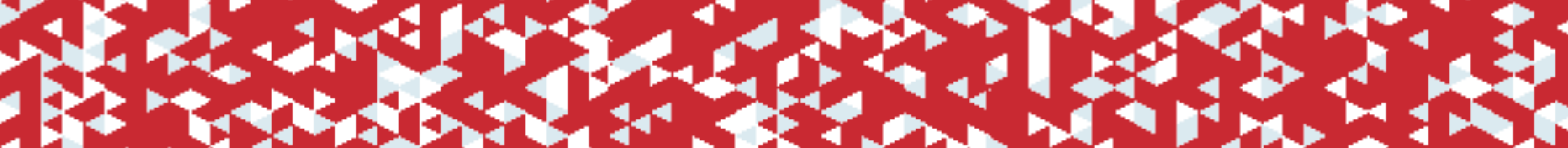

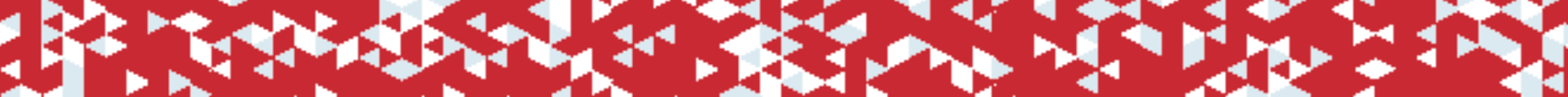

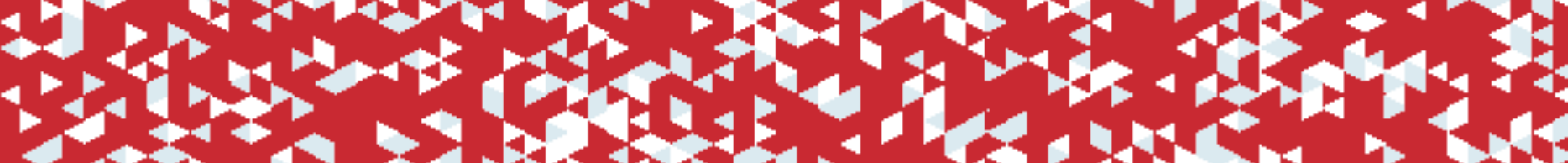

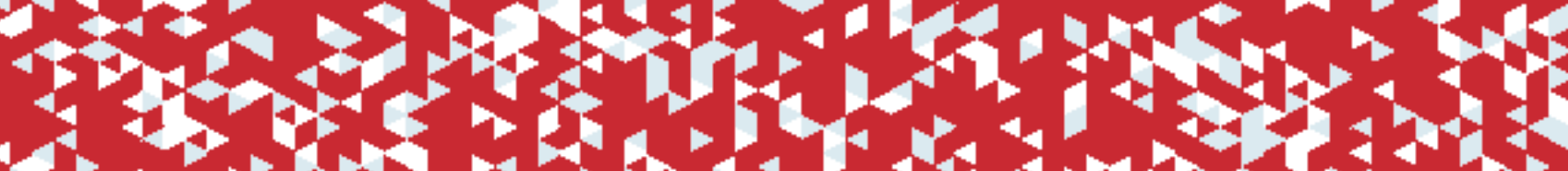
and S

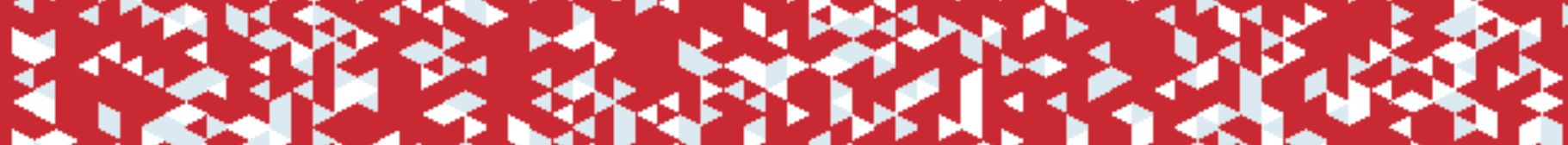

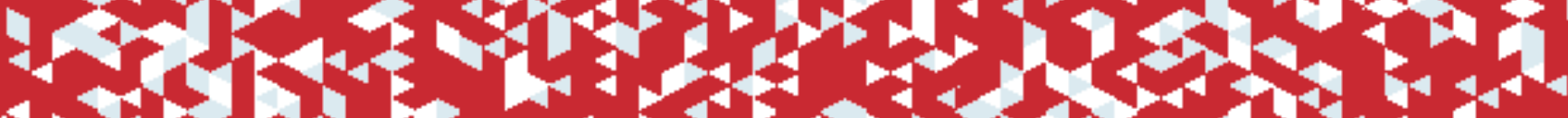

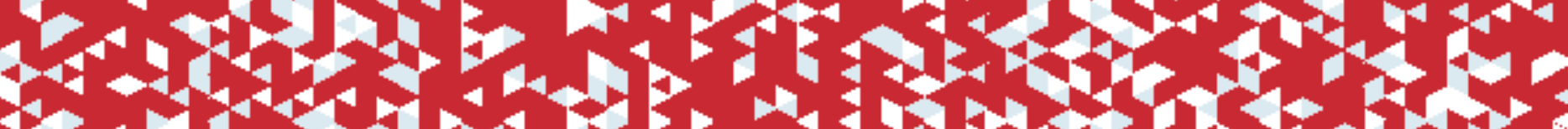

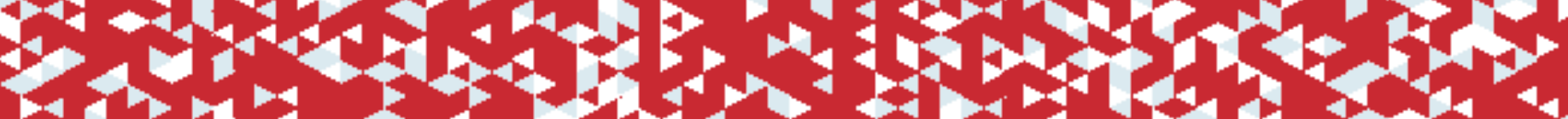

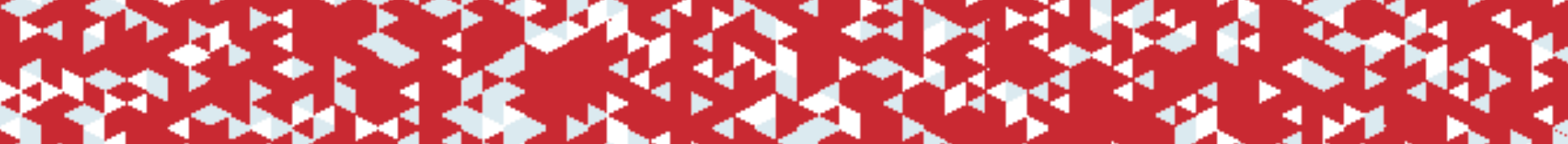

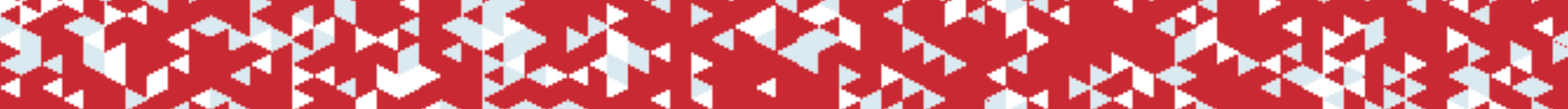

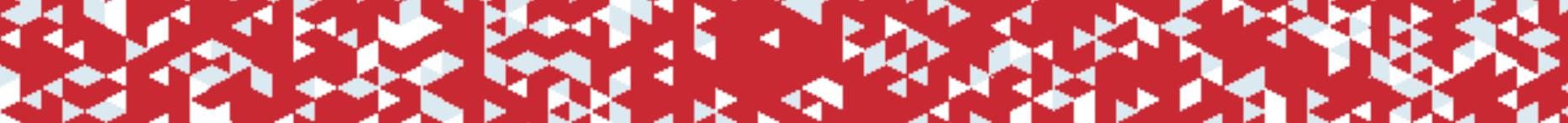

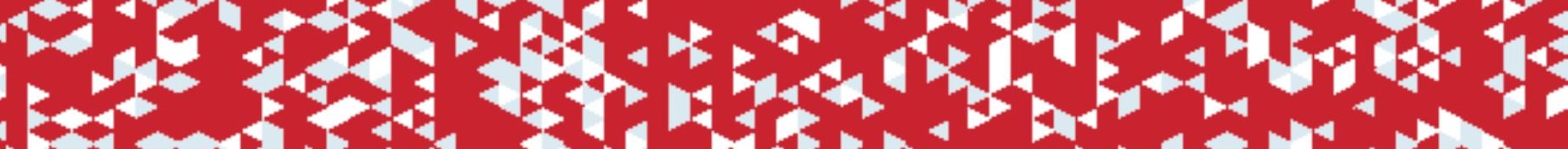

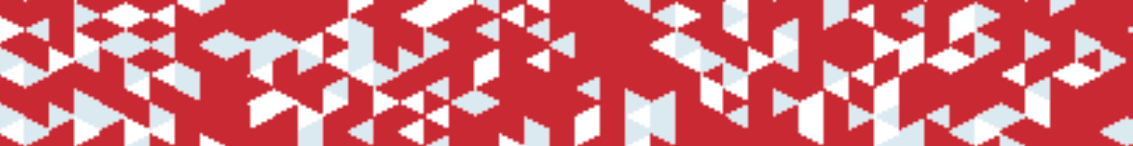

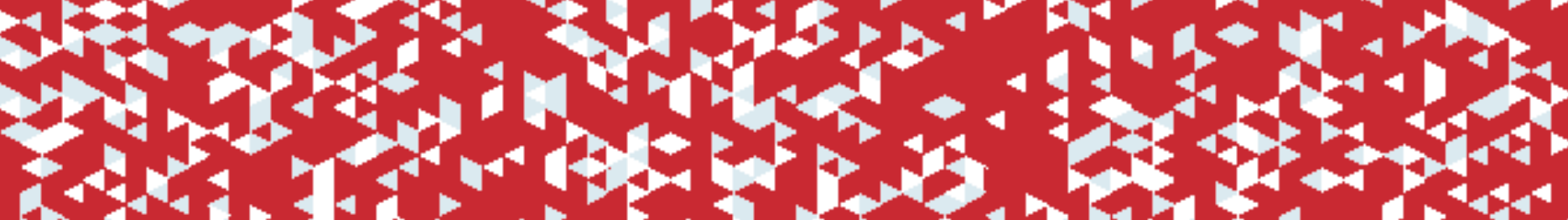

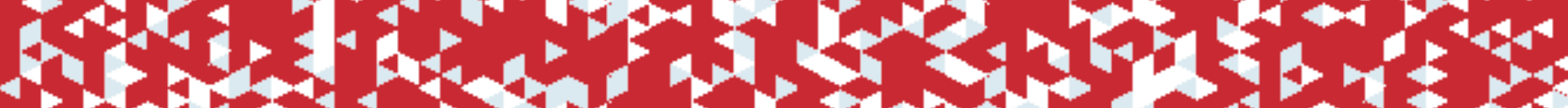
Cotor.

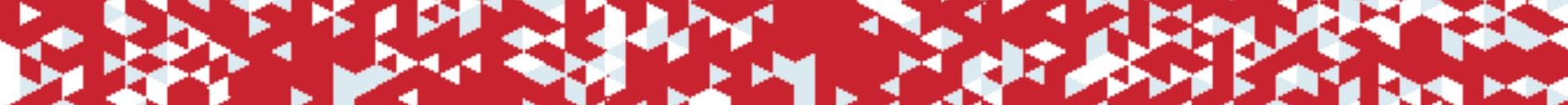

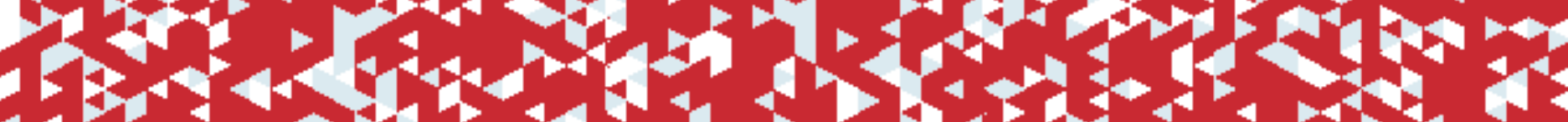

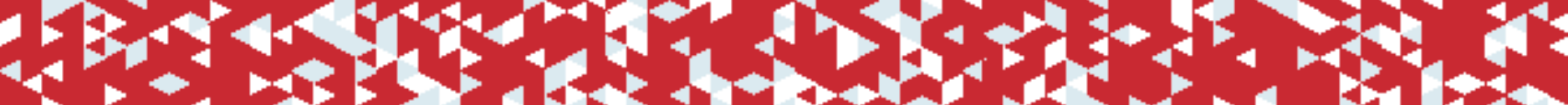

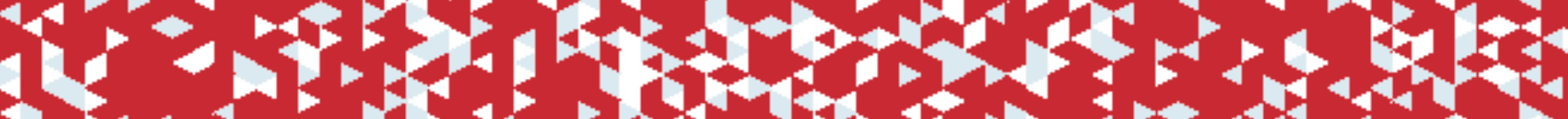

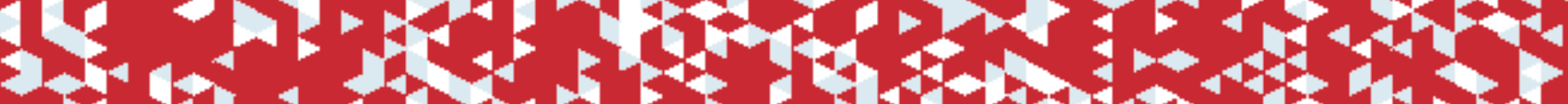

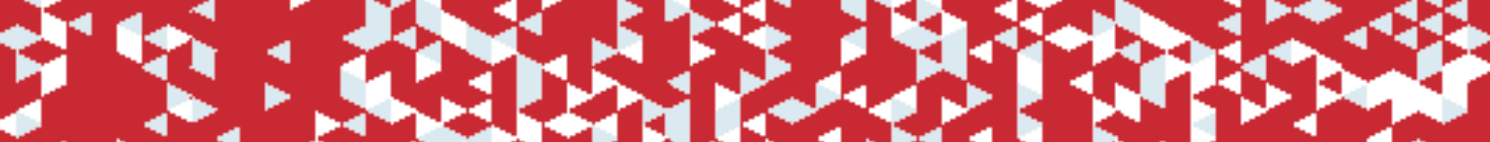
4.4 


\section{PART 3: MATERIAL ARCHITECTURES}

"We are beginning to recover a certain philosophical respect for the inherent morphogenetic potential of all materials. And we may now be in a position to think about the origin of form and structure, not as something imposed from the outside on an inert matter, not as a hierarchical command from above as in an assembly line, but as something that may come from within the materials, a form that we tease out of those materials as we allow them to have their say in the structures we create."

- Manuel DeLanda

Material Complexity (2004) 


\section{Granular Matter}

The trajectory of adaptable architecture is rapidly moving towards the microscopic — engaging the fields of nanotechnology and bioengineering through the rise of small modular robotics and smart materials. There exists however, a category of macroscopic materials known as granular materials that hold much potential and are only beginning to be considered for architecture. Granular matter is defined as large amounts macroscopic elements in loose contact. In fact, they are "ubiquitous in nature and are the second-most manipulated material in industry" (Patrick, Nicodemi, Delannay, Ribière, \& Bideau, 2005, p. 121). Granular material encompasses a vast range of materials with different particle types and sizes, from fine sand to large boulders.

\section{Sand and Snowflakes}

In natural granular materials, such as sand, the particles are typically simple and passive, but as a collective system, their loose, fictional contact give rise to special behaviours becoming a unique state of matter. Phillip Ball (1999), explains the patterns and formation of granular heaps. Although individual grains are composed of solid material, granular substances are able to flow like a liquid through a behavior called liquefaction yet are able to resist shear stress and can support loads. The organization of granular material seem to exhibit behavior seen in chaotic systems, where the smallest perturbation can have unpredictable reactions. What is of particular interest, is that instead of trying to escape the critical state, it seeks to constantly return to this "tipping point". That is, it is remains highly sensitive to change but it is not infinitely unstable, but rather robust. Consider a sand pile, it will grow until it reaches its maximum angle of repose, where additional grains of sand added can trigger an avalanche transferring sand onto adjacent grains, where under the same rule can also trigger an avalanche. In this case, local interactions between simple component parts self-organized towards this state of criticality.

The formation of snow crystals is a highly sensitive process, as Sanford Kwinter describes in Architectures of Time (2001, p. 27), they "[are] literally the product of 'time,' in it growth and design are one." Although each crystal contain a fixed, pre-established molecular structure, it can produce dynamically different forms. Kwinter eloquently elaborates:

What is interesting is that despite its partially fixed matrix no two results are ever alike. Each is different because the crystal maintains its sensitivity both to time and to its complex milieu. Its morphogenetic principle is active and always incomplete (i.e., evolving)-the snowflake interacts with other processes, across both space and time; it belongs to a dynamical, fluvial world. As the snow crystal falls it absorbs, captures, or incarnates all the chance events, all 
the fluctuating conditions (magnetic, gravitational, barometric, electrical, thermal, humidity, speed) and builds them, or rather uses them, to assemble itself, to form its structure or edifice. The snow crystal creates itself in the middle of, and by means of the convergences of, flux. Thus snow crystal morphogenesis is less the result of specific, punctual external causes than a sympathetic but critical insertion within, and the subsequent 'cybernetic' management of, already present flows. This analytical model-based on developmental pathways, dynamical interactions, singular points, and qualitative movements in abstract, sometimes multidimensional space-arguably furnishes a far richer theory of 'site' than most currently employed in orthodox aesthetic or architectural practice. (2001, p. 27)

The allure of the snowflake structure has also inspired product designer Peter Pearce. Pearce finds strategies for design in the structure of nature in the same way D'Arcy Wentworth Thompson writes that "the form of an object is a diagram of forces" (Pearce, 1978, p. xiv). The snowflake, illustrates a concept he calls minimum inventory / maximum diversity where its molecular structure governs certain basic physical, geometrical, and chemical constraints, but is able to achieve maximum (infinite in this case) diversity through its interaction with extrinsic forces such as described by Kwinter (Pearce, 1978, p. xii). This idea becomes a system of standardization and modularity (minimum inventory) that can be combined for the greatest performance, efficiency and ultimately sustainability:

If a building system can be considered analogous to a molecular structure which is highly responsive to varied actions of force, it may offer the real possibility of generating building forms responsive to the human needs and natural requirements of diversity, adaptation, change, and the conservative use of natural resources (Pearce, 1978, p. xiv)

\section{Jamming}

Granular materials exhibit an interesting phenomenon called jamming. Jamming is the phenomenon in which rigidity is achieved in disordered, amorphous systems comprised of many constituent particles that interact via short-ranged repulsive forces (Jaeger, 2015), like in the case of the sand. Jamming informs a unique type of phase transition that does not require temperature but allows the flow of granular matter to solidify in metastable configurations through contact forces. These states are, as previously described, far from equilibrium and sit at the edge of chaos which allow the material to transform reversibly between a solid-like state that can support load and withstand shear, to a liquid-like state that is porous and reconfigurable. It is this key attribute that gives granular systems great potential and appeal architecturally, but also in many other applications as well, such as in harbour breakwaters and foundations valuing its energy dissipating qualities (Jaeger, 2015, p. 13). 


\section{State of the Art: Architectural Granular Material Systems}

In architecture, applications of jamming have emerged in recent years in the construction of load-bearing structures through random aggregation of interlocking granules that jam under gravity without the use of supplementary binding agents or mechanisms. Jammed granular architectural systems is a relatively new field and presents many theoretical and practical challenges. Compounding this is the fact that the material science itself has had longstanding challenges predicting macroscopic aggregate properties (Keller \& Jaeger, 2016, p. 4) and "no rheological laws exist for such materials" (Patrick et al., 2005, p. 121). Statistical descriptions and empirical observations are relied upon.

Granular materials, at an architectural scale, can produce a fluid architecture-a unique system that exhibits properties of a liquid through its re-configurability and free form, but is capable of self-stabilizing structurally, forming a solid-like state. Its ability to go between these states and thus allow for constant formation and reformation of a structure challenges the pervasive notion of permanence in architecture. Additionally, with specifically designed grains, various global behaviours can be programmed upon accumulation. These properties show great promise for an architecture that is able to adapt to variable and uncertain forces. It promises, to reconceptualise architecture's basic building components and its design and construction logics. The following is an overview of development by key contributors.

\section{Precedent: Tumbling Units - Kentaro Tsubaki, 1996}

One of the early originators working with aggregating loose materials in architecture, Kentaro Tsubaki created granular forms he called tumbling units. The units were made from clay. To assemble a structure he utilized sand as a formwork for his model of a dome enclosure. Tsubaki in his project, was investigating the idea of indeterminacy in architecture and looked to introduce "an architectural idea equivalent to 'probability" that changed $19^{\text {th }}$ century science (Tsubaki, 2012, p. 193). This was a critique of the "rather simplistic" use of inexpensive computational power available to achieve "deterministic precision" to facilitate cheaper and faster construction (Tsubaki, 2012, p. 193). He provides the example of the aforementioned Gehry project. Where statistical mechanics prompted a paradigm shift in the sciences, Tsubaki questions whether this can be applied to architecture and what the tectonic implications would be. Through his experiments he concludes that "by conducting a large number of empirical experiments, it is possible to statistically establish a distribution pattern against the overall state of the aggregate system" (Tsubaki, 2012, p. 198) and it is possible to establish and predict "a 'most probable' tectonic characteristic of an indeterminately complex system” (Tsubaki, 2012, p. 199). 


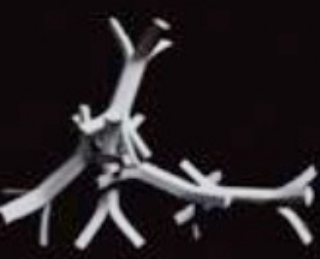

21

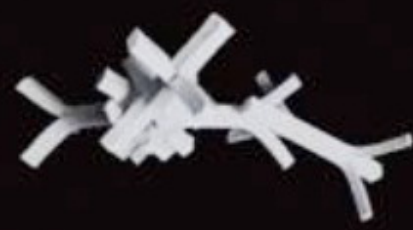

- $21 \mathrm{~A}$
22 ILFORD HPS PLUS

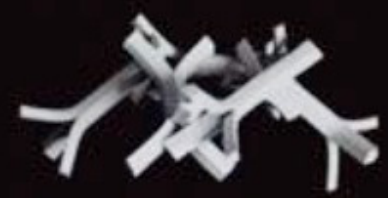

$-$

$22 \mathrm{~A}$ 23
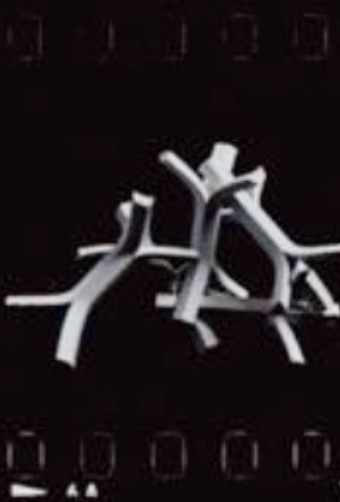

7

\section{ILFORD HPS PLUS}

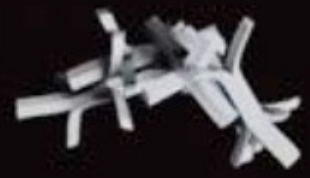

$\begin{array}{llllll}5 n & 8 & 0 & 3^{6} & 4\end{array}$
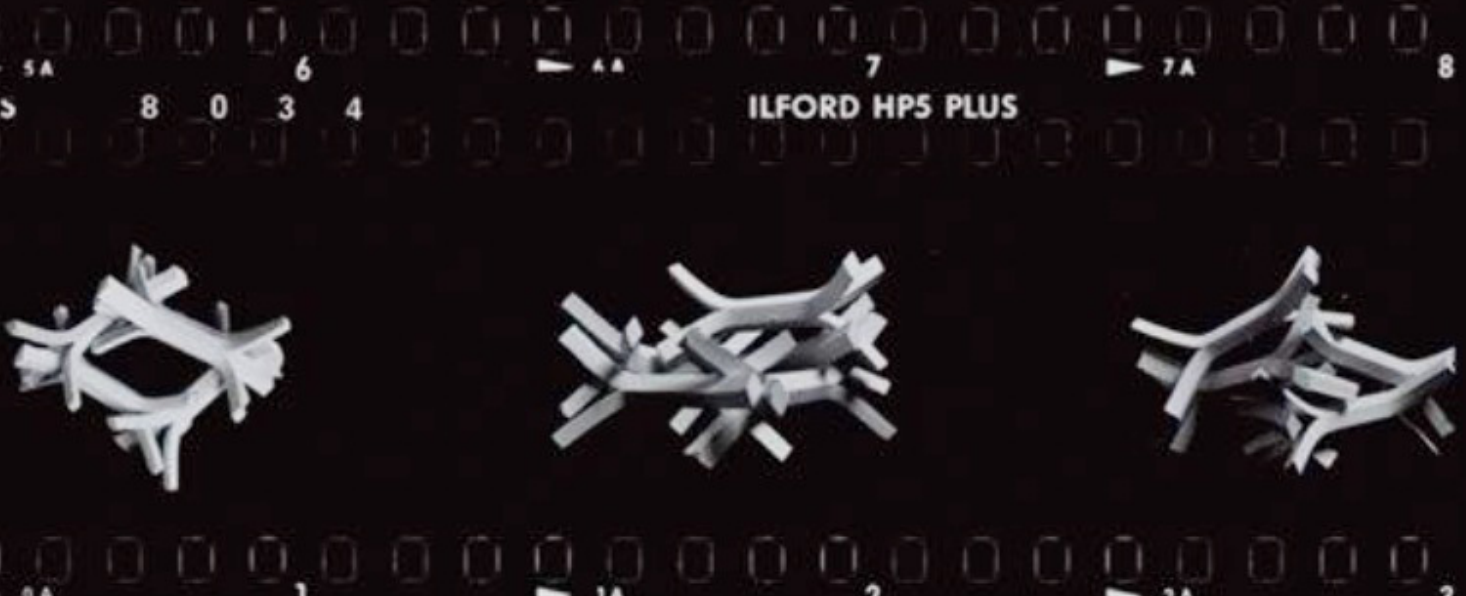

Figure 7. Tumbling Units tectonics. Reprinted from "Tumbling Units, "by K. Tsubaki, 2012, Matter: Material processes in Architectural Production, 196.

On the act of construction, Tsubaki notes the issue of skilled labour/judgment—contrasting the need for precision by minimizing the unknown to reduce skilled on-site judgement, with the indeterminate system of the tumbling units where a similar result can be achieved with much less effort by allowing the system to compute itself and accepting a probabilistic solution (Tsubaki, 2012, p. 199). 


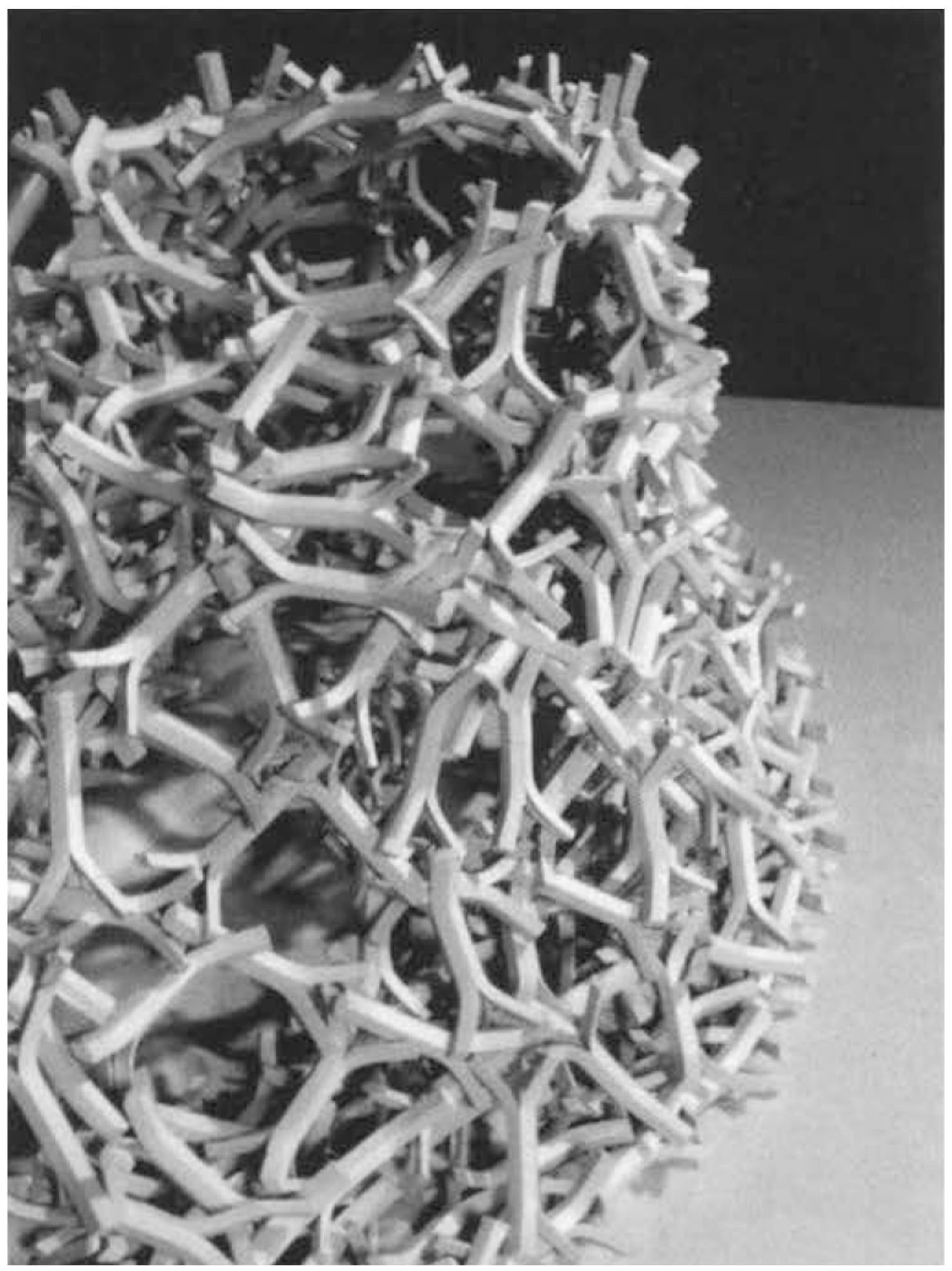

Figure 8. Tumbling Units canopy detail. 


\section{Precedent: Aggregate Architecture - Dierichs + Menges, Institute of Computational Design}

From a material and performative standpoint this concept of an "aggregate architecture" has been furthered in great detail by Karola Dierichs and Achim Menges. Beginning in 2003, Achim Menges and Michael Hensel have directed studies on aggregate structures at the Architecture Association and Rice University (Hensel \& Menges, 2008). Karola Dierichs with Achim Menges now lead architectural research in the field of designed granular matter at the ICD. Through the initial studios conducted, they have been able to synthesize an analysis of the various designed particle morphologies used by looking at six variables: 1) bounding box, 2) the convex hull, 3) overall concave hull, 4) detailed concave hull, 5) plate number, and 6) joining steps (Dierichs \& Menges, 2012b). The performance of the overall granular system is governed by the individual particle and so the morphological catalog produced provides an important reference. However, more detailed experiments they admit, on the scale of a few hundred to tens of thousands of particles are required to verify the relationships between the variables at the particle level and the performance of the overall system. Simulation and digital computation has a strong role in the research and used in conjunction with empirical tests to verify observations (Dierichs \& Menges, 2015).

Until now, particles employed in research were fabricated from non-actuated materials, meaning they do not respond to environmental changes. Recently, smart, actuated particles have been created that are able to respond to environmental parameters such as light, heat, and/or humidity. Their latest hygroscopically reactive particle consisting of bilayer wood composites can curl and form hooks under specific humilities (Dierichs \& Menges, 2016). This can, for example resolve the difficulty of disentan-

gling hook particles or in another scenario, allow particles to be transported flat and then activated to a $3 \mathrm{D}$ form when triggered by heat. These actuated particles greatly advances the concept of programmable matter allowing another level of possibilities. Not only is the overall system able to shift, but now the particle itself is capable of change.

Granular material has been strongly aligned with robotic processes, viewing it as a form of additive manufacturing that allows for precise pouring paths and material deposition (Dierichs \& Menges, 2012b). Paired with sensing technology, the emergent behaviour of a formation can be monitored providing feedback that robots can then be use to precisely respond and make adjustments (Dierichs $\&$ Menges, 2016, p. 6).

The 2015 ICD Aggregate Pavilion consisted of 30,000 injection-moulded particles arranged into large granular columns by cable robots. The columns demonstrate their ability to form a 90 degree angle of repose, and as such, this is one reason for its interest for architecture as the material can start to take on straight forms, and not just slumping piles. 


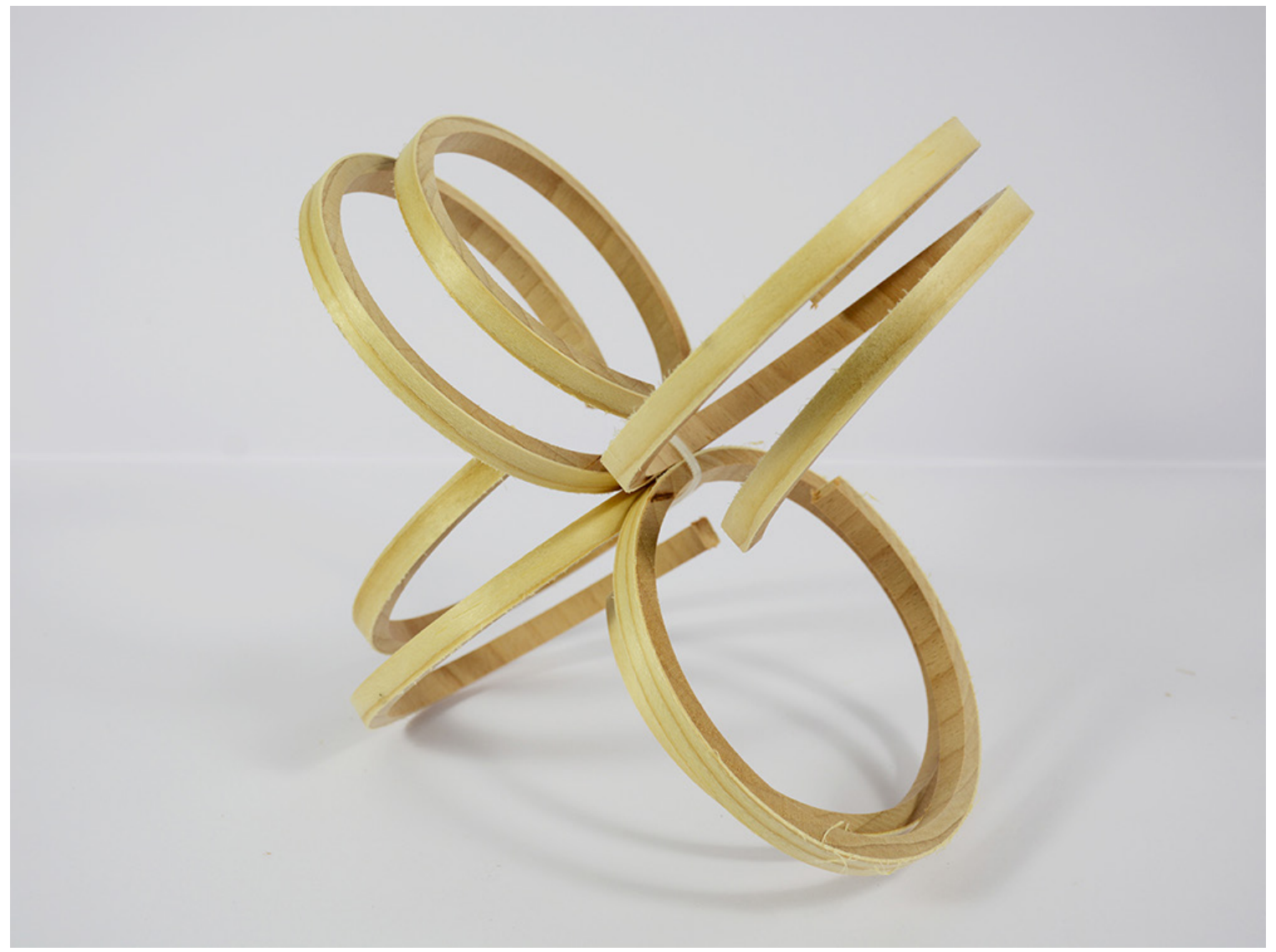

Figure 9. Hygroscopic particle can curl in response to humidity.

36 Architecture at the Edge of Chaos 


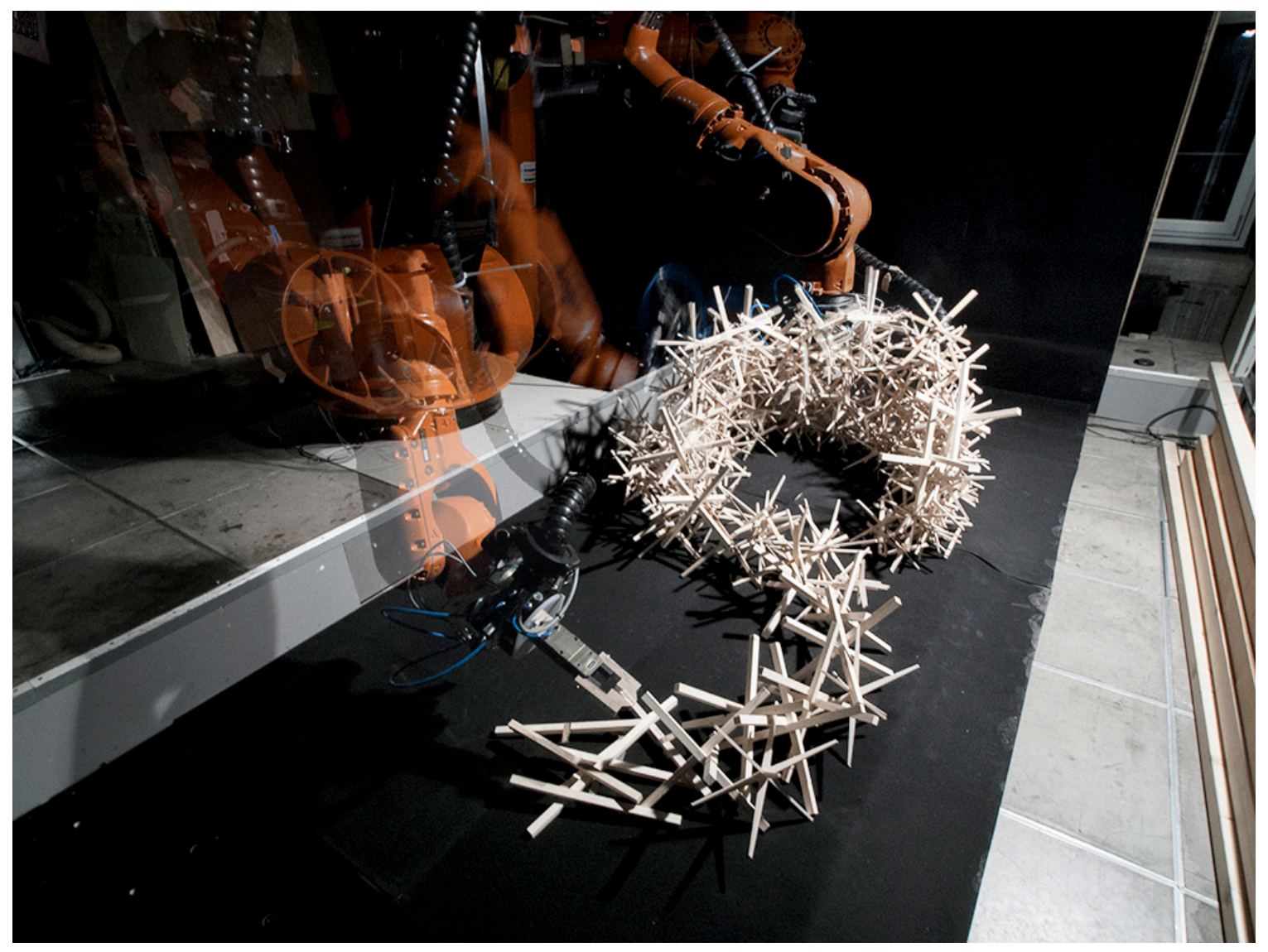

Figure 10. Behavior-based Robotic Strategies for Adaptive Design of Aggregate Systems 

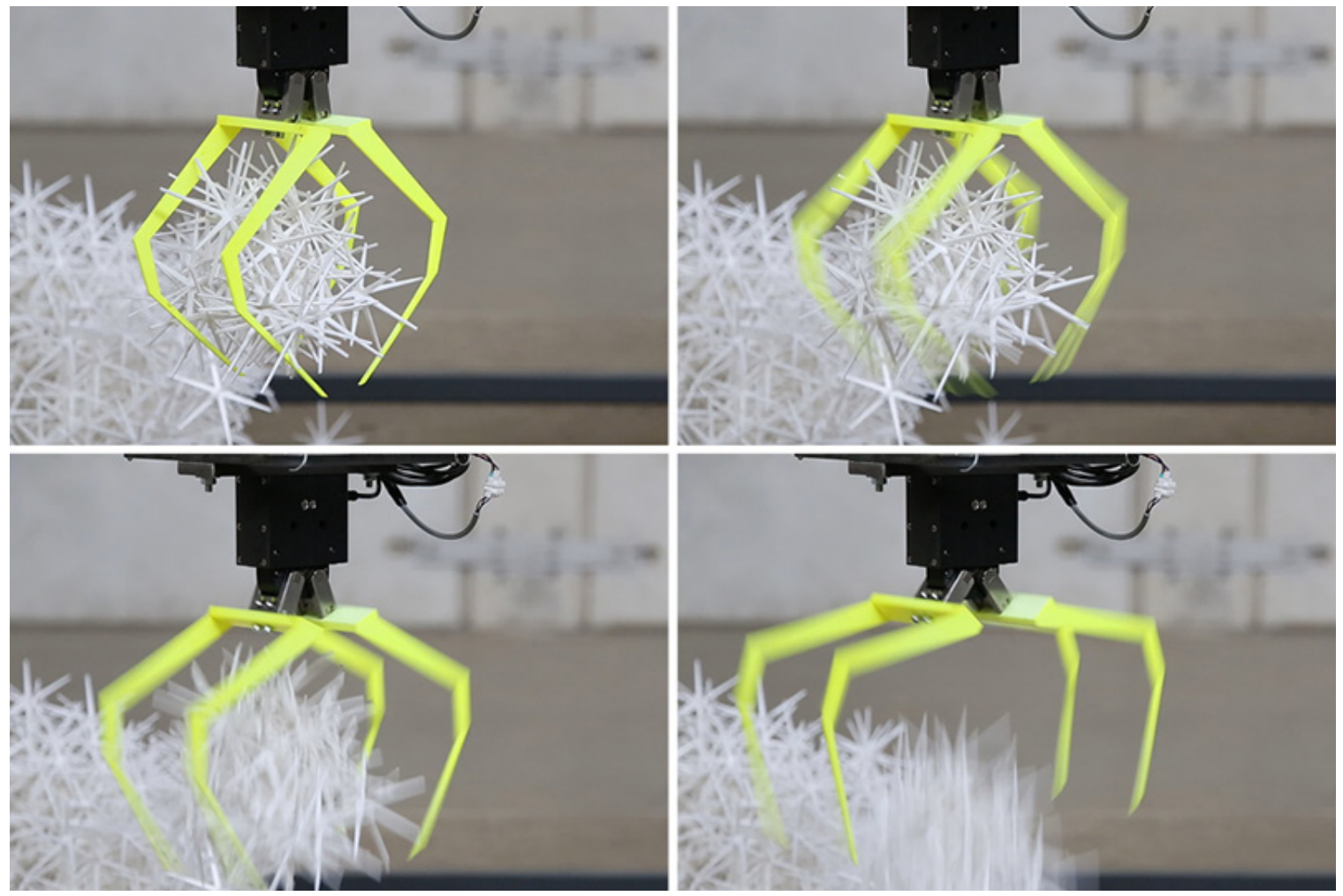

Figure 11. Gripping effector tests with cable robot for Aggregate Pavilion 2015

38 Architecture at the Edge of Chaos 


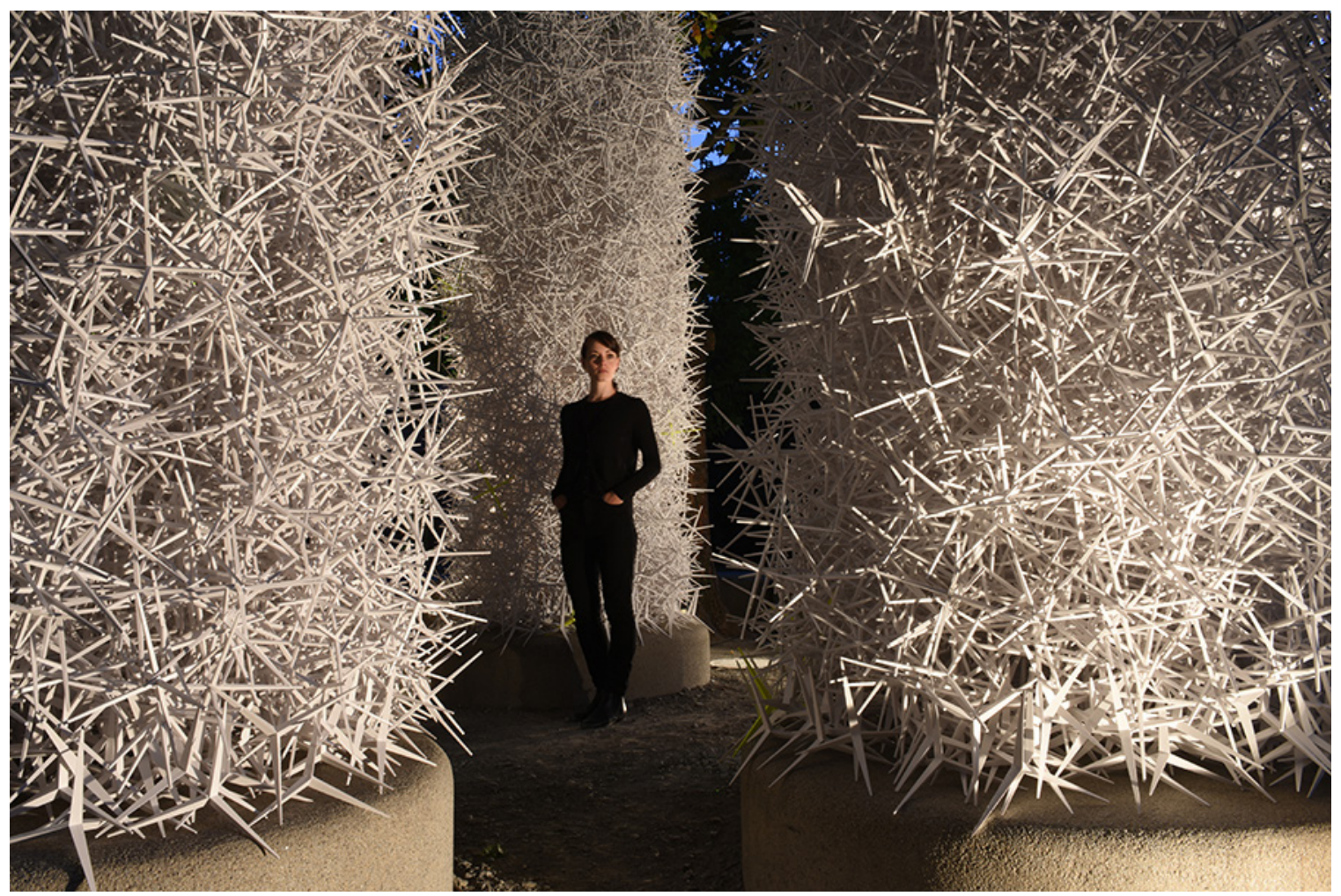

Figure 12. Aggregate Pavilion 2015 


\section{Precedent: Rock Print - Gramazio Kohler Research, ETH Zurich, and Self-Assembly Lab, MIT, 2015}

Another large scale exploration of aggregate systems is seen in the Rock Print project by research labs Gramazio Kohler Research, ETH Zurich and Self-Assembly Lab, MIT presented at the 2015 Chicago Architectural Biennale. It introduces a novel process to aggregate systems. By alternating lightweight, synthetic aggregates (crushed glass that is heated to a foam) with textile filament layered by a robotic arm, a planned sculpture was created with no additional support elements (Lucien, 2015). In this system, the string guided the form, in essence loosely "holding" the desired profiles and then allowing the excess material around it to be shed once the formwork was removed. This presents a way to control granular systems. It also perhaps presents, a possible layer of structural redundancy if hybridized with a specific designed granulate system while being able to maintain the system's unbound property.

In their research they are examining tensile reinforcement through lamination of different fabrics/ string and very much robotic fabrication. It is viewed as a critical tool necessary for advance design and manipulation processes required of bulk materials and granular aggregations in order to achieve a precise, repeatable, and informed process (Aejmelaeus-Lindström, Willmann, Tibbits, Gramazio, \& Kohler, 2016).

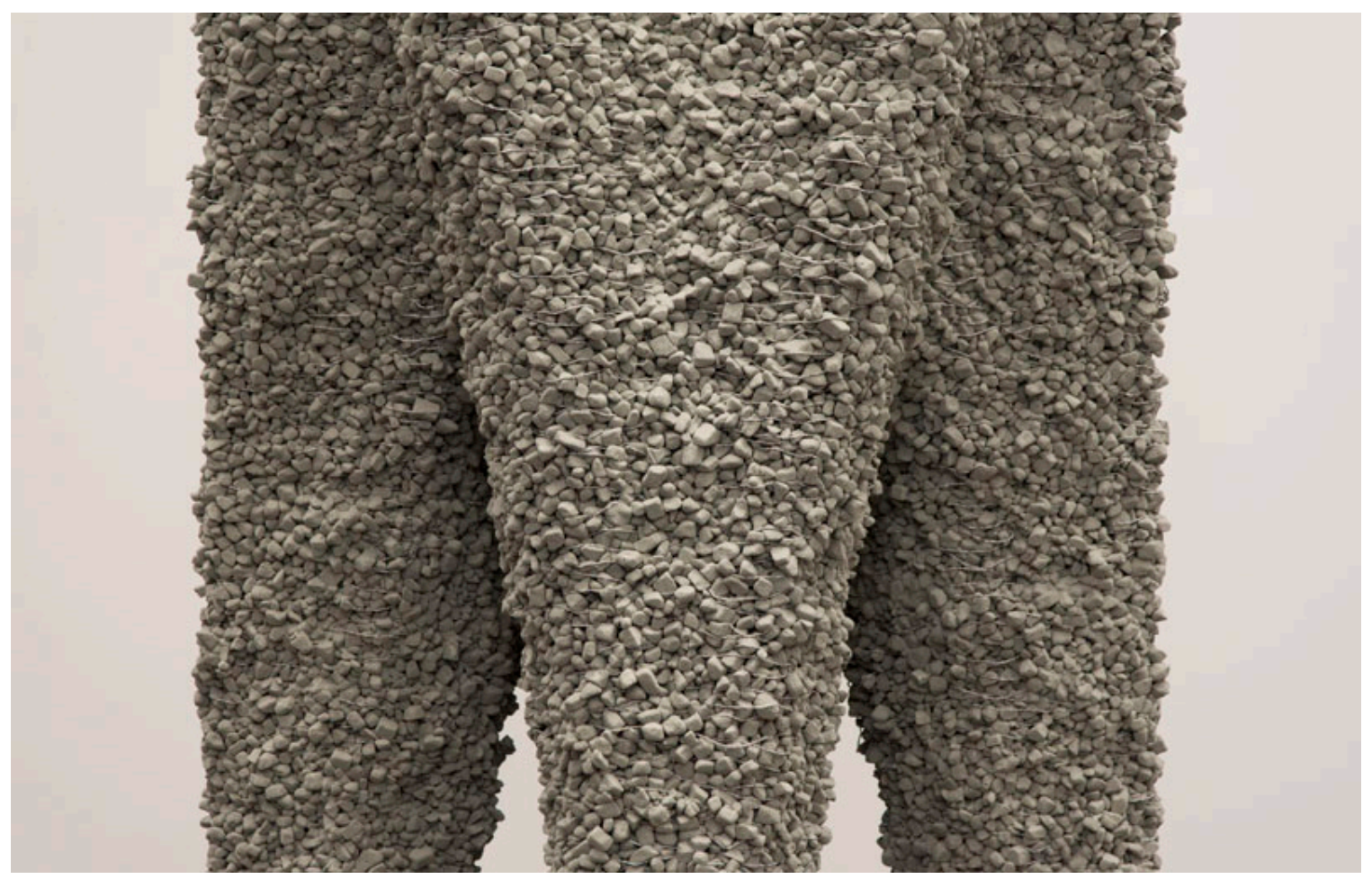

Figure 13. Close up of Rock Print 


\section{Summary}

The state of the art reveals a heavy importance placed on construction processes. In the case with granular systems, the aggregation process and methods has a great impact on the outcome of the structure. Each successive move is strongly dependent on the states of previous moves prompting the need for continuous observation and feedback. Precision has thus emerged as an important factor in assembly and thus has naturally pushed robotic technologies into the discourse, where reliability and repeatability can be best controlled. Together with the development of complex, composite particles that actuate, the trajectory appears to be moving towards greater more specialized and thus exclusive means. This creates a tension with the apparent simplicity and accessibility of the aggregation process that utilizes relatively simple or standard granular media and low skill that the author feels is the strongest feature that should be embraced. It is towards this notion that the thesis orients its experiments and explorations.

Architectural granular systems is a transdisciplinary undertaking requiring the contribution of diverse knowledge. Attesting to this, the May 2016 issue of the scientific journal Granular Matter, devoted a special Topical Collection entitled Jamming-Based Aleatory Architectures that focused on the intersection of granular materials research and architecture. Some of the findings addressed in the issue will now be discussed though the following material research.

\section{Material Research}

Granular behaviour can only be reliably investigated through physical, empirical testing. The current state of research is primarily focused on technical and performance properties of the material and can be categorized in under two groups: 1) grain morphology and behaviour of the aggregate system and 2) aggregation processes (Dierichs \& Menges, 2016). Studies in grain morphologies include looking at geometry and material composition of the individual grain and its effect on the behaviour of the aggregated mass. Geometry and material (friction) largely effects its jamming capabilities and thus structural behaviour. Aggregation methods or construction techniques encompass process, control and methods of affecting the system. The following material research provides a general overview of the behaviour of designed granular systems in both categories through empirical experiments.

In the context of this thesis, the goal of the material research was to examine and demonstrate several factors: stability, malleability, its ability to go from one state to the other, and how to control such a material. To begin to serve as architectural elements stability is necessary to retain its form and withstand loads. Malleability is desired in order to permit a freedom of manipulation and forms. The key for a successful adaptable system will be its ability to shift between solid and liquid states- to not be too frozen, rendering it immutable, or too liquid and uncontrollable. 
Figure 14 outlines the chronology of the material research. Committing to a specific particle morphology, aggregation processes were the first set of experiments conducted. This led to its application in several design explorations. The material research was concluded by investigating the differentiating effects of particle morphology and verifying/ formalizing many of the observations found through the earlier explorations. The following however, presents the research starting at the particle scale with morphological studies proceeding to processes then to applications.

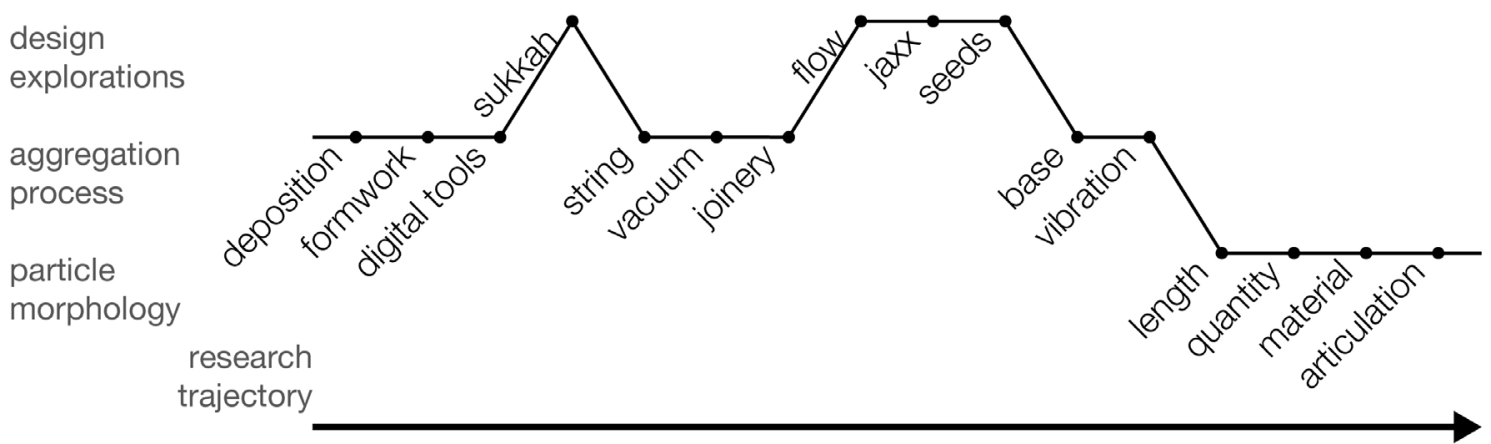

Figure 14. Sequence of research experiments

Initial materials sampling and exploration was conducted by primarily recycling found material of large quantities. Cut binder coils and wood off-cuts were readily accessible and available in a sizable quantity, however it was immediately found that greater design and intention in the base component was necessary to achieve something voluminous and stable.

A general particle morphology, under the considerations of fabrication time and cost, ease of assembly, and performance, was adopted for testing aggregation processes and then used as the base reference module for morphology tests. $3 \mathrm{~mm}$ sheets of MDF (an engineered wood composite) were used to create crosses that were assembled via a slot joint to create 3D star-shaped geometries (Figure 15). The laser cut constituent shapes were all identical for ease of assembly. The arms were tapered to allow for a greater chance for interlocking. The resultant morphology was an eight-armed particle measuring 100 $\mathrm{mm}$ in its longest dimension. 


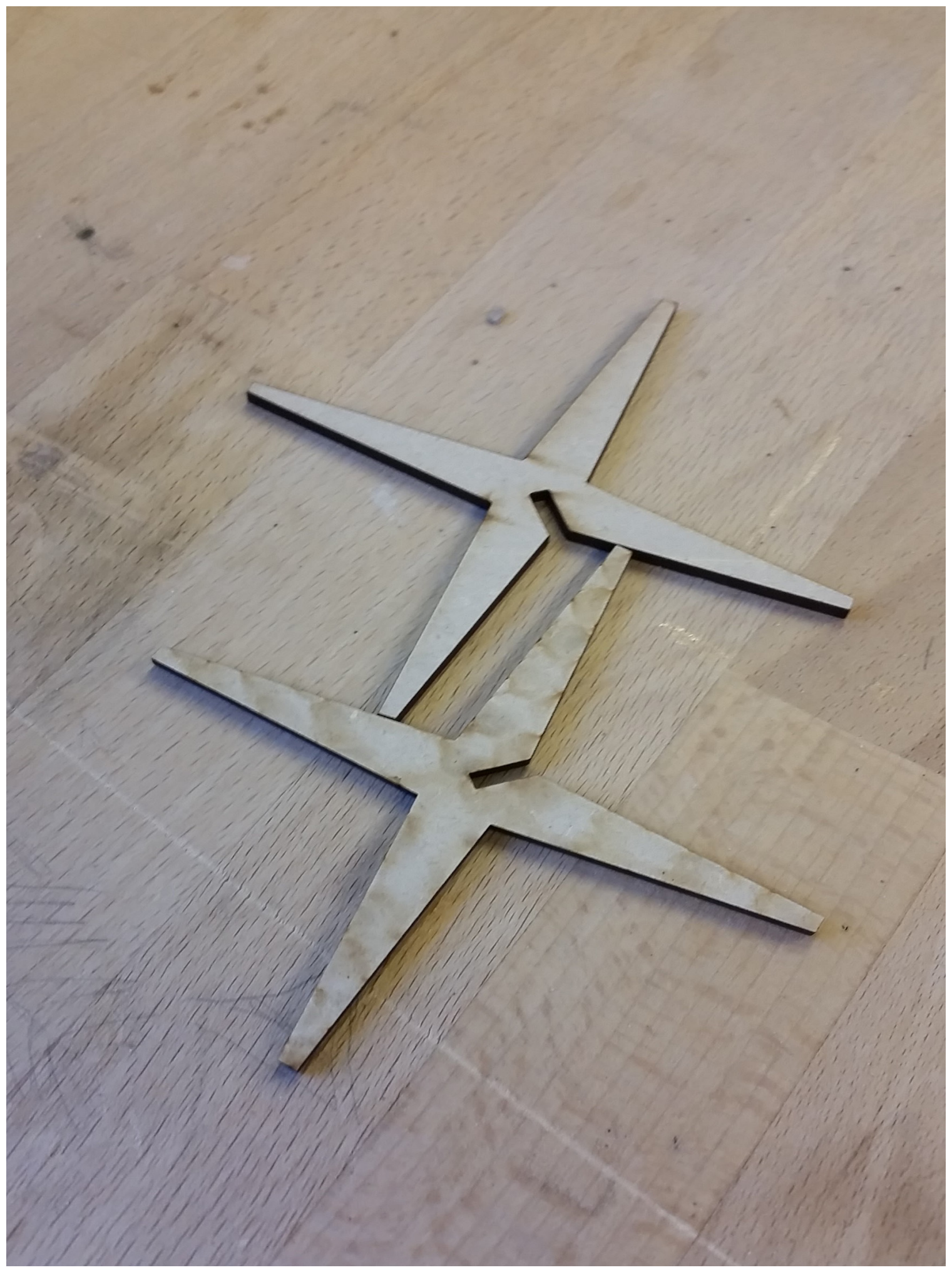

Figure 15. The common design principle of the particles tested consist of two identical plates with slot joints 


\section{Particle Morphology}

A number of different particle variations were tested and its aggregation behaviours compared against the base module (Figure 16) to determine how geometry and material affect stability and malleability. Observations were confirmed quantitatively through published research. The objective was not to provide an exhaustive catalogue of the possibilities or determine the best morphology, but instead to identify some of the relevant factors that affect aggregation and its formal consequences and highlight the relationship between the local and global system and the ability for matter to be programmed.

\section{Experimental set-up}

Based on established methods of testing granular material in external disciplines such as geology and industrial processing, experimental procedures have been adapted for this burgeoning area of research in architecture (Dierichs \& Menges, 2012a). To test stability, a cylindrical column composed of the particle under investigation is formed using rigid building form tubes and mailing tubes. Particles are randomly deposited from a constant height into the hollow tube which is then removed from the top once the desired height is achieved.

One characteristic aspect of the designed granular systems mentioned is their potential to achieve a 90 degree angle of repose unlike most natural granular materials (Figure 17). Stability is determined by observing whether a column can be formed and/or the number of particles that fall off the pile. In some cases, columns that were stable under self-weight were loaded incrementally using one pound bags of rice to structural collapse.

The diameter of the columns are set by tube standards: $127 \mathrm{~mm}\left(5\right.$ "), $203 \mathrm{~mm}\left(8^{\prime \prime}\right)$, and $254 \mathrm{~mm}$ $(10$ "). Variations of the base particle all maintain the star-shaped geometry and the two-plate slot detail (Figure 18). The differences in weight posed by the variations are not accounted for and are assumed to be negligible. 


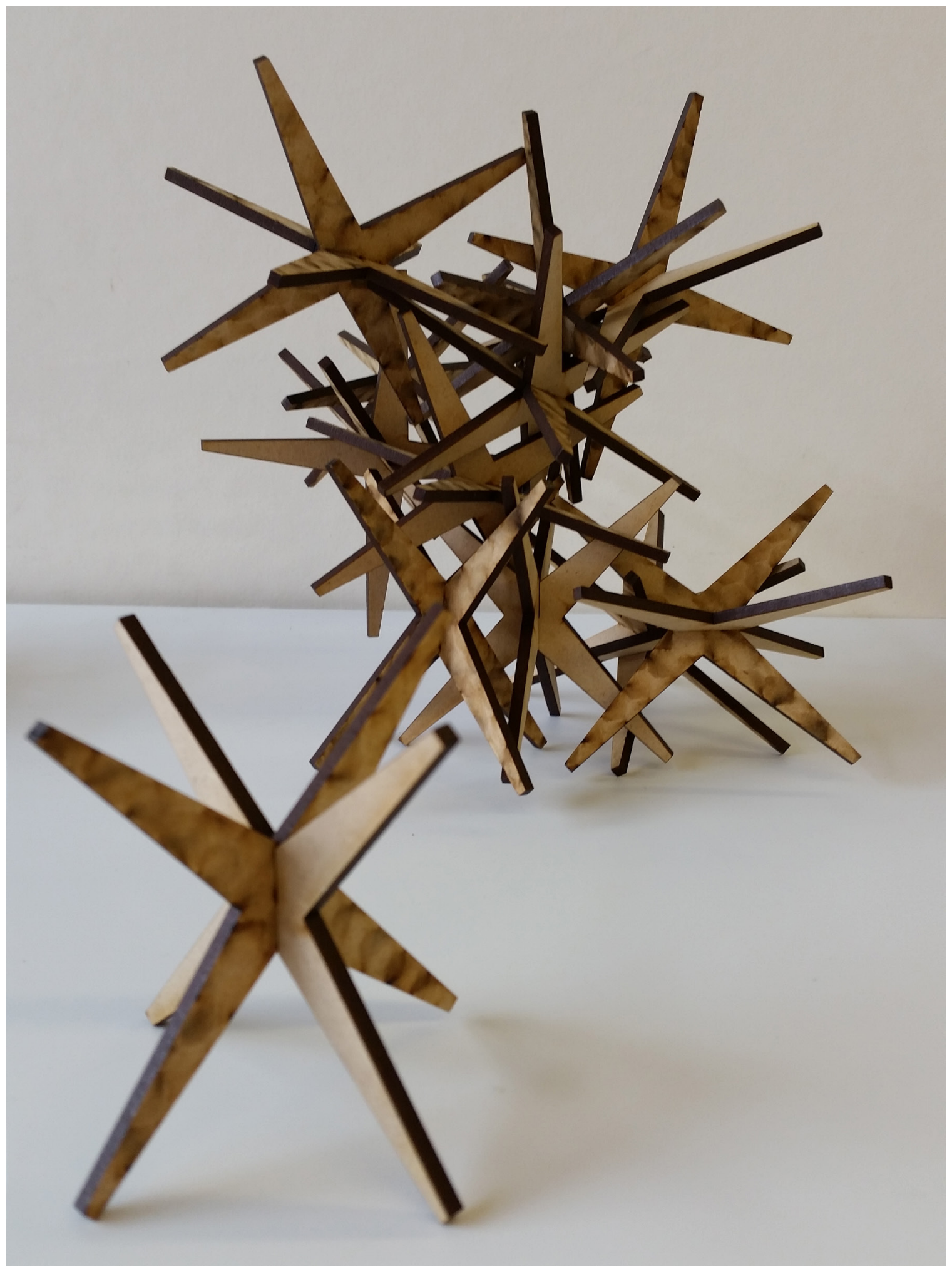

Figure 16. Designed granular display unique aggregation properties. Base particle shown. 


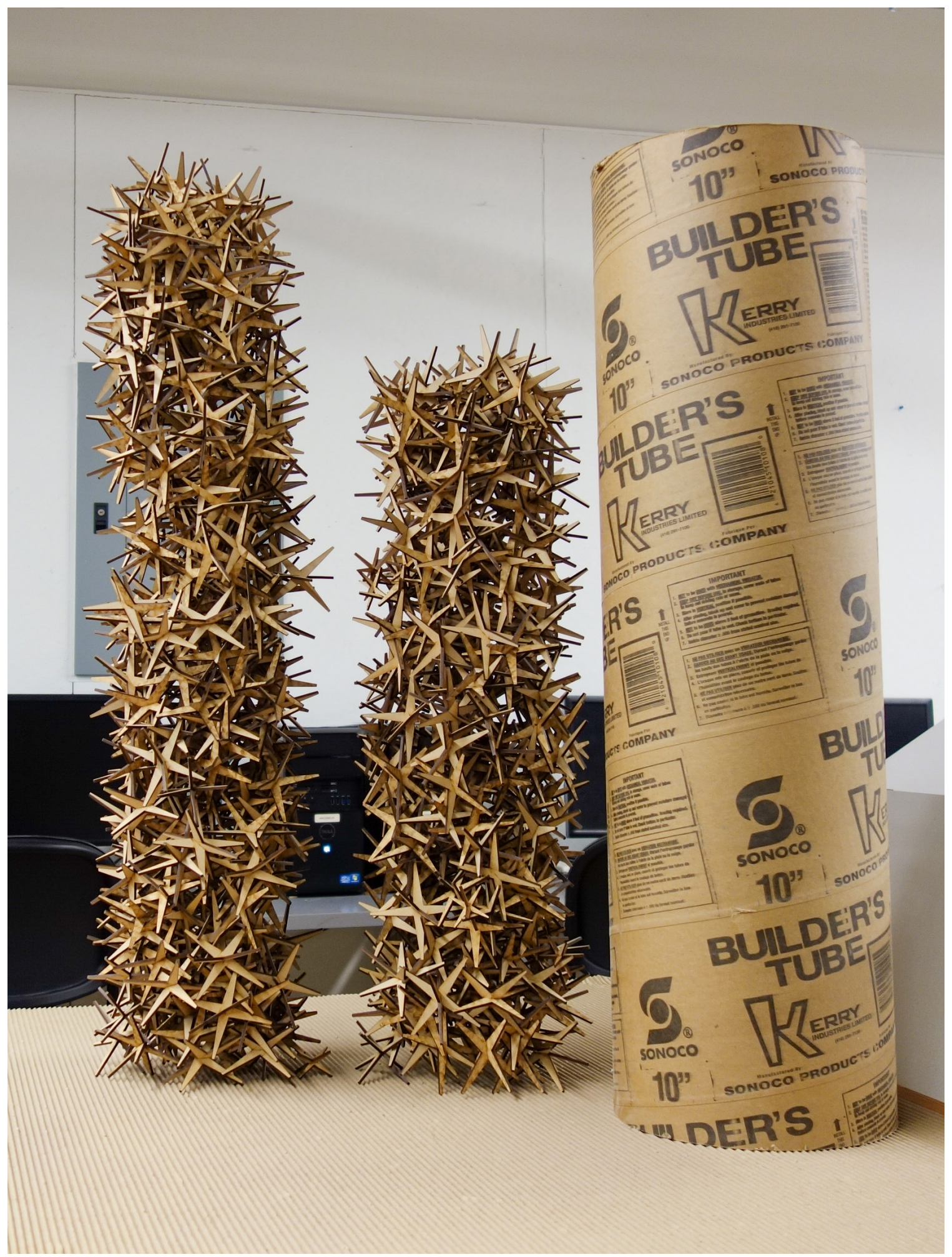

Figure 17. Designed granular materials are able assume columnar forms with a vertical angle of repose

46 Architecture at the Edge of Chaos 


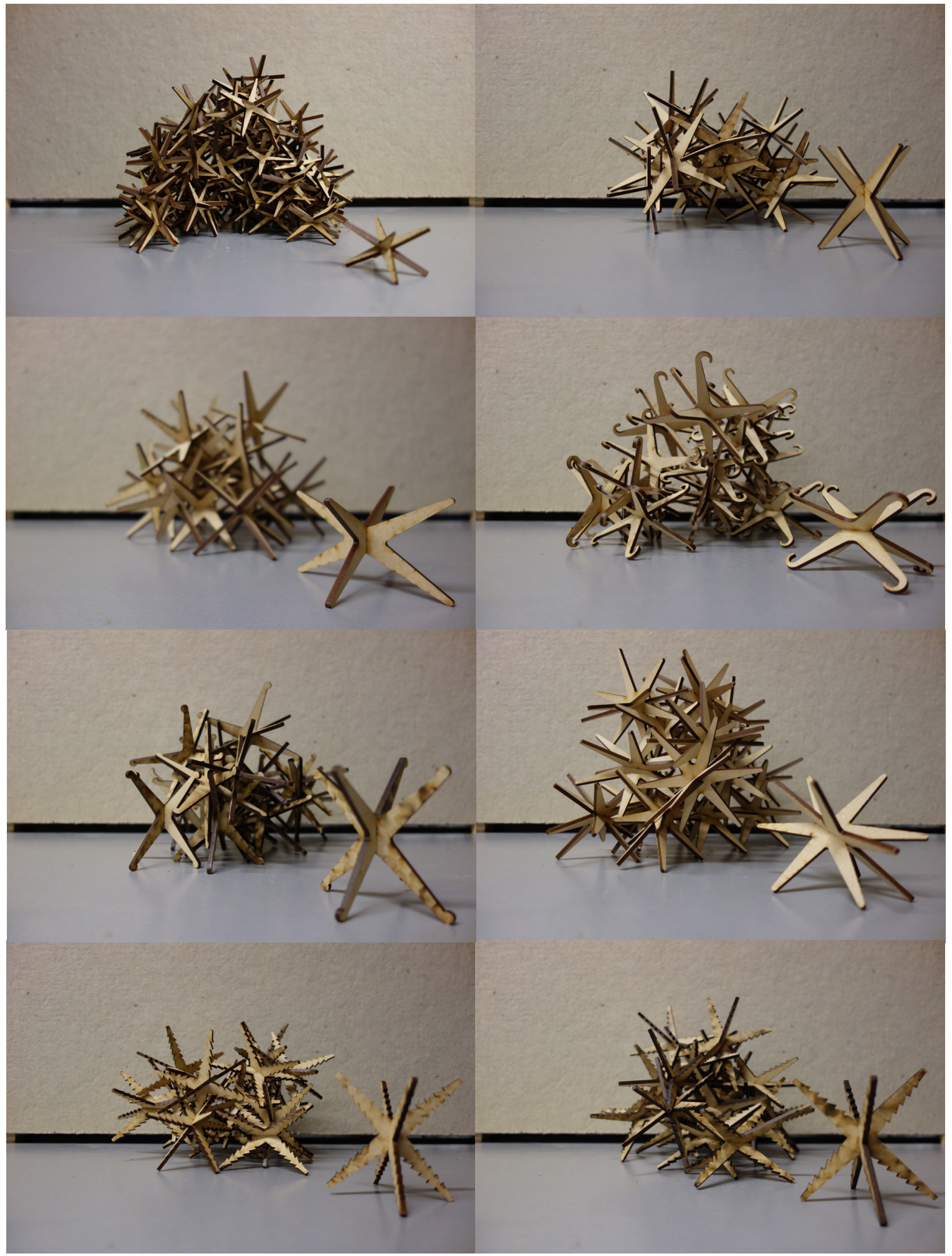

Figure 18. Selection of various particle morphologies 


\section{Base Condition}

The base condition or friction of the base is an important factor in determining overall stability (Zhao et al., 2016). A stable first layer or foundation serves to stabilize the rest of the structure, but this is a significant consideration when the lines of forces have multiple paths which are not particularly clear in such a system. This was most apparent with the large units where the greater weight allows the forces and effects to be more easily perceived. With irregular forms that were created and situated on tile for example, non-vertical component forces can cause the base to slip causing collapse. This was especially evident in the attempts to create vaulted structures on smooth surfaces such as an MDF sheet where the base will tend to thrust outwards without any abutments. As a result, other base materials that provided more friction was tested, including corrugated mats of cardboard, rubber and a bed of sand (Figure 19).

Table 1. Effect of the base condition on pile stability

\begin{tabular}{|c|c|}
\hline Base Condition - Friction & Pile Stability \\
\hline High & High \\
\hline Low & Low \\
\hline
\end{tabular}

It can be argued that the system excels and is best suited for harsh, rough and uneven terrain. Due to its granular make up, differences in levels and can be easily and precisely bridged by modulating the amount and size of the granule.

The subsequent column tests were conducted on an acrylic sheet base. The low friction base creates a sensitive condition to better detect instabilities. 


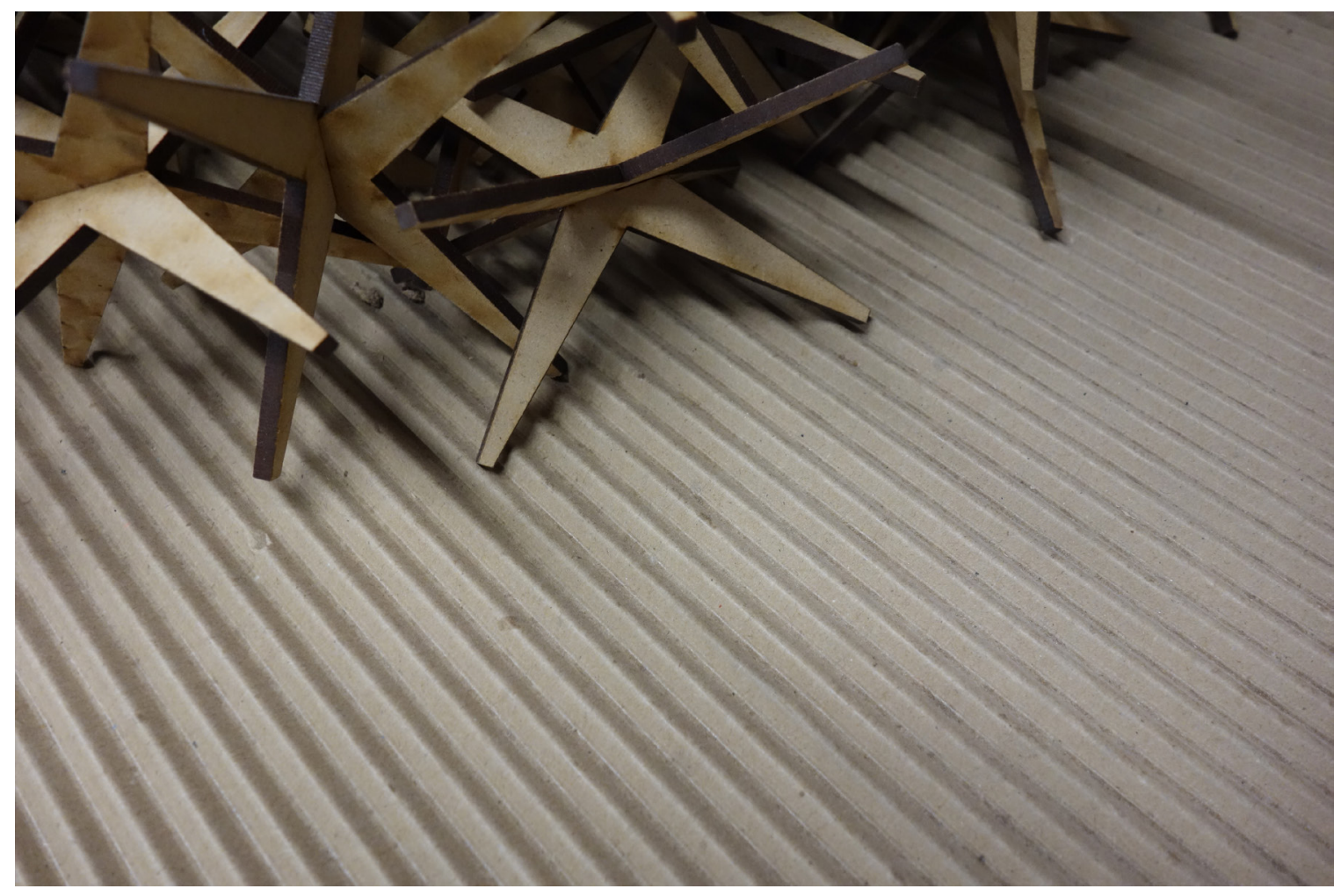

Figure 19. Base condition with corrugated cardboard mat 


\section{Vibration}

The effect of vibration was tested with columns constructed with an 8 " diameter tube using 100 standard particles forming a height of about $50-55 \mathrm{~cm}$. Vibration was initiated by shaking the tube periodically as the particle were deposited. Vibration was found to have a large impact on stability. Vibrated columns were consistently (100\%) found to be able to remain standing with little to no particles falling from the column, whereas non-vibrated columns only remained standing four out of ten times. Vibration appears to allow particles to pack closer together, decreasing the distance between particles, increasing friction between particles and thereby increasing stability (Figure 20). Non-vibrated situations tend to allow "holes" in the structure by exacerbating any uneven distribution and creating weak connections (Figure 21). The subsequent tests were conducted with vibration. The conclusions agree with those from Zhao et al. where more controlled vibrational experiments were conducted with a motor driven eccentric (2016).

Table 2. Effect of vibration on pile stability for columns of $d=8 "$ and $h=50 \mathrm{~cm}$ for base particle

\begin{tabular}{|c|c|c|}
\hline Vibration & Stable Columns (\%) & Pile Stability \\
\hline Yes & 100 & High \\
\hline No & 40 & Low \\
\hline
\end{tabular}




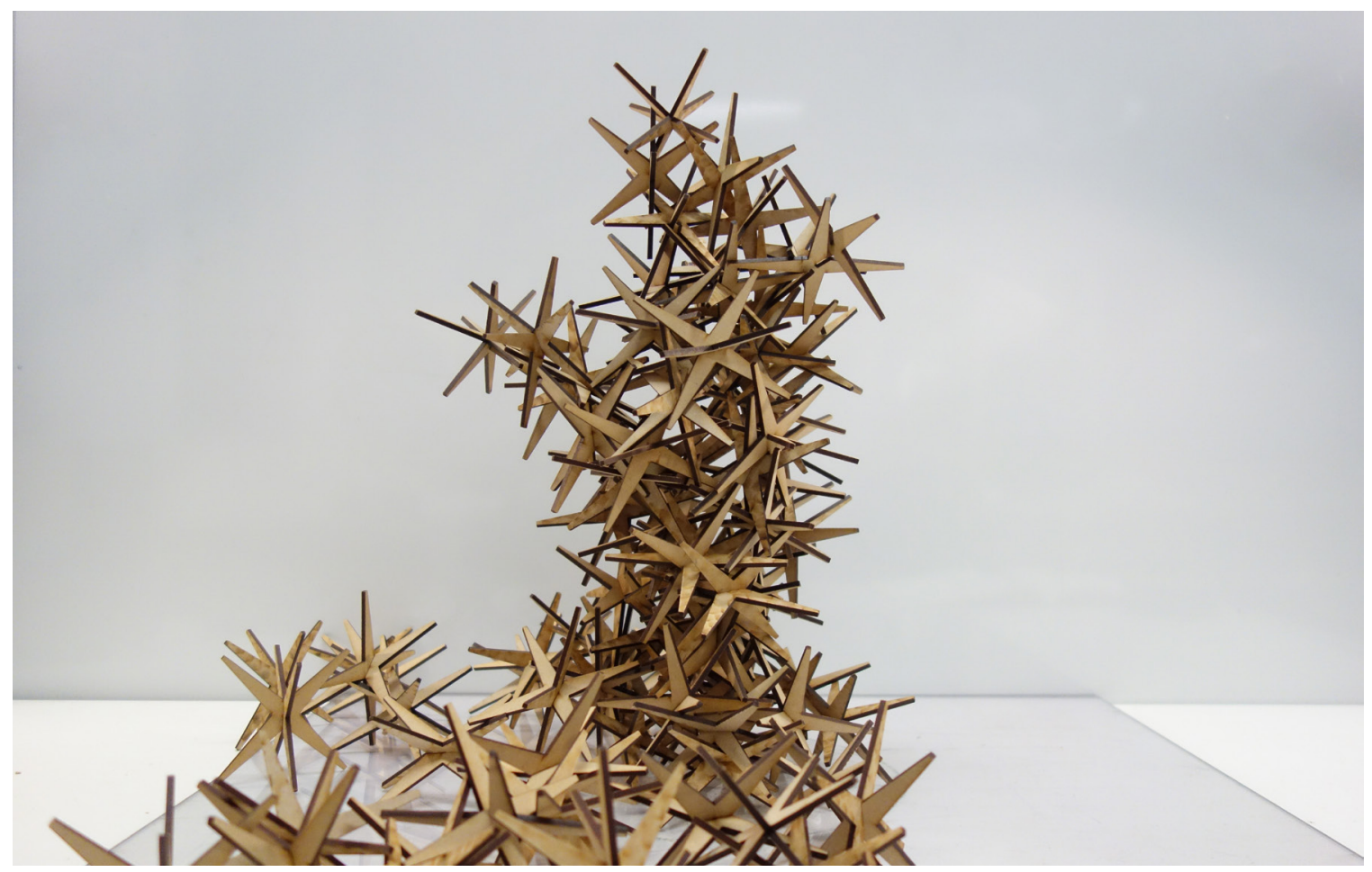

Figure 20. Vibrated column

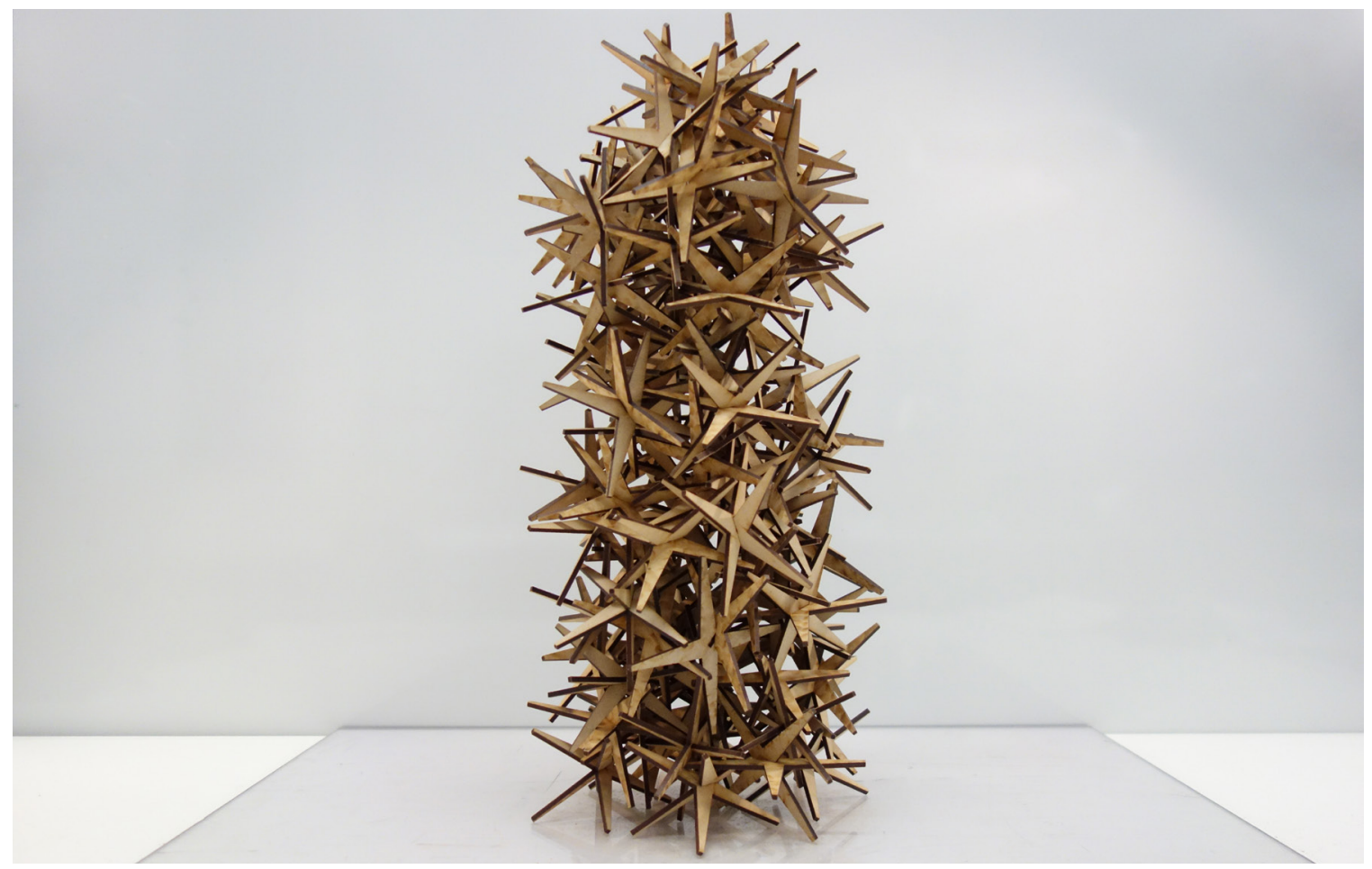

Figure 21. Non-vibrated column 


\section{Arm Length \& Scale}

The base module is compared with two size variables: arm length and scale. The first particle is exactly half the scale (except for material thickness) and the second has truncated arms half the length of the base module (Figure 23). Due to the fact that the base particle arms tapered towards the end, truncating the arms therefore produced wider tips. First, the base and half-scaled particles exhibit identical properties of porosity and cohesion. The height and diameter of the column had been scaled accordingly to provide a relative comparison.

On the other hand the truncated particle was unable to form a column at all (Figure 24). Keeping the core and the centre of the particle identical to the base module, as arm length is reduced relative to its centre, the particle approaches a convex form. In a limited scenario, a medium-length, truncated particle was briefly examined (Figure 25). The medium length particle was a $25 \%$ reduction of the base particle. As expected it exhibited behaviour in between the two extremes conditions tested. Arm length affects packing density and although it is able to pack closer together, diminishing the arms significantly decreases the system's ability to entangle and stabilize. Therefore there is a sharp distinction between scale and arm length even though the effective size may be the same. Longer-armed particles increase stability except however, when the particles size become comparable to the column diameter the base of the structure is reduced to too few particles and becomes unstable (Zhao et al., 2016).

Table 3. Effect of arm length on pile stability

\begin{tabular}{|c|c|}
\hline Arm Length & Pile Stability \\
\hline Long (Base Length) & High \\
\hline Medium & Med \\
\hline Short & Low \\
\hline
\end{tabular}

By adjusting the length of the arm, stability of the material system can be controlled. Mixing different particles sizes can be another way to modulate the viscosity (Figure 26 and Figure 27). The truncated particles allowed for greater malleability while the half-scaled particles was found to increase cohesion and stability (see Design Exploration 1).

Table 4. Effect of mixed scaled particles on pile stability

\begin{tabular}{|c|c|c|}
\hline Mixture (200 pc, 50:50) & Stable Columns (\%) & Pile Stability \\
\hline Base + Half-Scaled & 100 & High \\
\hline Base + Truncated & 50 & Low \\
\hline
\end{tabular}




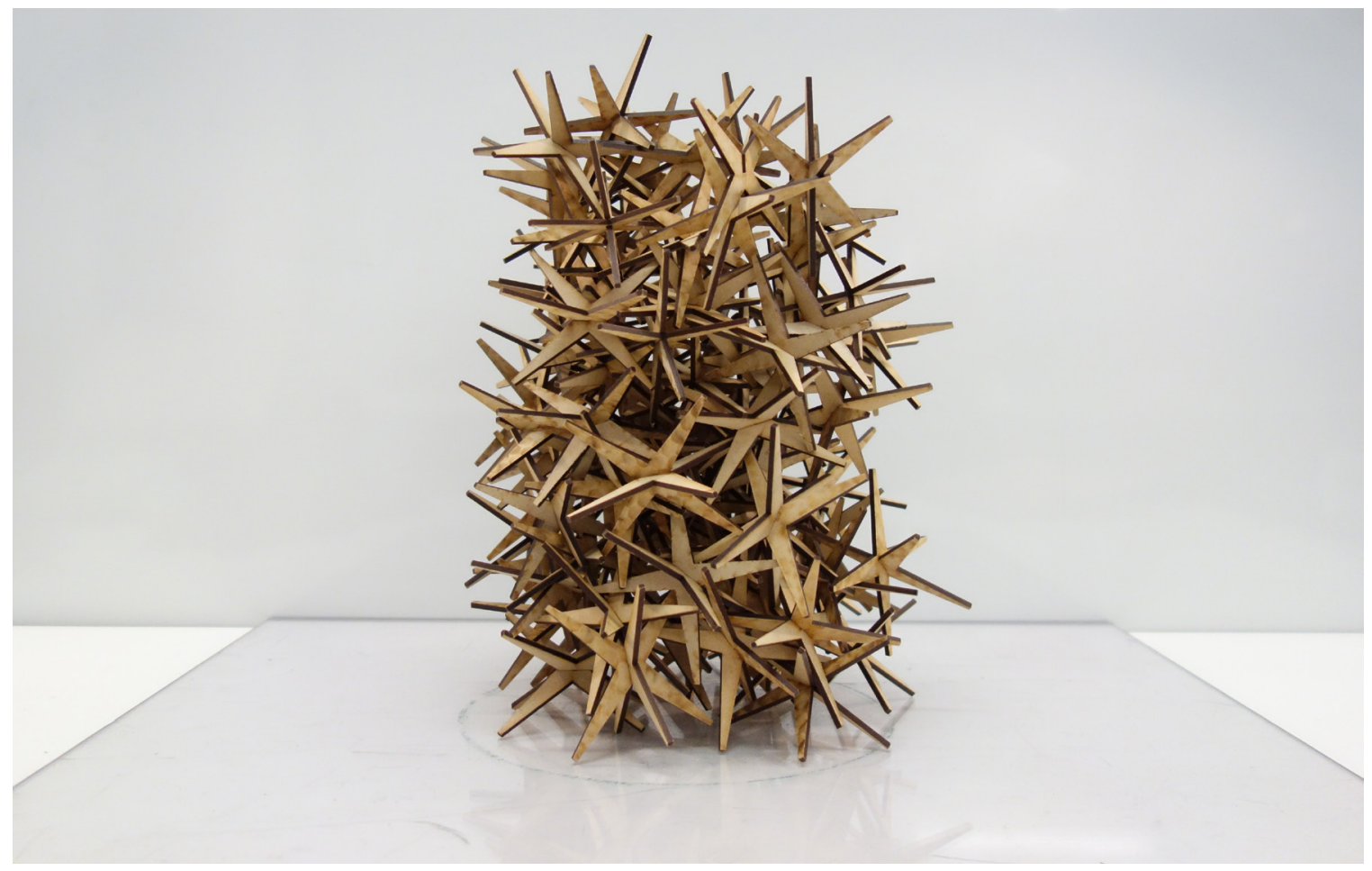

Figure 22. 10" d column of 100 pc, base particles

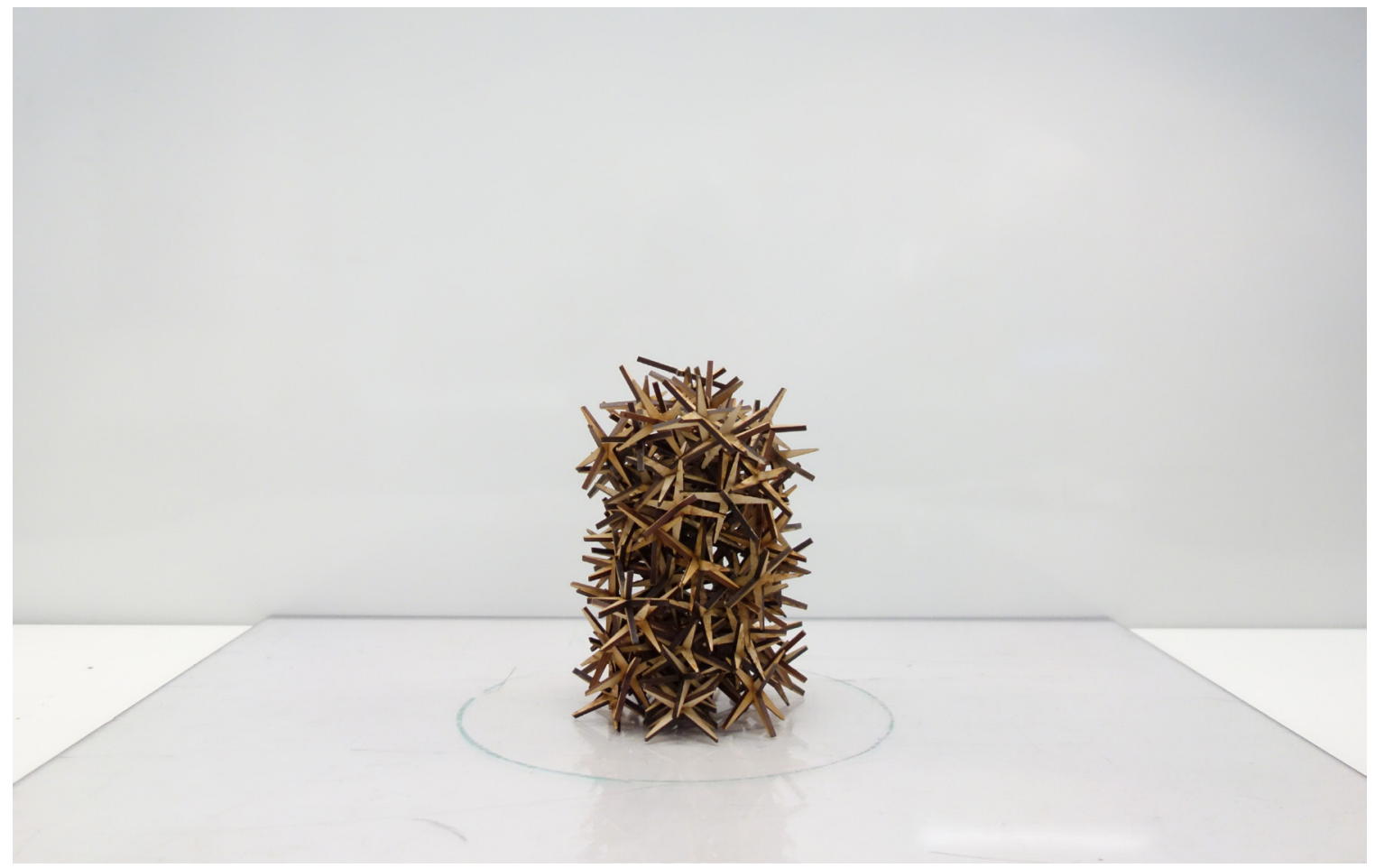

Figure 23. 5" d column of 100 pc, half-scaled particles 


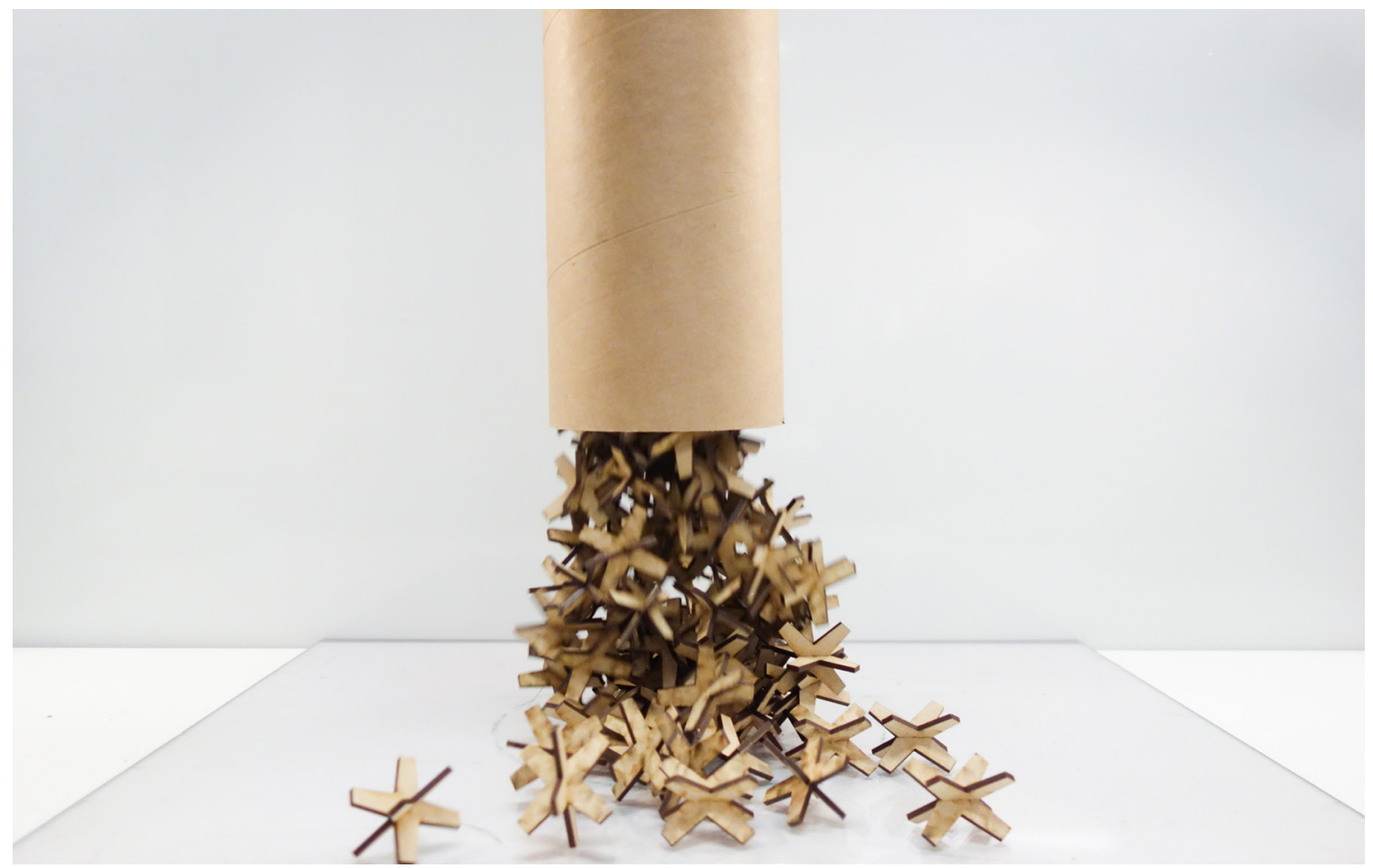

Figure 24. Attempt to form 5" d column of 100 pc, truncated particles

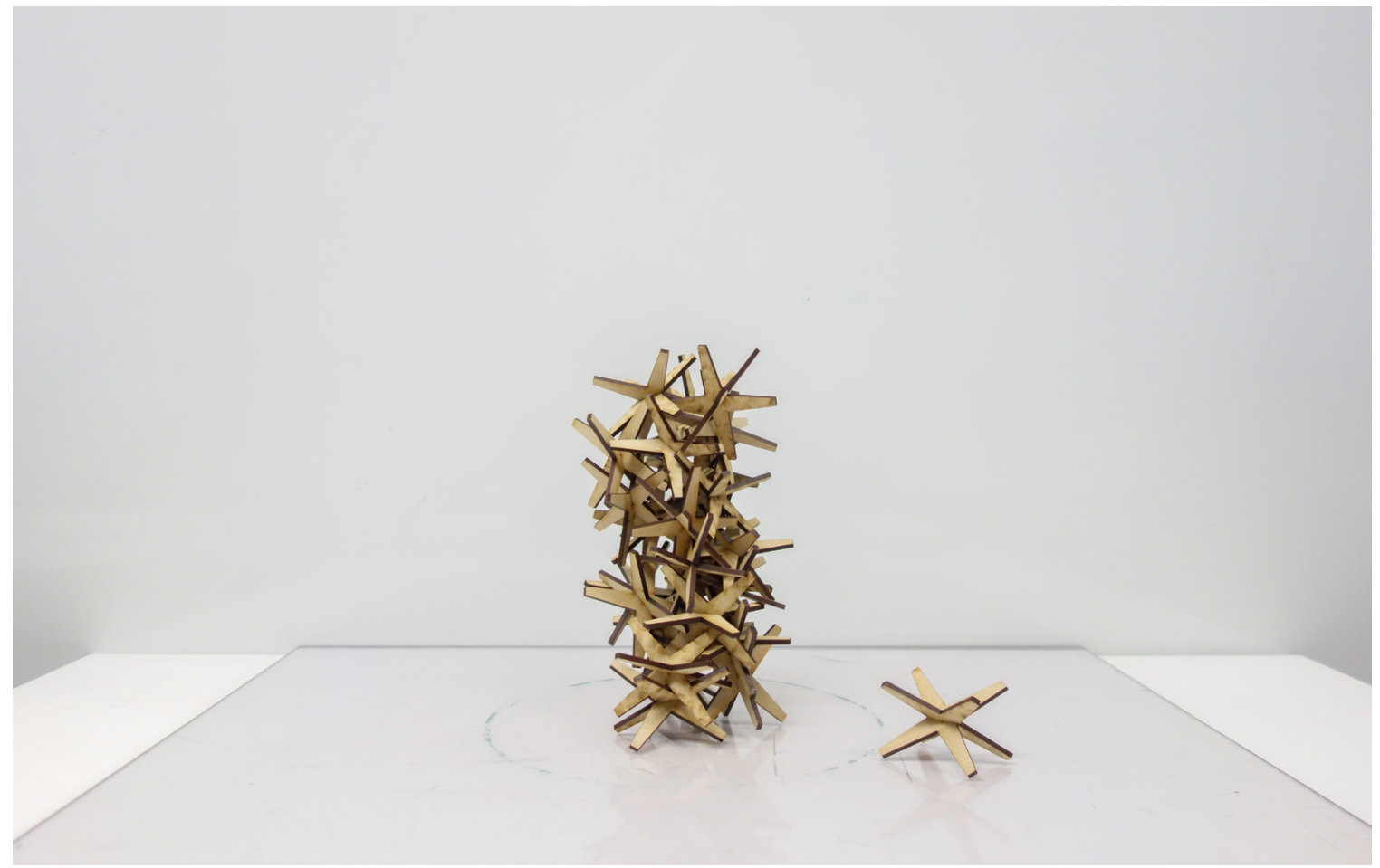

Figure 25. 5" d column of 28 pc, medium-length truncated particles

54 Architecture at the Edge of Chaos 


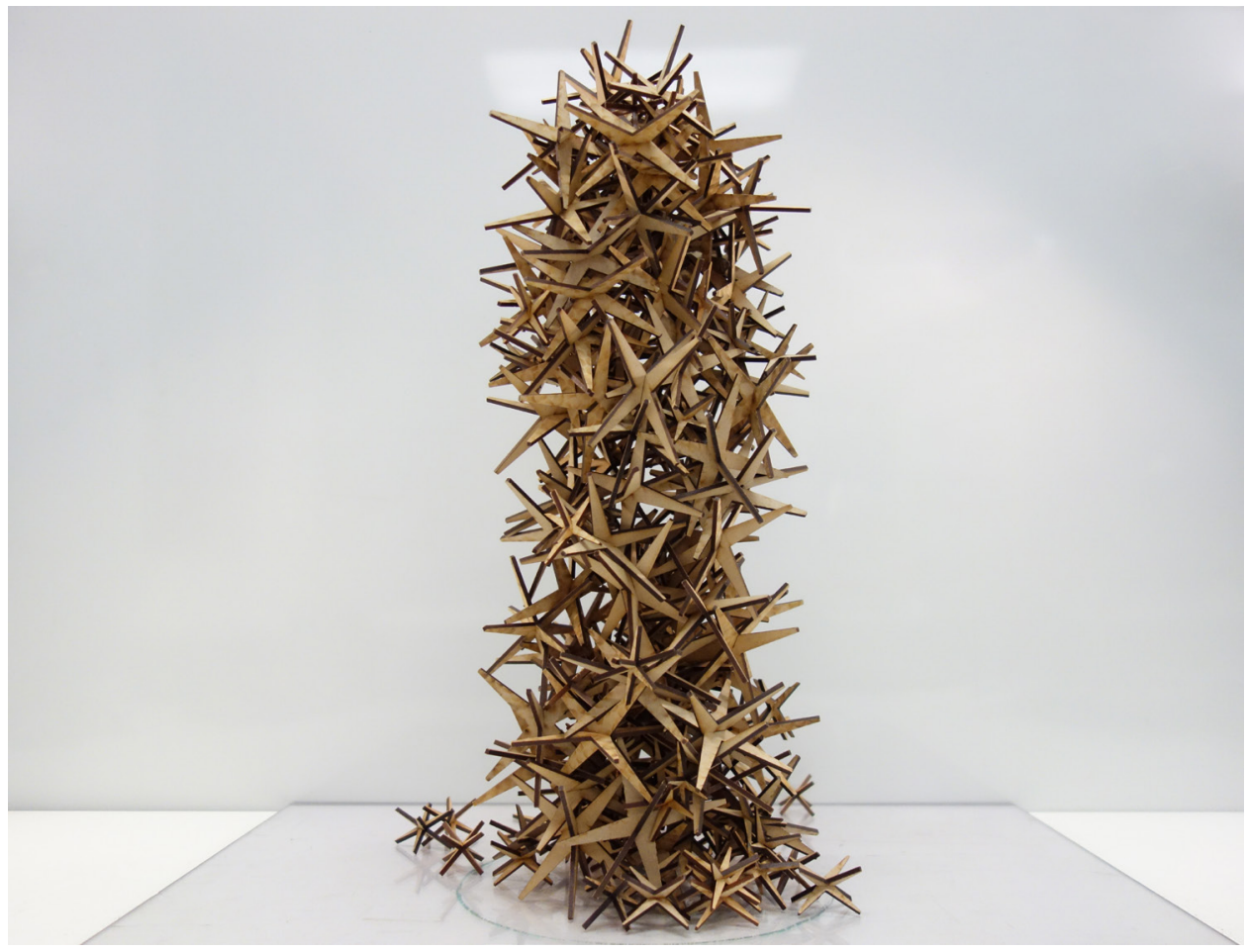

Figure 26. 200 pc equal mixture of base and half-scaled particles

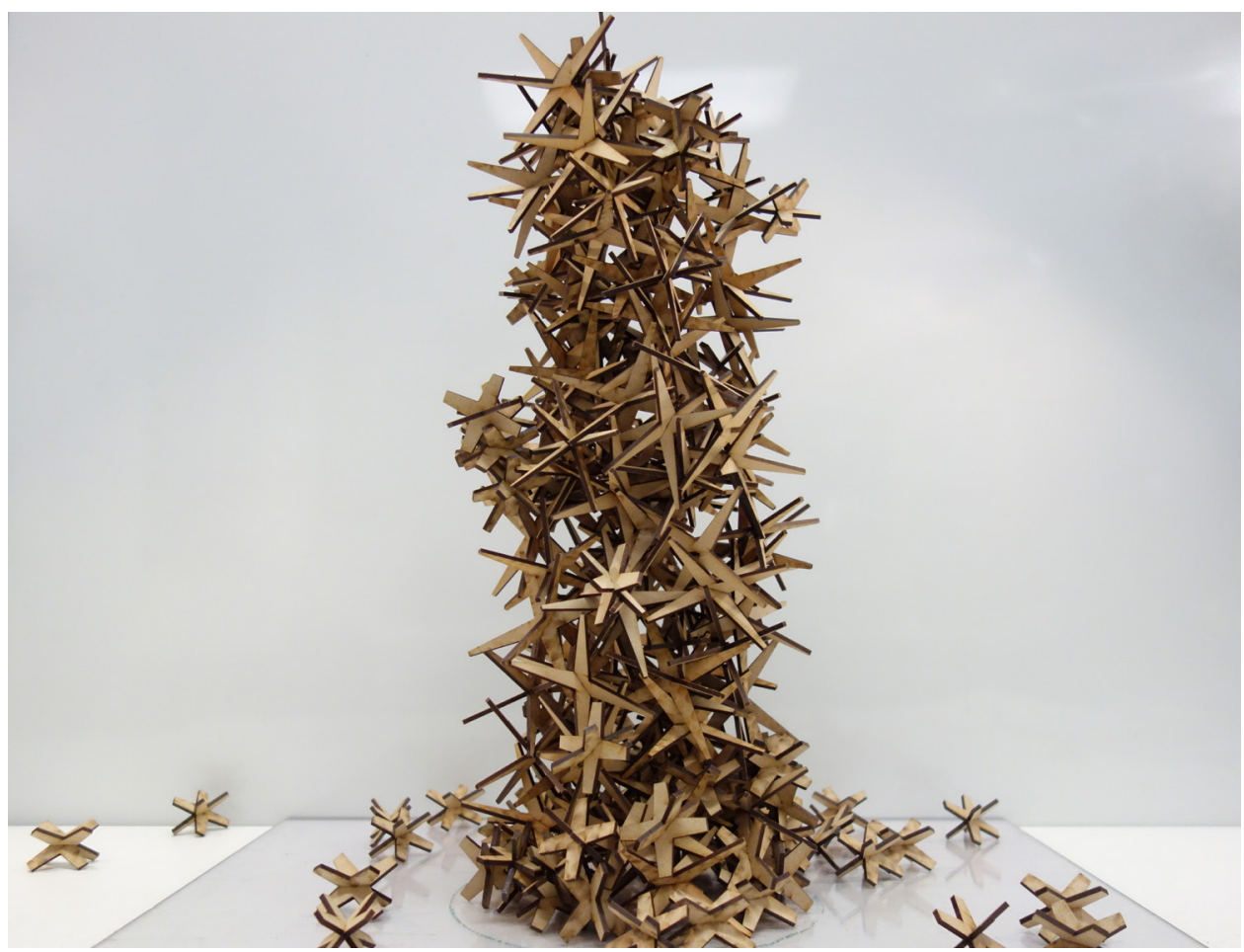

Figure 27. 200 pc equal mixture of base and truncated particles 


\section{Arm Quantity}

Arm quantity per particle also affects packing density. The quantity of arms was controlled by adding or subtracting arms from the component plates, but always keeping the constituent plates identical. 4-arm and 12-arm particles were tested. While it was demonstrated that the arms are vital for entanglement, increasing the number of arms can prevent deep interlocking as can be seen in the case of the 12-arm particle with its greater porosity and larger effective volume (Figures 28, 29, 30). Relative to the 8-arm base particle this results in less stability. Loading similar height columns incrementally with one pound bags of rice, the 12-arm column was only able to support three pounds before collapsing versus the 8 -arm column which was able to withstand four pounds (Figure 31). Collapse usually occurs from an uneven distribution of load due to bag placement, but it can also give way when the weight exceeds what the material composition can bear and the arms start breaking. As a side observation, loading the columns (before collapse) has the effect of forcing greater jamming between particles and making the structure more solid. This explains how one can pick up the column more easily when pressure is applied at the ends (Figure 32). Murphy et al provides a detailed account of pre-straining (2016)

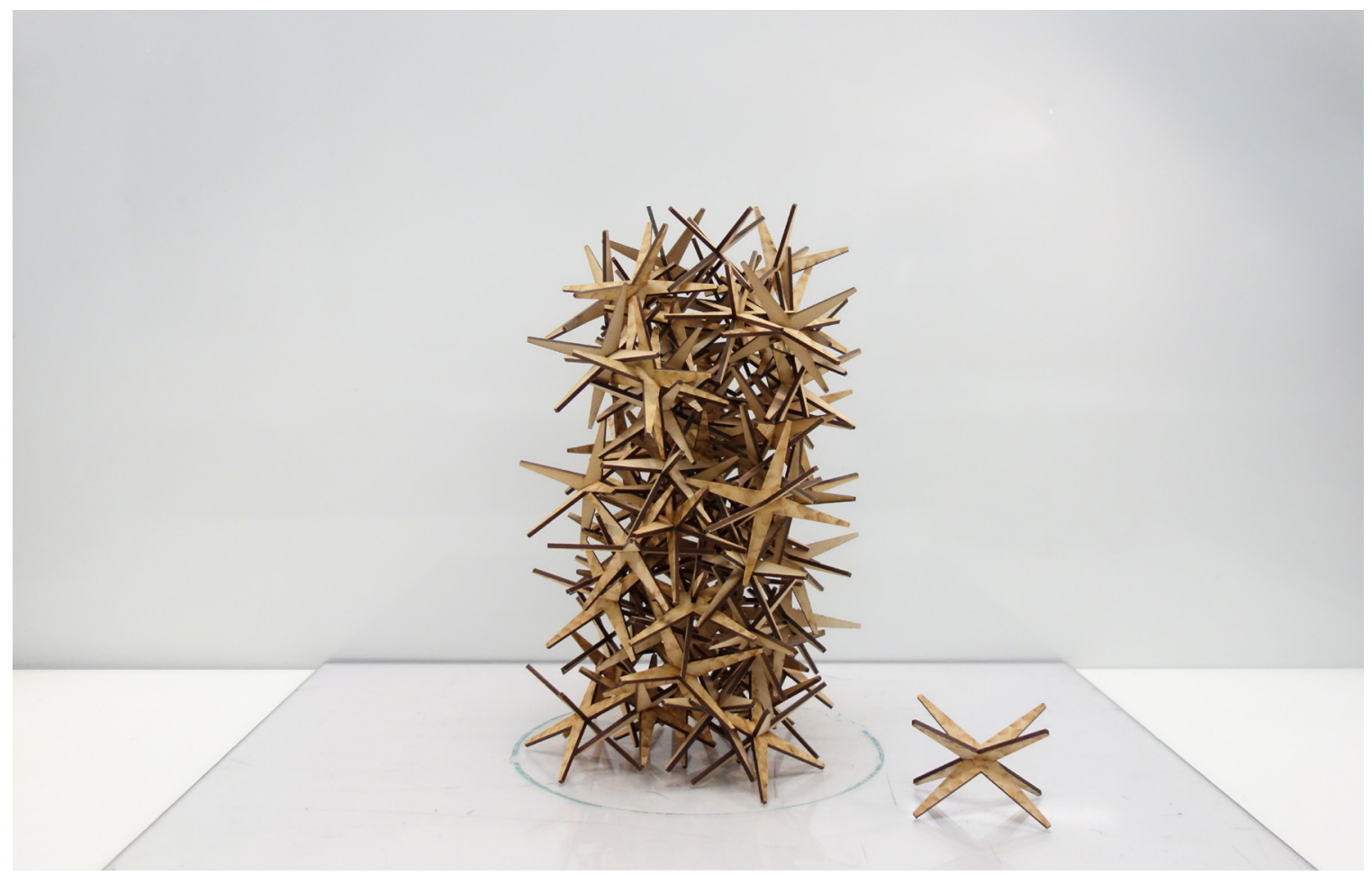

Figure 28. 70 pc base particle 


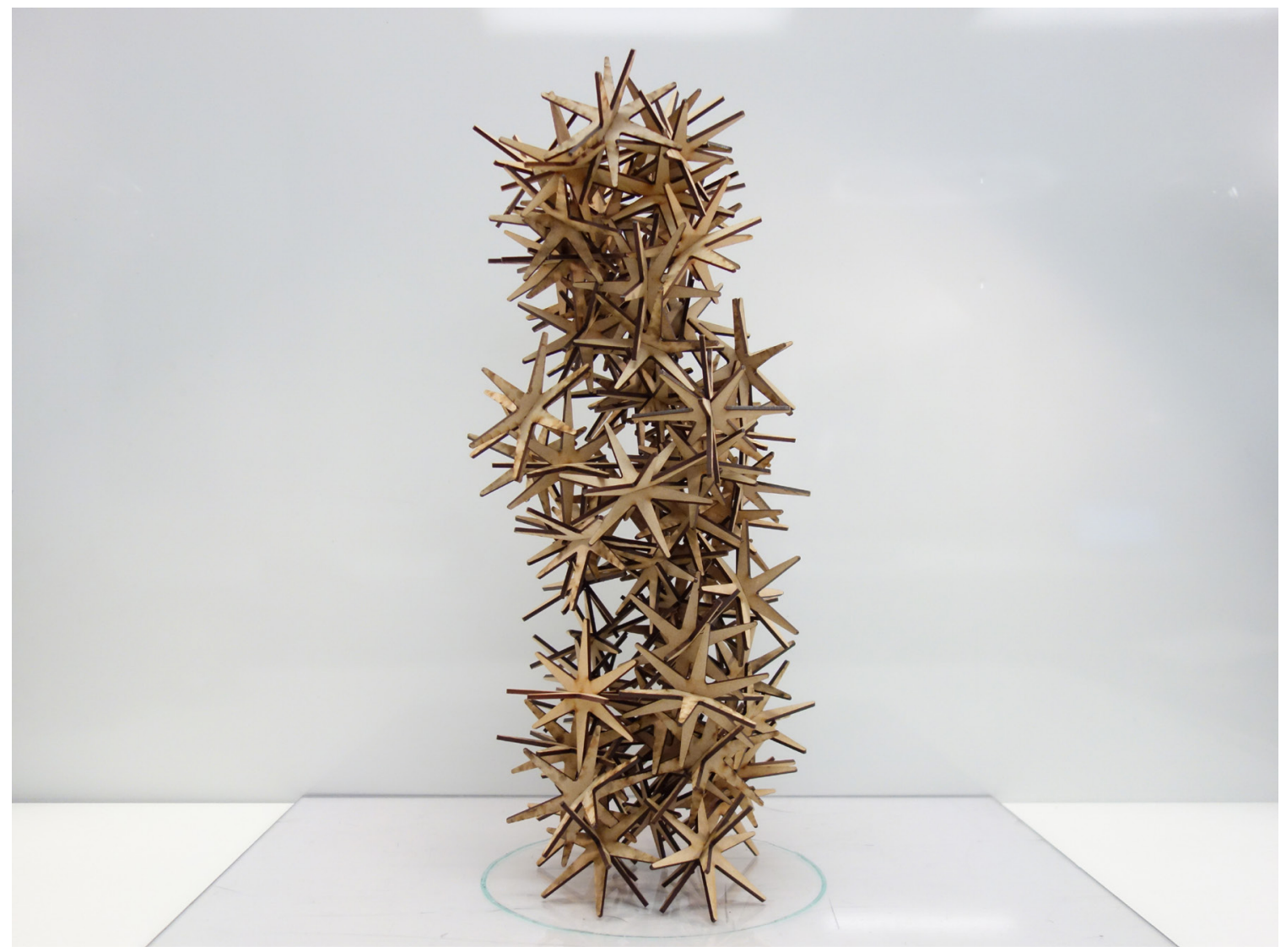

Figure 29. 70 pc 12-arm particle

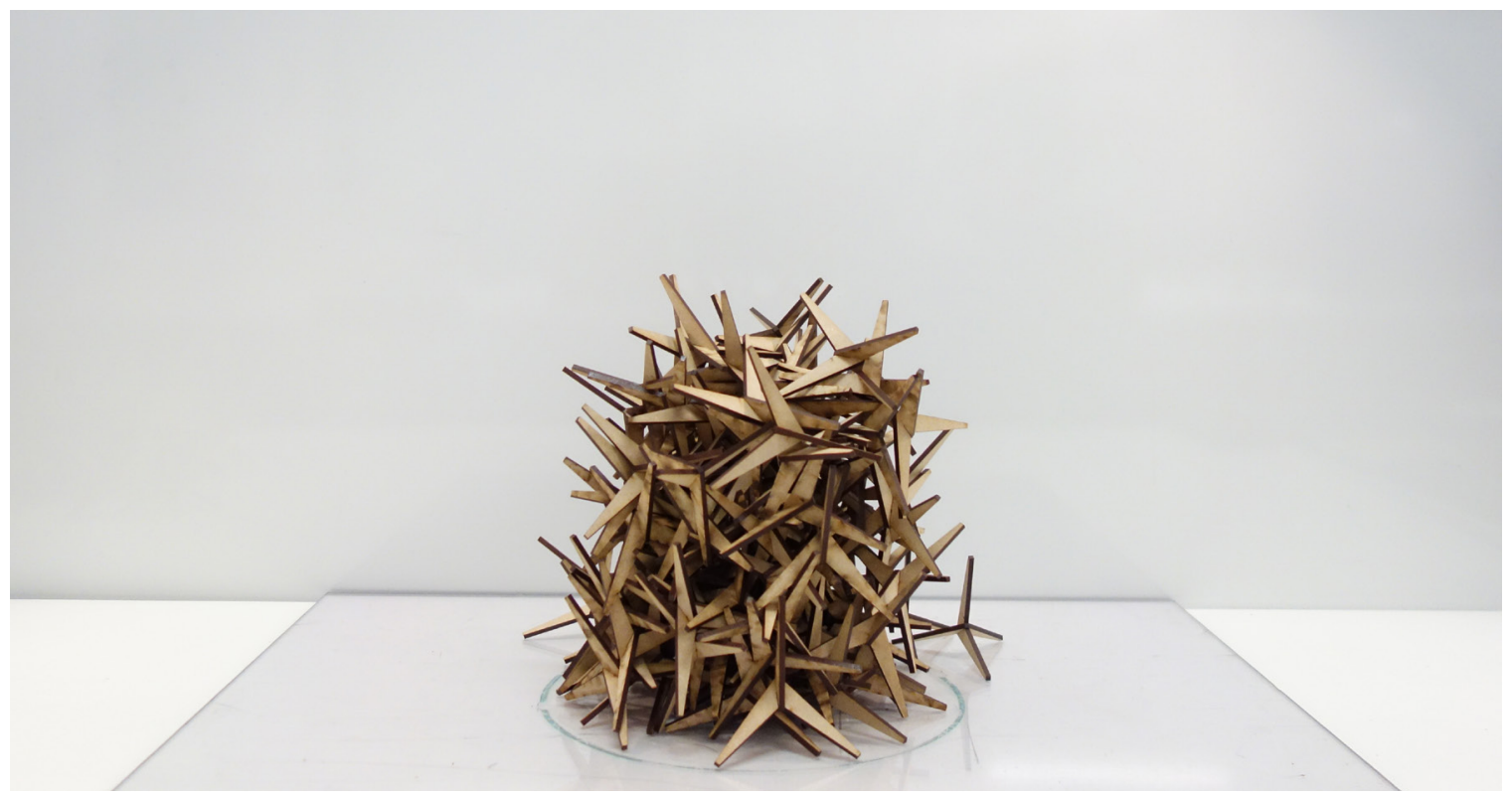

Figure 30. 140 pc 4-arm particle 

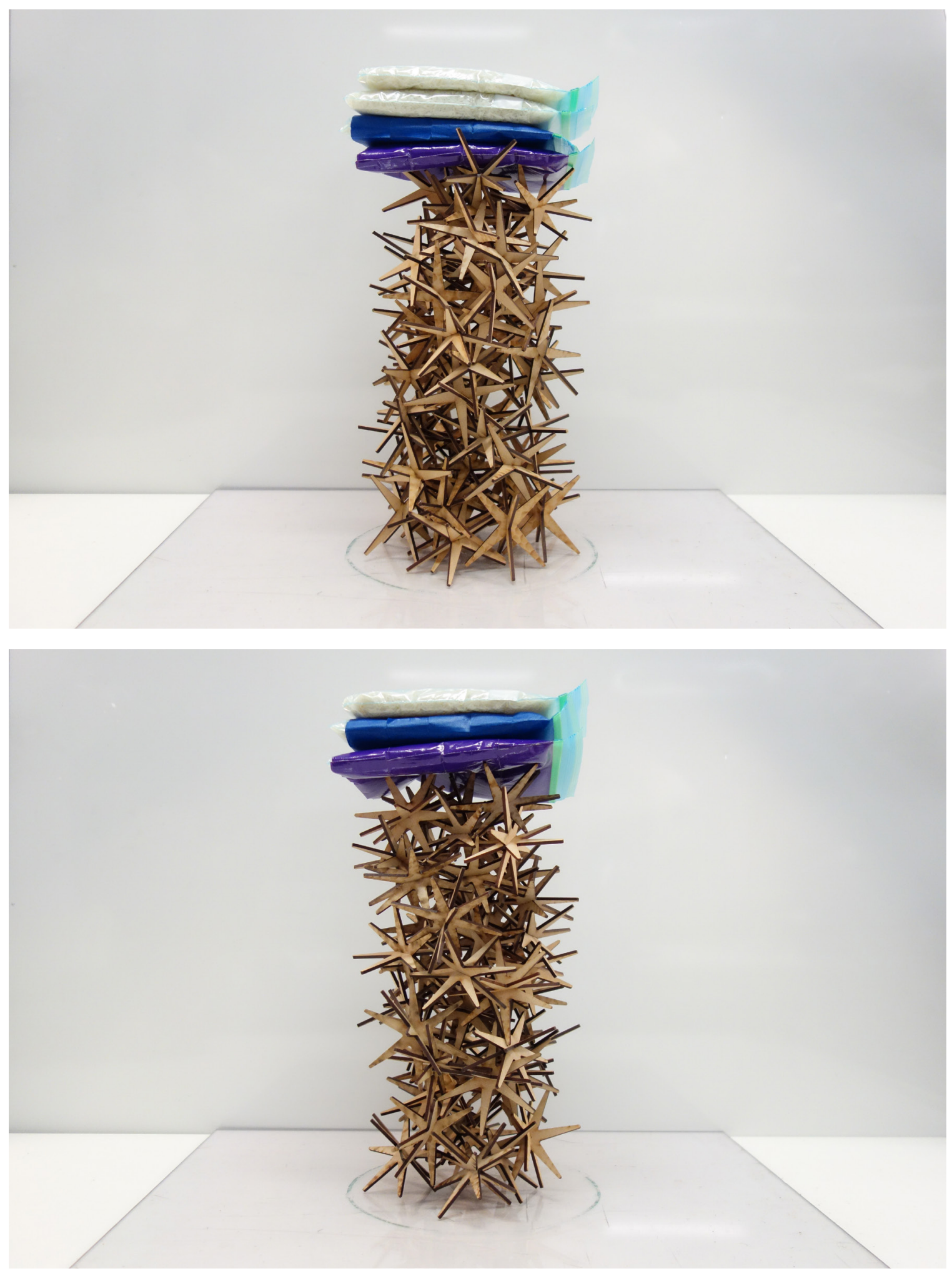

Figure 31. a) 8-arm column (74 pc) supporting 4 pounds; b) 12-arm column (50 pc) supporting 3 pounds

58 Architecture at the Edge of Chaos 
Although the 4-arm particle produces a denser form (Figure 30) its reduced number of arms limited the potential for interlocking. The 8-arm base particle was found to be more stable than both, but the 4-arm particle was the least stable. Compared to the previous truncated particle, the 4-arm particle was more stable. It was found that arm amount affects packing density to a lesser degree than arm length, which Dierichs and Menges also note has relevance for fabrication as arm length is easier to address than geometric complexity (Dierichs \& Menges, 2016).

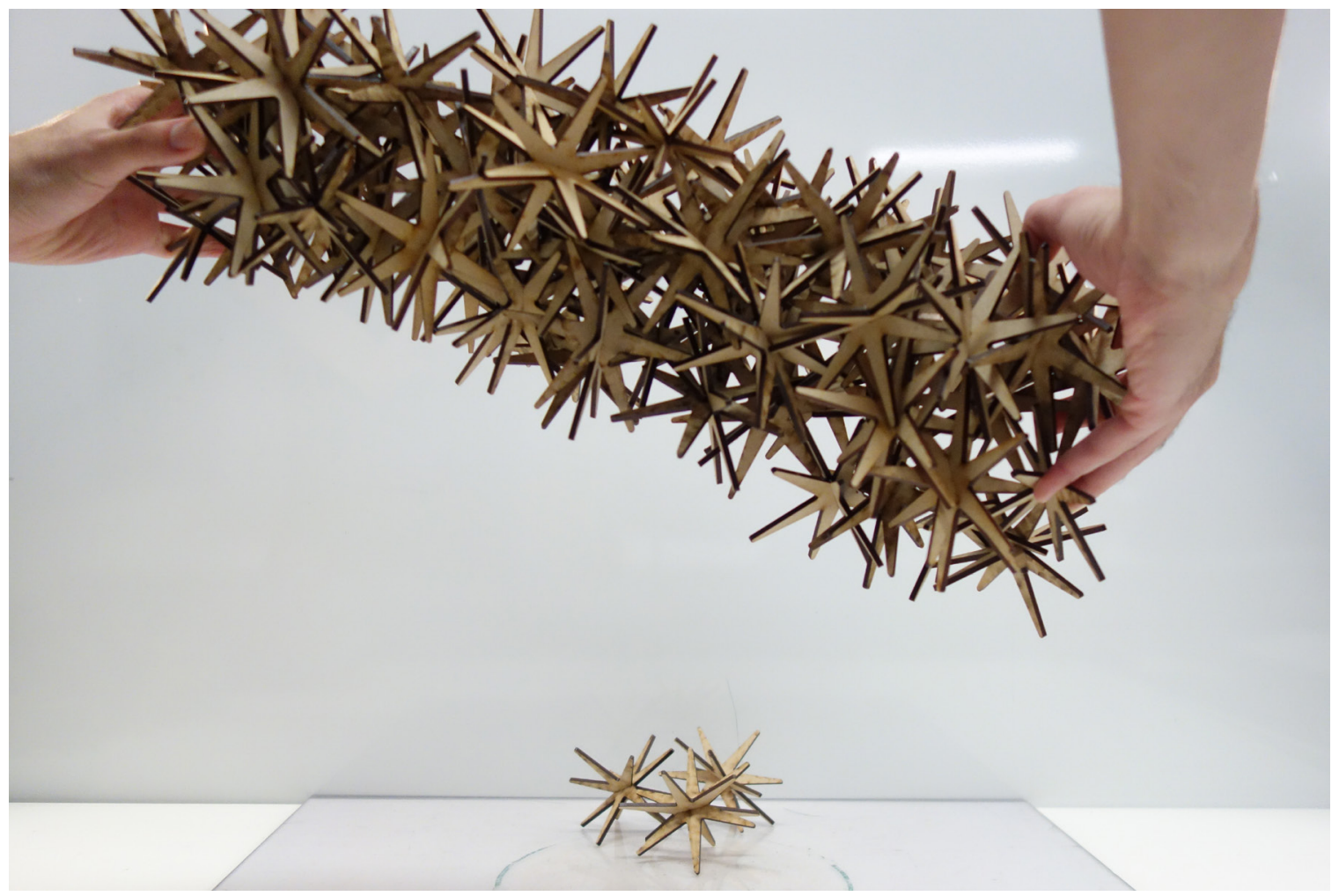

Figure 32. Pre-straining increase rigidity 
It must be acknowledged that as a consequence of the arm quantity, the angle between the arms is also affected and presents another variable to be considered. Changing the angle between arms control the particle's overall aspect ratio and is stated to influence the angle of repose of the pile (Figure 33) (Dierichs \& Menges, 2012b). In the extreme conditions, increasing the number of arms forces the particle to converge to a convex solid, while conversely, decreasing the number of arms can have a similar effect also. Therefore, with arm quantity, a balance must be made.

Table 5. Effect of arm quantity on porosity and pile stability

\begin{tabular}{|c|c|c|}
\hline Number of Arms & Porosity & Pile Stability \\
\hline 4 & Low & Low \\
\hline 8 & Med & High \\
\hline 12 & High & Med \\
\hline
\end{tabular}

As was the case with the arm length variable, mixing particles with different arm quantities can further be employed to program the behaviour of the aggregated mass adjusting structural properties of stability and malleability with architectural considerations such as porosity and permeability (Figure 34). In the mixed column in this case, the different particles were stratified demonstrating another possible combination strategy concentrating specific properties in desired areas (Figure 35). 


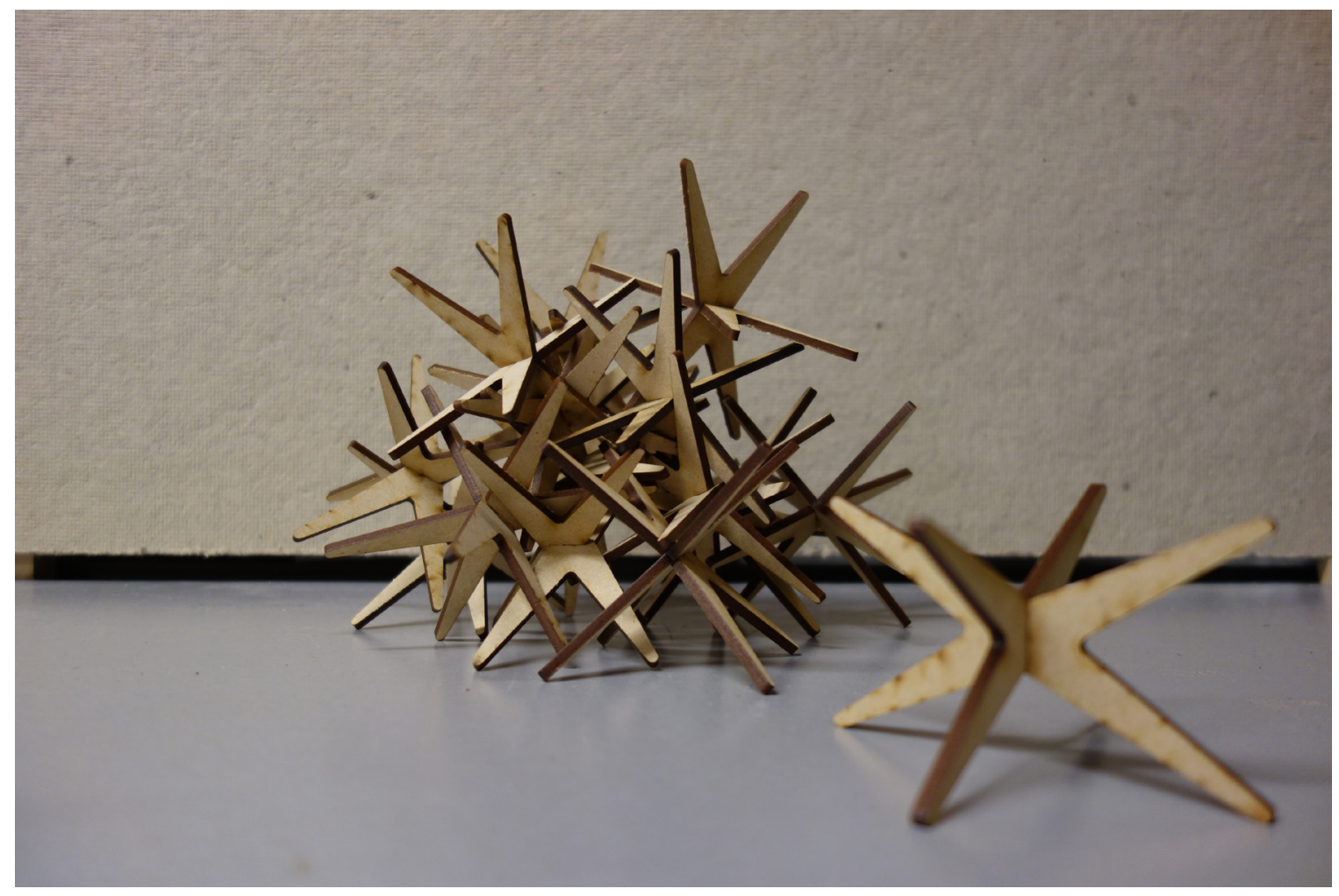

Figure 33. The aspect ratio of the particle is controlled by the angle between arms 


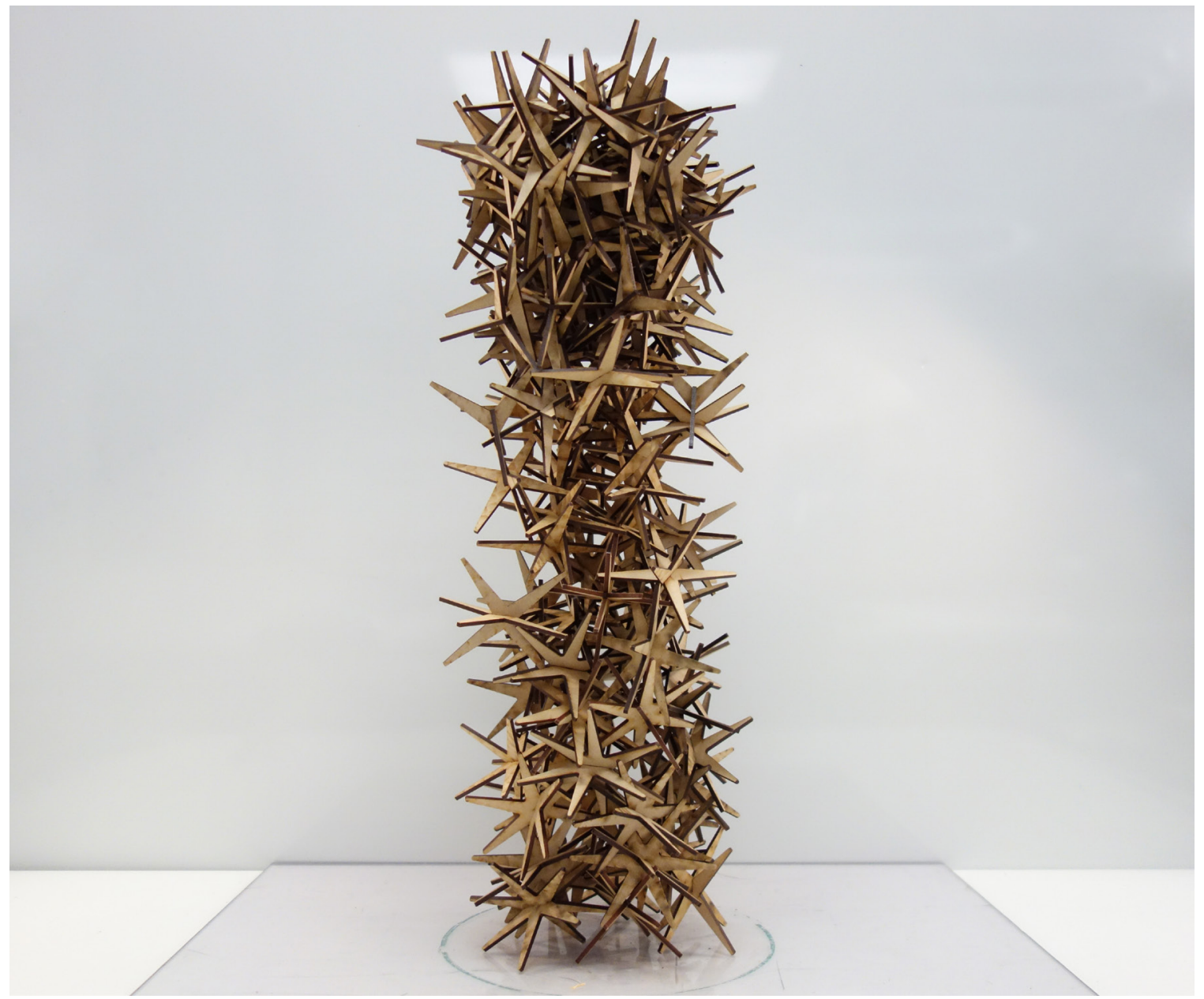

Figure 34. Stratified mixture of particles 

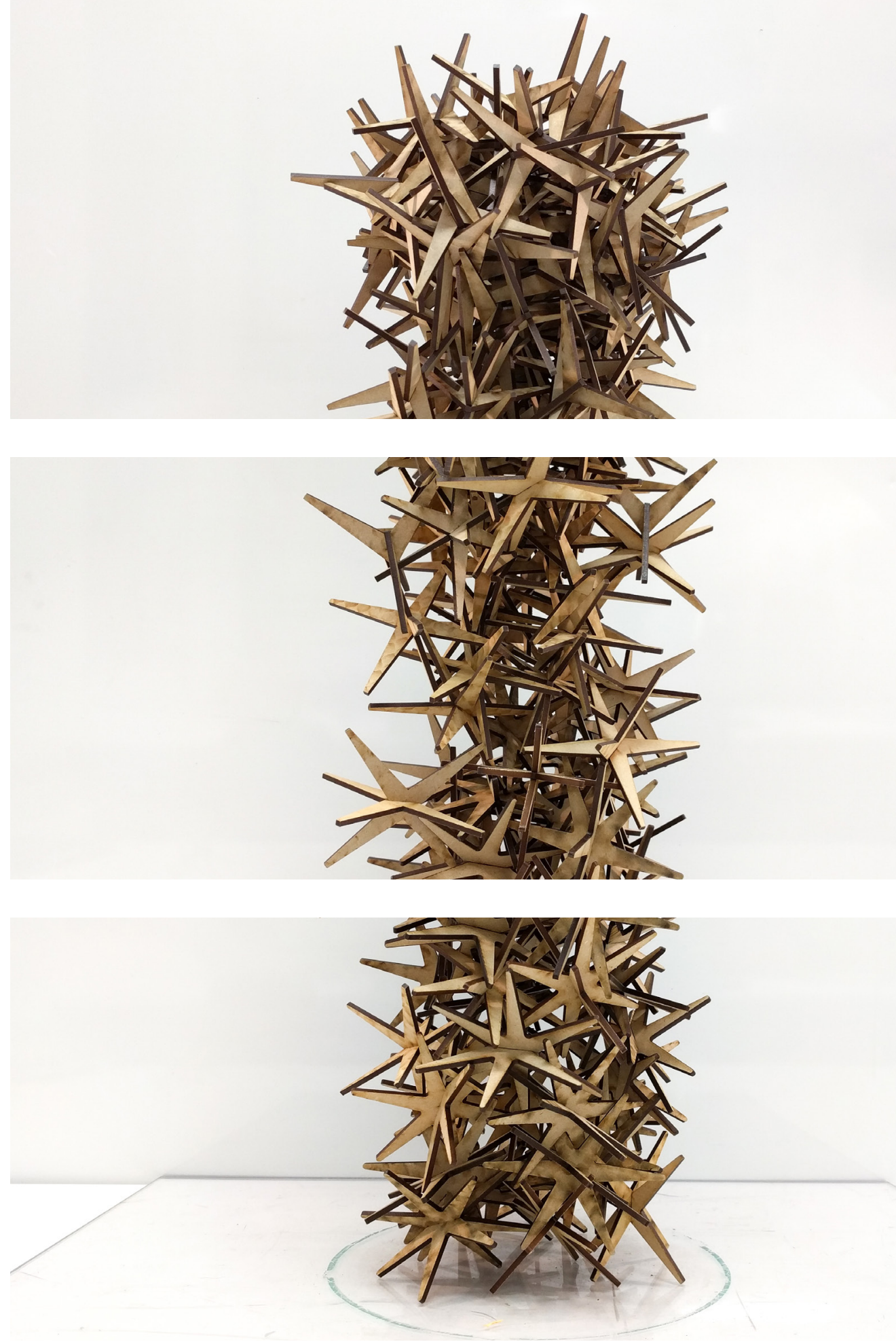

Figure 35. Close up of layers (top to bottom): 4-arm (140pc), 8-arm (35 pc), 12-arm (35 pc) 


\section{Friction (Material)}

Inter-particle friction is key to the performance of an architectural granular material system. The effects of geometry on particle interaction and friction has been demonstrated, but another factor that controls this property is the friction from the material makeup of the particle itself. Smoother, low friction particles were fabricated using acrylic and was observed against high frictional components achieved by coating MDF modules with rubber which produced a somewhat sticky texture (Figure 36 and Figure 37).

The acrylic particle enables freer movement between particles forcing greater reliance on its geometry for jamming. Its aggregate behaviour exhibits greater fluidity and less stability. In fact, since the acrylic components are able to slide past each other with little friction, the slot joints for a particle was prone to come apart; for this reason adhesives had to be used. Also, they were found to be quite brittle and broke easily when dropped. With the rubber coated particles, it was discovered that particles can be held together purely by material friction (Figure 38). In one column test, even as the top of the column appeared to be about to fall off, its ability to hold together was greatly improved by the friction of the material (Figure 39). This afforded forms not possible by the acrylic counterpart, such as greater cantilevers (Figure 40). 


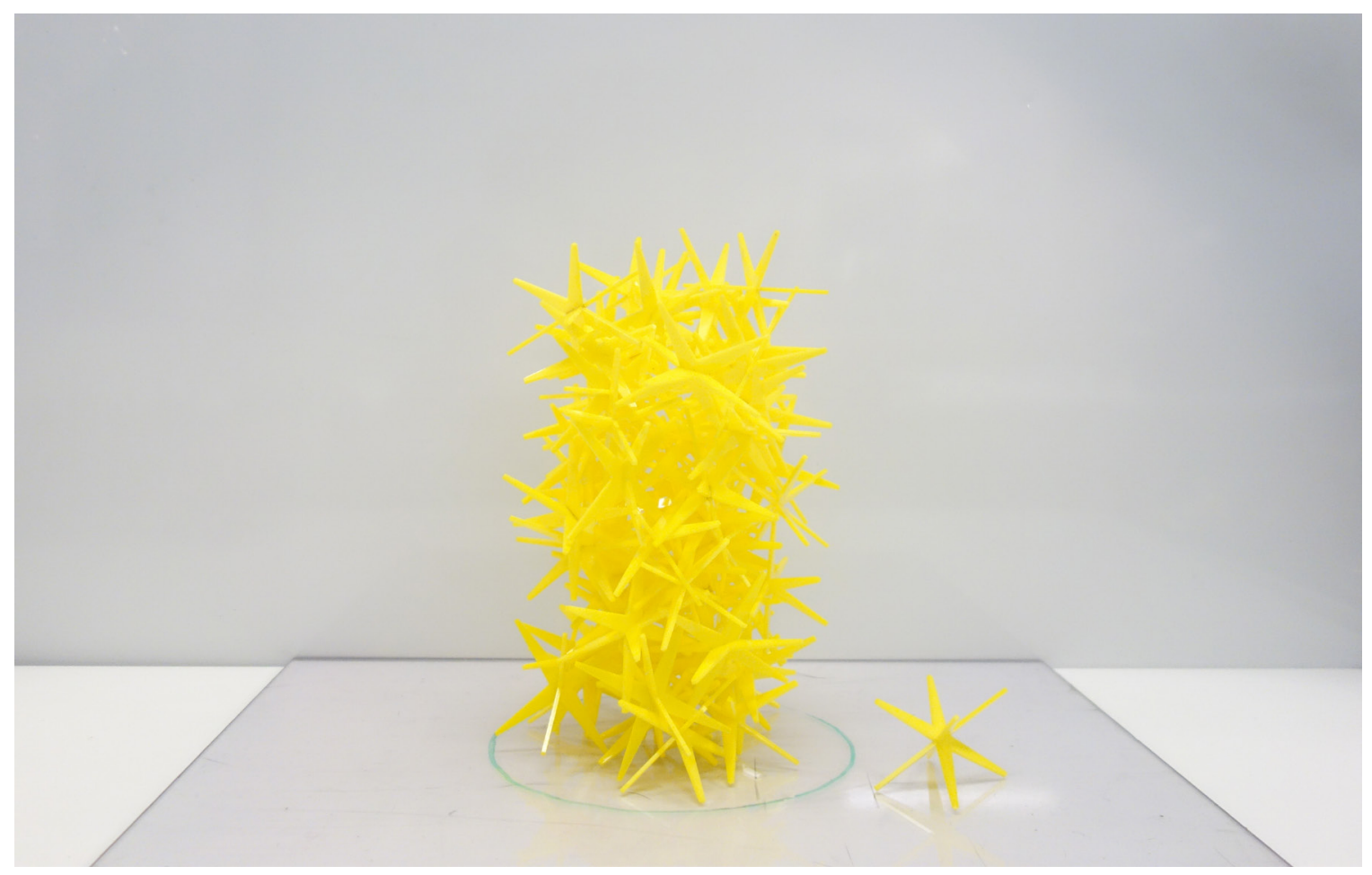

Figure 36. 74 pc, 8" d columns; acrylic particles

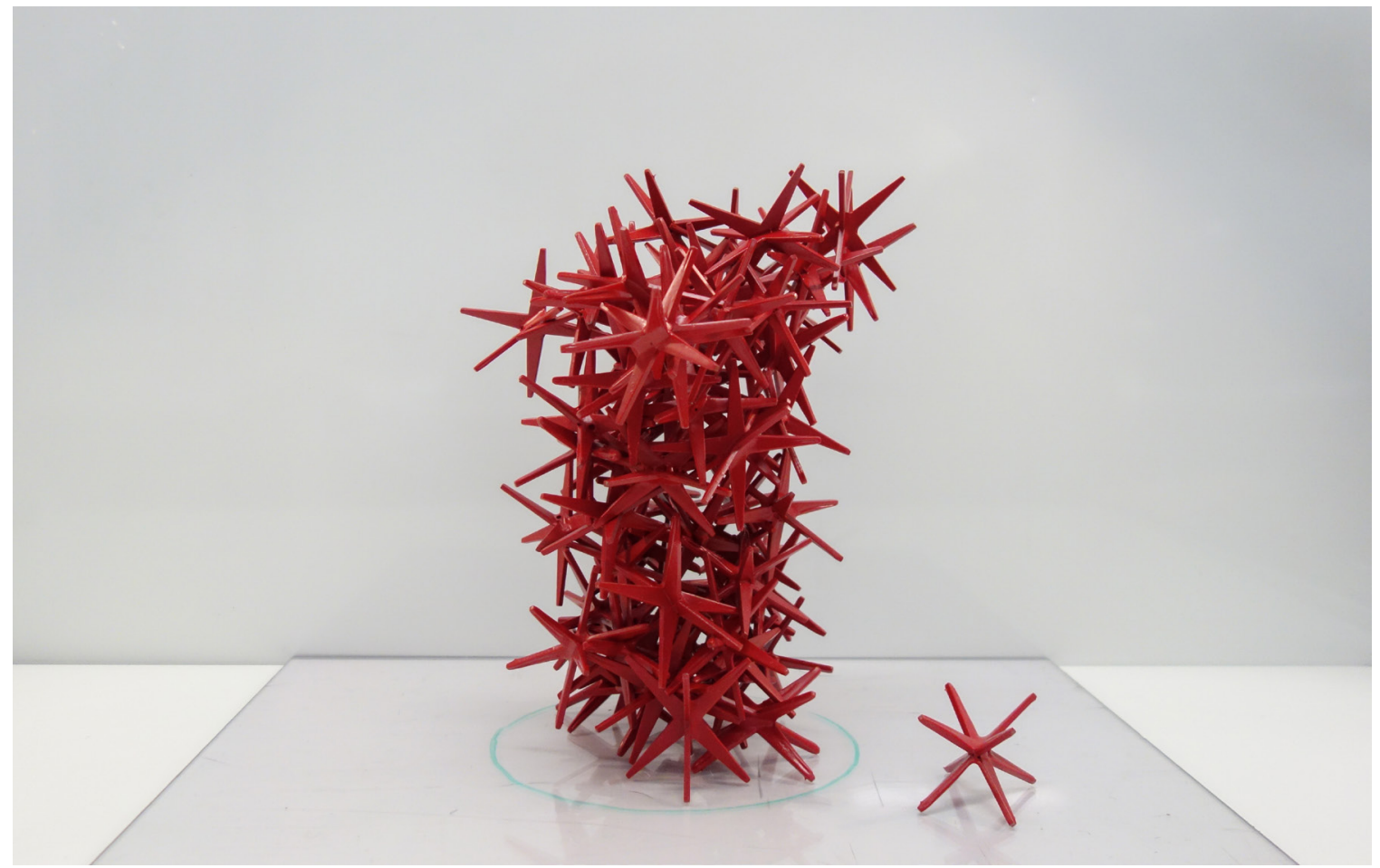

Figure 37. 74 pc; rubber coated particles 


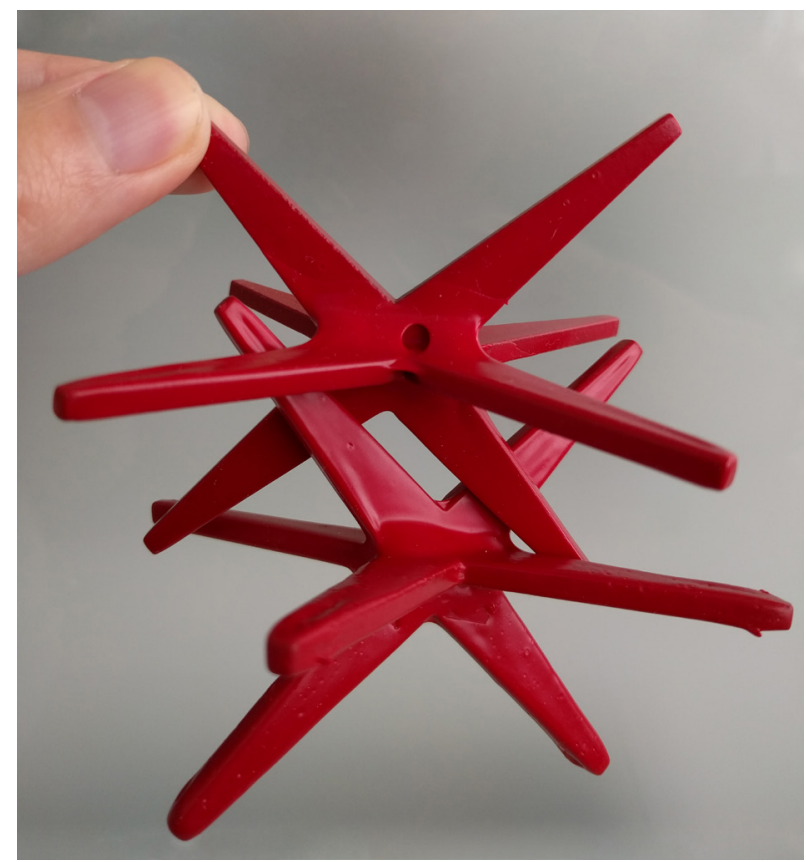

Figure 38. Rubber coated particle held together by material friction

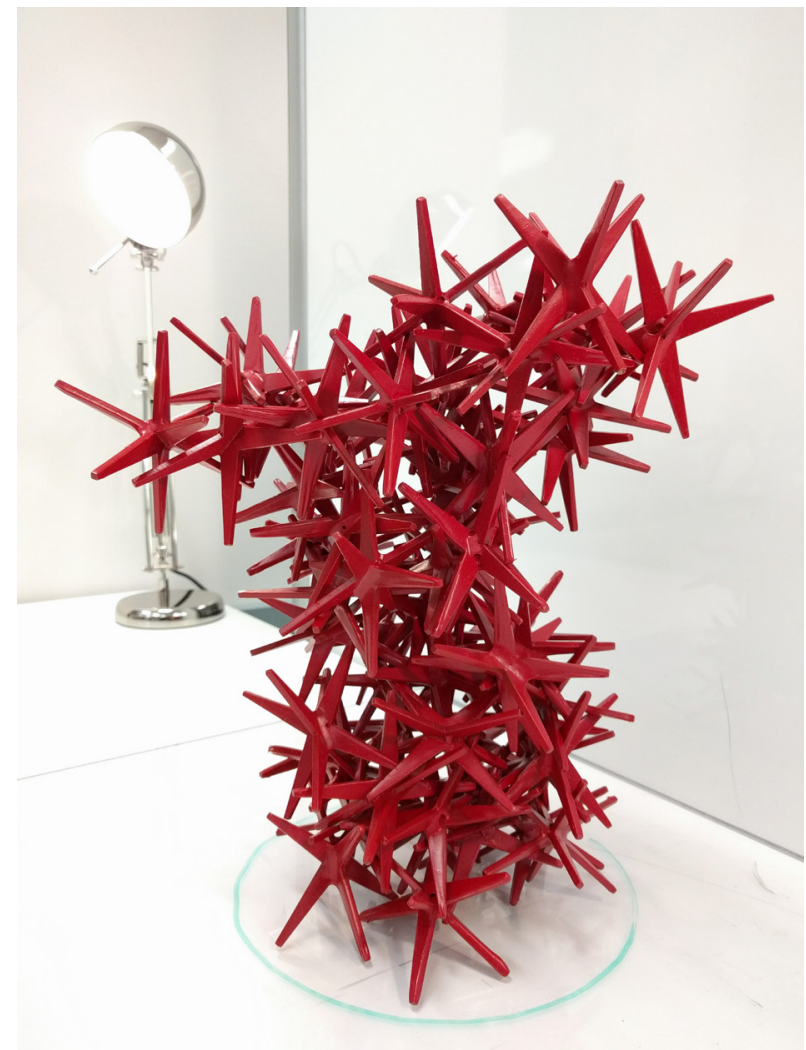

Figure 39. Form with greater projecting elements enabled by greater material friction

66 Architecture at the Edge of Chaos 


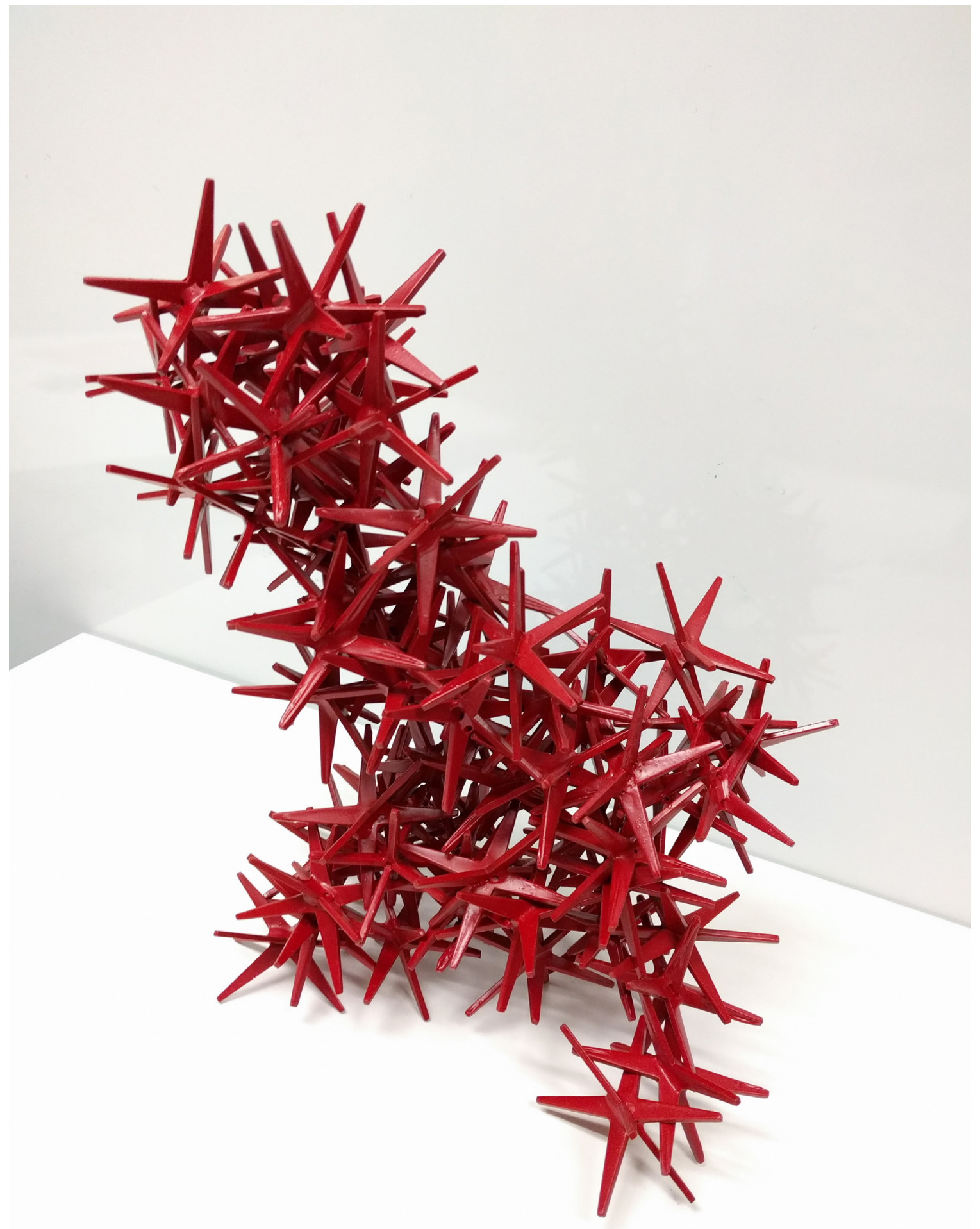

Figure 40. Greater formal possibilities with rubber particles 
Geometric interlocking, together with material friction can contribute to high stability, but material friction on its own-relative to geometric interlocking is not as secure. It is very easy to dislodge a particle held only by material friction. Further, while movement between particles is greatly restricted limiting slipping with high friction material, this also has the ability to resist vibration, which has been shown to increase stability.

Table 6. Effect of material (friction) on pile stability

\begin{tabular}{|c|c|c|}
\hline Material & Friction & Pile Stability \\
\hline Acrylic & Low & Low \\
\hline MDF & Med & Med \\
\hline Rubber & High & High \\
\hline
\end{tabular}

Again, a combination of particles of varying friction can be used. Figure 41 illustrates a column with a stable base consisting of rubber particles with a more fluid upper portion seen with a large proportion of acrylic particles. 


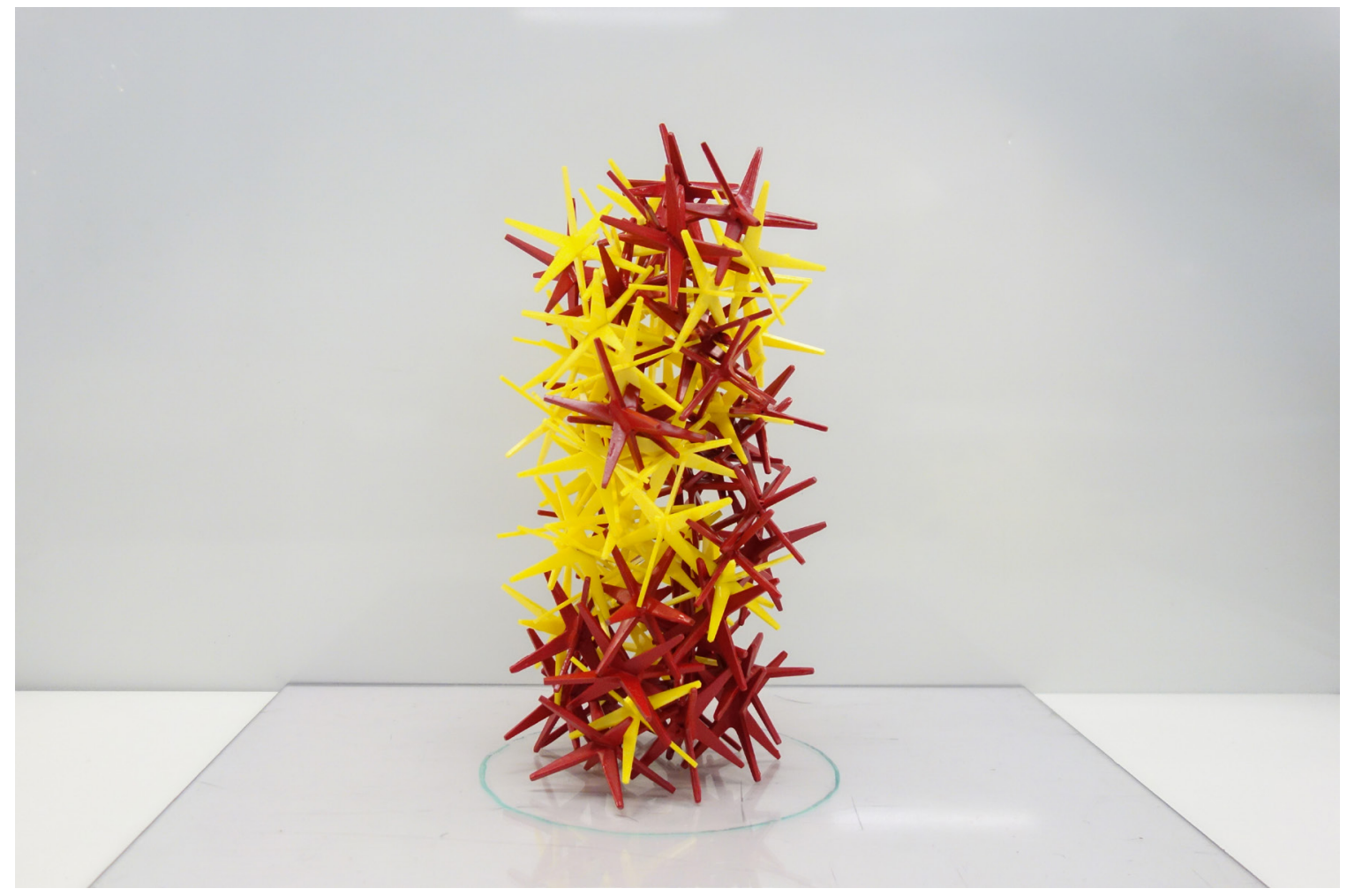

Figure 41. Mixture of acrylic and rubber particles 


\section{Surface / Arm Articulation}

A limited study of arm articulation was conducted. Variations included adding "bulbs", "teeth", or "hooks" to the arms, which contributed to different interlocking ability than the base module (Figure 42). The idea behind the bulb particle was to create an obstruction at the tip of the arm in hopes that the bulb would create a "knot" within the web of arms preventing the particle from being dislodged. The obstruction must be carefully designed as it may have the effect of inhibiting interlocking. The serrated particle introduced teeth along the length of the tapered arms. The teeth were shaped to encourage easy penetration into a pile, but make it more difficult to slip around or out once engaged. The teeth would catch on and hook on to the rough edges.

This led to the development of the hook particle (Figure 43). A hook was placed at the ends of the particle arms. It acted similar to the bulb as a form of obstruction, but also performed like in the serrated type to hook on to the arms. Excitingly, the morphology begins to introduce great tensional capabilities as the particles are now able to pull on each other (in addition to compression). Figure 44 demonstrates an experiment where a rod was inserted beneath the centre of a column laid on its side and slowly lifted. The acrylic particle pile was the quickest to break, while the rubber particles, due to its high friction stuck together longer. The hook particle while initially would start separating, the hooks eventually engaged in tension increasing cohesion significantly.

As both tensional and compression forces are now considered it offers greater possibilities and relevance for full-scale architectural structures and applications. In terms of formal possibilities, much more significant cantilevers can begin to be achieved that no longer rely purely on compression strength (Figure 45 and Figure 46). However with this there are other methodological implications. The aggregated mass cannot so easily be dissolved, which is good for reliability and stability, but greater effort is required to disentangle the components. Hooks (double convex shapes) and their methods have started to be investigated by others (Dierichs \& Menges, 2016). 

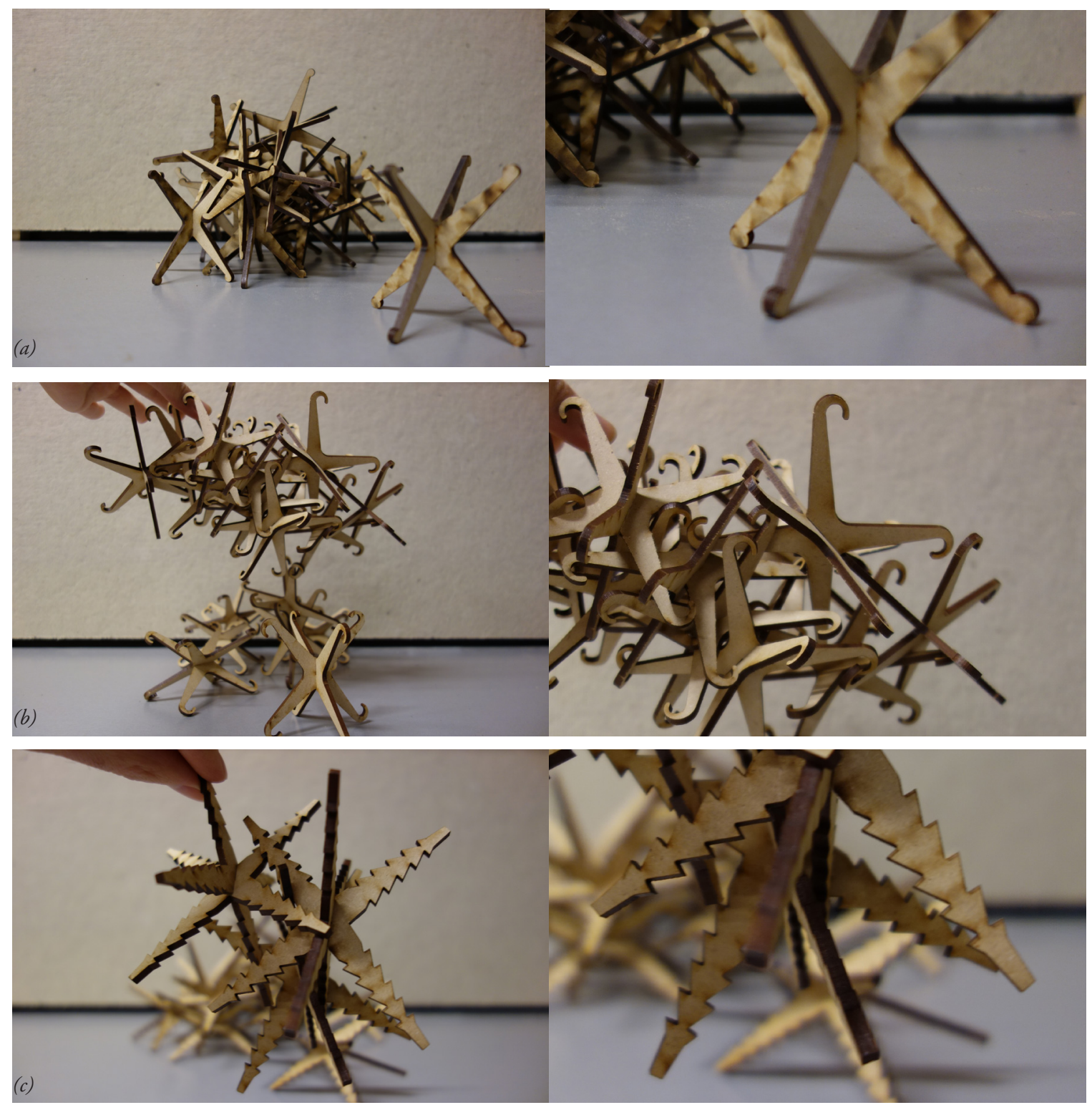

Figure 42. Different arm articulations: (a) "bulb", (b) "serrated", (c) "book" 


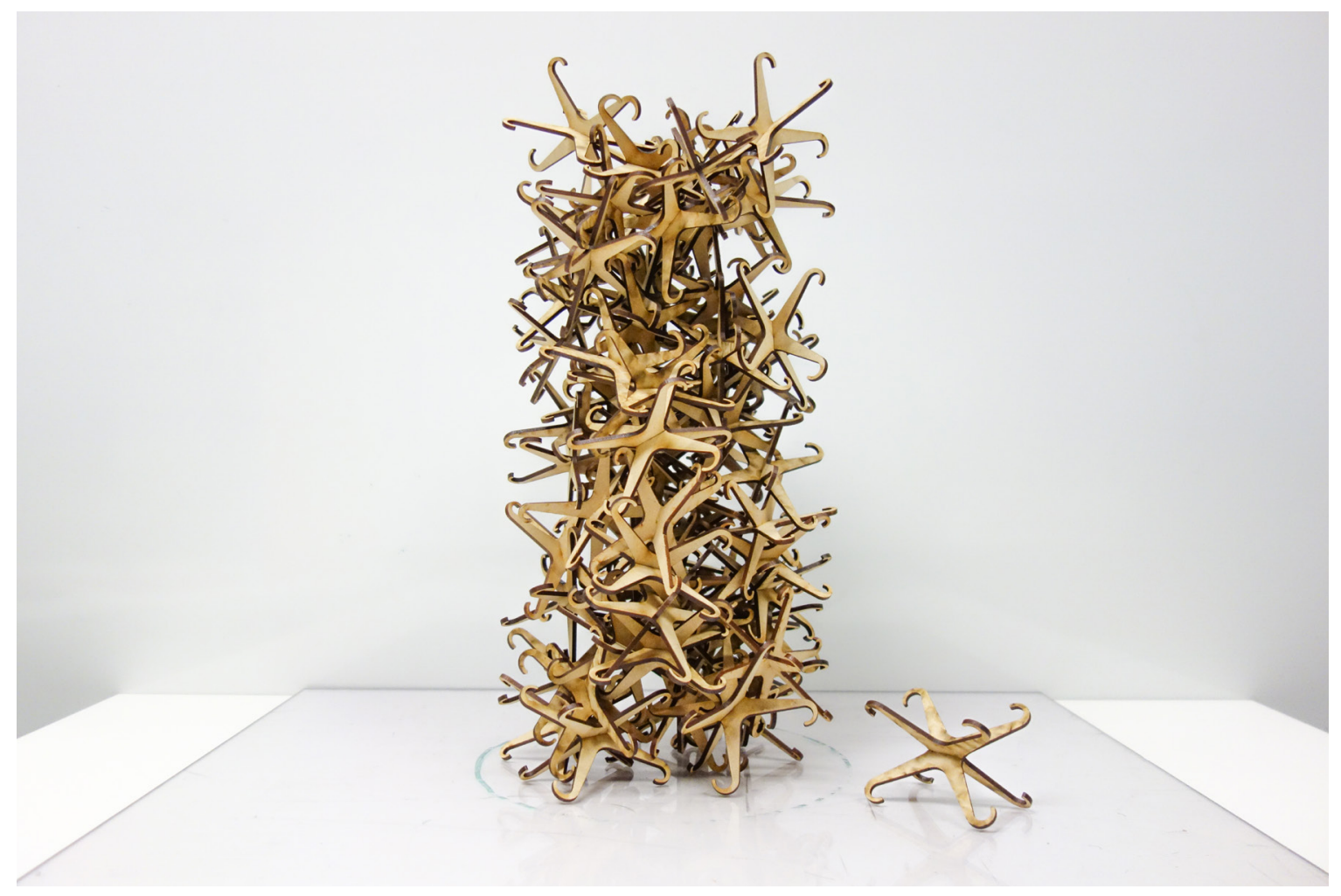

Figure 43. 74 pc hook particle 

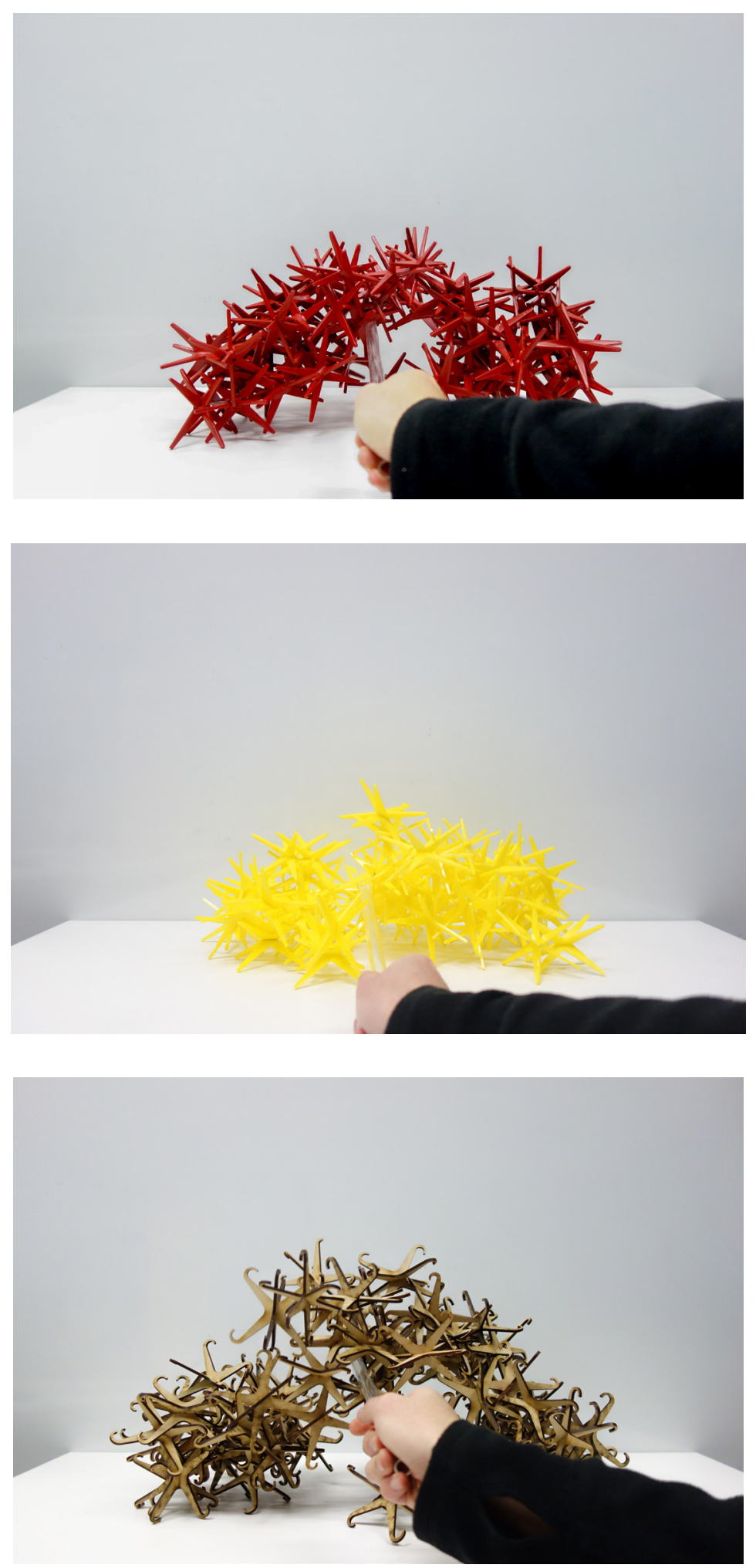

Figure 44. Testing cohesion of various particles 


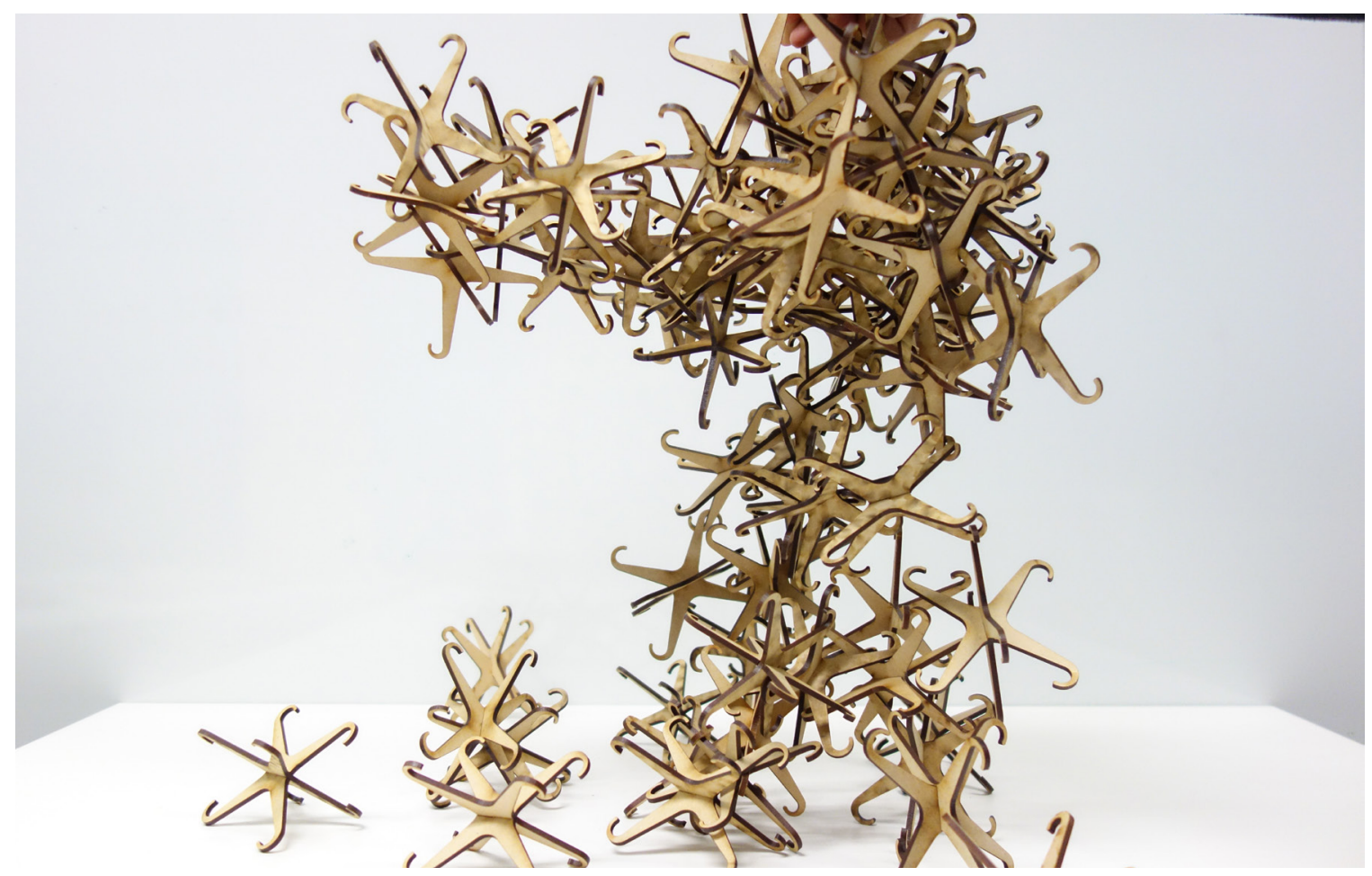

Figure 45. Hanging formation made possible by hooks

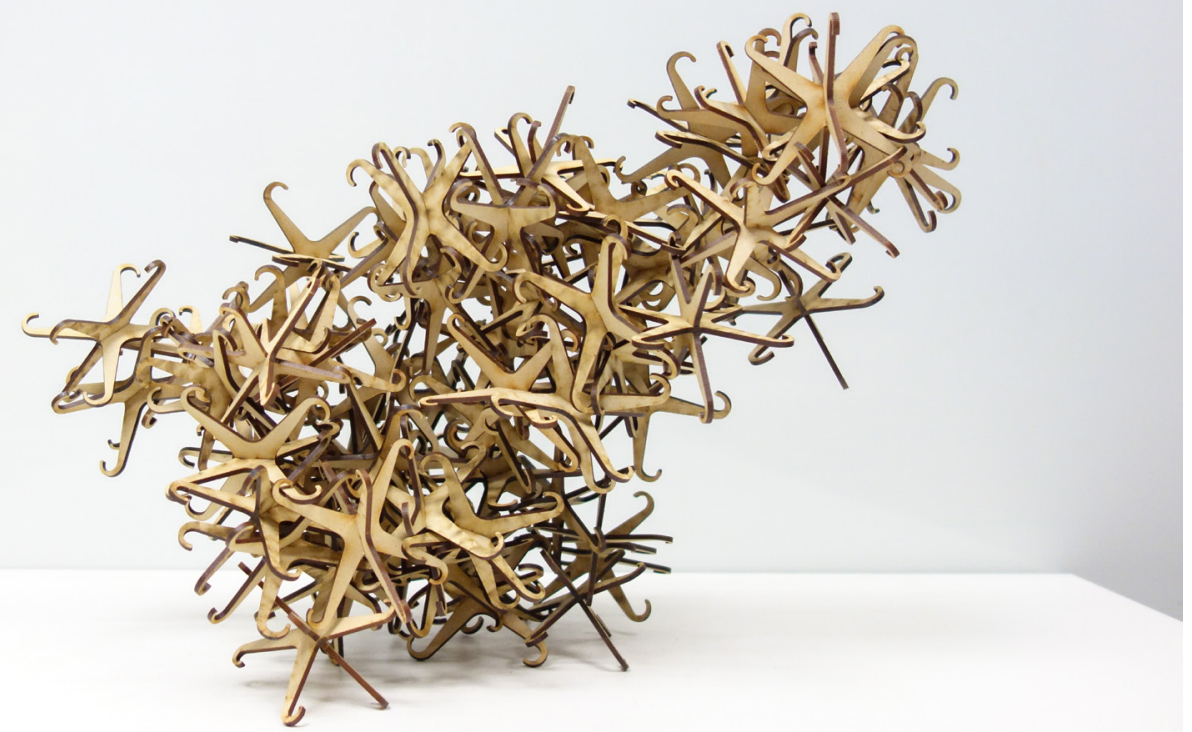

Figure 46. Hook particles able to withstand tensional forces is capable of forming large cantilevers

74 Architecture at the Edge of Chaos 


\section{Particle Design Summary}

Through variation in geometry and material, a range of behaviours can be calibrated for specific architectural qualities and forms upon aggregation. The studies thus far conducted in this field have been mainly on aggregate systems consisting of a single granule type as these are complex systems that are only starting to be understood. Mixing a variety of different particle types have been attempted in this work. Grading the system by mixing particle morphologies provide another level of tuning and possibilities, and with that even greater intelligence to address different complexities. The tests conducted here demonstrate the critical relationship between local element and global formation. Small, but precise changes of components were found to can have large impacts on overall form and behaviour.

Figure 47 and Figure 48 show an attempt at a cross-category structure using some of the notable performers discovered in the investigations. A cantilevering arch shape was attempted where the hook particles were employed to create a tensional top layer, while the highly stable rubber particles were to take on compression on the underside. Both stability and malleability can be achieved with specifically designed particles to produce forms not capable with natural granular materials yet still assuming its fundamental characteristic - its loose property and thus its ability to be built and rebuilt.

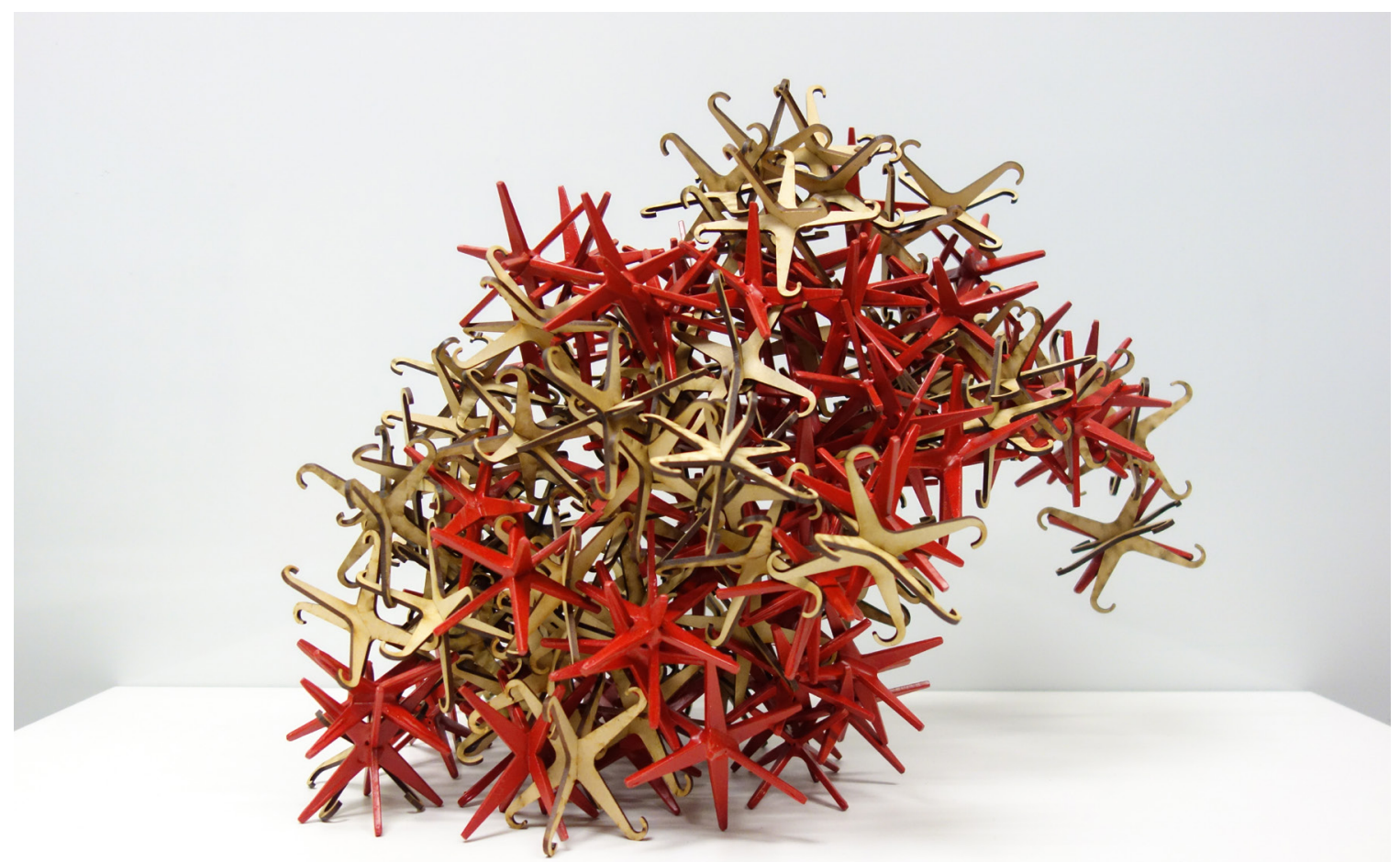

Figure 47. Mixture of rubber and hook particles attempting a cantilevered arch 


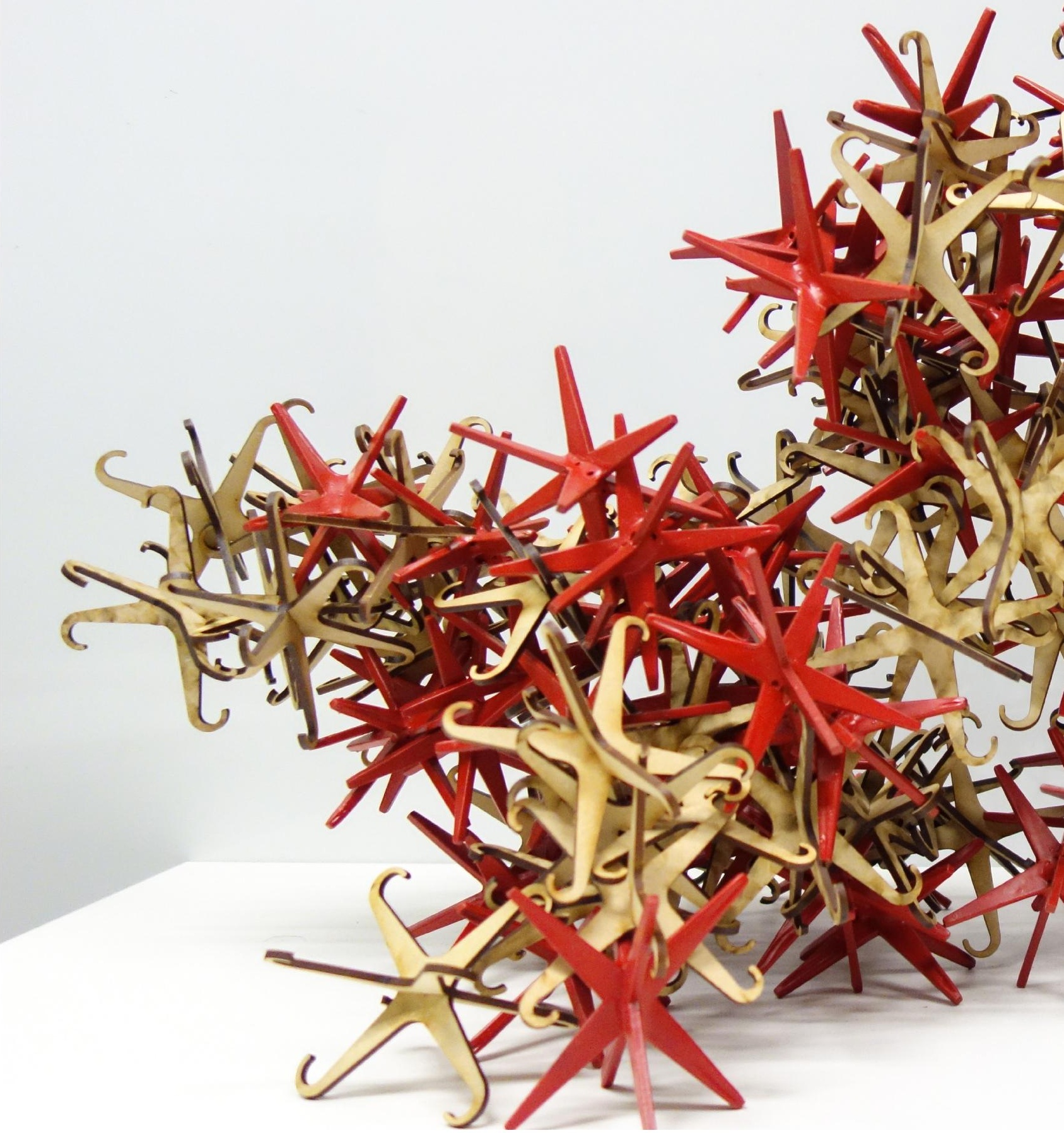

Figure 48. Expressive formal possibilities can be afforded with the right mix

76 Architecture at the Edge of Chaos 


\section{Aggregation Processes, Methods and Application}

For the following empirical explorations, approximately 1,100 of the base particles were created. By committing to a particle morphology, the focus was to explore issues of architectural application, processes, methods and tectonics. Based on the system's demonstrated ability to quickly assemble into various forms and proto-architectural elements such as columns and walls that are able to withstand load, this led to an interest to further explore more sophisticated forms of enclosure. While beginning to consider the material in an architectural context, it was important in these investigations to keep in mind the scaling of methods and technologies used with an overall goal of simplicity and accessibility.

\section{Deposition}

Numerous experiments were conducted to test the ability of the system to aggregate, enclose and vault (Figure 49). Deposition of material was carried out by hand, varying the degrees of user influence. Unregulated deposition was carried out by random, unbiased addition or mass "dumping" of material. This was not found to be very successful in creating desired forms or vaulted structures. However, to achieve desired forms, influence on deposition was found to be required through a continuous feedback process of observation and adjustment/compensation (Figure 50). This real-time design process is a unique aspect to working with this material. 


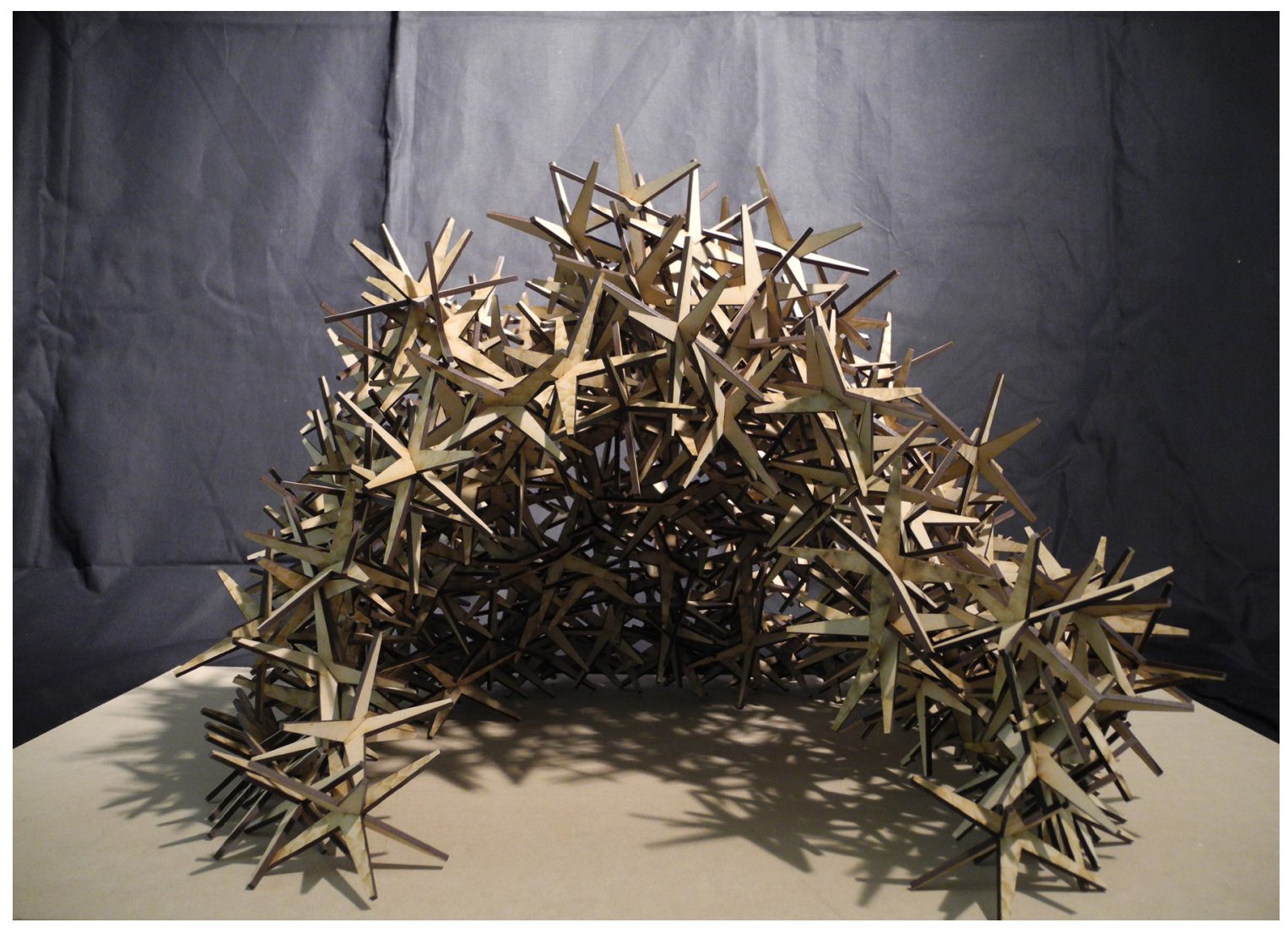

Figure 49. Vault enclosure 

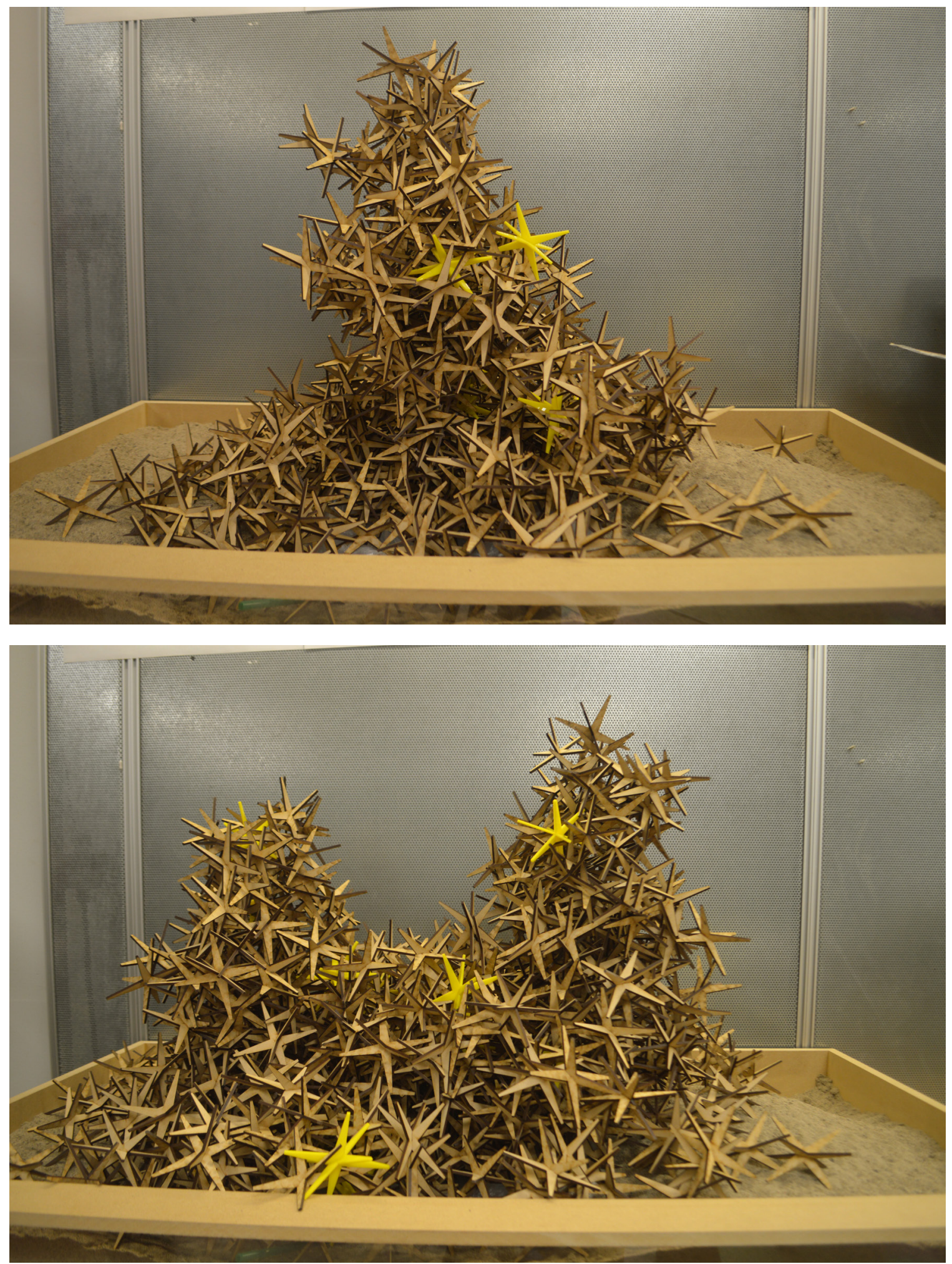

Figure 50. Continuous feedback and adjustment is required as the processes of design and building are blurred 
Influence on deposition is distinct from the organizational property of the system. It does not imply the total ordered placement of components (although that was also tested), but guidance is provided on the distribution of material yet the system is free to self-organize. In trying to achieve a greater degree of control, for specific features such as openings for example, a hybrid ordered and self-organized method was tested where stacked elements served as a framework to guide the formation in key areas of the structure (Figure 51).

Furthering the explorations spatially, different spaces and processes were developed at imposed working scales of 1:10 - 1:5. By changing the working scale of the structure, the actual size of the particle can be kept the same but conceptually be changed relative to the structure. By operating within a scale, actual factors of process and construction were brought to the fore. It was with this that the quantity of components that would be required was determined, which necessitated considerations of how to actually handle such material. Pneumatic systems were introduced into the experiments as a way of guiding (controlling) form and as part of an integrated hybrid assembly.

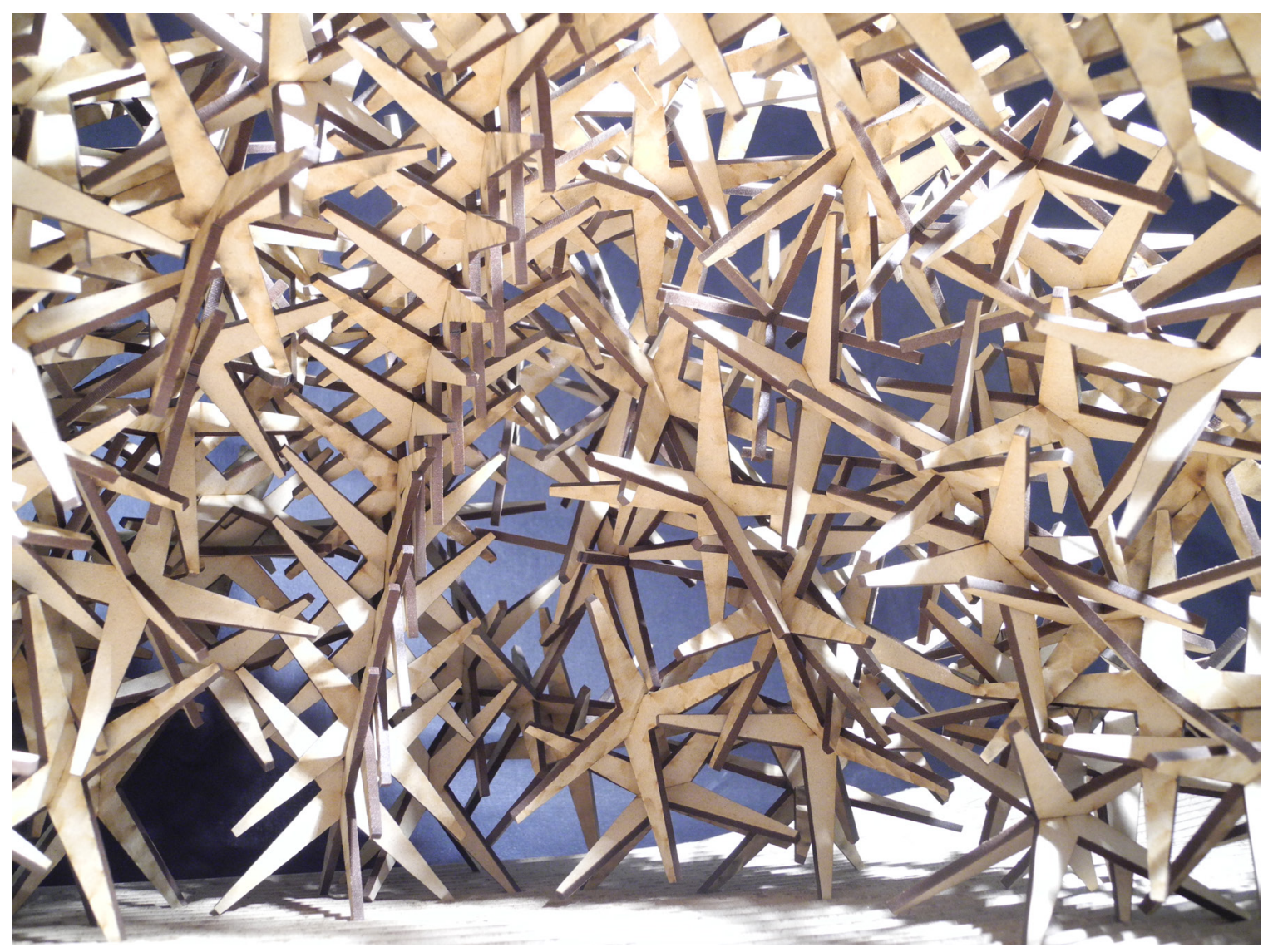

Figure 51. Close up of hybrid ordered-self-organized enclosure 


\section{Formwork}

The aggregate system was explored in conjunction with pneumatics with the intention of perhaps developing a hybridized material system and construction process. The creation of different inflatable forms acting as falsework allows desired volumes and spaces to be created through the displacement of the aggregate material (Figure 52). Through the adjustment of the inflatable forms the volumes can be continuously manipulated and updated.

The falsework, once deflated, can be easily removed and reused. On the other hand, the option of keeping the air structure inflated and making the inflatable portion inhabitable offers the aggregate system a way to overcome potential issues associated with porosity: air and water tightness. Although, a denser or thicker aggregate composition may also be a solution for those issues. Working with air was a natural fit; as a material, it is even more malleable than the aggregate system providing unrestricted, maximum control over the loose system, while the pneumatic membrane as a supplementary component compliments its performance. Where inflatable structures are light and portable but lack solidity and mass, the aggregate system has the ability to compensate for it.

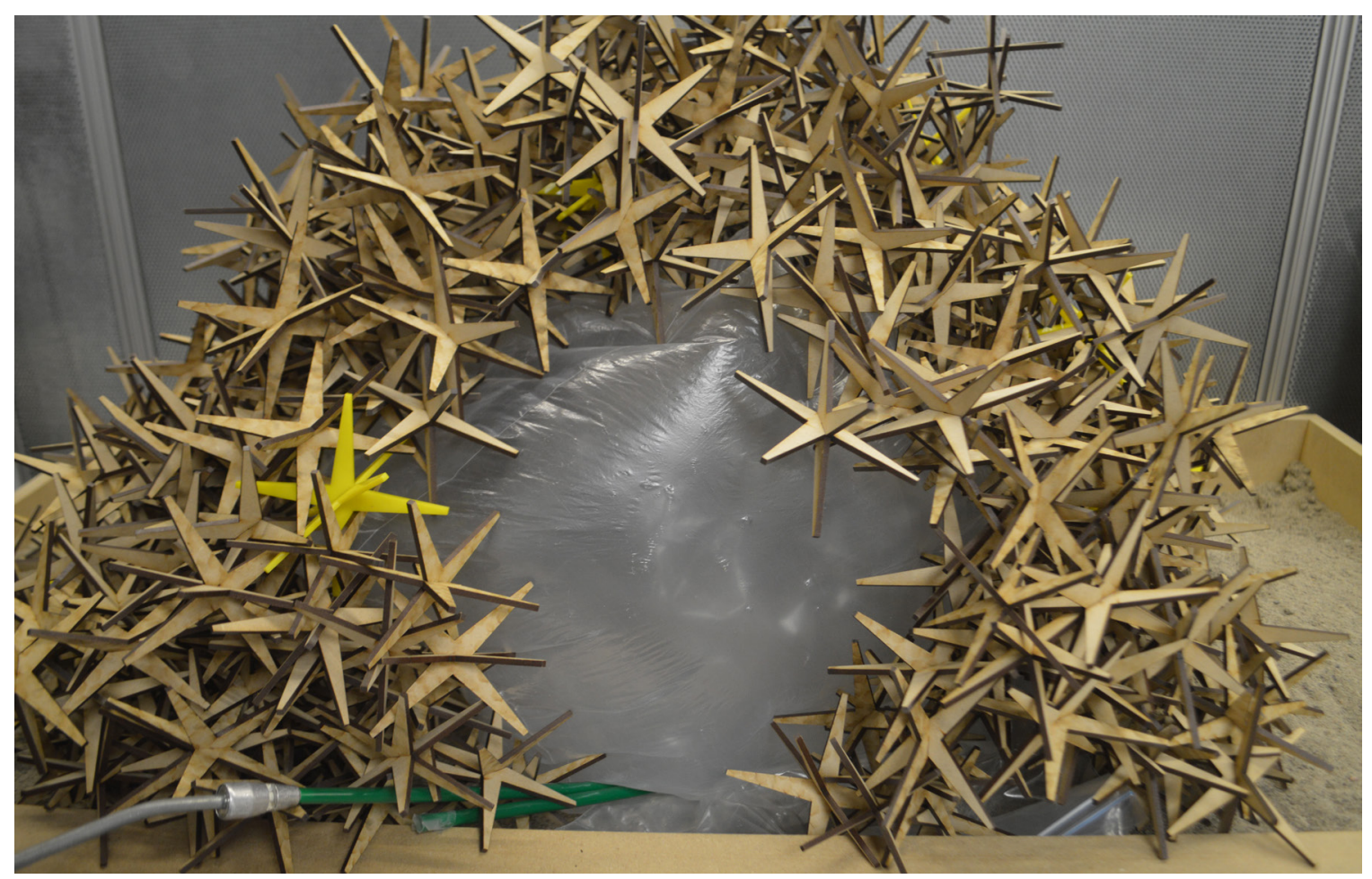

Figure 52. Inflatable formwork 


\section{Binding}

The distinct advantages of the aggregate system are a) it is loosely assembled and b) it requires no special connections. Although, it was made clear through discussions with a structural consultant that the system as a concept and especially as an architectural material is far from being understood and thus its stability is reluctant to be accepted. The response was understandable, new procedures and rigorous empirical tests would be required to validate and create guidelines for modern day use to ensure public safety.

As such these discussions initiated a series of tests exploring methods of binding the components together for an additional level of stability. Paramount to the concept of binding within the context of this system was to maintain its fluidity and thus the mechanism had to be reversible and still accessible (simple). String was investigated as a binding agent in two ways. First, an array of particles was created by loosely linking particles together using string (Figure 53). This made handling easier and quicker to deploy as it was grouped into one object. Connected by string, the particles could only behave semi-autonomously when compared to the unbound system, which could be seen to create some instabilities structurally. The result introduces a new tension force that would sometimes create "floating" particles that were solely held up by the string and did not naturally fall into place (Figure 54). If regarded as a fully integrated part of the system itself, adding the tensional capabilities to a system that excels in compression would mean some trade-offs but would extend the usefulness of the system.

In the second scenario, string was used post aggregation to loosely envelope and bundle the system serving as a redundant layer (Figure 55). The advantage here as a distinct add-on system, is the string could be removed and the system would be free to reorganize. However, while binding attempts to confine the particles from moving leading to potential failure, it can also restrict the system's ability to adjust and equalize. So while it can be kept quite solid and stable it may not be in the most optimal state relying perhaps excessively on the secondary string layer. 


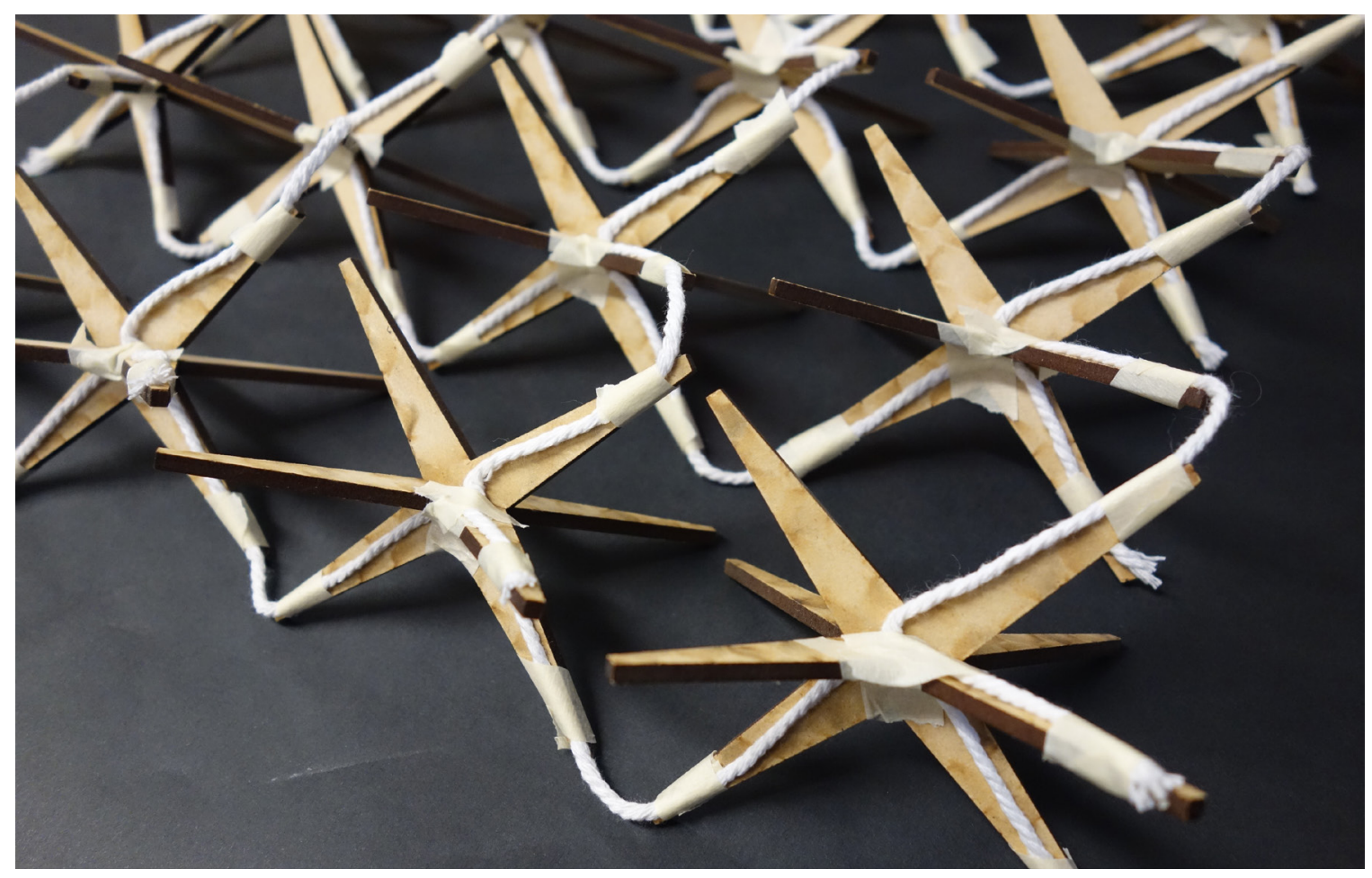

Figure 53 Array of particles linked with string

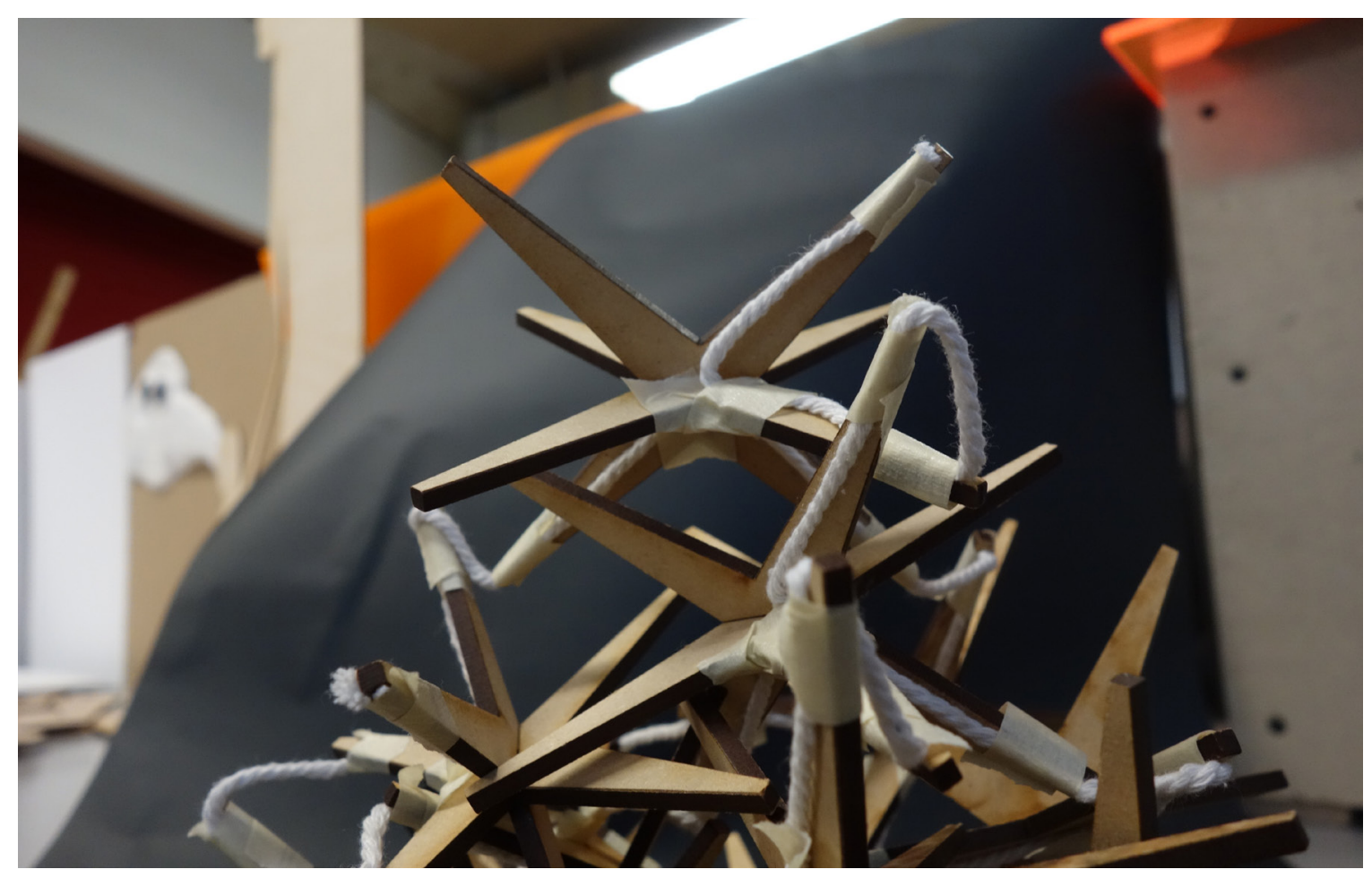

Figure 54: Top particle is held up fully by the tension of the string 


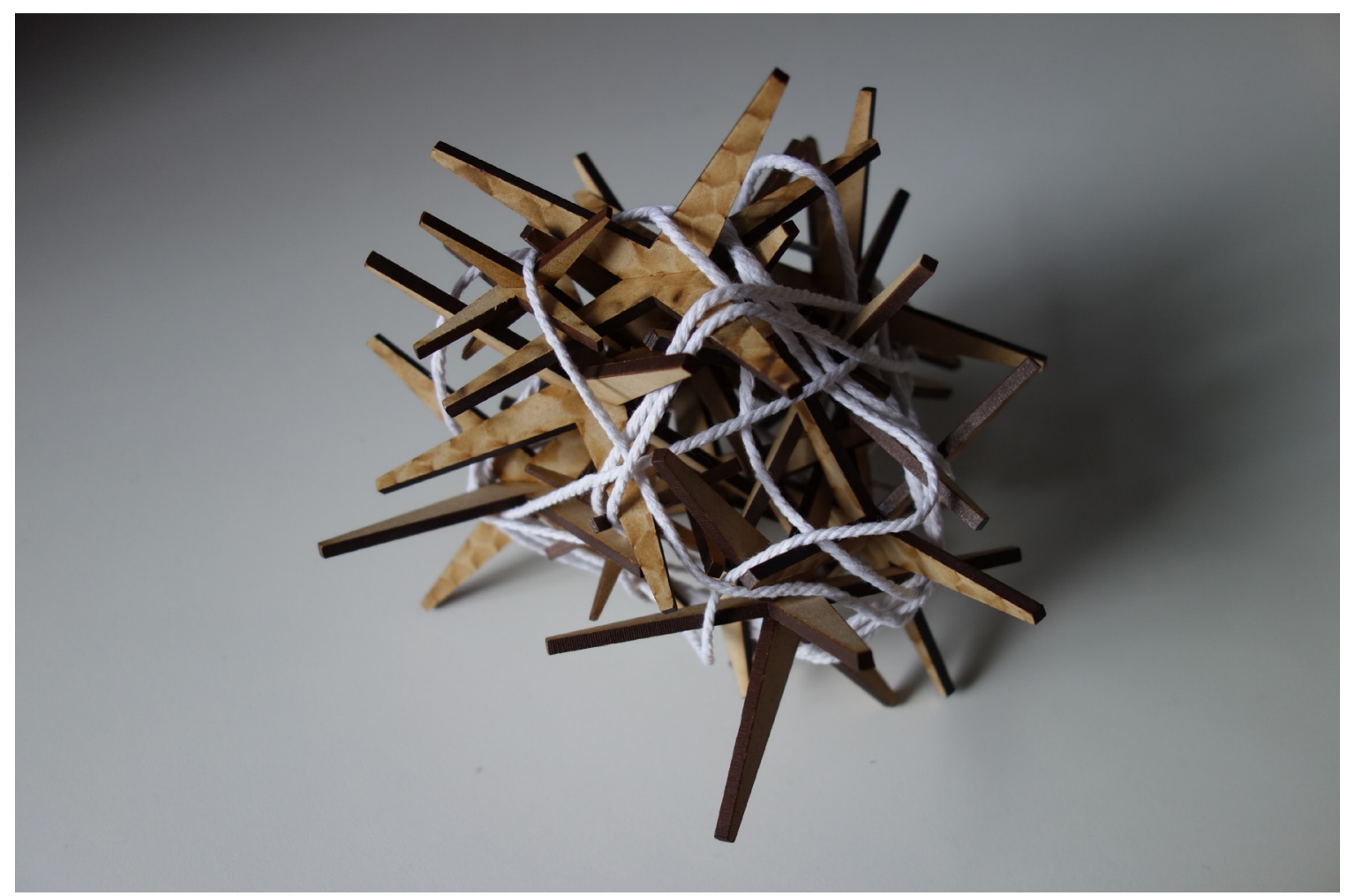

Figure 55. String binding post aggregation 


\section{Vacuum Jamming}

Vacuum jamming is technology that induces jamming of a granular material via removal of air from a membrane container containing a granular material. Once jammed, the object becomes solid-like, but can become liquid-like once again through the introduction of air. This is employed in the packaging industry for products such as coffee, although for different purposes. V-process is a variation to sand casting utilizing vacuum molding to hold granular material (sand) in a particular shape to produce complex free forms and is used in various industries. Recent research also has been conducted on vacuumatically-stabilized granular material as novel approach to creating an adaptive mould/ formwork for realizing topology-optimized structures/ components in architecture (Huijben, 2016). In these scenarios the granular material serves as temporary formwork for a separate system.

Continuing this transfer of technology, this investigation looked at utilizing vacuum jamming as a complementary system and methodological approach. Its ability to hold forms is promising structurally as it could provide a secondary form of redundancy to ensure stability. Most importantly, it maintains the systems reversibility. On performance, this hybrid system potentially offers good insulating qualities due to vacuum/ trapped air the particles create between the membranes. Furthermore the membrane overcomes practical issues of air and water tightness. Logistically, this container can facilitate ease of handling and work as part of the packaging and delivery method.

Air could be viewed as an archetypical soft material. As discussed, Negroponte and others such as Archigram, has examined or experimented with inflatables. In the context of architectural applicability its weakness lies in its flimsy structure. The proposed hybridized system seeks to compliments both technologies. The membrane or bag for the first time allows the units to be handled and regarded as a whole. The container becomes a framework which begins to reconcile physically and conceptually, the individual and the collective which may prove to be a necessary consideration. This particular framework privileges the unit and the individual/local relationships, but allows the whole system/community to absorb and adapt to disturbance.

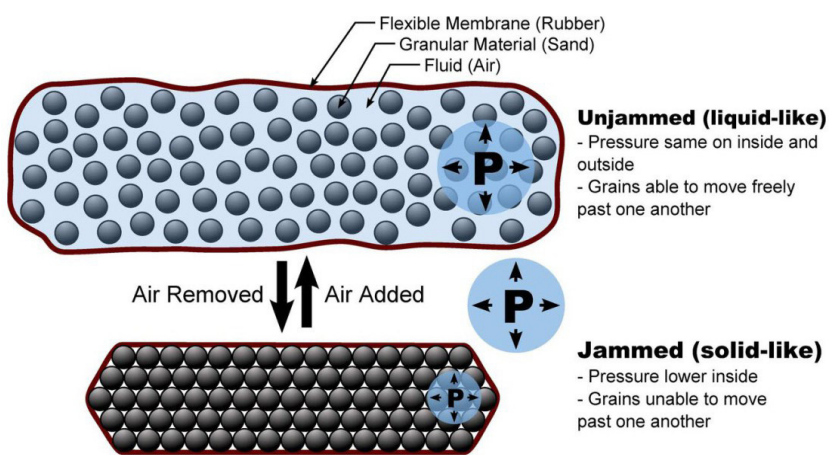

Figure 56. Diagram of granular vacuum jamming 


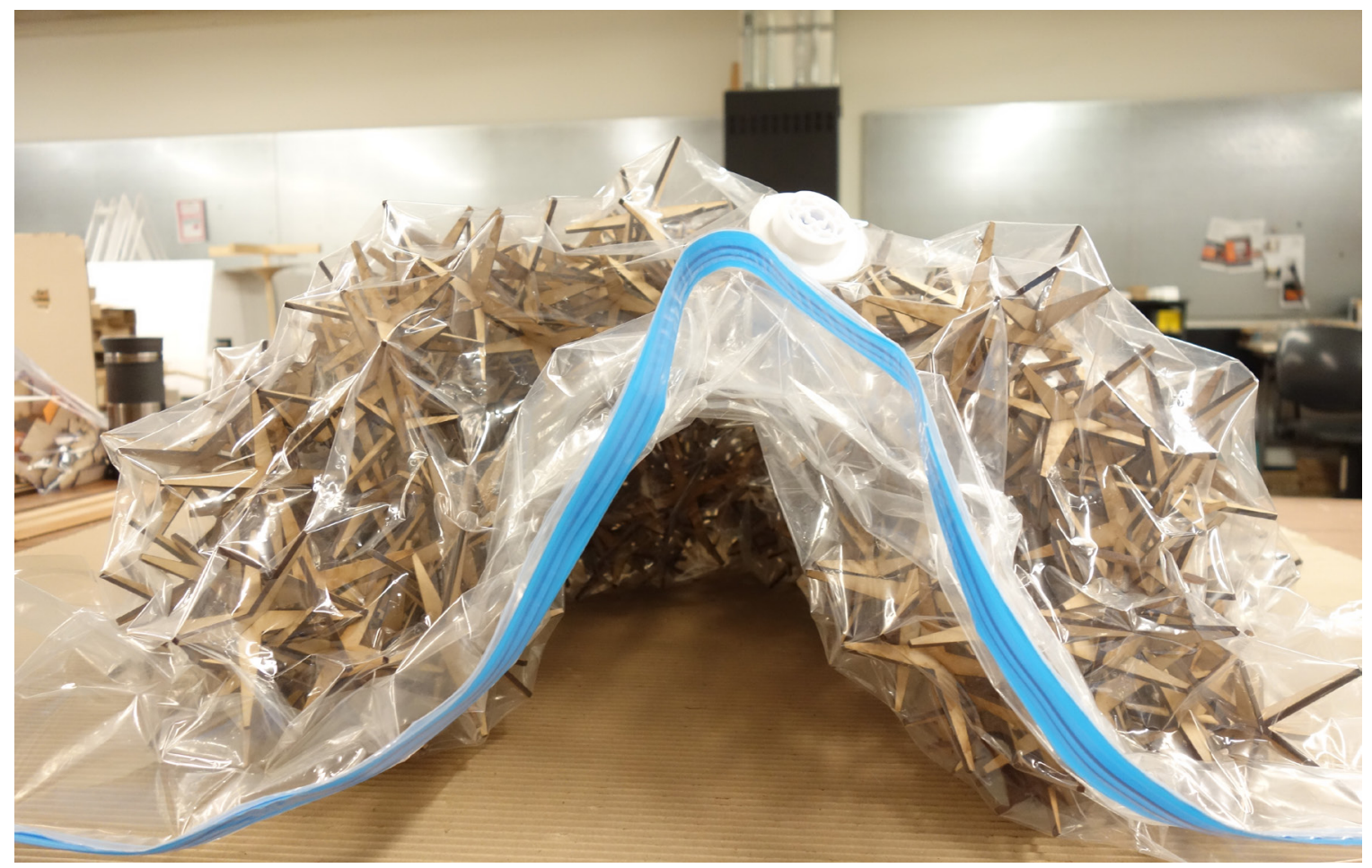

Figure 57. Hybridized system utilizing strengths of both systems

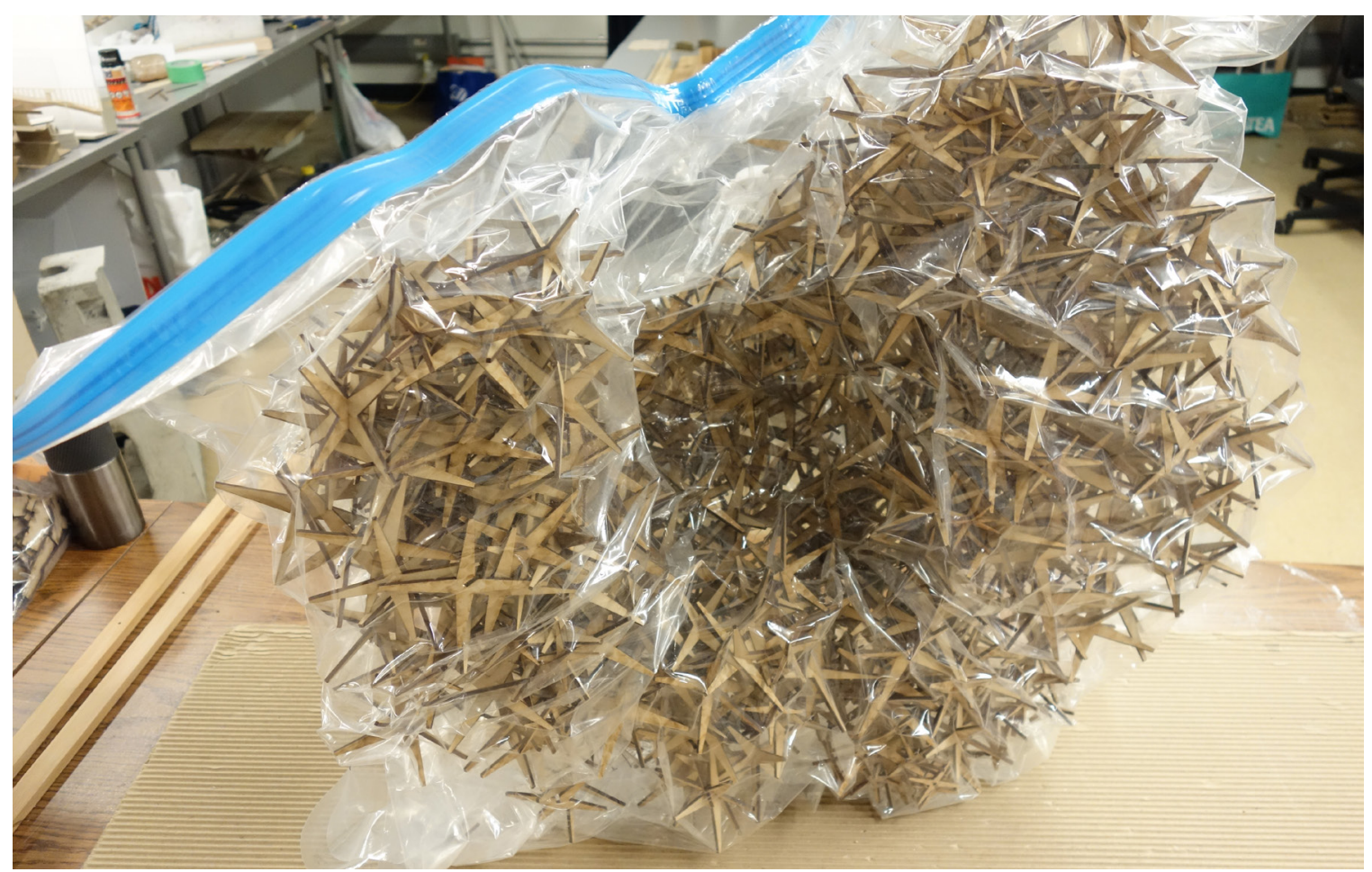

Figure 58. Particles jammed into a solid state 


\section{Scaling up}

The system was eventually scaled up by a factor of 5 . While the smaller components could conceptually be employed at a building scale, the shift to larger particles allowed for testing at a building scale with fewer components and an ease of handling. 1/4 inch thick birch multi-plywood was used and fabricated with a CNC milling machine. Due to the larger 2D geometries, the creation of additional smaller particles were possible using the leftover material and thus minimizing waste. This optimization led to a material composition consisting of graduated particle sizes: $25 \mathrm{~mm}, 50 \mathrm{~mm}$, and $75 \mathrm{~mm}$.

The shift in scale introduced new variables and complexities although the simplicity of construction was still an asset due to a lack of connections. Weight (dead load) was a factor that immediately surfaces as forces are multiplied. The system requires a balance of weight — a light particle is desirable for handling, but an enclosure depends on weight to provide the compressional forces necessary for stability. In this case the dead load of the particles is sufficient. It was also seen that external loading can have a strengthening effect.

Furthermore, with this shift in scale, production time increased not only as a result of producing larger pieces through different fabrication technology, but due to the material chosen, an additional step to finish the individual pieces was required prior to assembly. Once assembled from its $2 \mathrm{D}$ components, the volume of the batch, by its very nature, increased immensely and was fully appreciated for the first time. The volume has implications for the transportation of the material, which effects the overall production process. Finally, the particles were subjected to greater wear as the scale of particle collisions increased.

The larger system presented an opportunity to explore in different environments. Within an outdoor, natural setting uneven landscapes were encountered and it was exposed to live forces from wind. As expected, the system stood up to rough siting conditions. It was also able to withstand discrete, concentrated forces (projectiles) without unraveling. When constructed indoors on artificial, level floors the system faced difficulties with the smooth surfaces. The system-ground relationship has emerged as a key area that requires focus. Possible solutions considered include the use of gravel or embedding particles into the ground as a form of foundation/footing. 


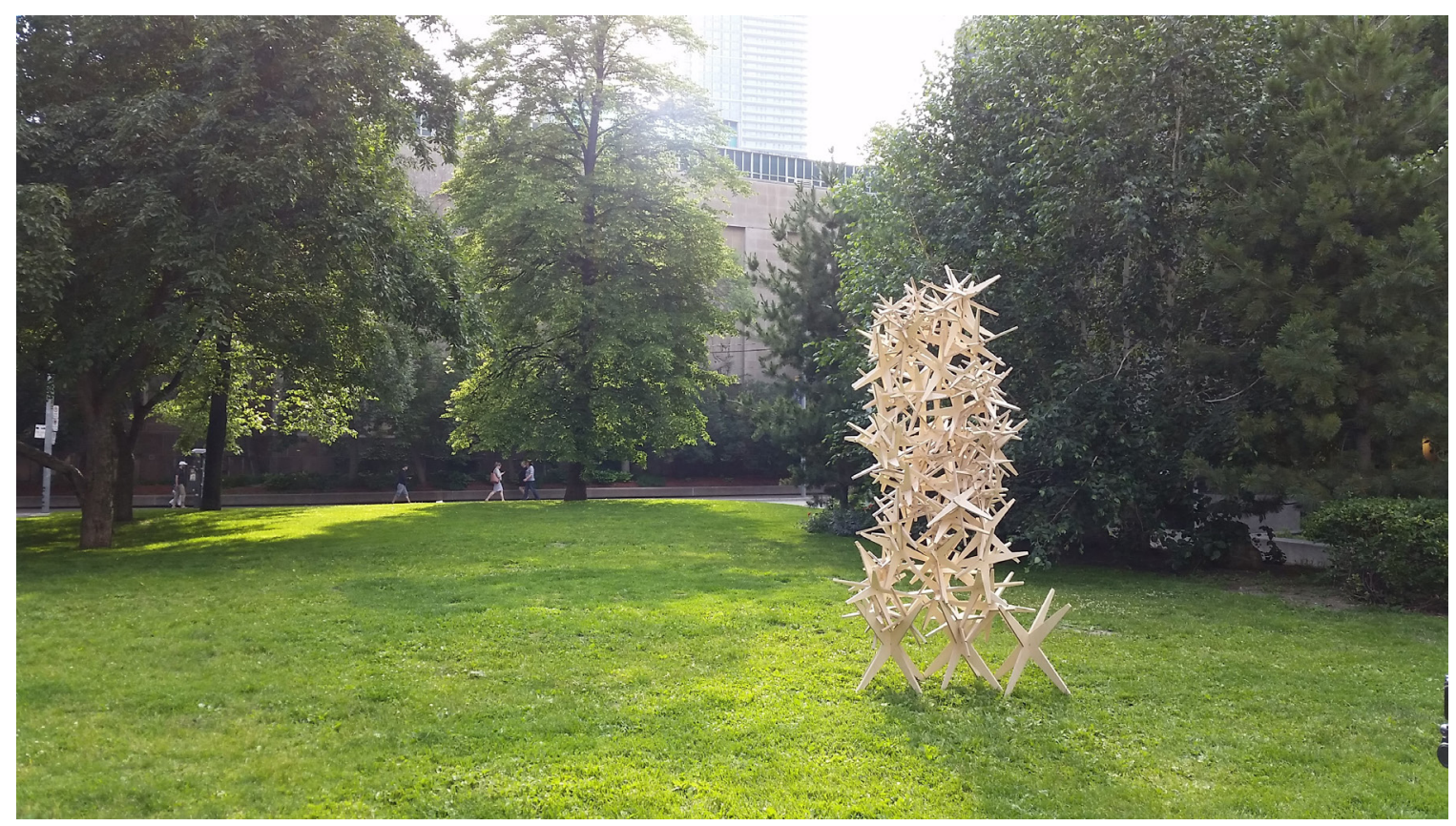

Figure 59. Large components assembled outdoors 
Different methods of aggregation was tested in a similar manner to the scale model investigations, but in this case without the use of pneumatics. A semi-controlled, monitored process rather than a completely haphazard or carefully positioned one strikes a balance between being quick to assemble yet still capable of producing, in general, a desired form. The material, consisting of graduated particle sizes produces a functionally graded material — that is, a material that varies in composition and structure over its volume that can result in corresponding changes in the material properties (Dierichs \& Menges, 2012b). Larger particles could be thought to provide the overall structural functions while smaller components served to fill in and provide local modulations. The material can be graded with different proportions allowing for a fine tuning of different surface properties such as malleability, smoothness, and porosity.

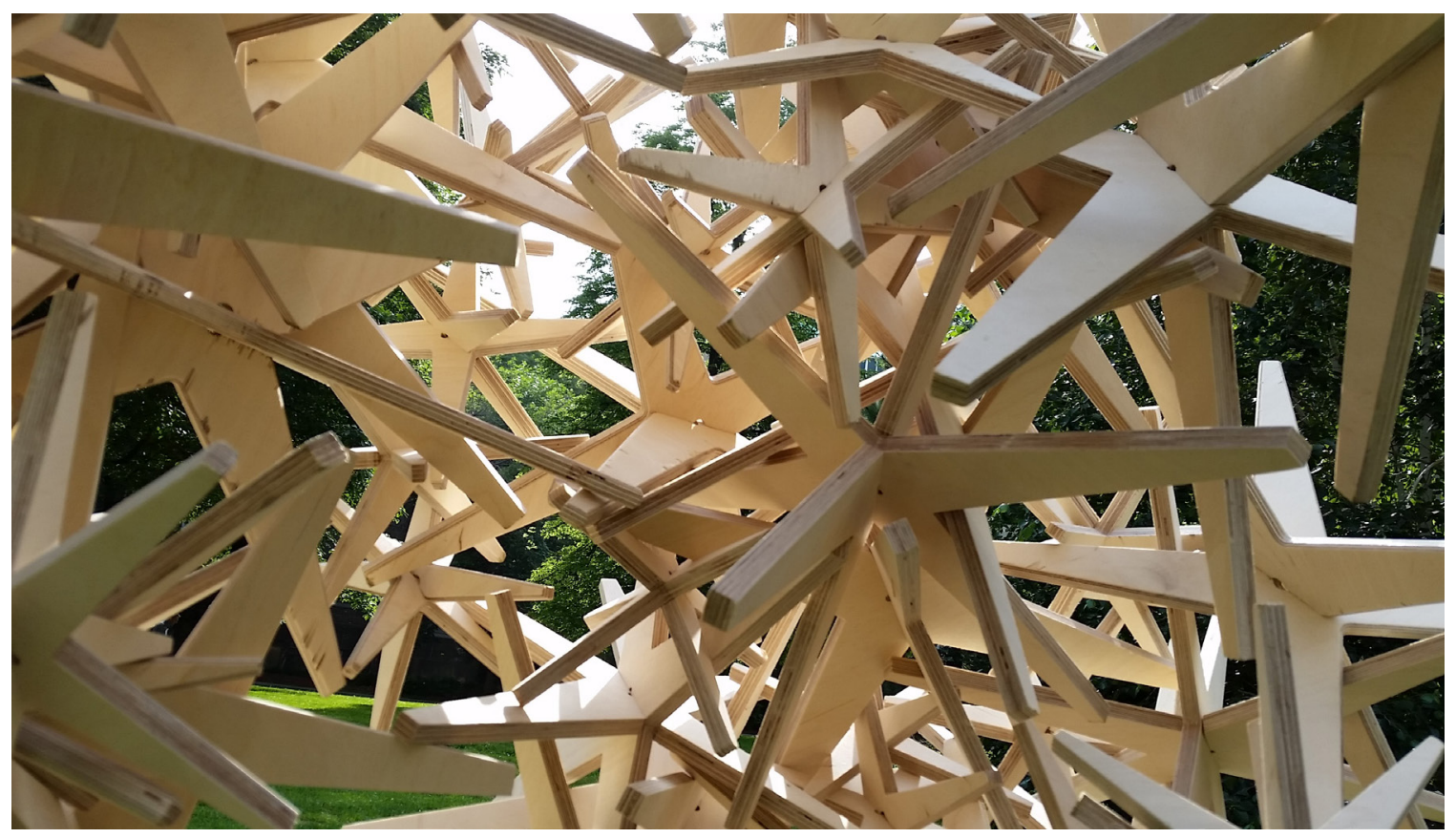

Figure 60. Detail of large components 


\section{Digital Tools and Representation}

In parallel with physical testing, different methods of digital testing, registration and representation were also investigated. The limits of laser scanning and the manual method of using a 3D digitizer to create a digital model were assessed. The complex geometry posed difficulties for the digitizer becoming labour intensive and susceptible to human error, while the non-convex forms were difficult to record with laser scanning. In terms of form generation and representation this presented a great challenge. The point cloud approximation method via Rhino with Grasshopper provided a fast, although non-physically accurate representation, to approximate the form. On the other hand, rigid body physics simulation using animation software (Blender) takes into account factors such as weight, friction, collision, and forces, but requires intensive computing resources and time for a more accurate representation (Figure 61). The accuracy of generating the model correctly-accounting for all the variables and moving parts of each particle_-is critical and any minor deviations can generate highly divergent results. This makes knowing the precise outcome/ form extremely difficult and they rely on statistical descriptions of its properties. This is akin to predicting the weather (which has become more accurate as data analysis technology has improved) but at this time it remains very difficult if not impossible due to the vast quantity of variables.

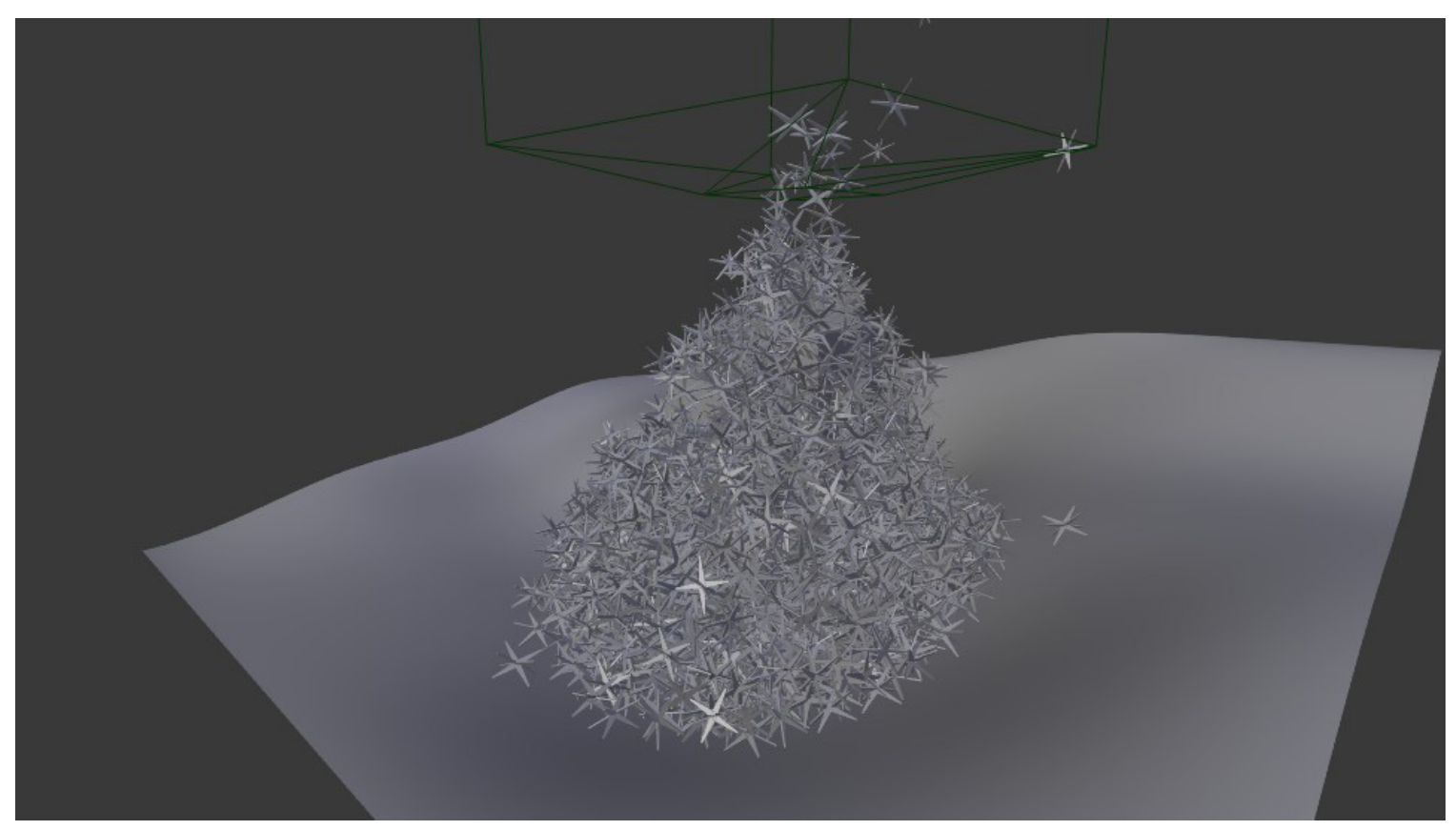

Figure 61. Physics rigid body simulation using Blender software 
The conventional desire for a digital model is for a tool to convey design intentions where it may be difficult or not feasible to show through physical models or drawings. In this context, working with granular materials, where gravity and forces are inextricably linked to the form and outcome, challenges how architects design structures currently in gravity-less virtual environments. To stress the difficulties of this, consider trying to create for example, a form with projections as seen in Figure 48. You can actively try to build it as was done in the physical model, with gravity and forces actively simulated, stacking elements through trial and error and hoping it does not collapse with each successive move, or elements are approximately placed, "floated in space", not unlike current modeling methods and then the hope is that it stands once forces are engaged. Here, drawing a building amounts to trying to building the drawing.

Granular behaviour requires physical, empirical testing, but digital computation and simulation becomes a necessary method for testing and is no longer restricted to scientists and engineers. These tools will be indispensable in allowing for analysis and better understanding of the material behaviour. This discussion surfaces a major challenge/ contradiction of how to represent self-organizing and random form - to determining the indeterminate. Even with a successful simulation generated of an idea, its execution can differ quite drastically due to its sensitivity to a number of factors that may not be accounted for in the simulation.

\section{(Refer to Appendix A for additional photo documentation and an organizational chart of experiments and variables.)}

\section{Architectural Material}

The complexity of adaptability is systematically decomposed here physically in its material into smaller manageable problems with granular material technology. The tests conducted here are a general attempt to demonstrate the critical relationship between local element and global formation. Small, but precise changes of components were found to can have large impacts on overall form and behaviour. Stability and malleability can be achieved with specifically designed particles to produce forms not capable with natural granular materials yet still assuming its fundamental granular characteristics. Aggregation processes and methods play a vital role in the material's formation. Yet, a large component underlying all of this is the material's agency — its ability to compute its own structural responses. Various methods were explored keeping in mind scalability and access. In the context of an adaptable architecture, as a material system it affords the ability to be changed and transformed, built and rebuilt, while displaying resilience. 


\section{Projects}

What follows are attempts to exploit the strengths found in designed granular material for an adaptive architecture. Three different, but related applications constitute the main project of this thesis. The first is an unbuilt Sukkah design created as a competition entry. It is the culmination of the procedural investigation just discussed and an attempt at addressing architectural themes. A second project called Flow was documented as it was built and rebuilt showing the process and factors involved in realizing a full-scale granular structure. Lastly, the garden project Seeds is an adaptation and repurpose of Flow reusing its components. 


\section{Design Exploration 1: Sands of Tomorrow — Adaptive Architecture Studio to Sukkah}

May-June 2015

An architectural competition for a temporary shelter (a Sukkah) provided a general architectural program that framed the development of a habitable enclosure. The competition design guidelines called for innovative structure that delicately balanced the themes of new/old, open/closed and temporary/ permanent to be built at Toronto's Nathan Phillips Square (Sukkahville 2015).

Architecturally, the material system worked well to address the themes of the competition brief. A re-imagination of the Sukkah (a temporary hut constructed for use during the Jewish festival of Sukkot) was the objective. With the theme of temporality central to the competition, a simple hut was proposed, capitalizing on the loose method of assembly afforded by the novel aggregate system. The proposal would acknowledge the theme of new/old by combining new material with traditional concepts of building. The historic roots of the Sukkah were also recognized with the system speaking to aspects of resilience and the desert. A protective enclosure was generated while the rough agglomeration of material created a porous environmental mediator of light and air that addressed the dichotomy of open/closed. The stunning visual effects produced by filtering sunlight, creates a rough, cavernous, spatial quality, but a sense of warmth is achieved with wood as the material (Figure 63). Finally, the idea of permanence was hinted at on different levels. The components, operating within a continuous cycle of reuse—made possible due to its multiple possible interpretations and simple material—becomes enduring, while the lattice structure, if left to itself can have a lasting function, becoming a permanent host for future flora and fauna due to its ability to accumulate materials (Figure 64).

By attempting to apply the research through an architectural project/ program, it began to move beyond matters of performance and engage issues of process from a design methodology and a constructional front. It revealed challenges in terms of the design process, control, planning, and representation. Construction issues including safety, material use, and sequencing were considered. Its relatively simple geometry leaves room for the imagination to interpret, discover, and appropriate - ideal properties to adapt to the greatest variety of needs.

(Refer to Appendix B for additional images.) 


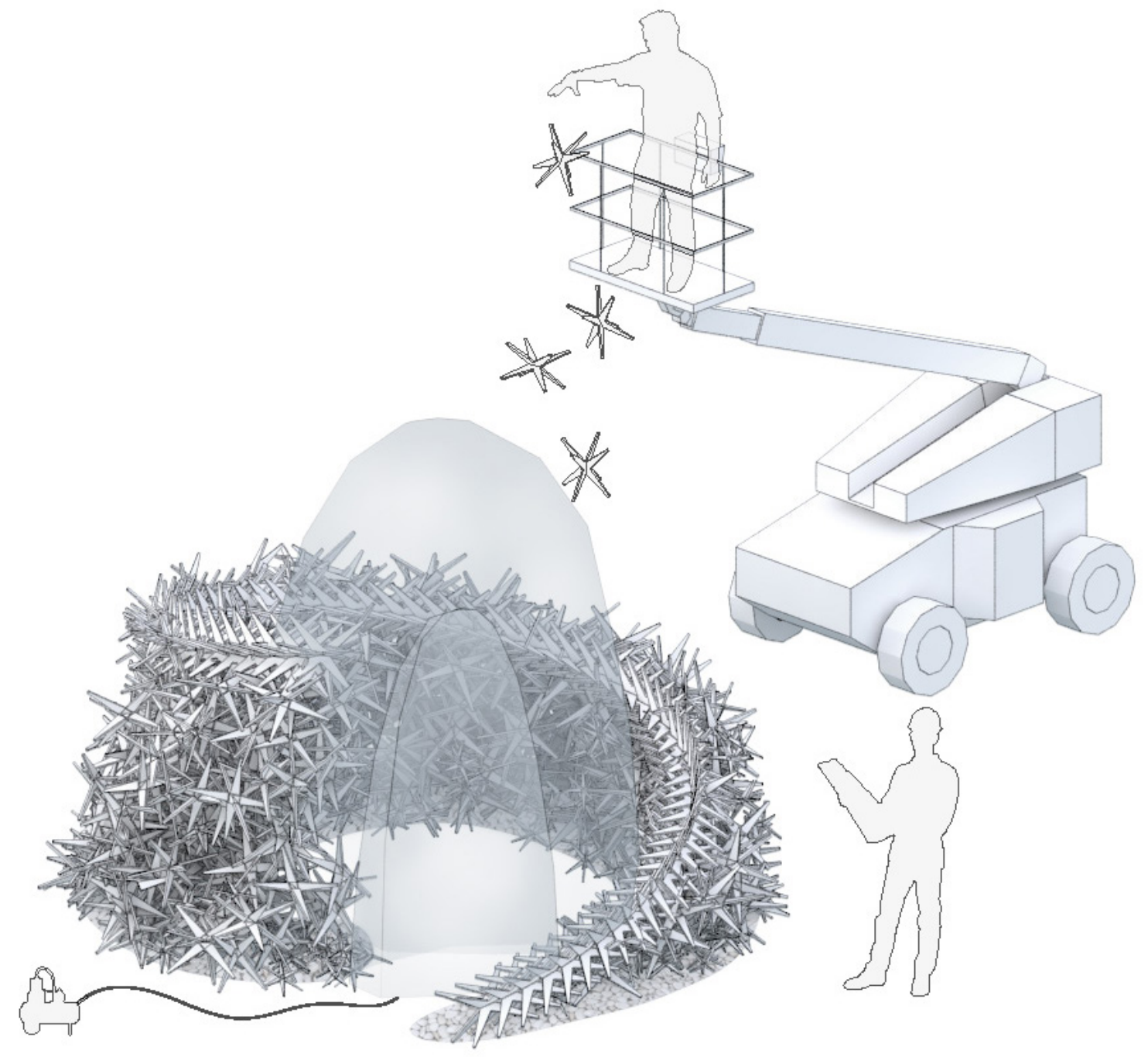

Figure 62. Construction concept. 


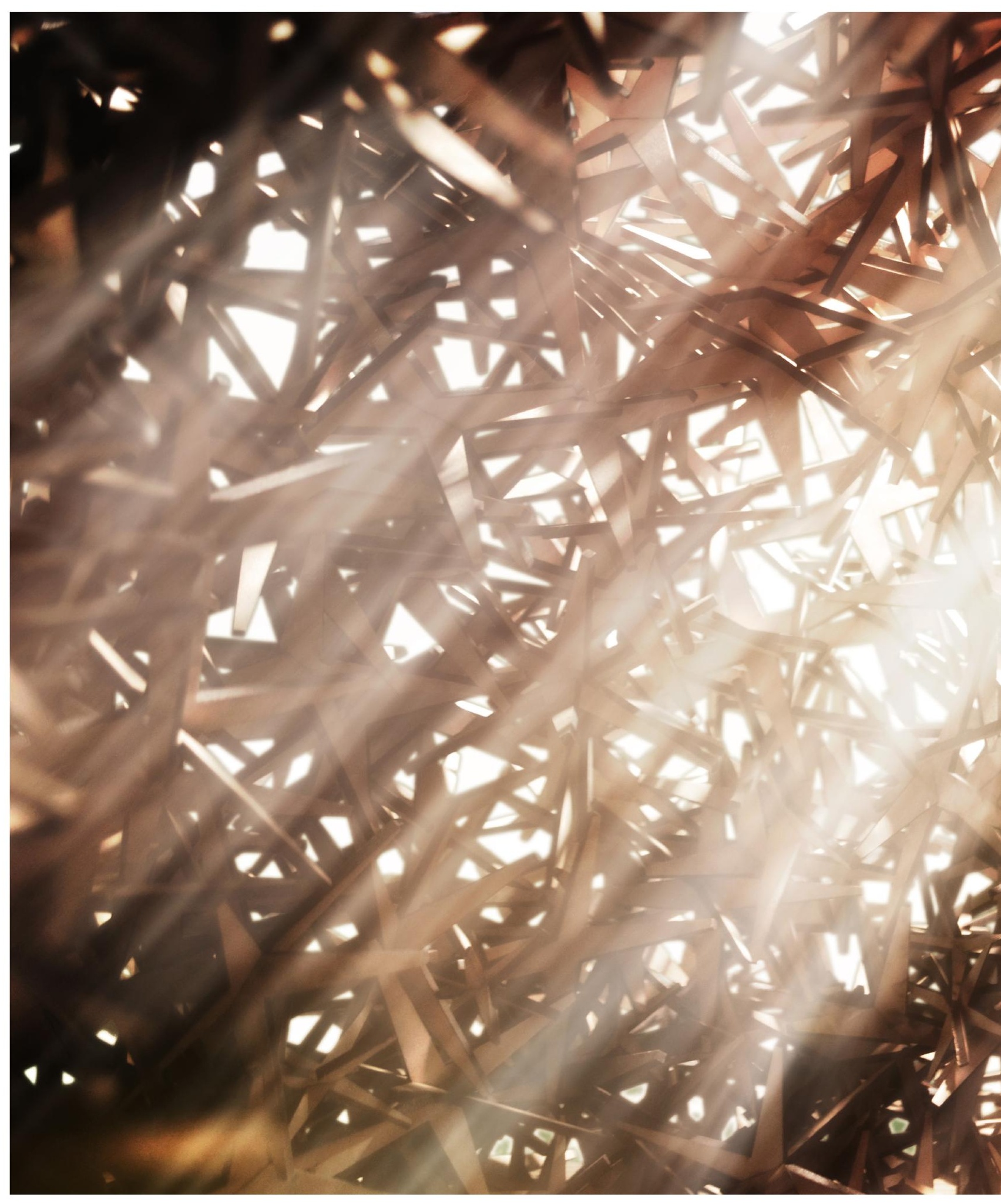

Figure 63. Spatial qualities from interior 


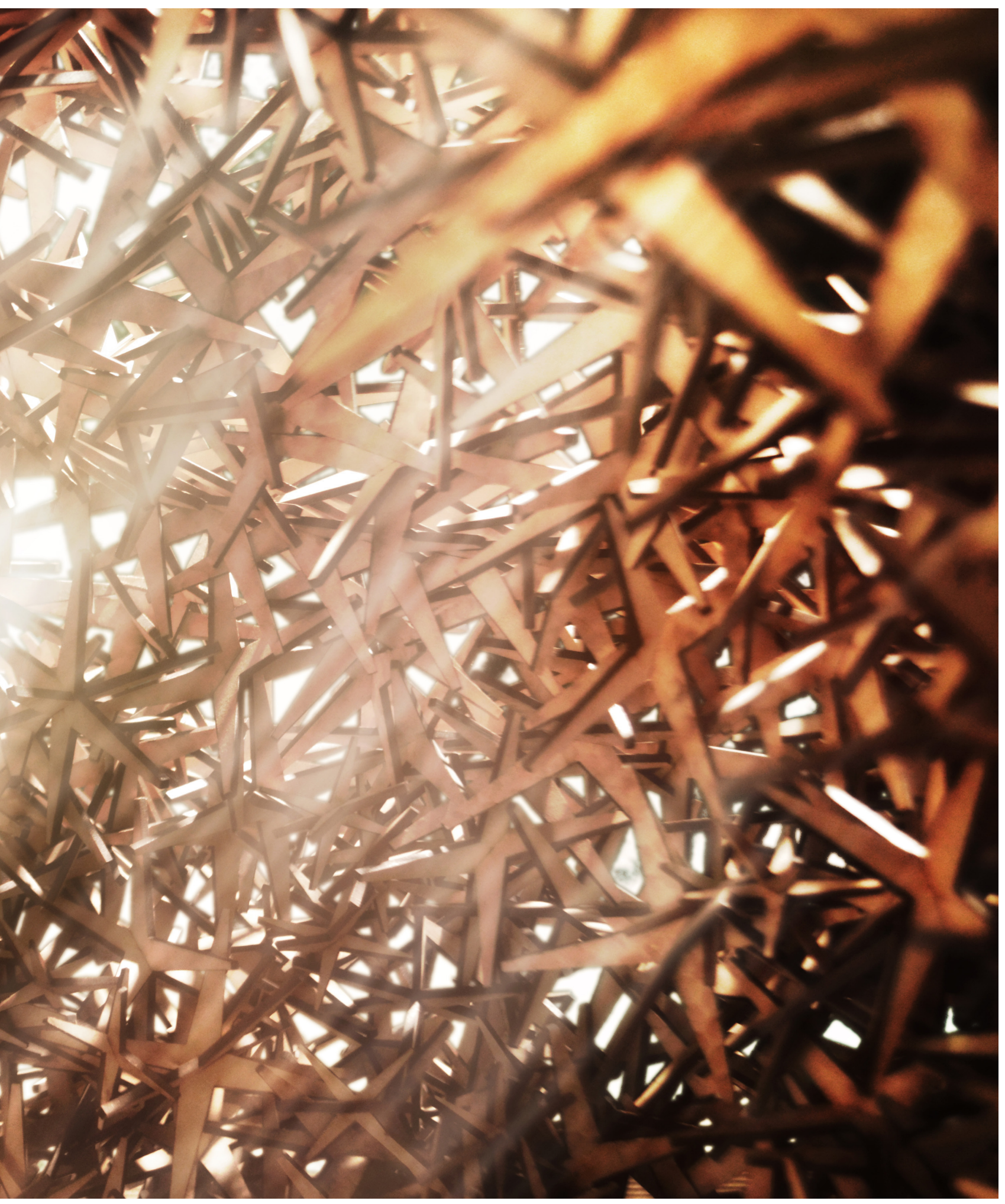




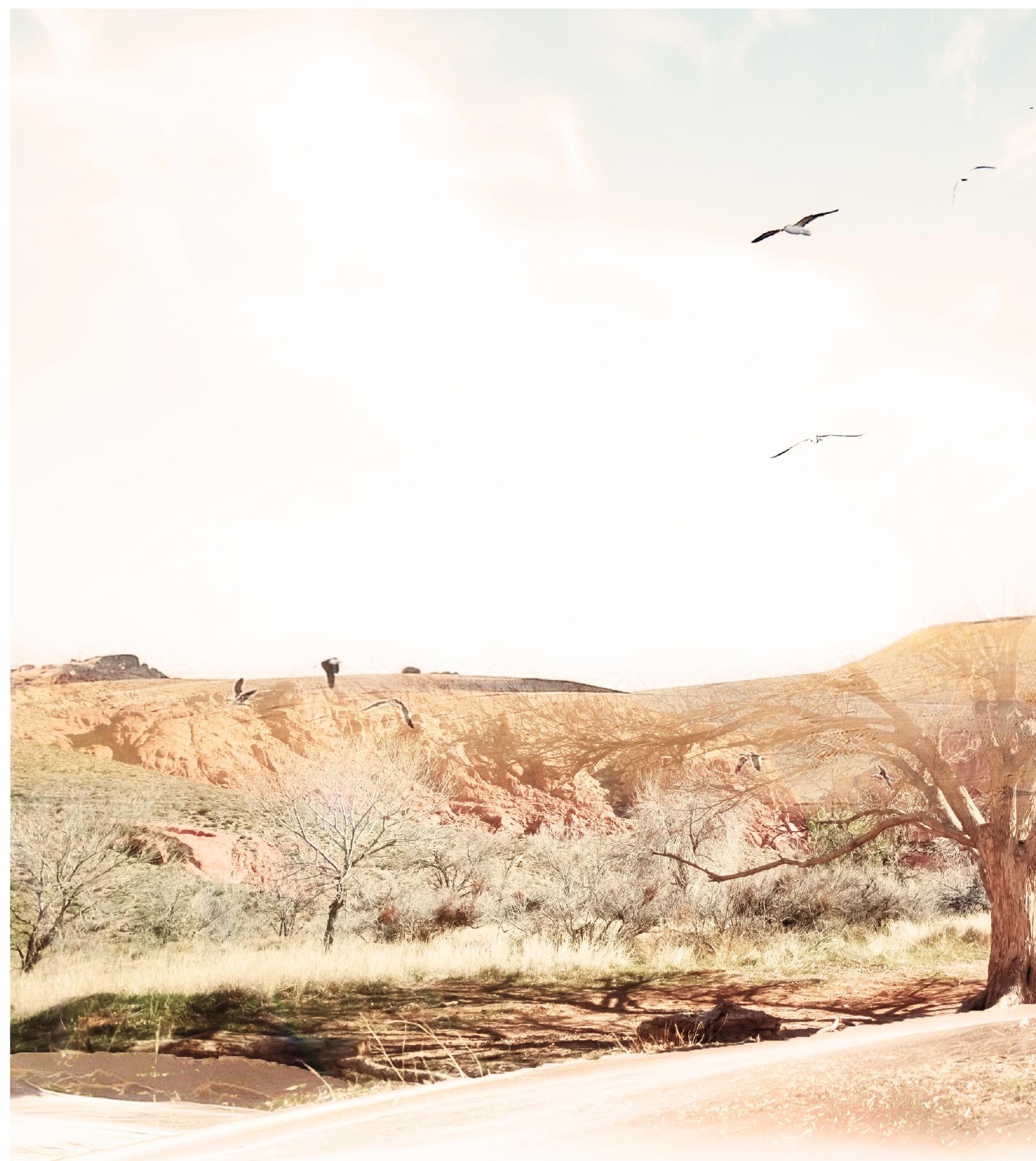



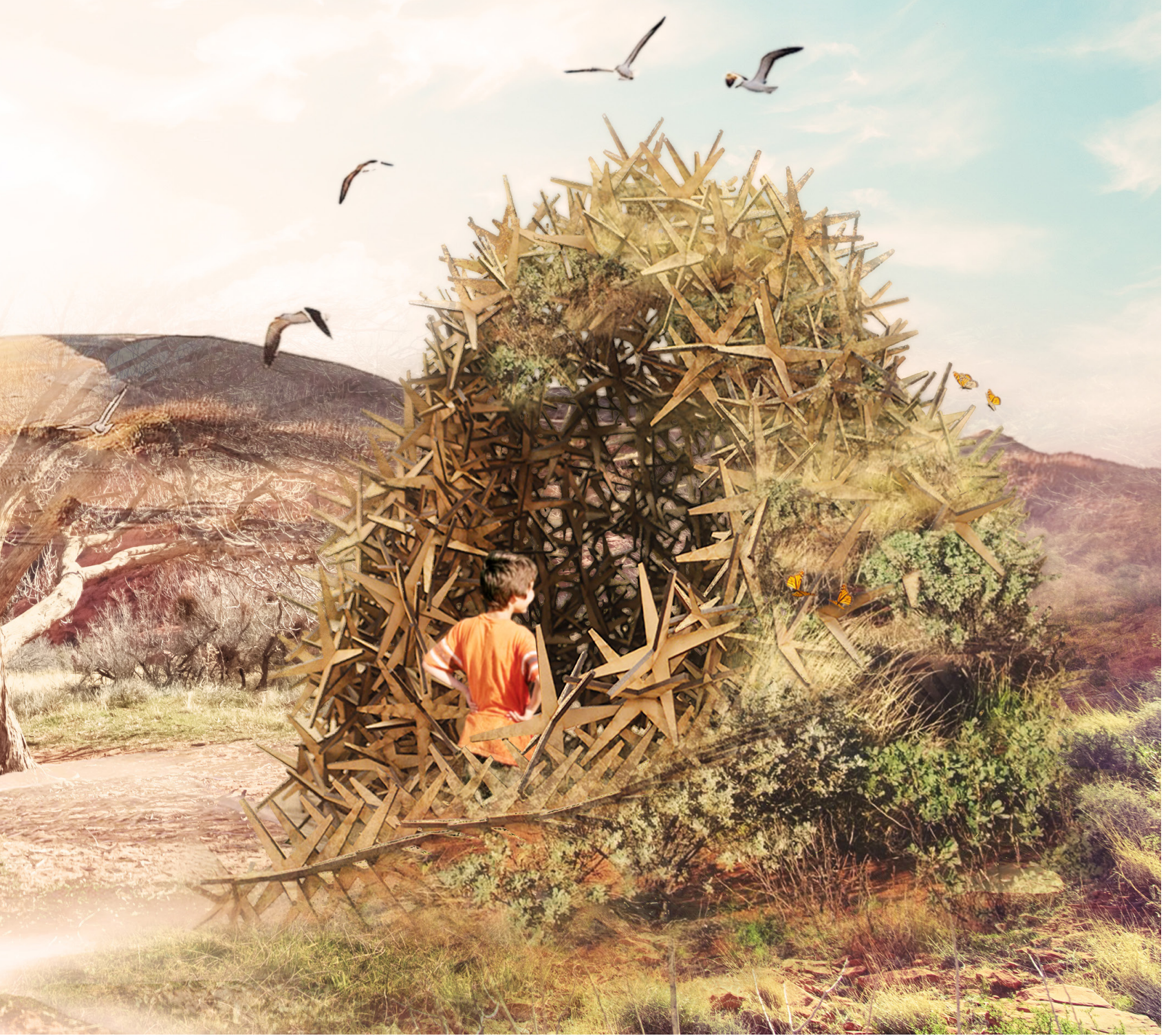


\section{Design Exploration 2: Flow (Winter Stations 2016)}

February - March 2016

\section{Competition Background}

Toronto winters are long and unpredictable. They consist of varying temperatures, amounts of precipitation, wind, in short: dispositions. There are days the snow falls heavy. Other days are deceptively bright and sunny, but with bristling winds and plummeting cold. The snow accumulates, along with the ice, and sometimes it is blown away, dispersed in drifts. But at some point during the long winter months we wait for the temperatures to rise and the sun to melt it all away. Whether it will do so for good is always an unknown.

The theme of Freeze / Thaw asks designers and artists to respond to the changing climatic conditions and transitions of the Toronto winter. Designs may anticipate the coming spring or refuse to yield, reminding us December is only a few months behind and will return again. They may highlight a static moment or phase in our winter. Perhaps, most significantly, designs have the opportunity to observe, reflect or contrast the immediate waterfront landscape with its banks of snow and frozen ice. To this end, they should expect to be playful and provocative, creatively utilizing materials and site to inform concepts that echo the freeze / thaw narrative and will engage the public.

(“Winter Stations Design Competition 2016,” 2015)

\section{Objectives}

The themes and requirements of the competition aligned closely with the properties of granular material systems. Flow was selected as one of four international projects and built at the beaches for the months of February and March. Its selection allowed the advancement of a full scale prototype and test some of the assumptions from the previous design exploration regarding realization, construction technique and processing.

The requirements of this brief presented a much simpler task than the Sukkahville competition as it did not specifically specify the need for a shelter. Furthermore, it was an opportunity to engage a context of great uncertainty. 


\section{Concept}

Flow attempts to capture the transitional moment between freeze and thaw through the reinterpretation of a single ice crystal. The elementary particle is reinterpreted as a 3D star-shaped module digitally fabricated with slot-fit wood connections. Informed by aggregation processes exhibited by natural granular material such as snow, the system contains over 1000 modules and is capable of crystallizing into a meta-stable, solid state; yet the material is able to be easily reconfigured, like a liquid, due to the system's loose bonds. While snow melts away eventually leaving little trace behind, Flow serves as a reminder of the snow structures that once speckled the landscape. The dynamic process of freezing and thawing, like water transforming from solid to liquid, is expressed in an equally charged wave-like form capturing the flow of energy (Figure 65). (Description submitted to the Winter Station competition.) 


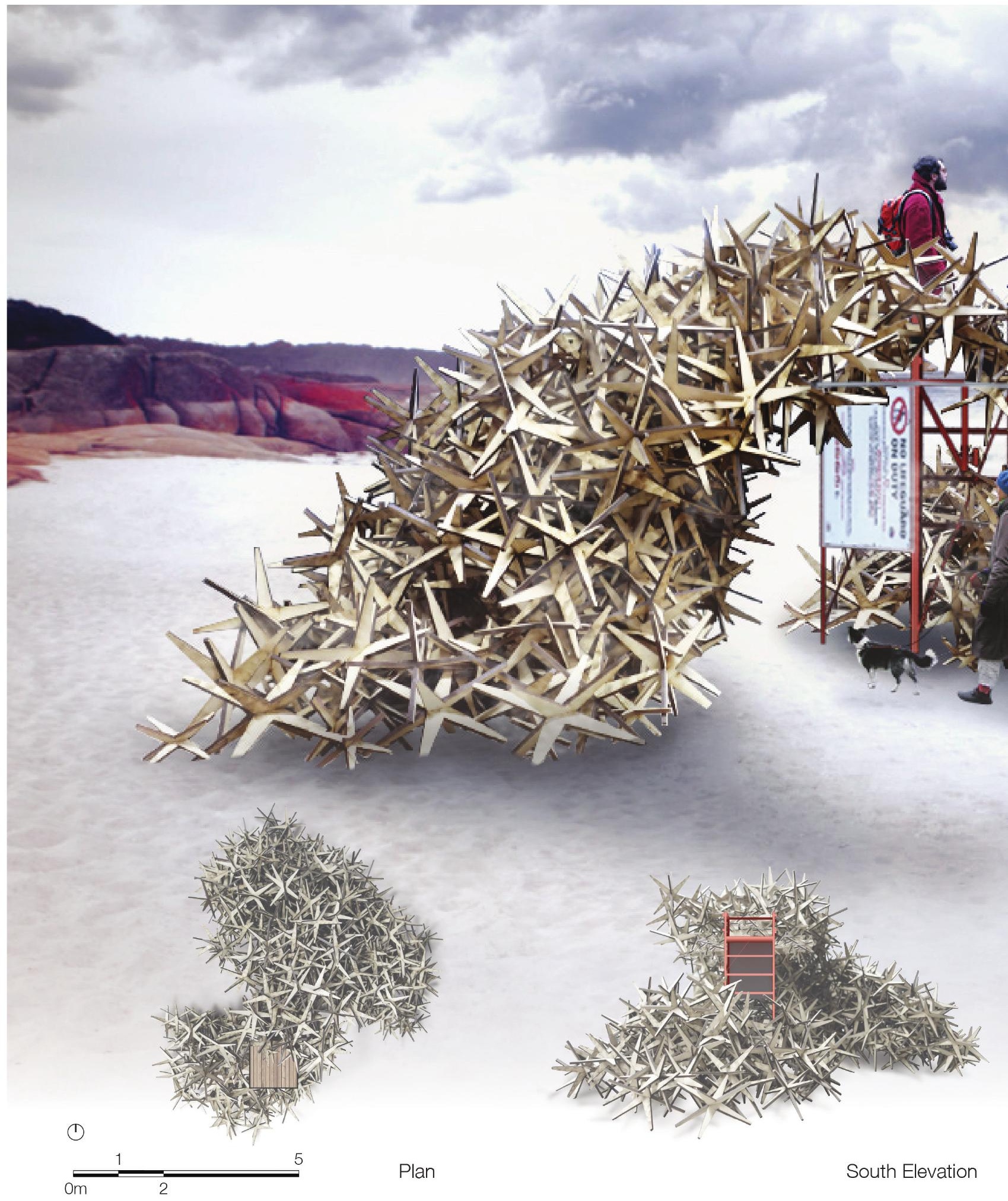

Figure 65. Flow Winter Station proposal 


\section{Coordination}

Consideration and thought had been given during the previous design exploration on the factors that would affect construction and realization of a full scale structure. A key difference and a valuable condition introduced in this scenario is the involvement of external fabricators, rather than fabricating the components ourselves. As expected, but important to note, was that the coordination required was minimal. One email was all that was exchanged between the fabricators and us to get the project started. The email included the CAD file with the components laid out on a 4 foot by 8 foot sheet, the number of units required and the material requirements. Although the fabricators made a number of changes without our consultation - in material quality and thickness - the general performance, behaviour, and intention of the design remained. This speaks to the material system's flexibility to adapt to available materials and conditions.

\section{Fabrication}

The fabricators used 5/8 inch construction grade plywood which is slightly thicker and rougher than the $1 / 2$ inch thick, birch plywood prototypes constructed previously. The pieces were much more rugged, a factor related to how the material responds to being cut using a CNC, and also the pieces were not finished (sanded). A number of particles were spray painted blue for aesthetics, but also provided a useful visual cue to judge depth and helped the form to be read. A key difference between the two fabrication technologies is that it is difficult, and not the best practice to have particle profiles share edges as could be done in laser cutting to reduce the number of cuts. This leads to an increase in fabrication time. In total about 1,300 units were created. The number of units required was estimated by projecting from the quantities used from the more reliable physical scale model rather than rudimentary digital models where they are still far from being resolved. This poses a challenge for design when considerable quantities may be required and physical modeling becomes unfeasible.

To maximize the number of units that can be fabricated within the budget and to limit waste, efficiency in material use took on greater importance. As found previously, using two sizes of particles has the advantage of creating a more robust system when combined, but it is also a more effective use of materials. However, when strictly looking at the yield in terms of the sheet layout, it is capable of utilizing merely $27-32 \%$ of the sheet material (Figure 66). Using an algorithm to nest the pieces yields similar results. It may be possible to nest and with the addition of an even smaller particle to increase utilization. The efficiency is greatly attributed to the nature of the arms. Shorter arms produces greater packing. Another possible fabrication method considered was to create the profile from a linear element and take slices from it. 

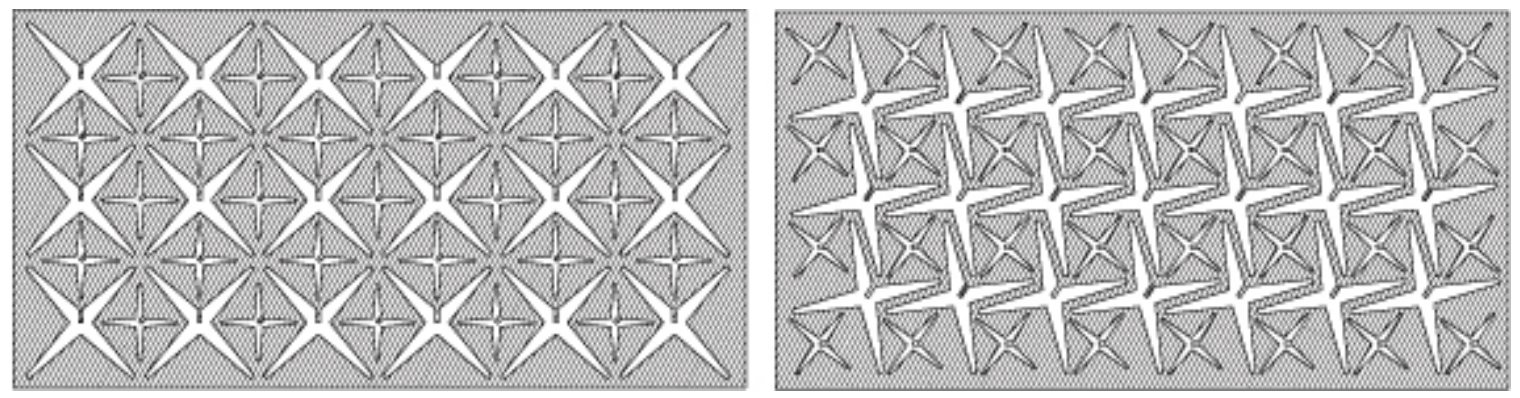

Figure 66. Layout of 4'x8' sheet material for CNC milling. 27.8\% effciency (left) 32.5\% efficiency (right)

\section{Security}

Based on previous string experiments, a binding system using ropes was purposed to act as a redundancy in case of structural failure. The initial idea was to envelope the whole structure in a loose web. In this scenario it would have been important that the rope was not actively compressing the material together and the system was allowed to settle as it required to avoid the introduction of active stresses. The ropes would catch the pieces should the structure begin to fail. The idea evolved into a more discrete solution, concentrating the rope system on a critical area that would be masked with free pieces. It was reasoned that since the components were all part of an interconnected network, a stable "core" should provide sufficient security for the system.

A wood lattice was suggested as an alternative by the fabricators, where an arched lattice could have provided the framework on which the material can aggregate. It was ruled that the lattice would very likely force an unnatural arch to be created increasing, its chance of failure. It would also diminish the structural capabilities and overall effect and design intention. This was not pursued.

\section{Mock-up}

A test build was conducted at the fabricator's shop where a sizable amount of material was created (Figure 67). Valuable insights were gained. The roughness of the pieces, although made it difficult to handle, added friction and contributed to the stability of the structure. It was observed that the smaller components played a much greater structural role than previously anticipated. Not only would the smaller components create local modulations in density, but they behaved like a binding agent that would fill in between the larger components. Alternating between large and small components during placement appeared to make a much more secure structure. 
From the initial testing, a semi-controlled process was necessary to work with the material (when the material is deposited one component at a time). It is a process that still requires monitoring and some judgement and as such like a carpenter or mason who develops an "understanding" of the material they work with, a similar "feel" can be developed for this material despite the seemingly chaotic and unpredictable outcome. Design is conducted in real-time via feedback from observation. Actual knowledge of the system here, with all its variables is acquired through doing. Still, the skill required is relatively low, it is very accessible requiring few to no tools to assemble and the system is quite forgiving. That is, the network of particles form a decentralized system which allow it to be very robust.

\section{Observations}

During both the test build and the final build a partial collapse occurred while creating the arched shape. Collapse could be accelerated and attributed to human bias while depositing the material. Whether consciously or unconsciously done, an unbalanced distribution of material would lead to collapse. Simply where one is standing or where the source material is located in relation to the structure has an effect and would start to bias a certain portion of the structure and neglect other areas based on accessibility. The collapse then, it was concluded, is actually an important and beneficial process. It mitigates the human bias, by resetting the system to an optimal condition, allowing subsequent moves to build off a stronger state. A new order emerges from the collapse, the reorganization allows for a more complex structure. This aligns with the observation of criticality in sand piles. An avalanche event makes the system more stable reducing the energy for larger, catastrophic avalanches. The edge of chaos affords stability through resilience. Alternatively, a process of periodic agitation/ vibration would allow self-organization to occur and thus the structure can self-heal and strengthen. This was verified empirically in controlled tests with the smaller scale system as discussed.

It should be noted that while much was learned about the material system in general from the test build, it was limited by the fact that no two deployments would ever be alike as the system accepts external pressures from its unique environment to take shape. Especially in this case, the difference between the calm, indoor setting of the shop greatly differs from the dynamics of the outdoor beach environment. In a chaotic system such as this, even the slightest changes in initial conditions can mean the difference between stability or collapse.

The most challenging part of the process it was anticipated, was to be with transport and storage at the conclusion of the event. The pieces, being so voluminous, would become cumbersome to move. Disassembly of the components was then considered. Due to time constraints and the fact that they would need to be immediately reassembled, the decision to implement the modifications were ultimately ruled out. This issue however still initiated a greater investigation in particle joinery for disassembly. 


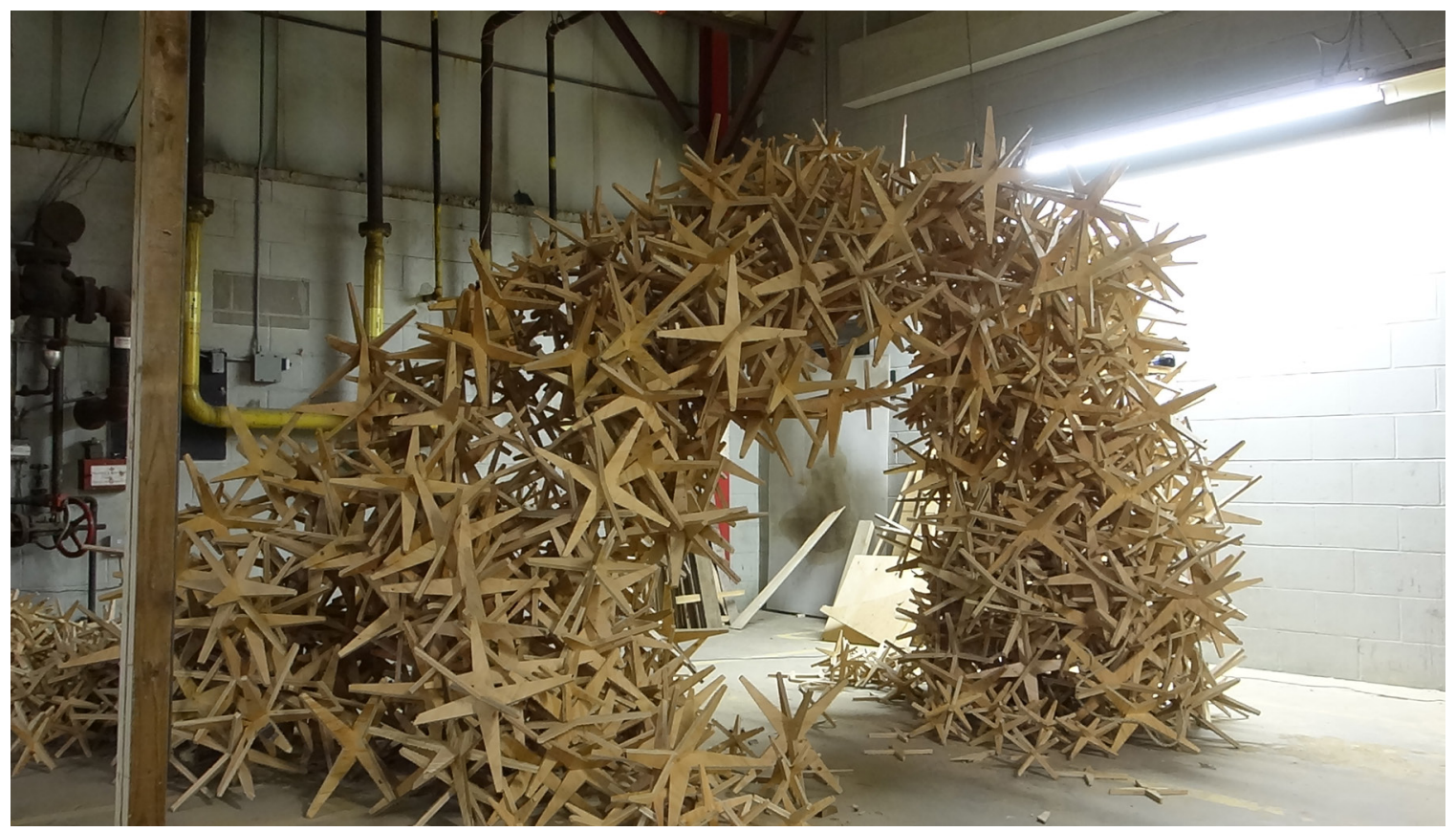

Figure 67. Flow test build in the fabricator's shop 


\section{Particle Joinery}

\section{$\underline{\text { Slot Joint }}$}

The friction fit, slot connection is valued for its simplicity, but it has some limitations. The slot must be precise - too tight and it becomes difficult to put together or take apart and too wide it becomes loose and the pieces slip apart. Variations in material thicknesses would also need to be considered and require calibration for each sheet. Secondly, constant assembly/disassembly will eventually wear out the connection and the pieces will not hold together. In anticipating the challenge of transporting and storage of the components after the event, the connection of the individual pieces was identified as a critical area of investigation that led to the search for alternative forms of joinery.

$\underline{\text { Dowel pin }}$

The dowel pin also uses friction and acts to wedge in between the two plates bridging the gap in the slot (Figure 68). The pin, a standard woodworking accessory, could be taken out or replaced to free or renew connection. It however, would require a tool to drive in the pin, and the pin could be prone to loss.

\section{$\underline{\text { Dovetail joint }}$}

A dovetail joint uses geometry to interlock pieces together. It requires an elaborate process involving great precision and thus much more time to fabricate. It also distinguishes the once identical plates into male and female counterparts that requires its own processes and recognition during assembly.

\section{Shock cord}

The development of the shock cord connected module was derived from modern tent poles which are linked by a cord. The slot is augmented with an elastic shock cord threaded through the two plates (Figure 69). A precise, tight fit is no longer as critical and as such makes assembly and disassembly much easier without concern over the joint wearing. The tension of the cord serves to keep the pieces together and allow the constituent parts to remain connected together while dissembled. Although this solution was not implemented in time for the Winter Station, it overcomes many of the aforementioned issues surrounding assembly. By allowing the component to return to a flat state much more easily, greater ease in transportation and storage can be achieved. Importantly, this enhancement improved upon the simplicity of the original design. 


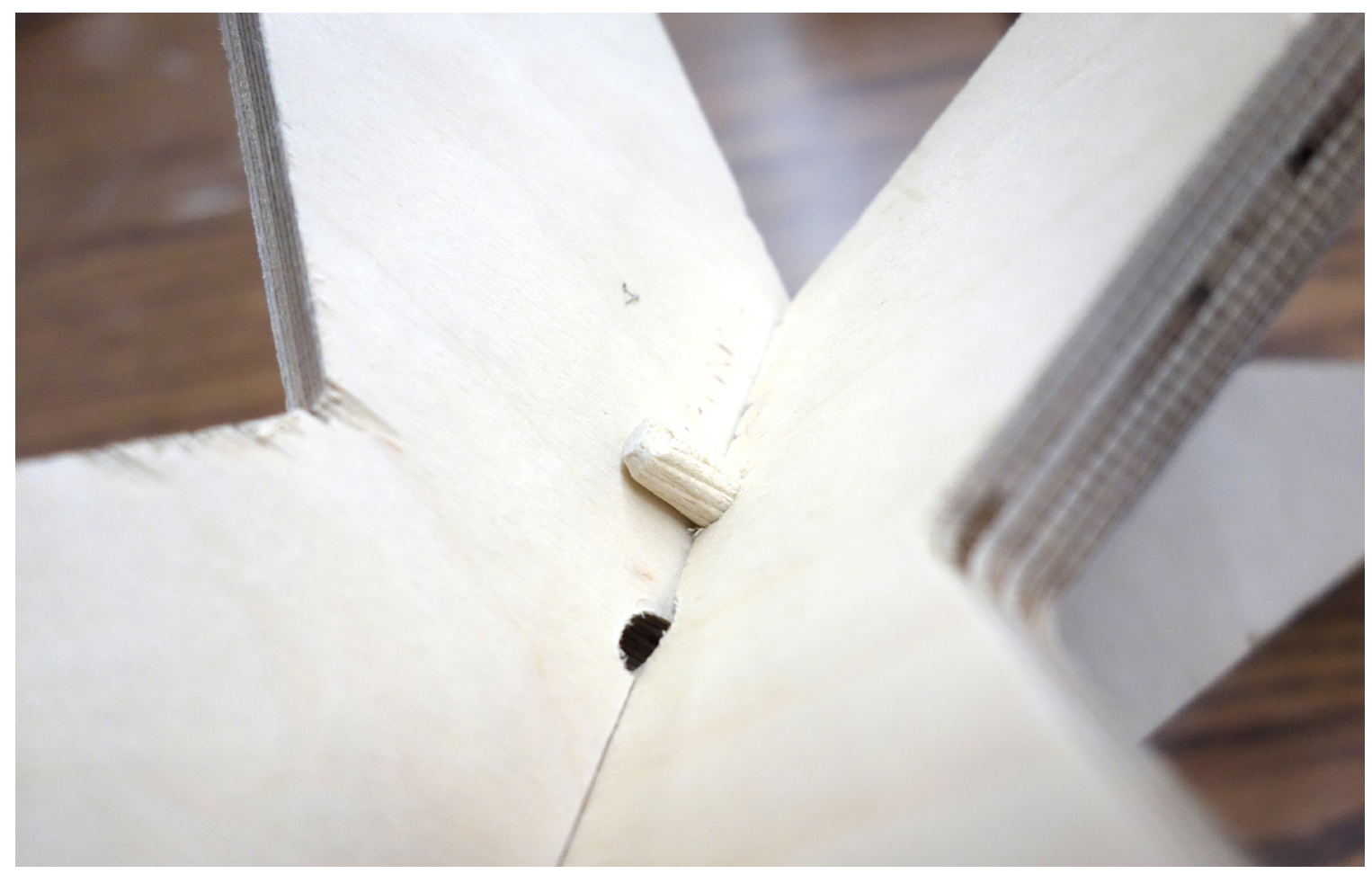

Figure 68. Dowel pin connection

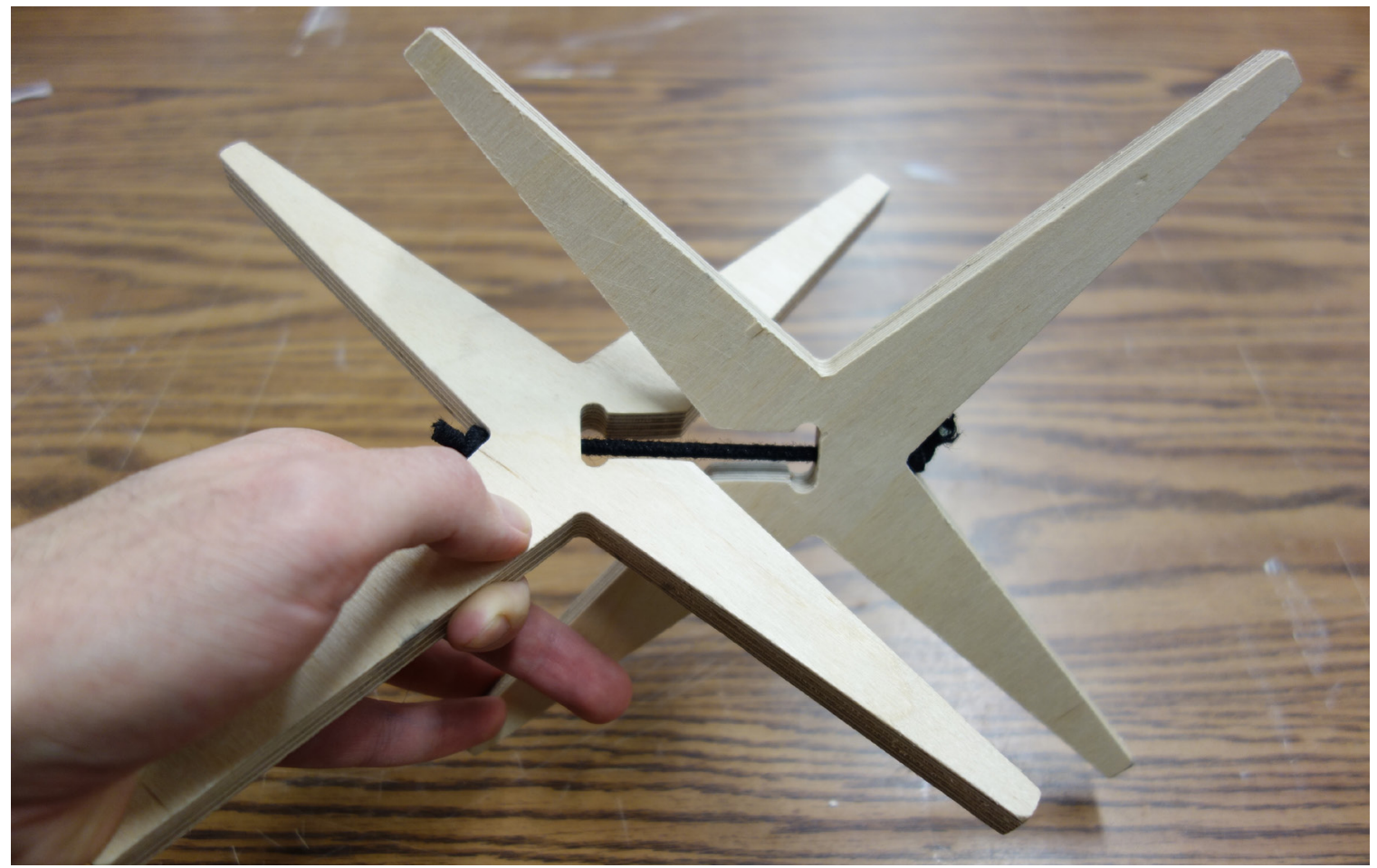

Figure 69. Shock cord connection 


\section{Realization}

The build took place on February 12 at Toronto's Woodbine Beach (Figure 70). It was an exceptionally cold day (high of $-4.5^{\circ} \mathrm{C}$ ) with high winds (reaching $44 \mathrm{~km} / \mathrm{h}$ ). Conditions were exacerbated due to its location by the lake. Wind chill made it feel like $-14^{\circ} \mathrm{C}$ (Environment Canada, n.d.-c). The components were already transported to the location by the fabricators. A frame and net, the width of the lifeguard stand, was extended out from the stand about one metre to provide something for the components to catch onto as it passes overhead (Figure 71).

The beach setting was very appropriate which allowed for the juxtaposition of the design granular material with a natural granular system. The sand worked well with the particle geometry allowing the particle to sink partially into the ground and take a relatively secure footing. As mentioned, this is important to increase stability of the structure and the sand could not have been a more desirable ground condition. The unevenness of the ground was not an issue-the system was easily able to adapt to the terrain (Figure 72 ).

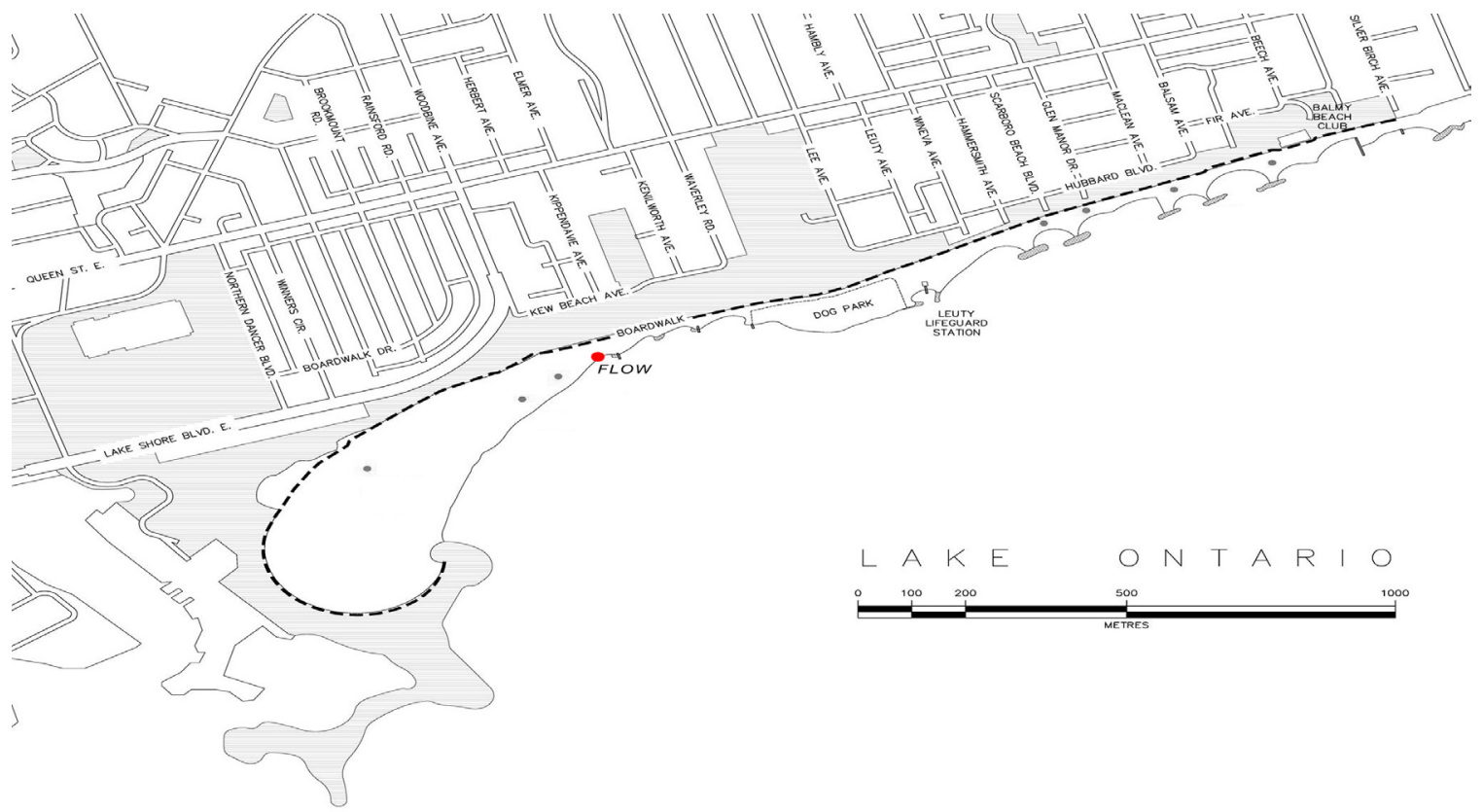

Figure 70. Location of Flow at Toronto's Woodbine Beach 


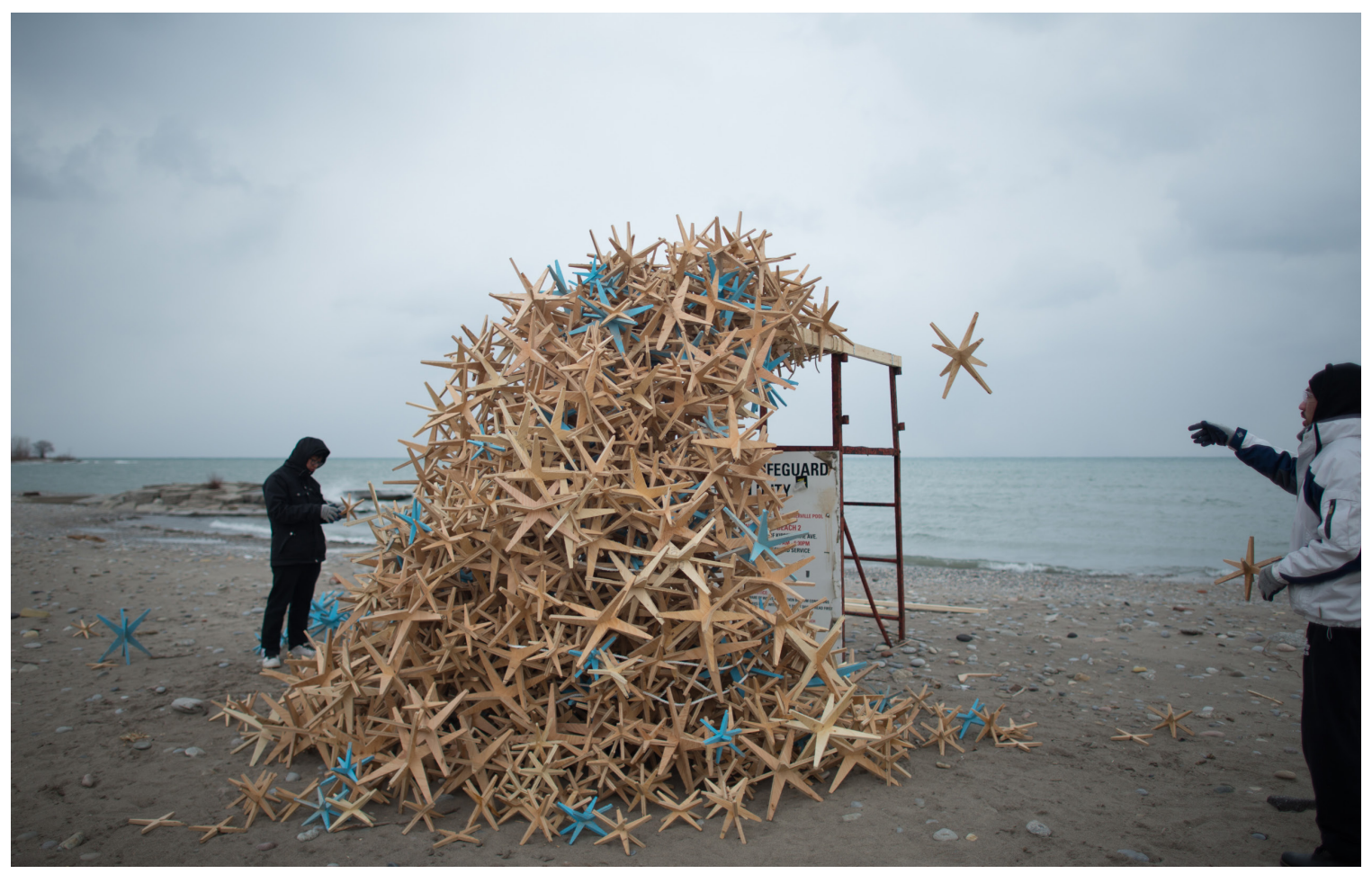

Figure 71. Installation under construction

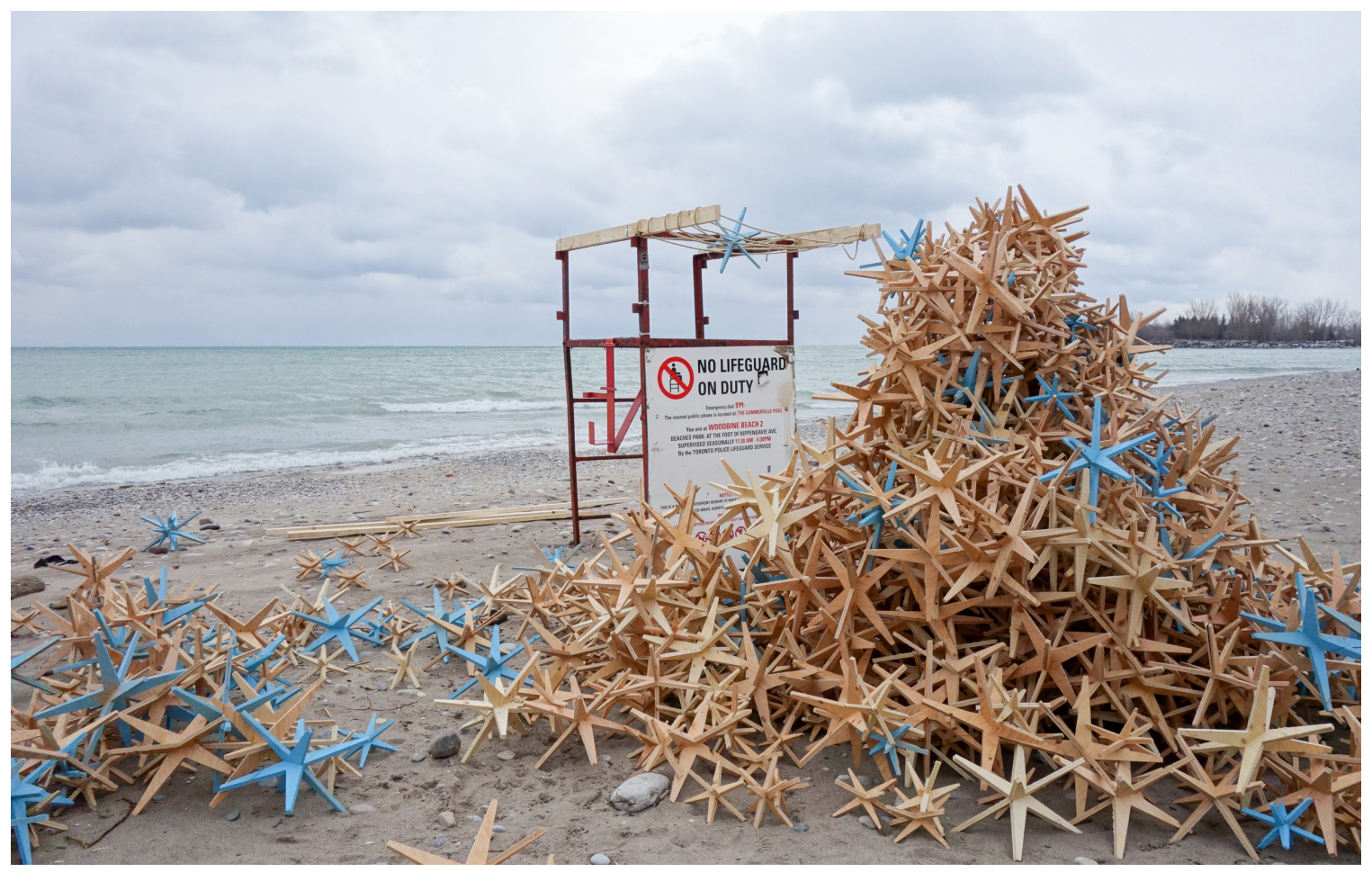

Figure 72. The beach setting was with sand and uneven terrain was desirable 
Wind became an immediate factor as the structure was being assembled. It was not that the structure could not with stand the winds, in fact, the structure held up very well. Without quantitative analysis it is assumed that porous quality mitigated any build-up of stresses. The challenge came during construction as the components would be carried by the wind, especially as the structure grew higher and it became harder to reach the top which necessitated the pieces be tossed together. Parallels of how the forces of wind and air similarly shape sand formations are seen.

The construction process took 3.5 hours for two people which is relatively modest compared to the other similarly scaled structures. If not because it was the first time, it could have been possible to complete the build even quicker. The process involved simply tossing the components together and then fine-tuning the form via adding and subtracting at specific areas. The process could be described as a form of sculpting and was not difficult and rather enjoyable. No plans were referenced during construction of the arch shape. In fact, in a spontaneous decision during the build, the design was changed to form a greater sense of enclosure by extending the width of the arch to form a quarter circle in plan (Figure 73). This capability is precisely what is desired of a truly adaptable system — to effortlessly accept uncertainty and respond to different conditions, environmental or human.

A rope binding system was used but quickly became quite hard to control at such a large scale and height, and was eventually abandoned leaving only a portion of the base bundled (Figure 74). The performance of the rope system thus was not properly evaluated and highlights that scaling technologies in practice is not always as simple. The granular system was scaled relatively easily.

\section{Adaptability}

The competition brief quite accurately described the uncertainty associated with the winter condition. The structure stood at the waterfront for 39 days (35 officially) and faced great variability. During this period, several weather records were broken in terms of both temperature highs and lows. Temperatures fluctuated between -26C to 20C (Environment Canada, n.d.-a) while the structure was exposed to ice, snow, rain and sunshine. During the course of the exhibition, visits were personally made on 15 different days under various conditions to document and observe the structure under changing conditions.

Although the structure was more an installation than a shelter, it had a perceivable ability to mitigate wind despite its porous nature. Despite the lack of any fasteners, it was able to endure winds that were reported to reached as high as $104 \mathrm{~km} / \mathrm{h}$. (Environment Canada, n.d.-a) Shifting sands caused several components to be found almost completely submerged in the sand, naturally establishing a stronger foothold in the ground (Figure 75). In addition to wind, the system was also tested by snow. At one point during the 39 days, it faced $9 \mathrm{~cm}$ of snow. (Environment Canada, n.d.-b). Loading from the snow did not seem to pose any structural challenges. The snow was seen to collect within the multitude of crevices created by the interlocking geometry filling in many of the openings and forming a less 
porous enclosure (Figure 76). Snow, as a natural granular material becomes a supplementary system that naturally complements the larger, synthetic granules to mediate the porosity and even perhaps provide insulating qualities (Figure 77). It can be imagined that with other forms of similar natural materials such as mud or clay it can be hybridized and enhance the properties of the granular system. The result of temperature fluctuations also caused a build-up of ice. It had the effect of binding some of the components together through freezing (Figure 78).

The weather also got very warm and under these conditions sunshine and light could be seen permeating the structure (Figure 79). Due to these dramatic changes in weather conditions however, the plywood components as expected did not hold up very well in some cases. Moisture getting in between the layers of wood caused layers to buckle and delaminate (Figure 80). The wood creates a sense of warmth, while the geometry and layering of components creates an interesting play of light and shadow (Figure 81).

The themes of the brief and the setting at the beach could not have worked better for the goals of this project. Thus far, Flow has shown an ability to cope with a great deal of environmental variability. That however, would not be the greatest source of uncertainty and challenge that the material would face, as it will be discussed in the next part.

(Refer to Appendix C for additional photos and documentation) 


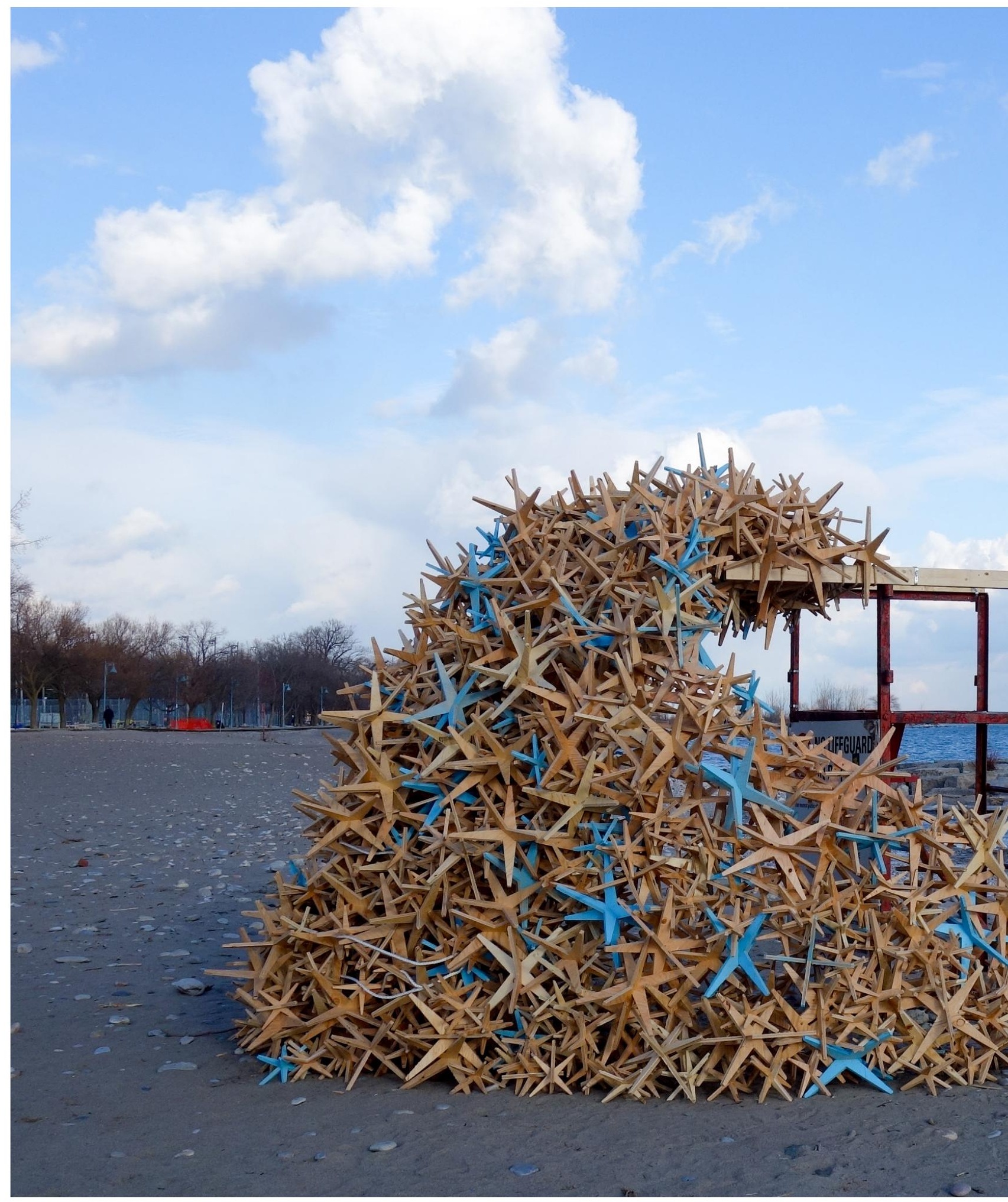

Figure 73. Flow newly constructed

114 Architecture at the Edge of Chaos 


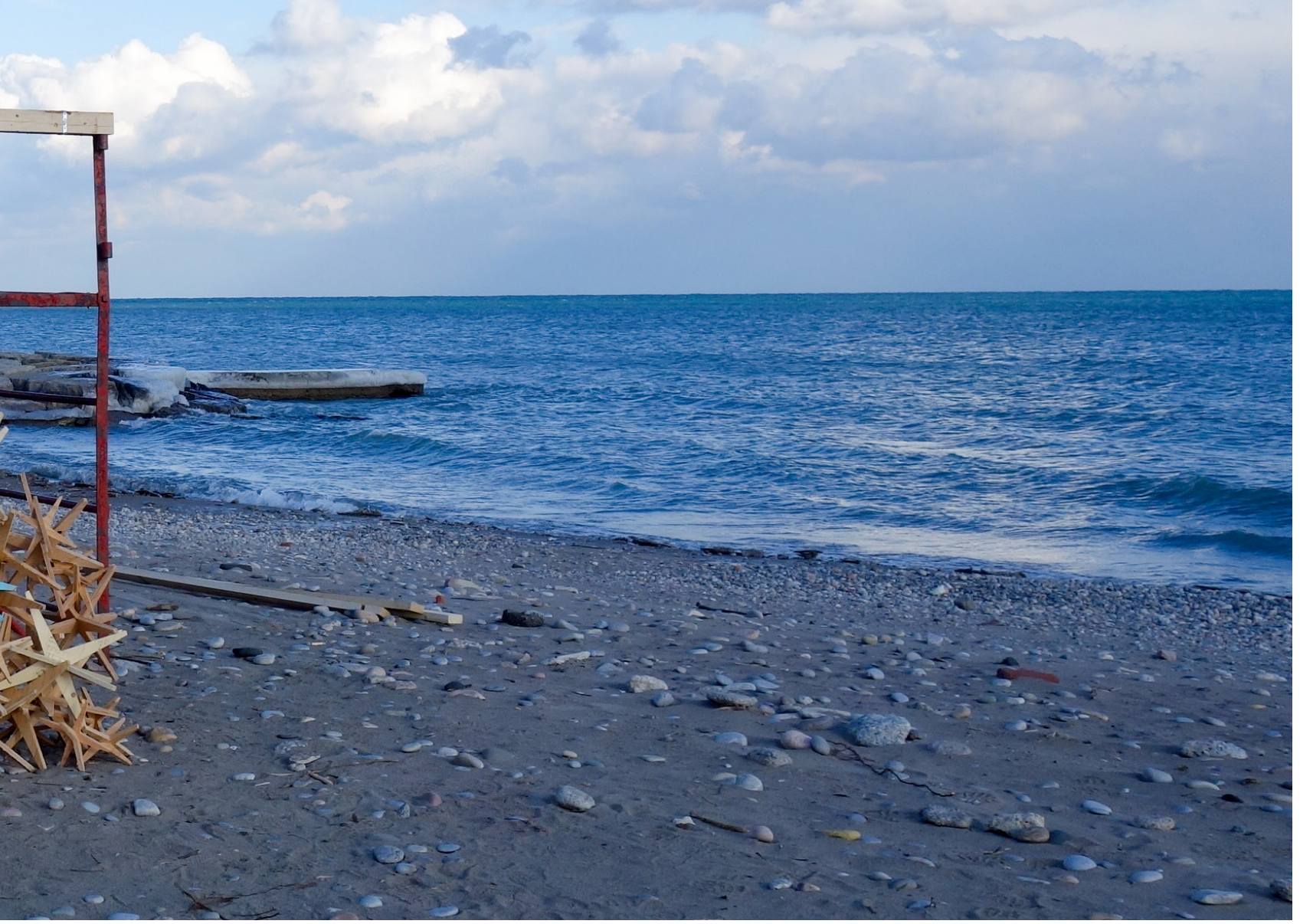




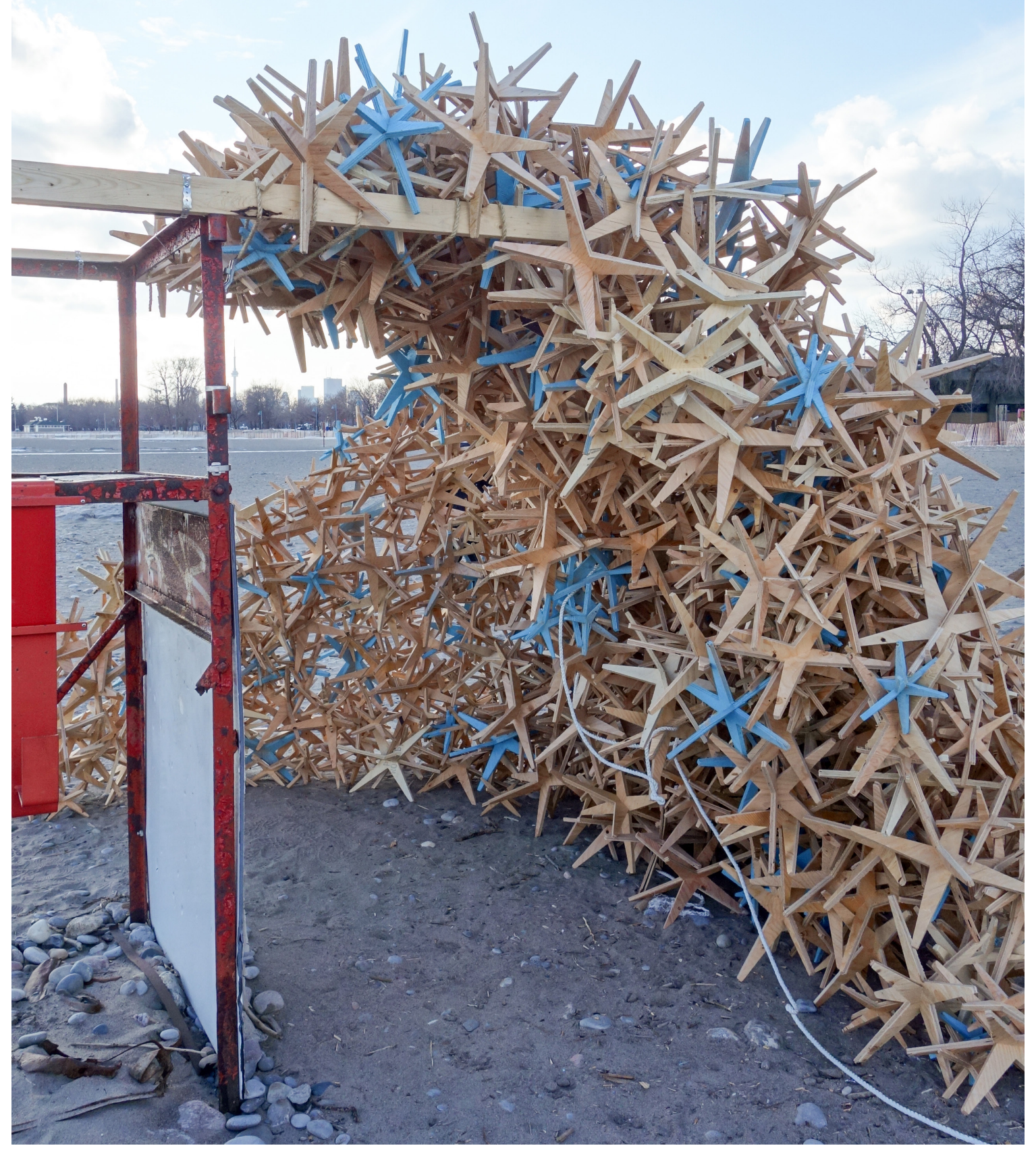

Figure 74. The rope binding system became too cumbersome to get around the form 


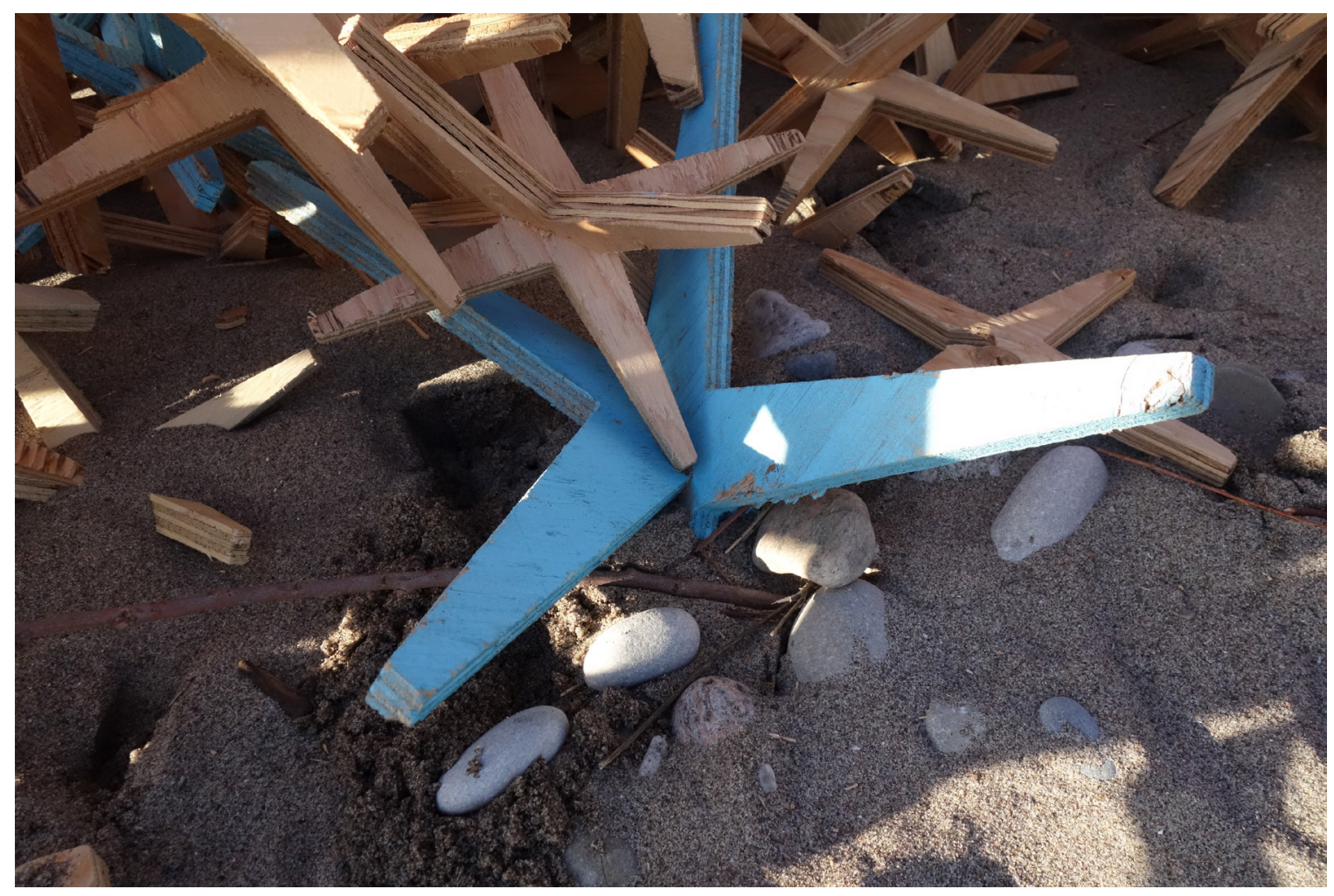

Figure 75. Particles naturally submerged into the sand provides a stable foundation 


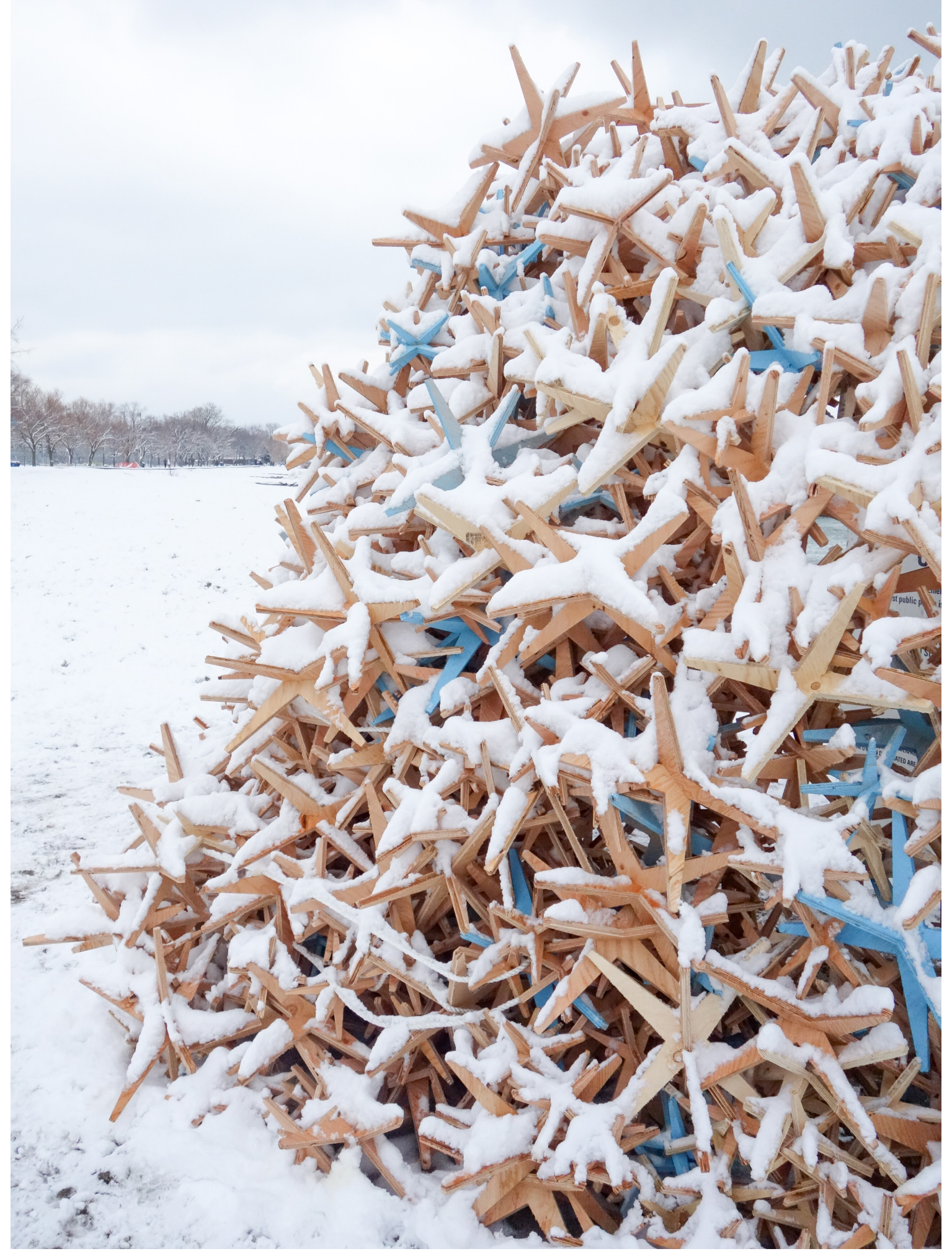

Figure 76. Snow accumulation on structure 


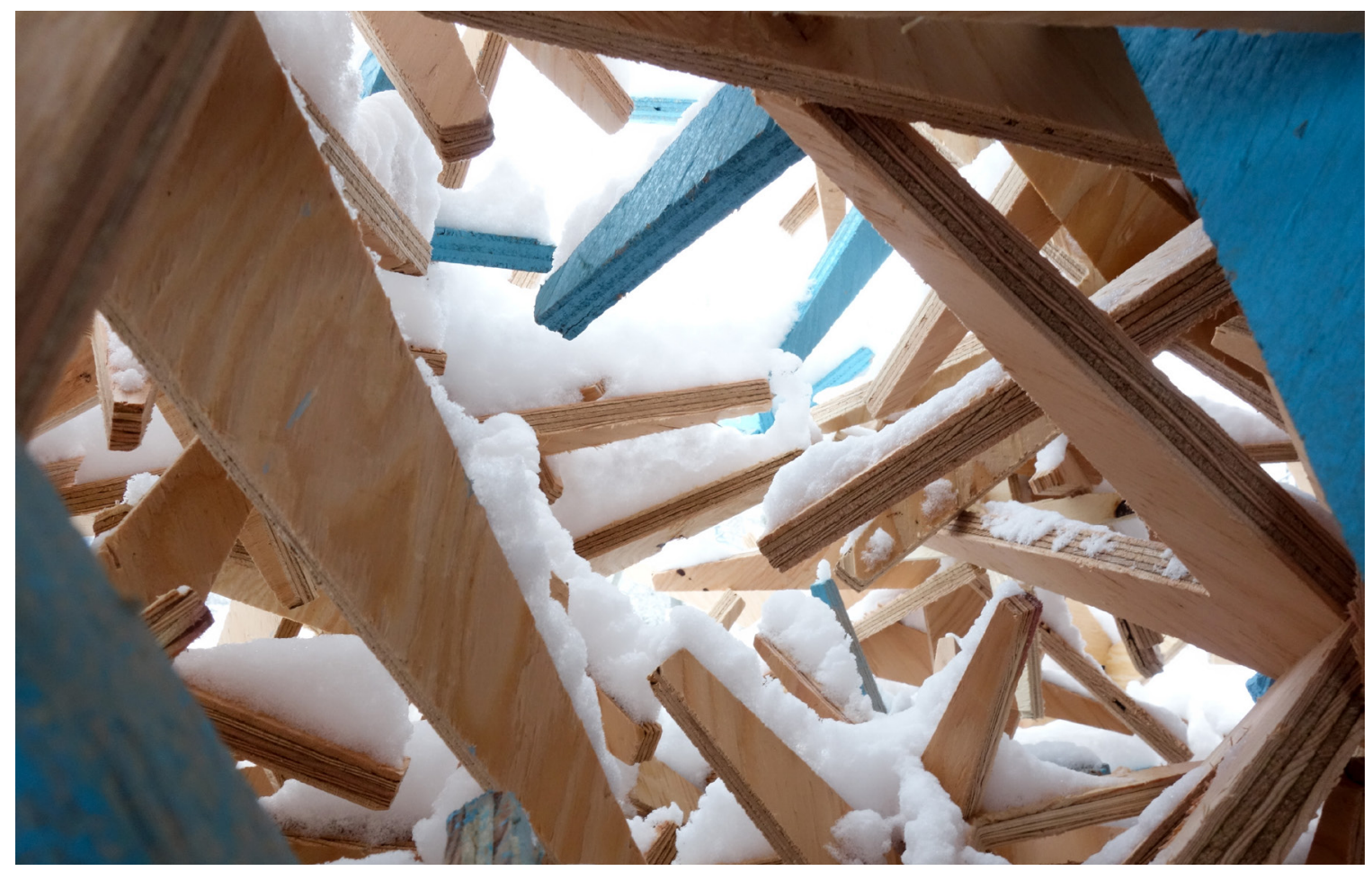

Figure 77. Close up view of snow accumulation from "inside"

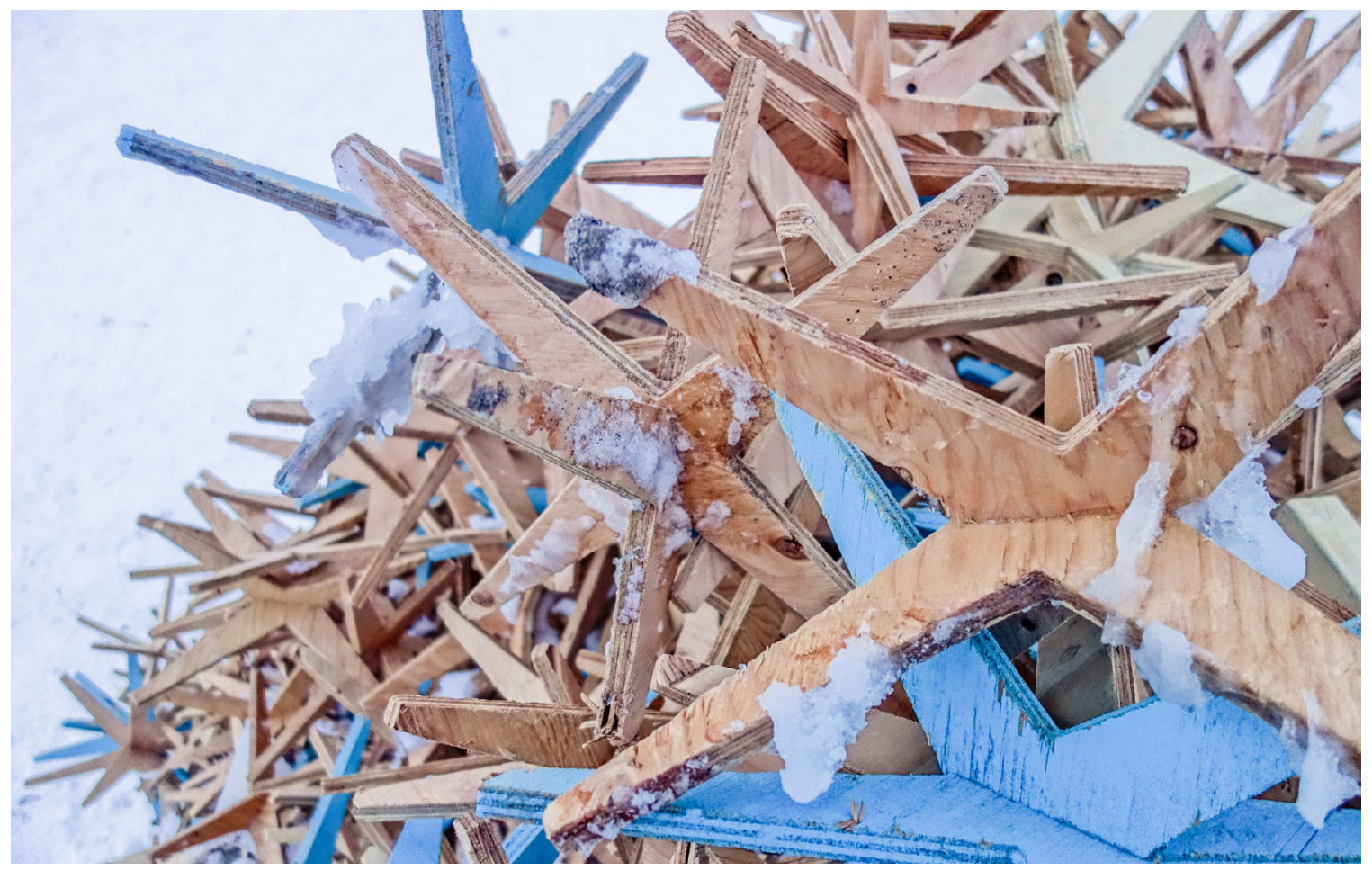

Figure 78. Ice buildup can bind particles together 


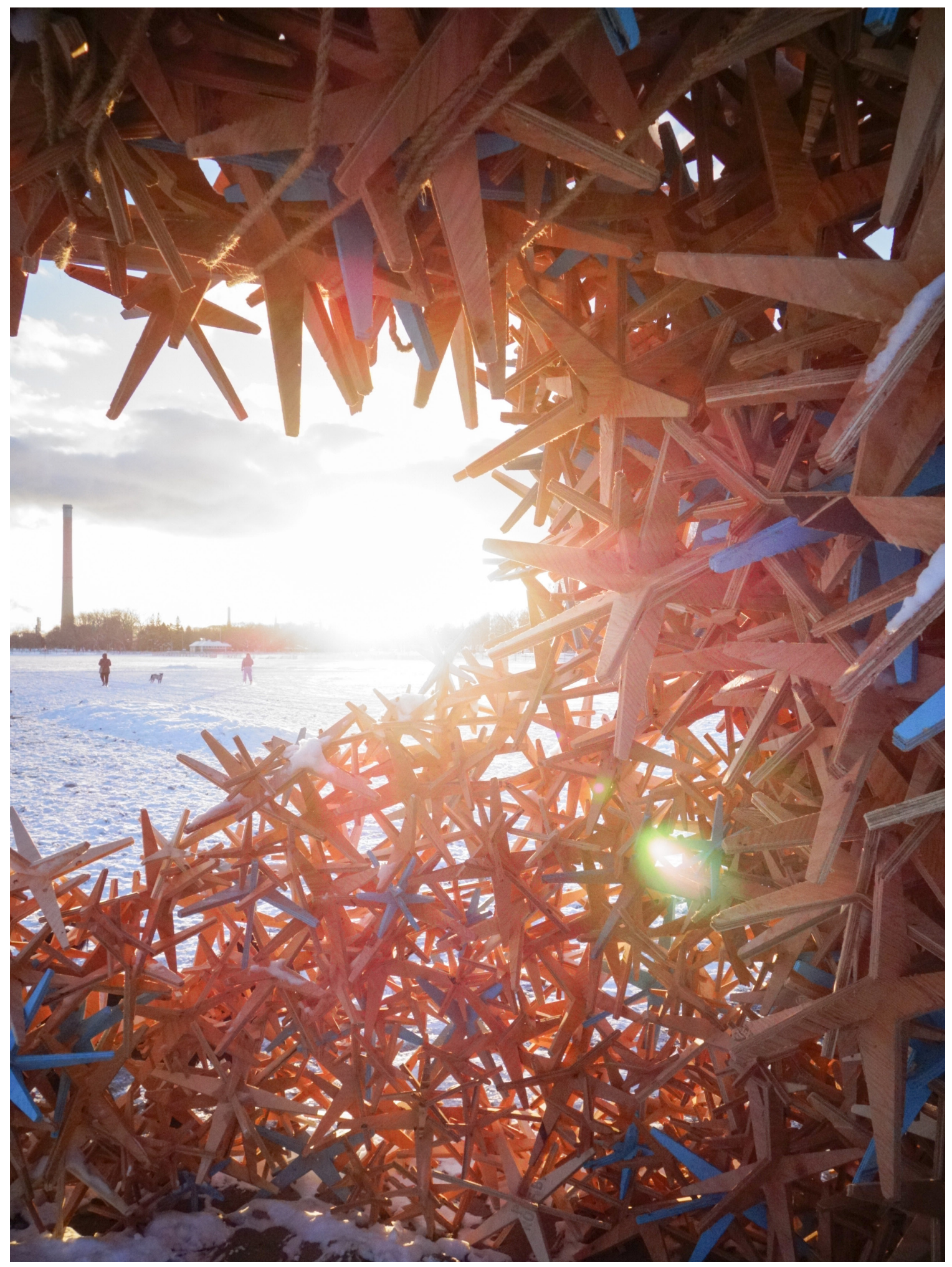

Figure 79. Flow filtering sunlight 


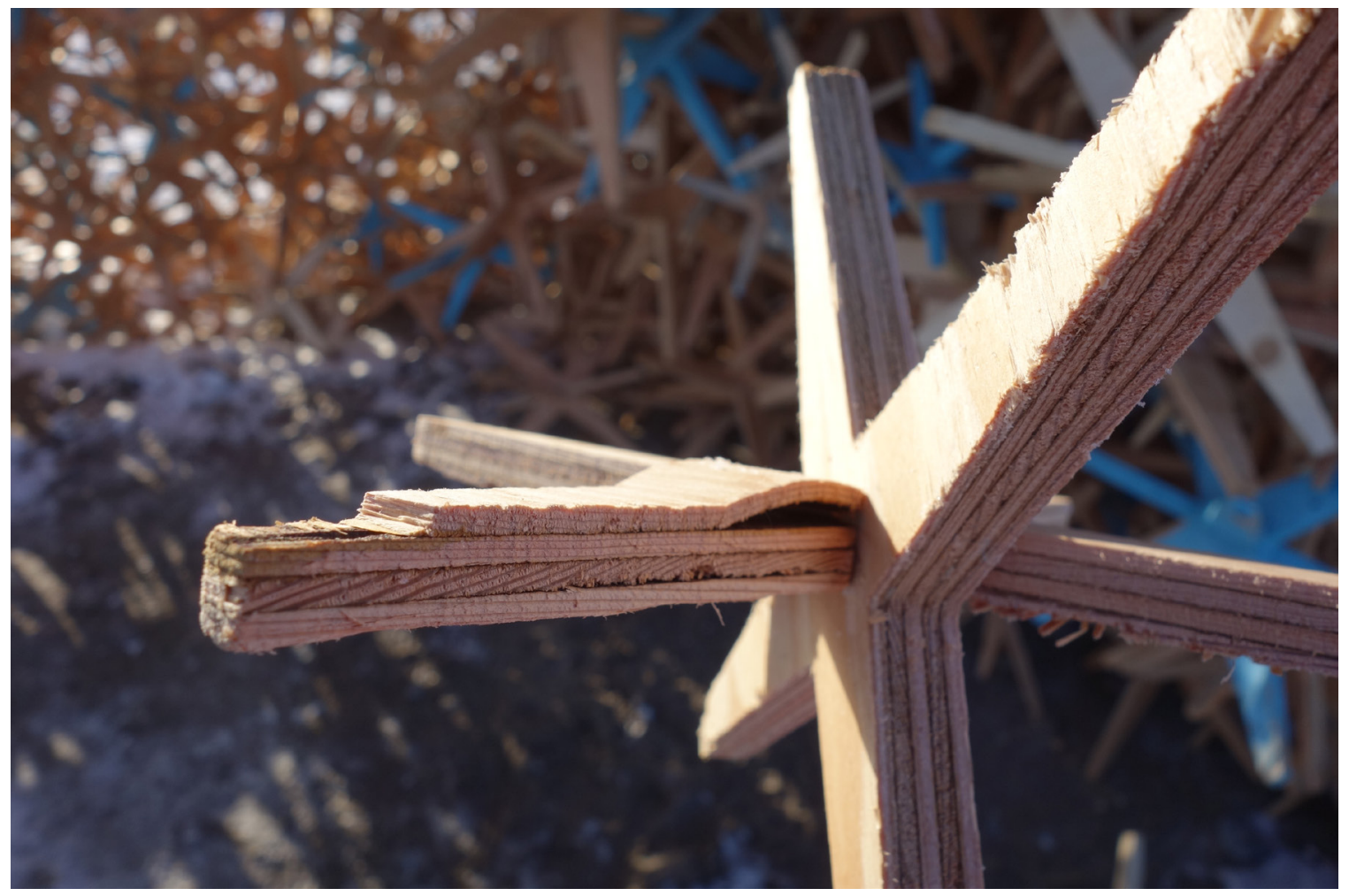

Figure 80. Component close up showing wear 

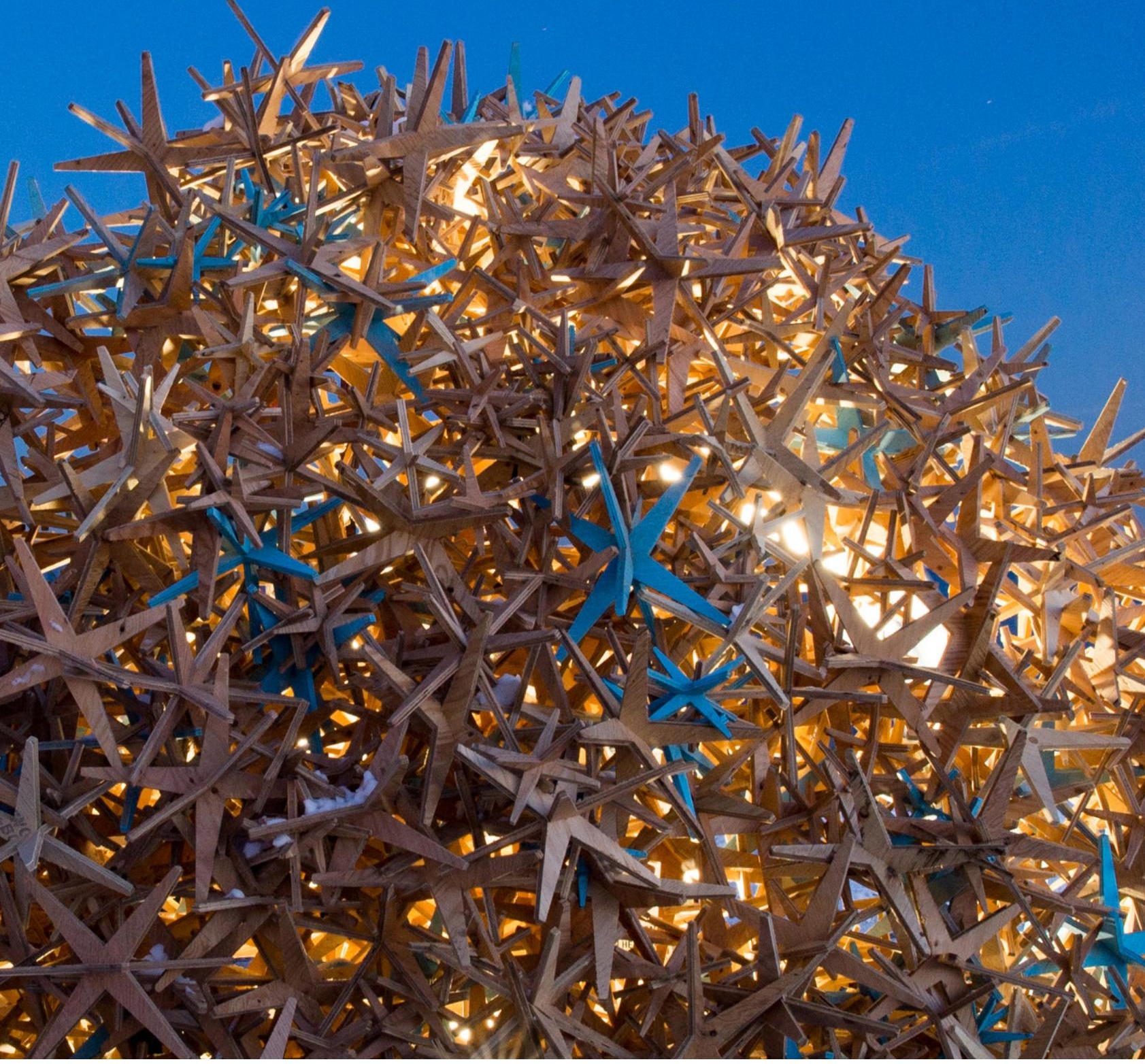

Figure 81. Lighting effects from interior in low light

122 Architecture at the Edge of Chaos 


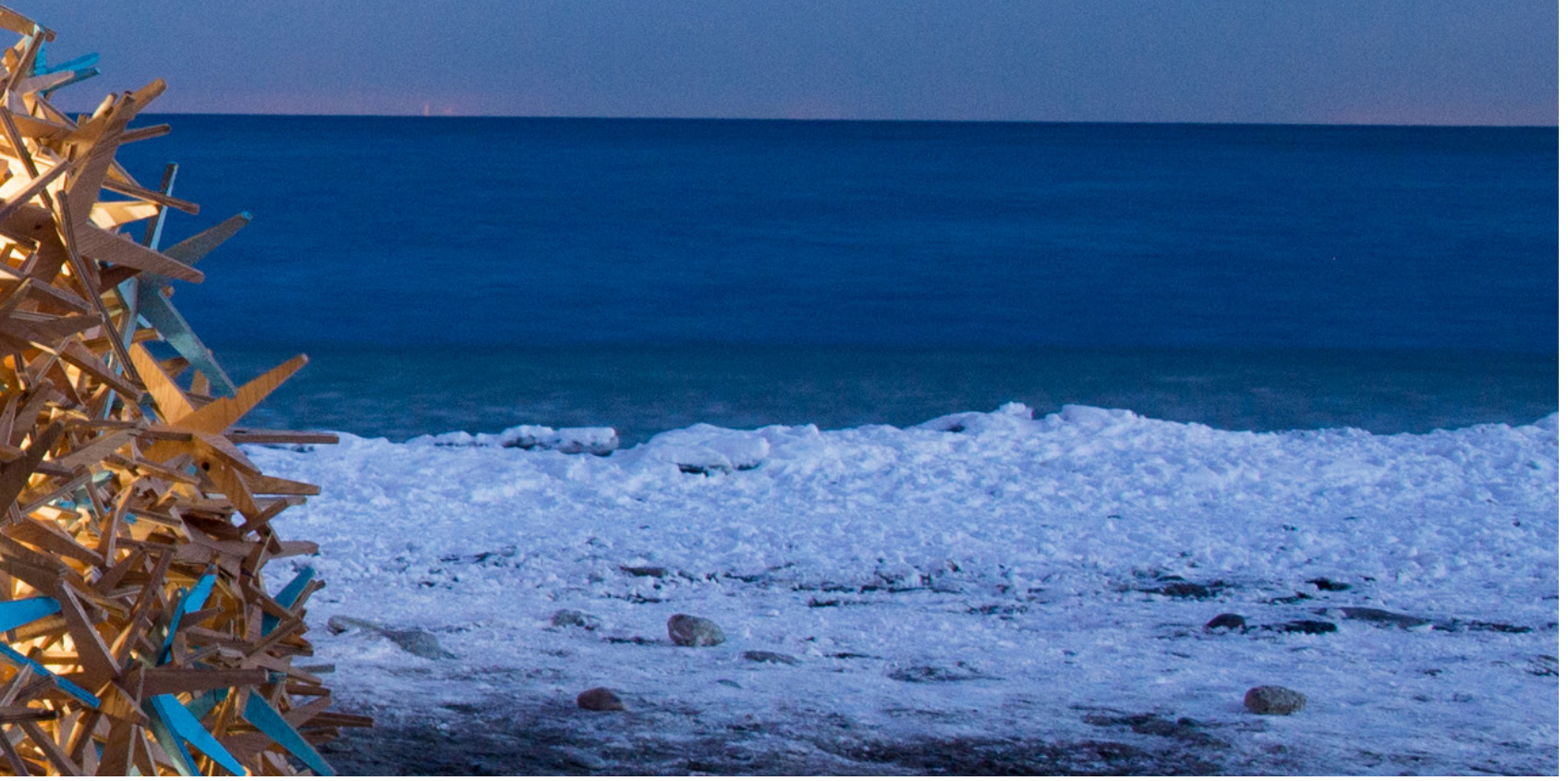




\section{Design Exploration 3: Seeds (Designing for Urban Agriculture - Allan Gardens)}

May-June 2016

\section{Objectives}

Upon the conclusion of the Winter Station event, there was an opportunity to demonstrate the system's cyclic potential. Due to the system's loose bonding, it was able to easily return to a free-flowing statedissolved to be reused and reconfigure again. The pieces from the beach were transported to Allan Gardens to be used in their community demonstration garden for urban agriculture. The new project required interventions that were movable and non-permanent to accommodate multiple community stakeholders which included children groups. This renewed life would confront issues of adaptation and durability as it faces a new setting, users, and function. More explicit functional objectives in this case provided clearer goals to evaluate its performance. The primary intention behind this exploration however, was to explicitly encourage and engage groups in building and constructing with the system, rather than an object of entertainment in the previous deployment (see Part 4).

\section{Concept}

The components from Flow were reinterpreted as "seeds" to fit the new design context and theme. There was a focus on temporary, disassembly, and reuse to maintain maximum flexibility. Witnessed from the Winter Station was the system's ability to quickly define space, provide visual interest and become host to natural systems such as snow. As such, these properties were furthered as screening (Figure 82) and possible trellis (Figure 83) for plant growth was proposed for Allan Gardens over the summer.

\section{Implementation}

The particles from the beach were packed into a 10 foot moving truck and delivered to the site (Figure 84). In terms of process, a dump truck typically designed for transporting loose material applicable and useful. Receiving the components from the beach, which had undergone heavy use, many of the components were found to be separated. This reiterates the focus required on the joint. Estimating quantity and the effective volume remains a challenge which better digital modeling can overcome. 


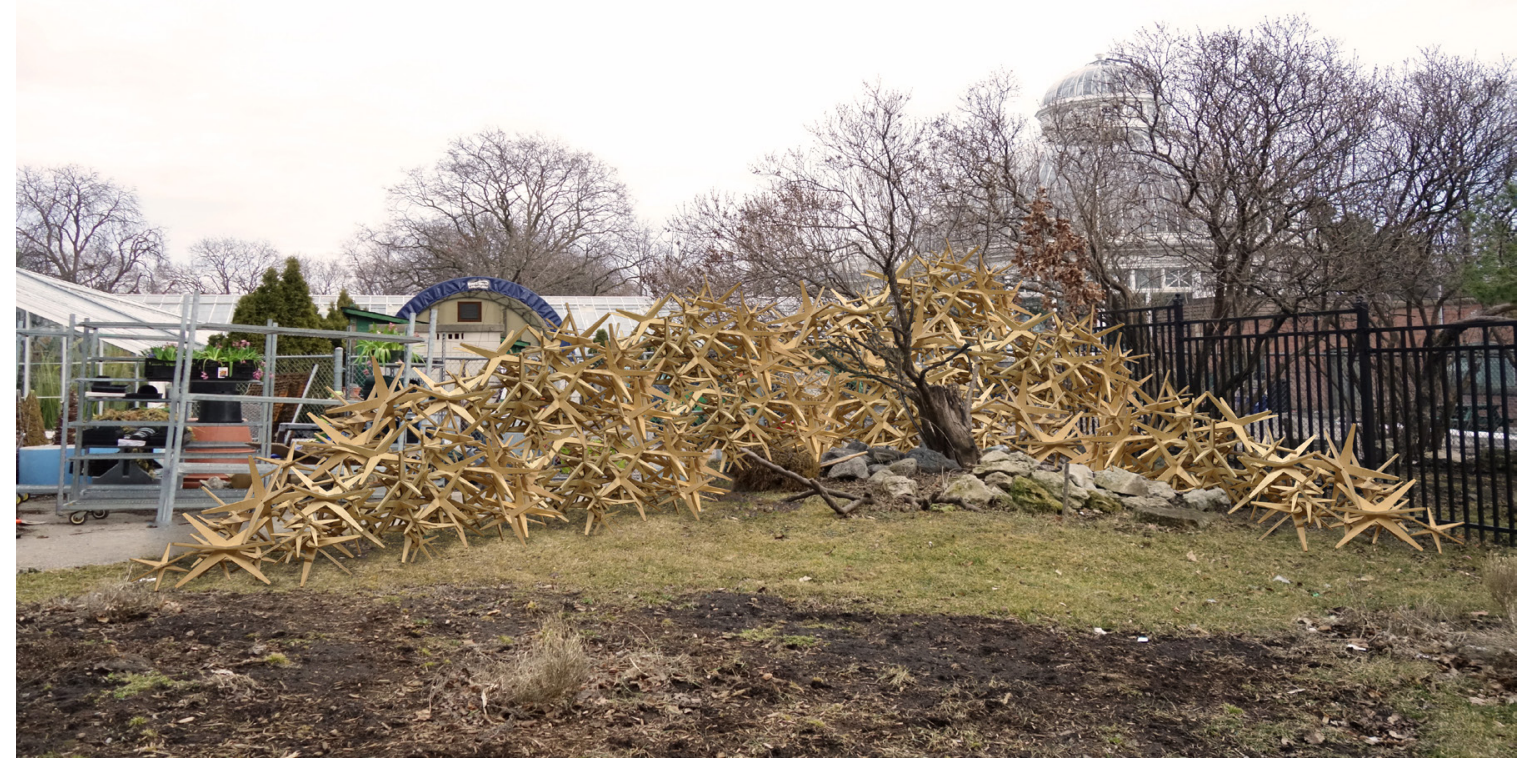

Figure 82 Visualization of screen proposed for Allan Gardens 


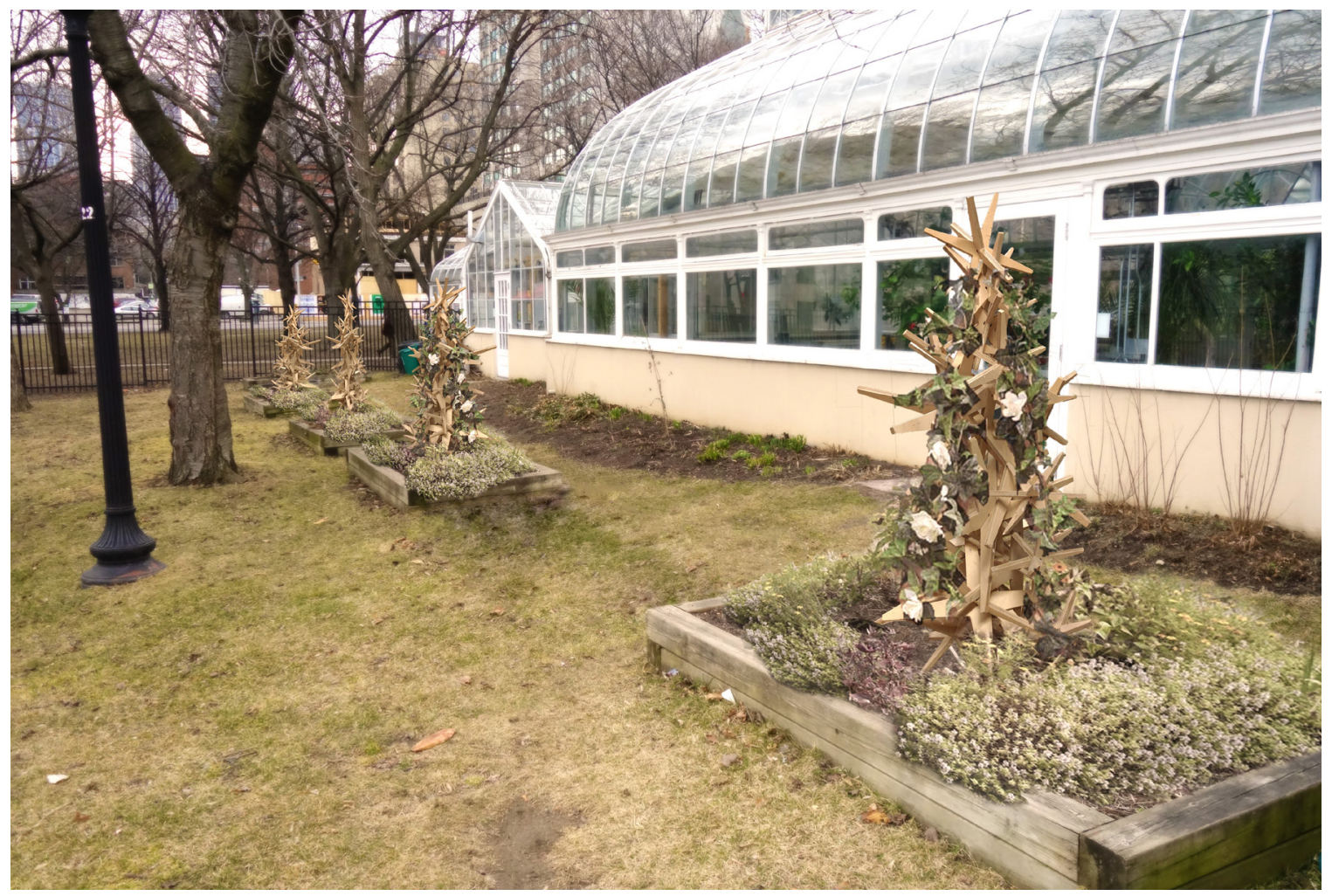

Figure 83. Visualization of pyramid trellises proposed for Allan Gardens 


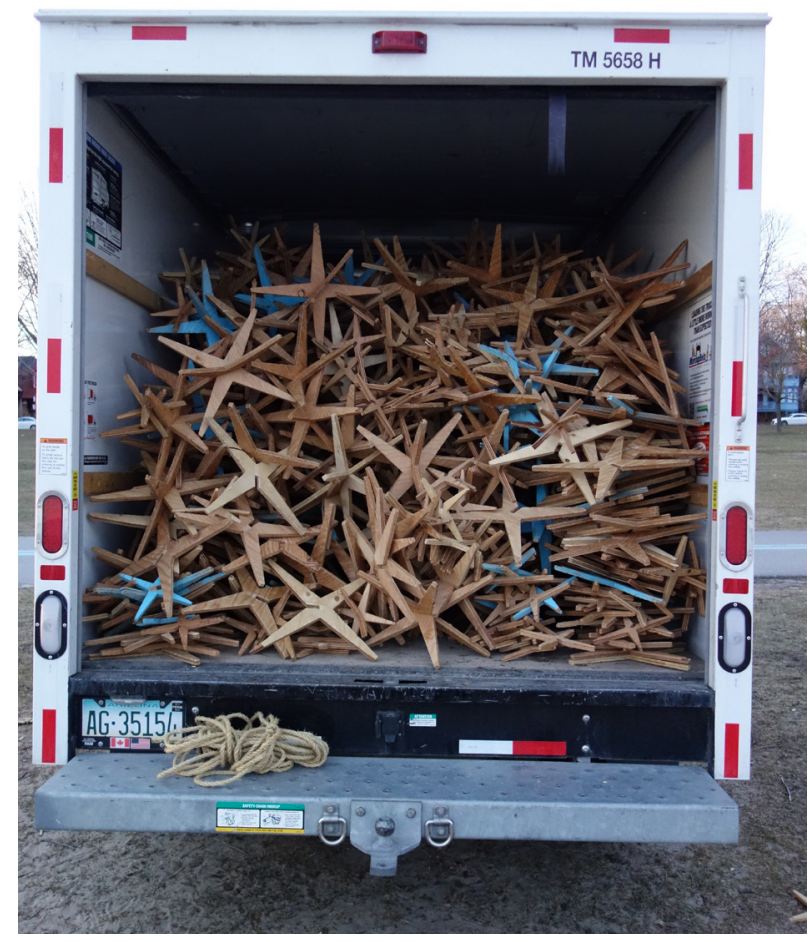

Figure 84. Transport of components

The main implementation of the seeds was as a screen/ separator required between the garden and its service area (Figure 85). The seeds were placed behind a light bamboo fence as a form of netting to discourage direct contact and reconfiguration once in place. With children actively using the site, a primary concern was the quality of the plywood finish which has gotten rougher and worn through use. This prompted the sanding of all the pieces, which in turn changes the properties of the aggregate (recall the discussion on surface friction).

Seeds also dotted the garden and served as a unifying design element. These newly fabricated units were finished with exterior paint in order to also prolong durability. The seeds complemented the overall design language of loose materials and recyclability which also employed gabion baskets filled with recyclables as seating and planters.

The new use and setting witnessed an engagement of animal wildlife not seen before at its previous deployment and context. Birds and squirrels have been seen navigating on and through the structure with its incorporation into the landscape (Figure 86). As for an armature for plant growth, the idea was never fully executed. A very limited test was conducted (Figure 87). It is imagined that the rougher plywood condition would greatly aid in the training of the plant and a cluster of seeds can guide and impact the direction of growth. 


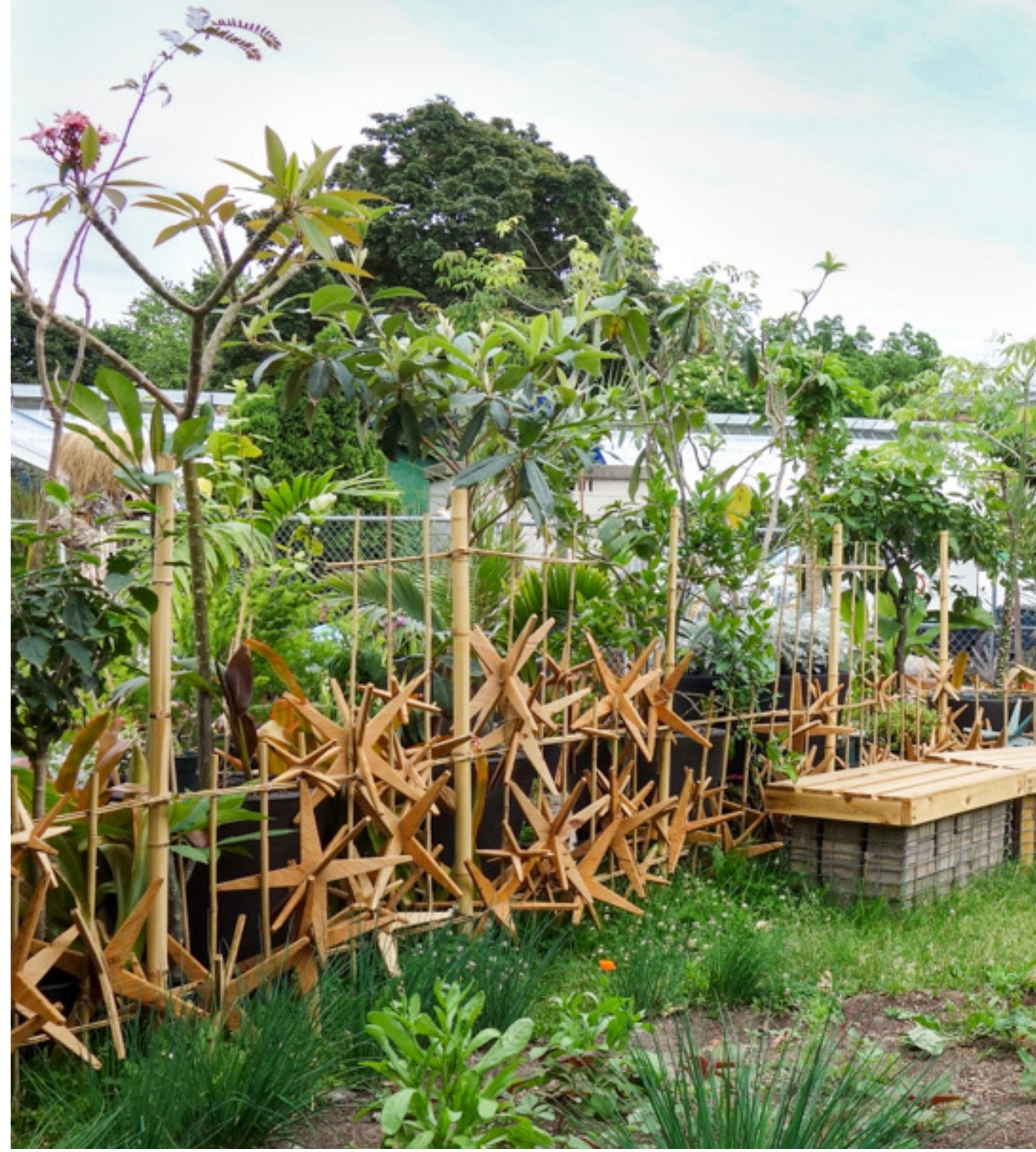

Figure 85. Overall constructed screen with seating 


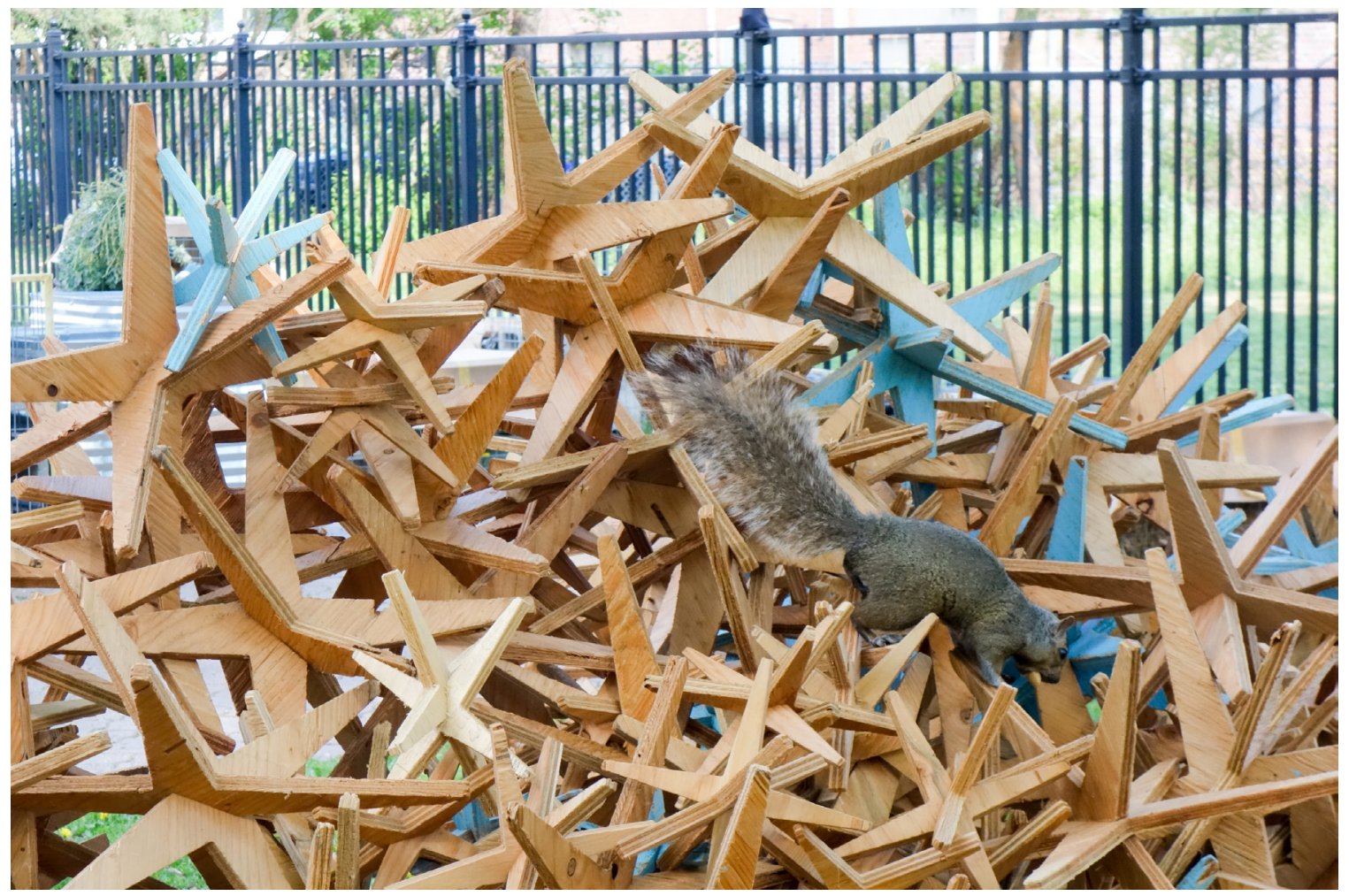

Figure 86. Wildlife engaging structure 


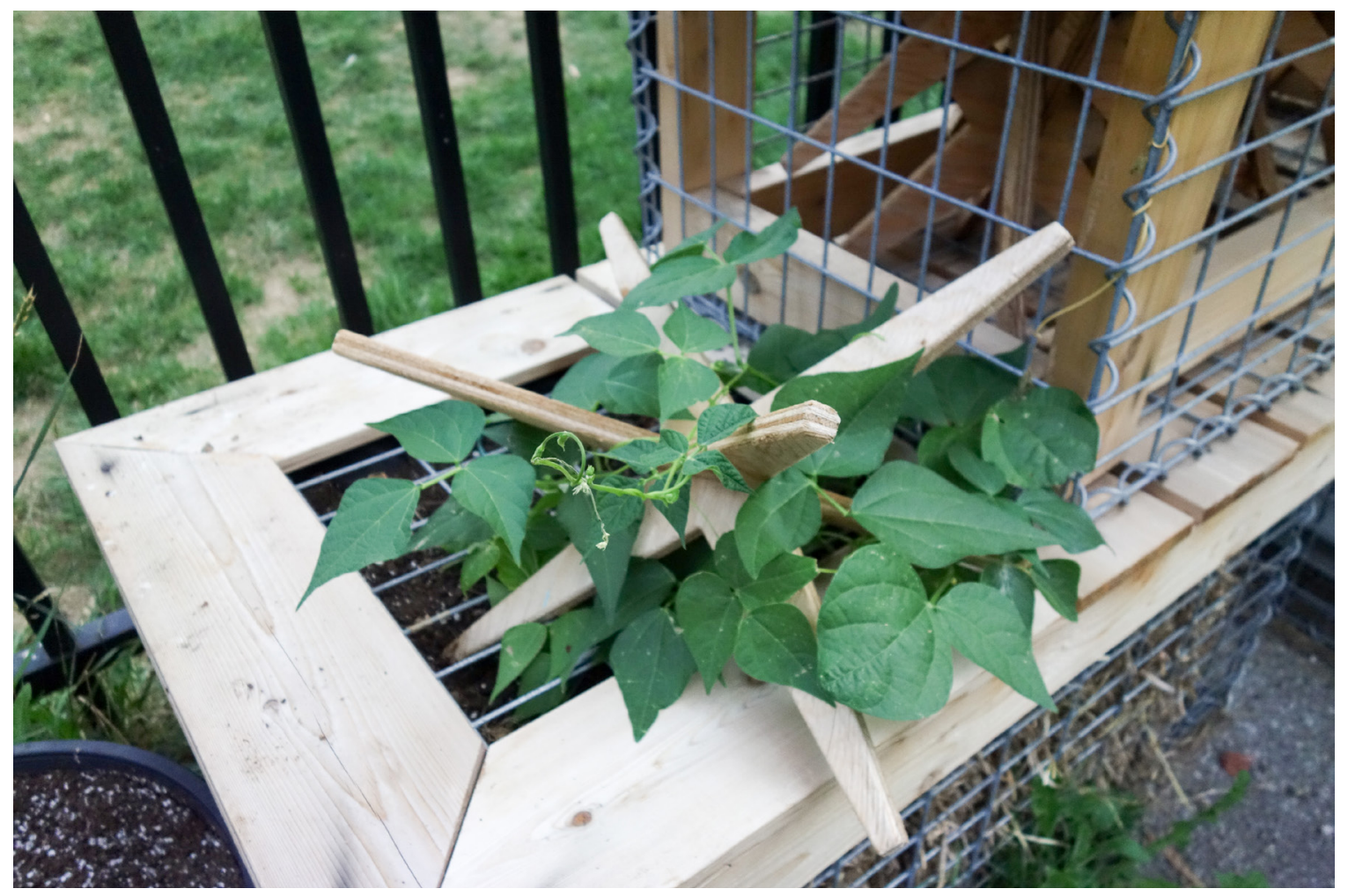

Figure 87. Seed as armature for plant growth 
The forms of nature change over time, constantly broken down and renewed. It is this condition that resonates with the granular system and provides for a more fluid conception of permanence. In fact, its material concept shares many intrinsic principles of sustainable construction practices. It is possible that this implementation is only but another iteration of many for this seemingly simple pile of wood. Its modular elements show a large amount of variation in design and enables many possible futures. An architectural material conceived as such, as a "soft" and "cyclic"-fluid and able to adapt and transform-begins to accept the contingent forces of the world it has resisted and initiate alternative forms of thinking and practice in architecture. The intelligence, agency, and performance afforded by material challenges many notions in architecture, but in doing so opens up new relationships and greater possibilities.

\section{Case Study: Fab Tree Hab - Terreform ONE / Mitchell Joachim}

The desire to continue the potential idea of integration with the natural environment, in this case the possibility of serving as an armature for plant growth, is reminiscent of the Fab Tree Hab home concept by Mitchell Joachim of Terreform ONE, where a living structure is grafted onto reusable CNC plywood scaffolds (Figure 88) (Joachim, n.d.). It employs a technique called pleaching where tree branches are woven together resulting in a strong structural form.

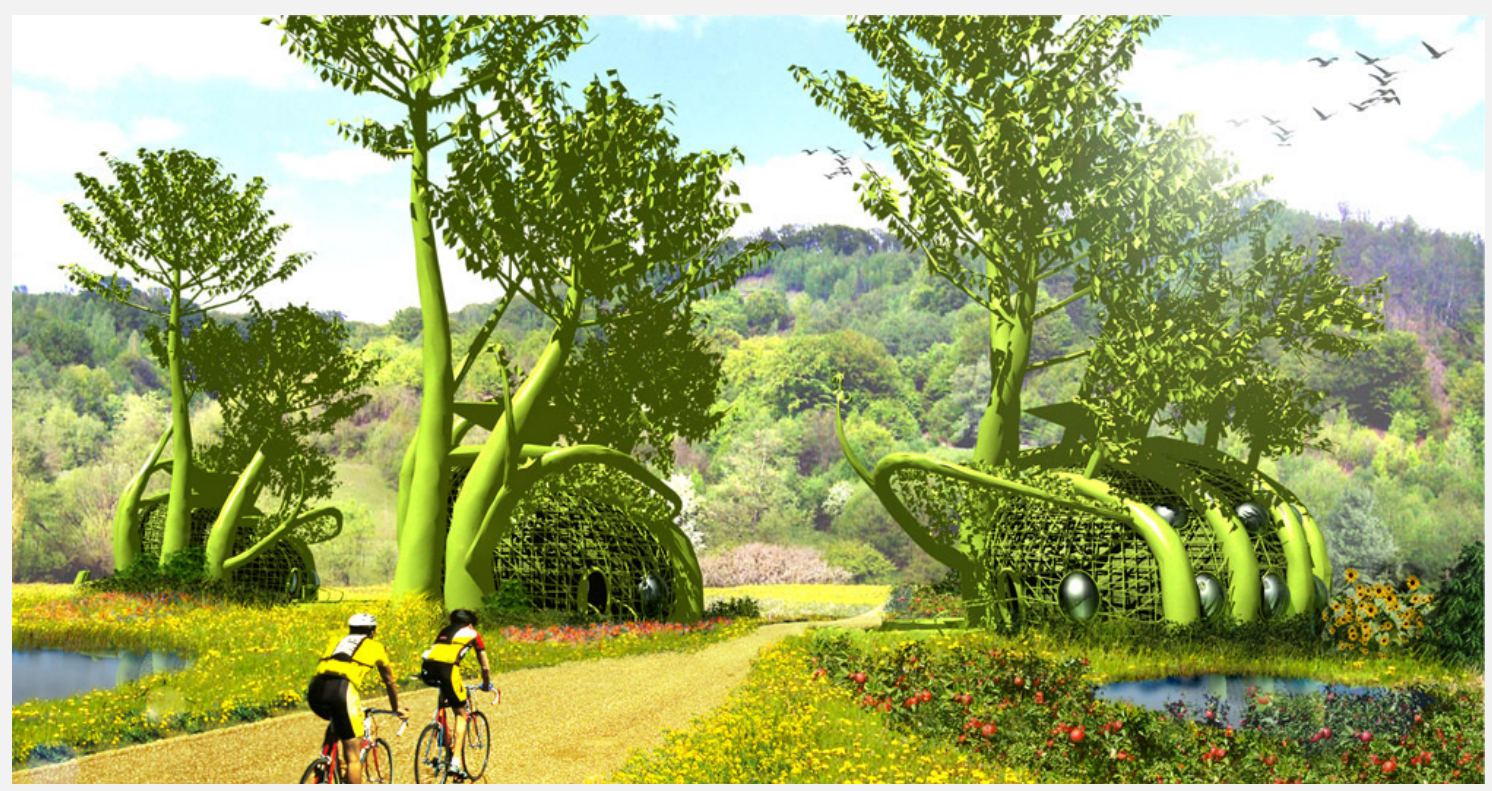

Figure 88. Fab Tree Hab Concept. From Terrefrom ONE 
The use of the Seeds could easily compliment this concept, rethinking its idea for the scaffolds as an integrative component that can be programmed, in turn permitting the "design" of the plant material. Granular materials, as has been seen, consistently recalls/evokes its connection with natural systems, working with nature rather than resisting it.

(Refer to Appendix D for additional images.)

\section{Materials and Methods}

Designed granular material systems challenges current modes of architectural design practice and introduces new concepts, methods and tools. The unique aggregate material behaviour and endless formations from one stable state to the other is seen to challenge the architect's rigid precision and limitations of preconceived design ambitions. Architects conventionally control the exact global form of a structure. However, here, the geometry of the local element, the granule, "is defined not to achieve precise geometry but rather to calibrate a certain material behaviour with a scope of possible formations" (Dierichs \& Menges, 2016). The shift promotes design methods grounded in process and evolving formation rather than a finite end product, where the loose granular systems ability to calibrate the ultimate state is used to configure and re-configure form. The process of designing and building become inseparable and operate simultaneously. 


\section{PART 4: IMMATERIAL ARCHITECTURES}

"(Social) space is a (social) product [...] the space thus produced also serves as a tool of thought and of action [...] in addition to being a means of production it is also a means of control, and hence of domination, of power."

— Henri Lefebvre

The Production of Space (1991) 


\section{The Creative User}

There has been very little explicit discussion in this area of research on the implications of granular material in a wider architectural and social context—on architecture's immaterial structures. Most have been focussed on the material and performance. Jeremy Till and Tatjana Schneider cautions on the danger, particularly with architects within these types of open building concepts projects, to become obsessed with the technologies and it eventually becoming the end in itself rather than the means (2005, p. 294).

What the Design Explorations revealed, which was initiated with the physical realization of Flow, was the enormous social potential within the material. In working with such system and reflecting on the role of the designer, one may free themselves from the responsibility of the exact, final, physical form, and instead focus on designing general performance and ambiances—catalysts for possibilities. This shift, admits the user into the design process — an agent of great unpredictability most certainly, but one that simultaneously possesses the exact information needed to address the challenges of adaptability. Tuned and programed by a new type of architect, the designed granular material is seen to have its own agency, able to self-organize to its stable state and as such, permits the agency of the user and forces of uncertainty. The accessibility and response to people observed is very high due to its low barriers for involvement. A greater degree of expression, desires, and change can be achieved and hope that people can start to craft their own environments.

\section{Flow}

Flow attracted an intense level of interaction from the public. While the competition brief mainly referenced the dynamism of the weather and site, which focused the efforts towards the physical research, the greatest influence of change would come from public participation-something that would be far beyond our control. The structure began as an arched form, but would radically change throughout the 39 days in ways that far exceeded anything anticipated. Loose granular system, it had been observed prior from the material tests, has an innate ability to engage people's creativity and play. While this had been recognized and even quietly desired, first news of people's engagement still came as a big shock. It was never originally made explicit that people were encouraged to reconfigure it, but that only the system could accommodate change. It was unsure whether visitors would embrace interaction or be intimidated by the preciousness usually placed on art pieces. 


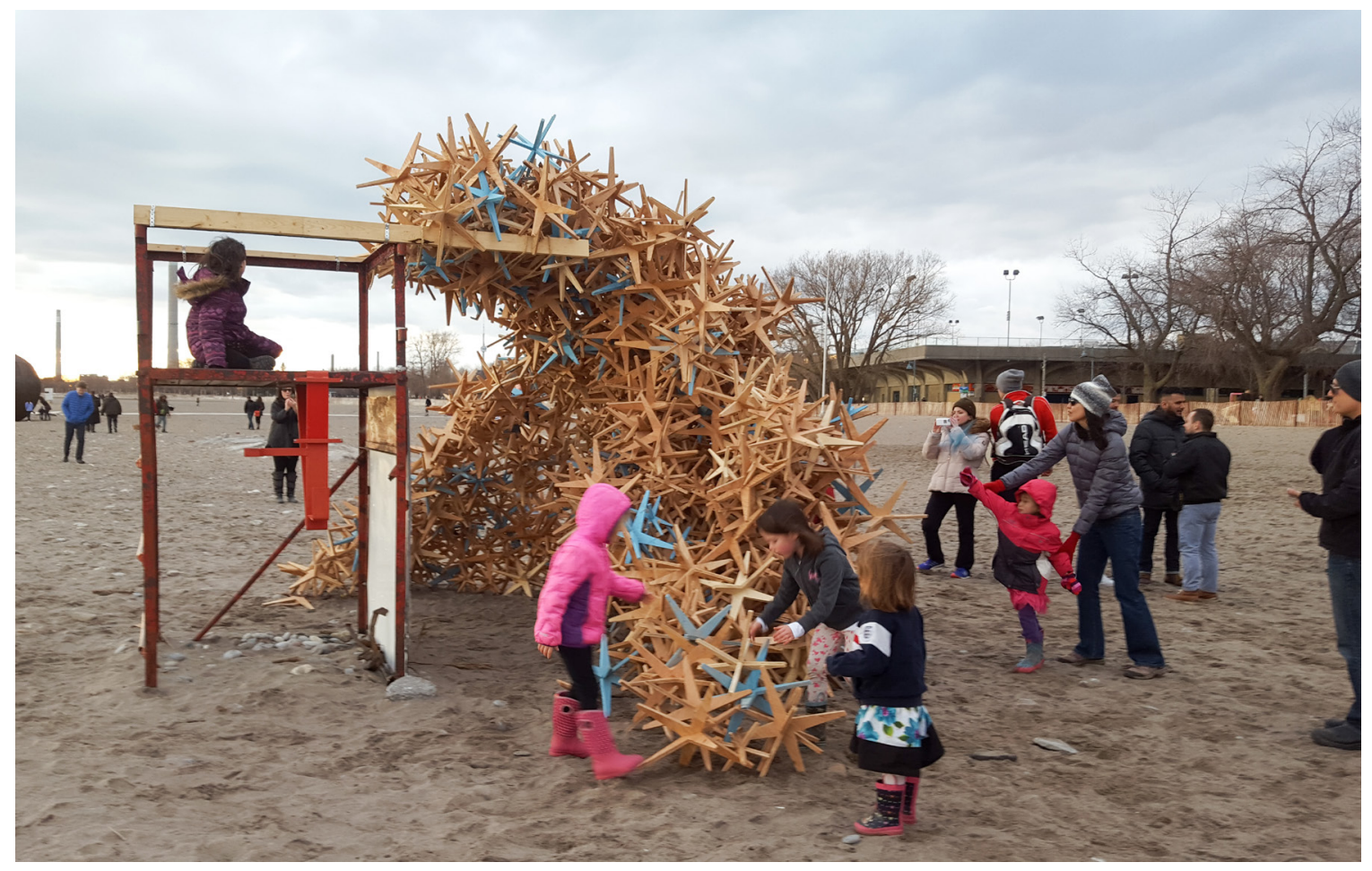

Figure 89. Visitors discovered that the form can be modified

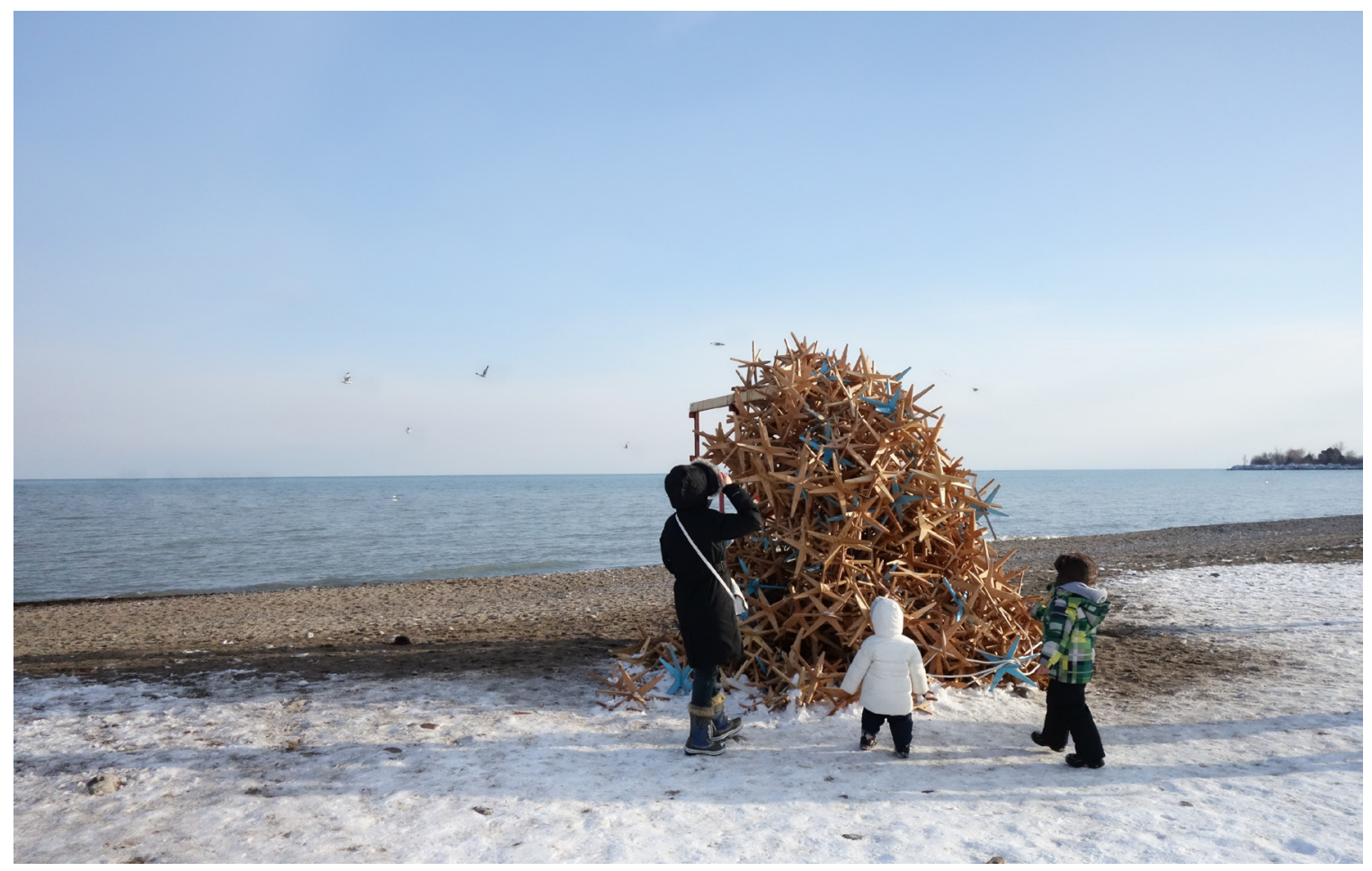

Figure 90. Some visitors unsure whether to engage 
It remained in its initial state for a week before a particularly warm weekend drove many to the beach which was when it was discovered that the structure could be 'hacked' and modified (Figure 89). People were generally impressed by the discovery of how the structure was held together, without fasteners, which in turn usually leads to an attempt to remove a component. Many at first were apprehensive and unsure whether it was permitted, to the degree that a debate was witnessed regarding whether the structure should be touched (Figure 90). Here we experience what Hill describes in relating architecture to traditional art such as gallery-based painting or sculpture. He states that architects have desired the elevation of architecture to the status of art to affirm status. The "user" is thus equated to "viewer", expected only to observe and contemplate passively (2003, p. 22). Preciousness is usually ascribed to art pieces and the unruly influence of user is resisted.

As time progressed and as more people engaged with it, people became more comfortable with the premise. The control of form had been ceded to the public (Figure 90). People enjoyed throwing the components and watching them interlock or seeing how high it can stack. There was also something very satisfying to people seeing it collapse. The installation would continue to evolve and change in unpredictable ways and not just in terms of form, but the entire system including the people and the environment. Visitors became more aware, more comfortable, stars would be taken, pieces would deteriorate with successive throws, and it was never certain whether the next particle added to the mass would bring it crashing down. Through social media (Instagram) a public record of the structure's evolution was captured (Figure 91). Form here is the direct expression of the human system-a type of crowdsourced design.

It would support a form of interaction beyond what is typically experienced in art and in particular architecture. It does not reset itself after the next person, but an active record is maintained. The material contains a memory where every contributor would leave their mark and it remains a part of the story and life of the installation. Each successive move builds upon the previous and in turn informs the future. D'Arcy Thompson views the form of any physical object in terms of its shaping forces and the pattern of its historical development (Moe, 2013, p. 290). Mark Wigley makes a similar observation from a social perspective on the impact changes have on New Babylon:

Every transformation of the space, no matter how minor, is understood as a direct intervention in social life that sets off a 'chain reaction' of responses. New forms of behavior evolve interpretations and reactions emerge, only to be challenged by the next spatial move, and so on. (1998, p. 14) 


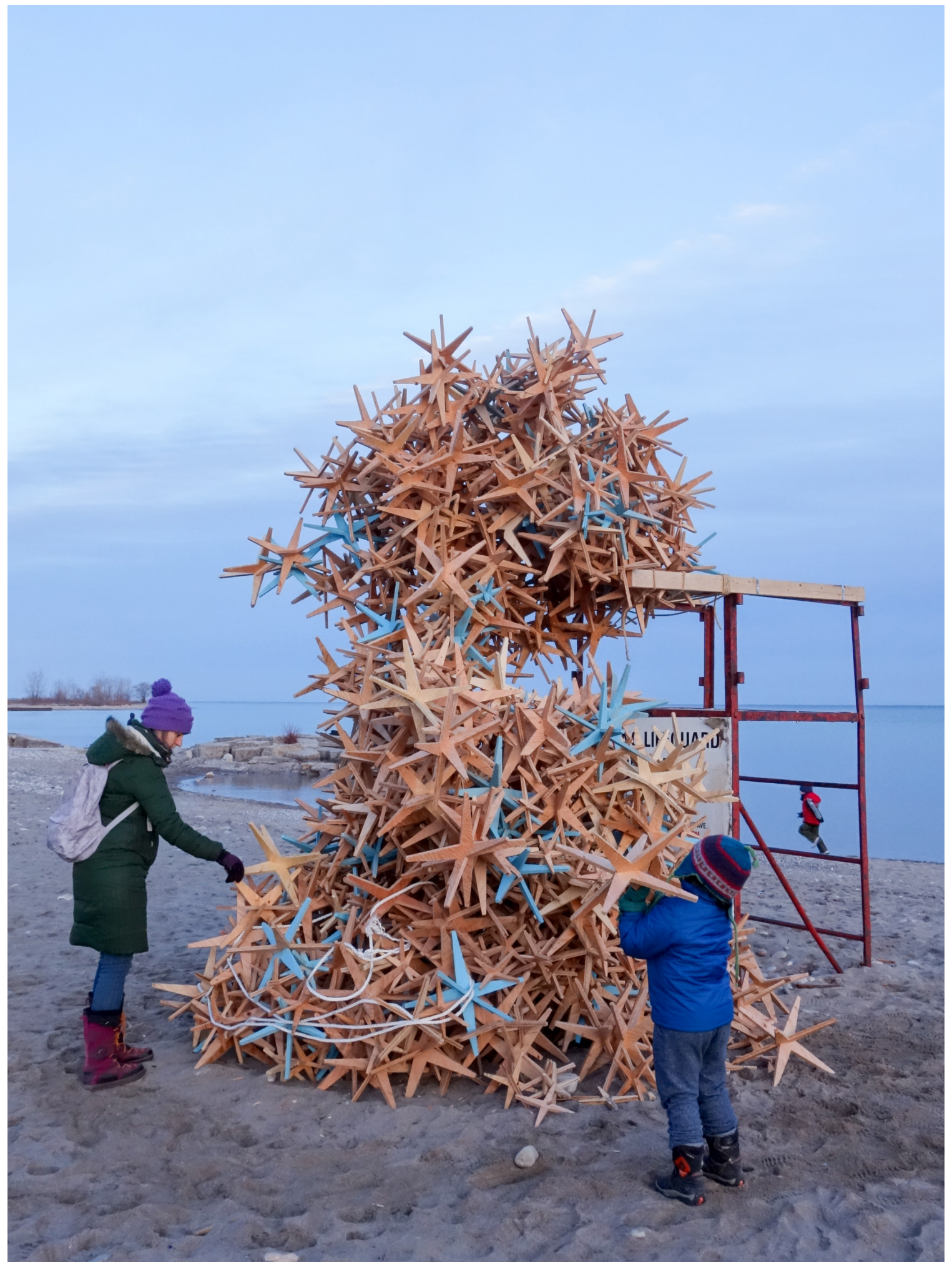

Figure 91. The form would continue to evolve 


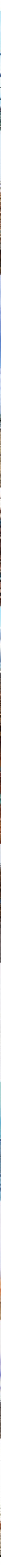

140 Architecture at the Edge of Chaos 


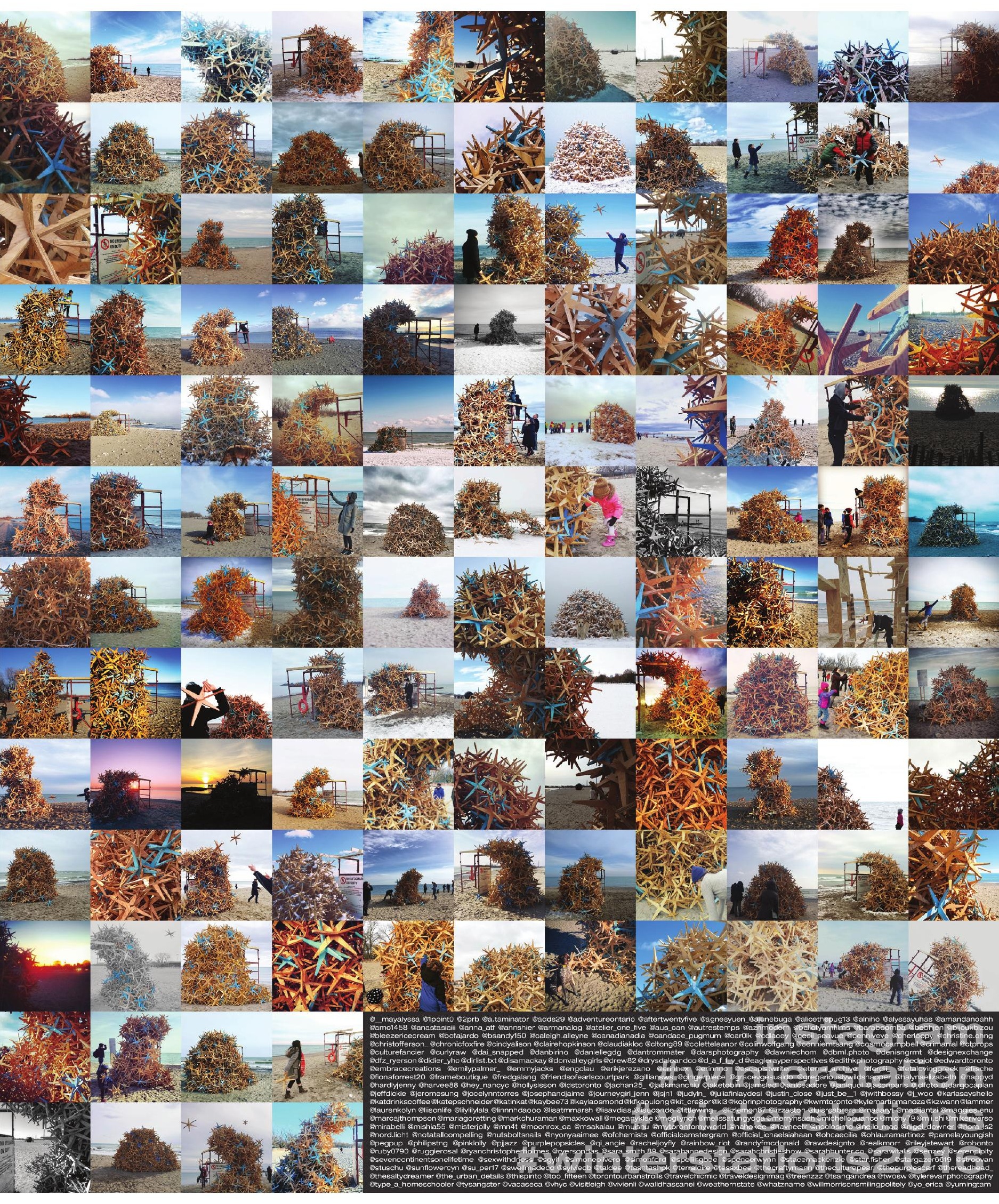


In general it remained in the form of a pile most of the time, though there were few instances where people would try to work with it like a building block to craft a form rather than merely tossing it to see it stick (Figure 93 and Figure 94). It was explored, it seemed, more as an interactive "target board" or toy than a type of construction block (Figure 95). Only towards the end of the event, two days before it was taken down, did a cave-like form start to emerge (Figure 96). Whether it was constructed on purpose or by chance is unknown. Manolopoulou offers this relevant point:

"the architect should dare to observe and thereby discover. Acknowledging that architecture is co-produced by both design and chance requires us to learn how to better observe in order to critically decide where and when to design and where and when to stop. When the chance situation is given as a positive "found environment', then non-design, or designing less, are valuable design tactics. Recognizing the beauty of indifference found in buildings and places that are made without particular design intention is important: architecture must have some of this beauty of indifference in order to be less dominating, otherwise it risks being too precious to inhabit." (2013, p. 215)

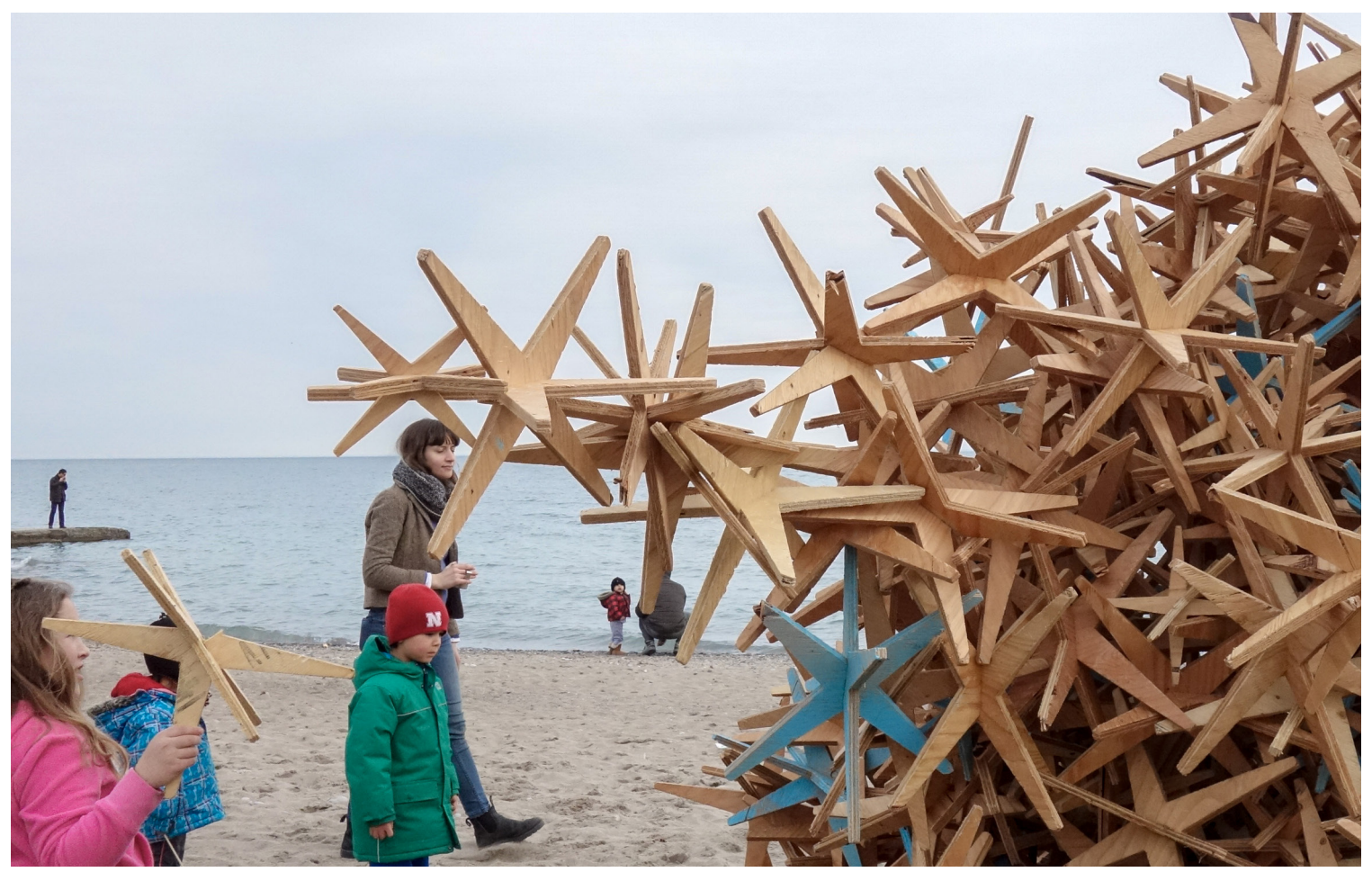

Figure 93. Form can be carefully sculpted 


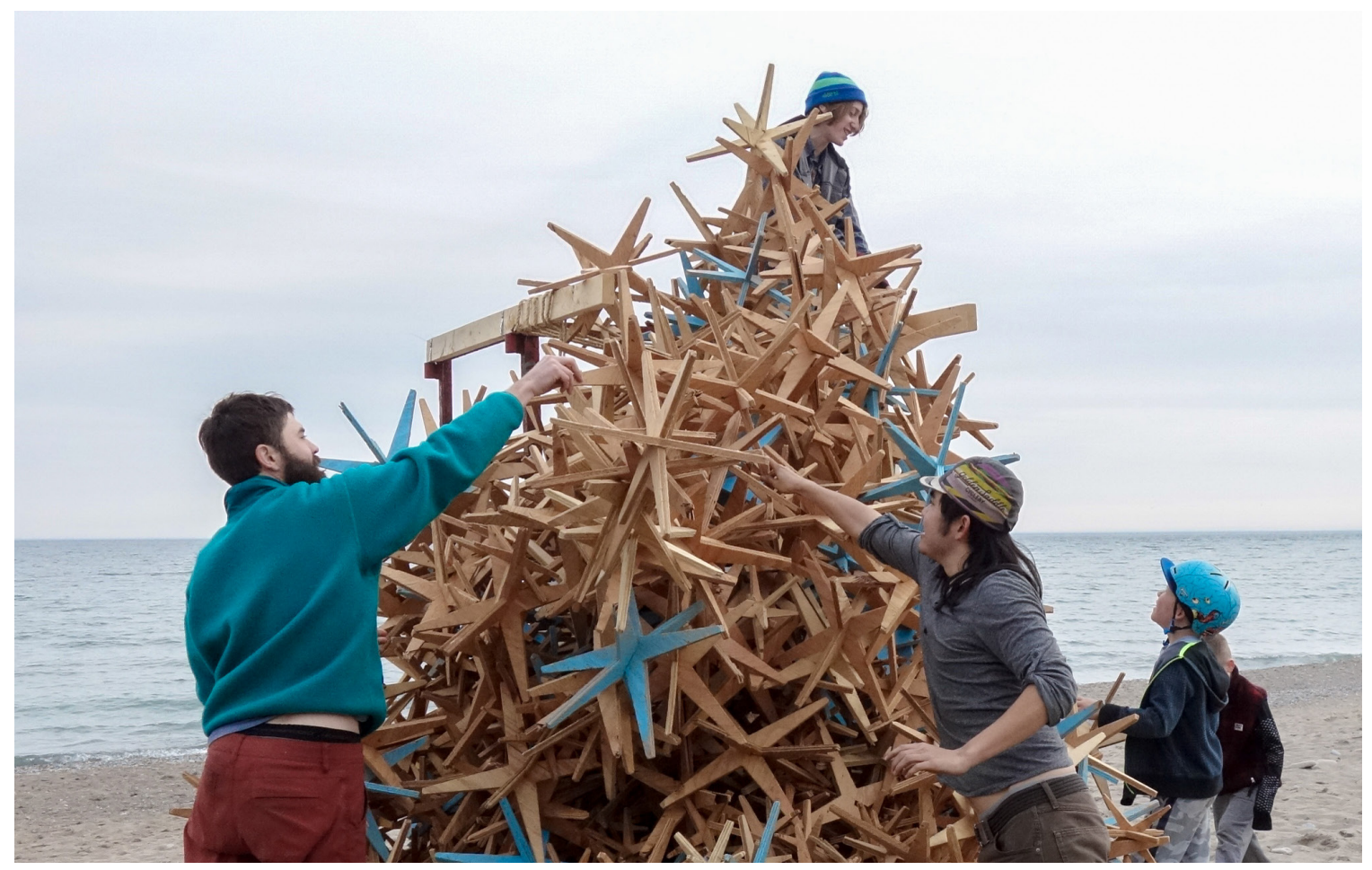

Figure 94. Through participation, the form remained mostly as a pile

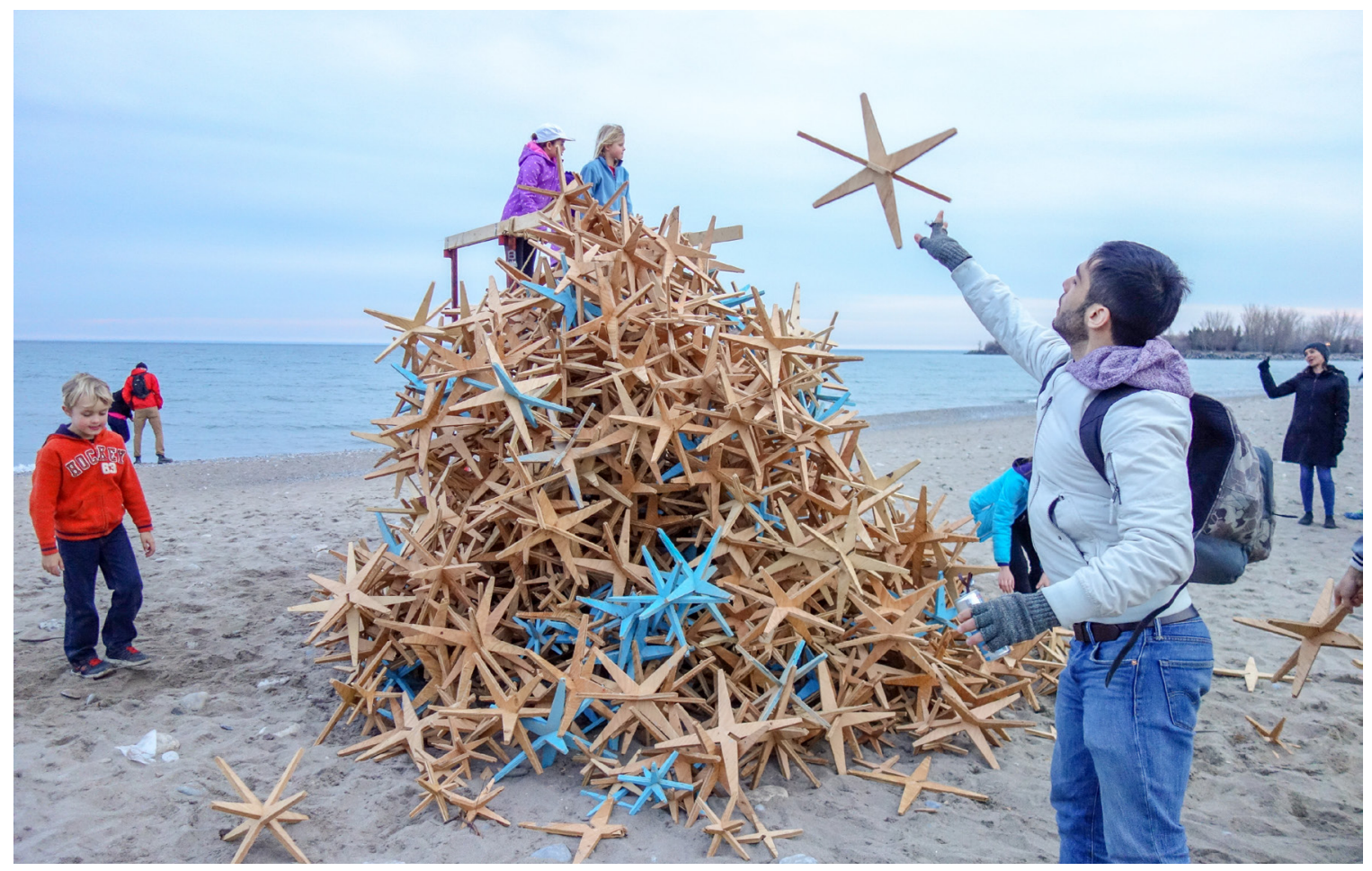

Figure 95. Visitors typically like tossing the components 


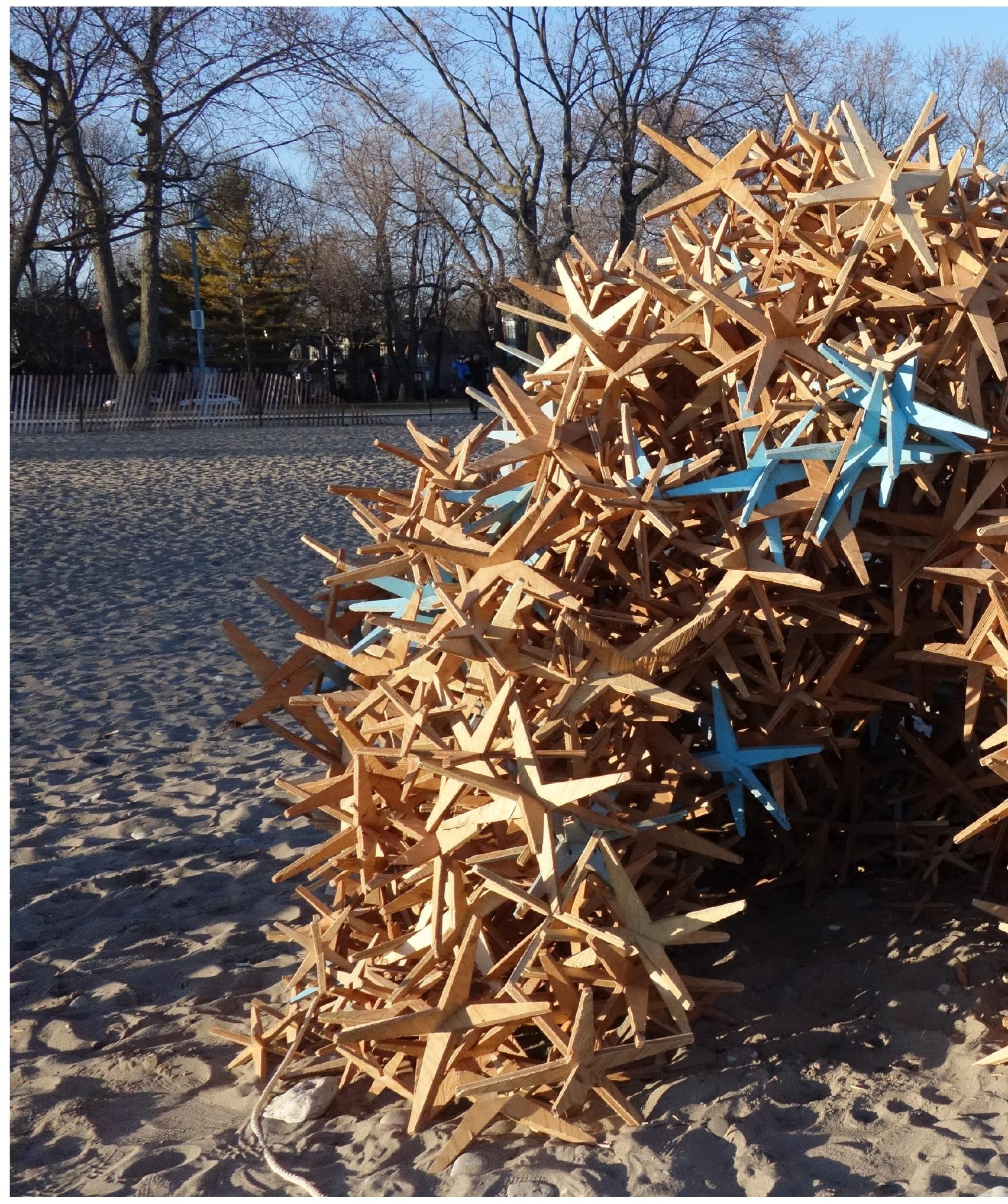

Figure 96. State on final day

144 Architecture at the Edge of Chaos 


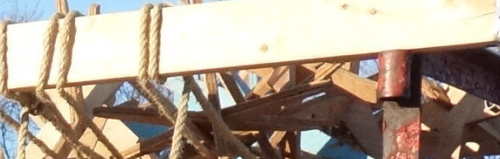
$-1(x-2)=1$

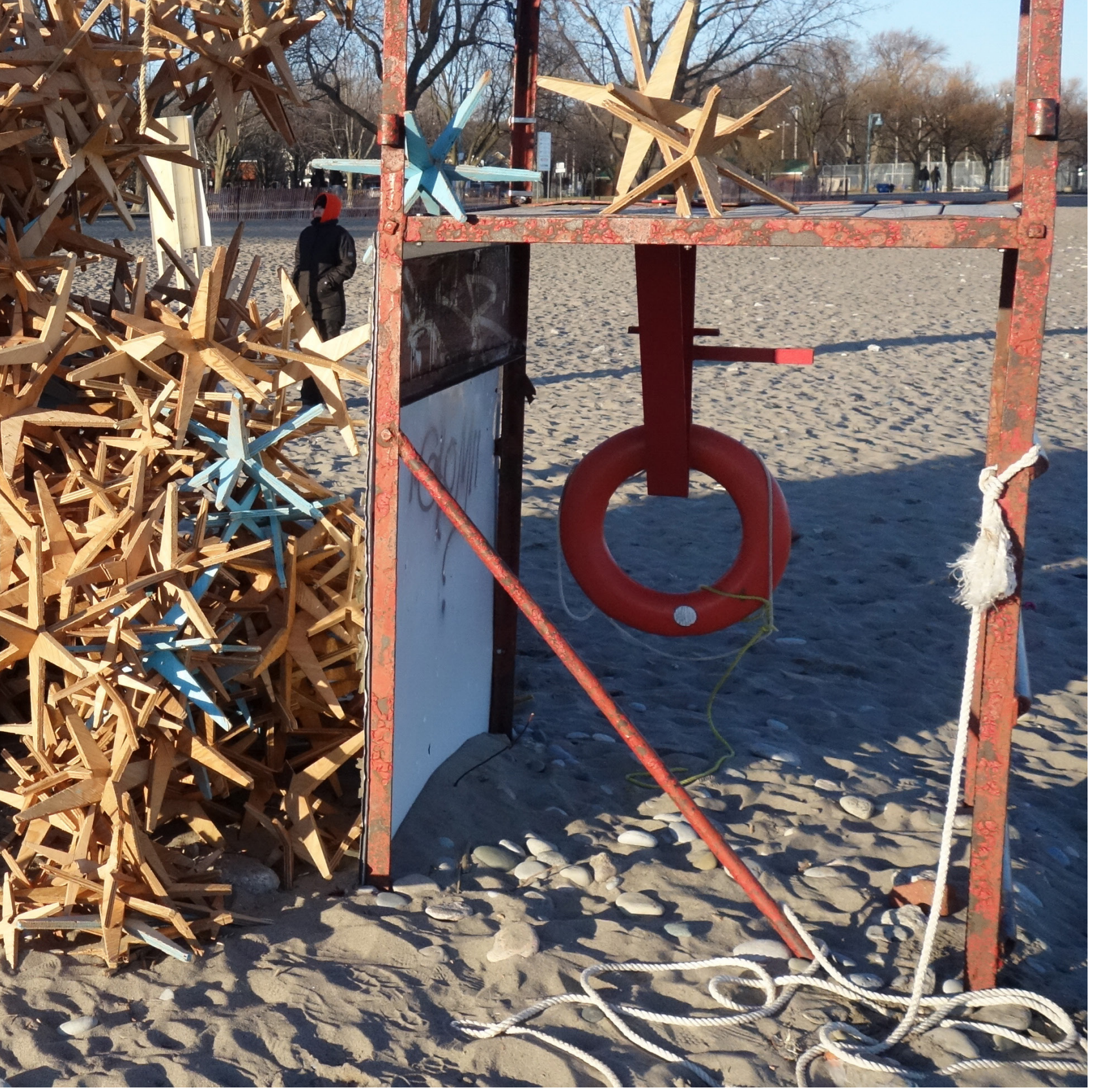




\section{Case Study: Bloom - Alisa Andrasek \& Jose Sanchez, 2012}

In a project similar in nature, created for the 2012 London Olympics, 60,000 identical plastic units, were created for a "crowd sourced garden." BLOOM, designed and developed by Alisa Andrasek and Jose Sanchez from The Bartlett School of Architecture, is an urban toy/ game that seeks to engage people in social play and design (Figure 97). "BLOOM considers a mode of assembly, disassembly and re-usability that challenges the notions of traditional construction" (The Bartlett School of Architecture, UCL, n.d.).

Each plastic "cell" contains 3 discrete slots for connections, but it can be combined in a number of ways that end up producing forms that are impossible to predict. People are able to add the pieces to an initial structure to alter its form or start creating new ground sequences that can be used as urban furniture or simply unpredictable formations. The collective act of coming together and building something becomes a shared memory for each person. None of the pieces can do anything individually, it is only with many pieces, and people coming together that something meaningful emerges. As such, the game is open-ended.

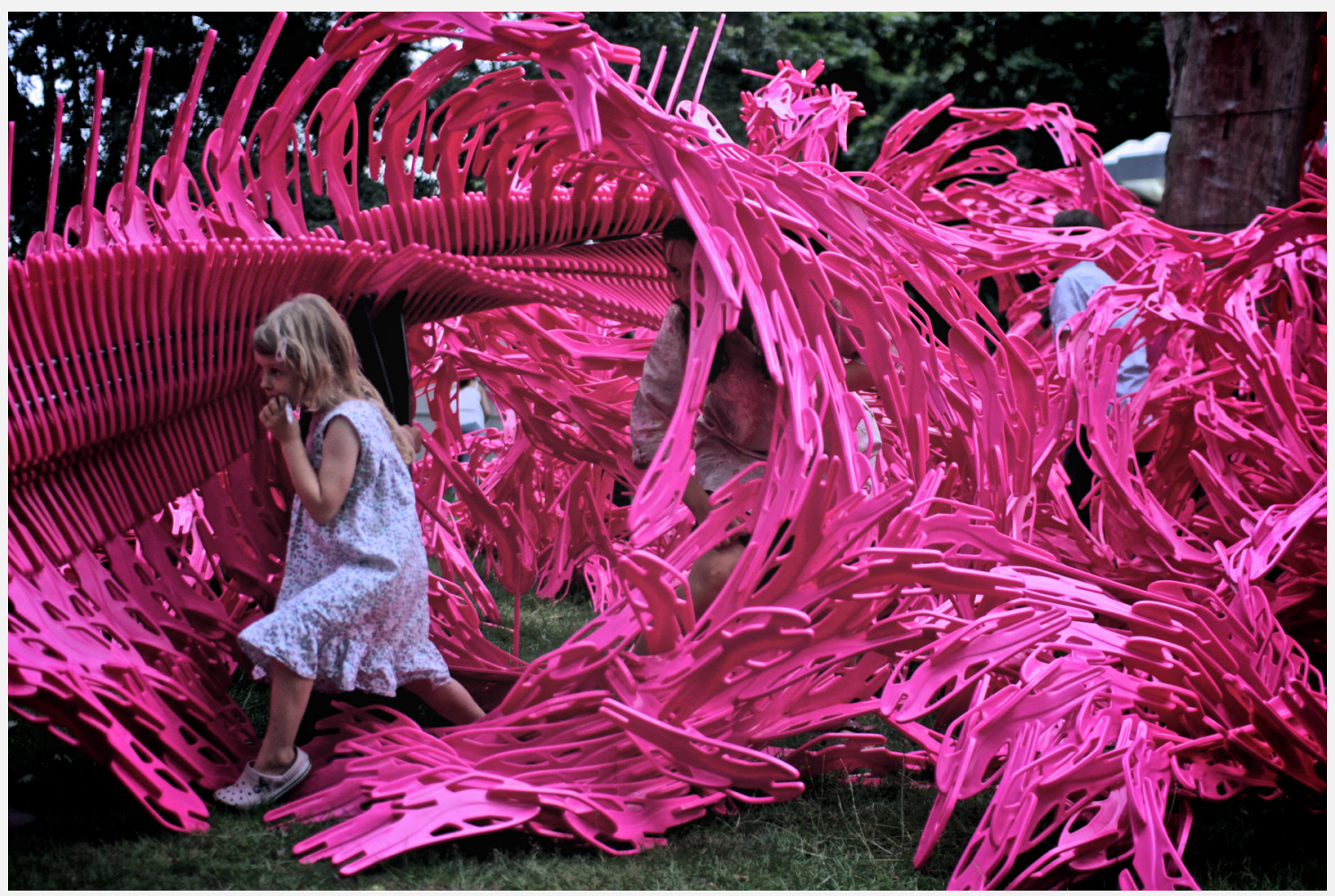

Figure 97. Bloom 


\section{Seeds}

The planned group build never materialized due to lack of participation. However, there was a key instance during the construction of the visual screen, where it was questioned whether it was being assembled correctly. It is worth mentioning again that the system is fairly robust and able to self-organize to optimize its form. Invitation to formally engage is often met with an initial hesitation. Removing such barriers to participation will allow an architecture that is more inclusive and expressive of user needs. The question highlights a few key points: 1) The authority to design or build has been limited to experts. 2) Desire for specificity. 3) A lack of trust in a seemingly disordered formation. 


\section{Case Study: Spiculation - Léopold Lambert, 2008}

Spicules is conceived as a malleable material which can be transformed at any moment by anybody. Léopold Lambert states that once they are manufactured and let on site the system becomes democratic as it belongs to everyone and everyone is free to act on it (2014). Lambert envisioned different applications, from barricades, to apartment extensions, homeless shelters, to theatres (Figure 98). His intention seems to be one of political—its accessibility to all is a statement of individual agency and power that attempts to subvert established powers through spatial or territorial interventions.
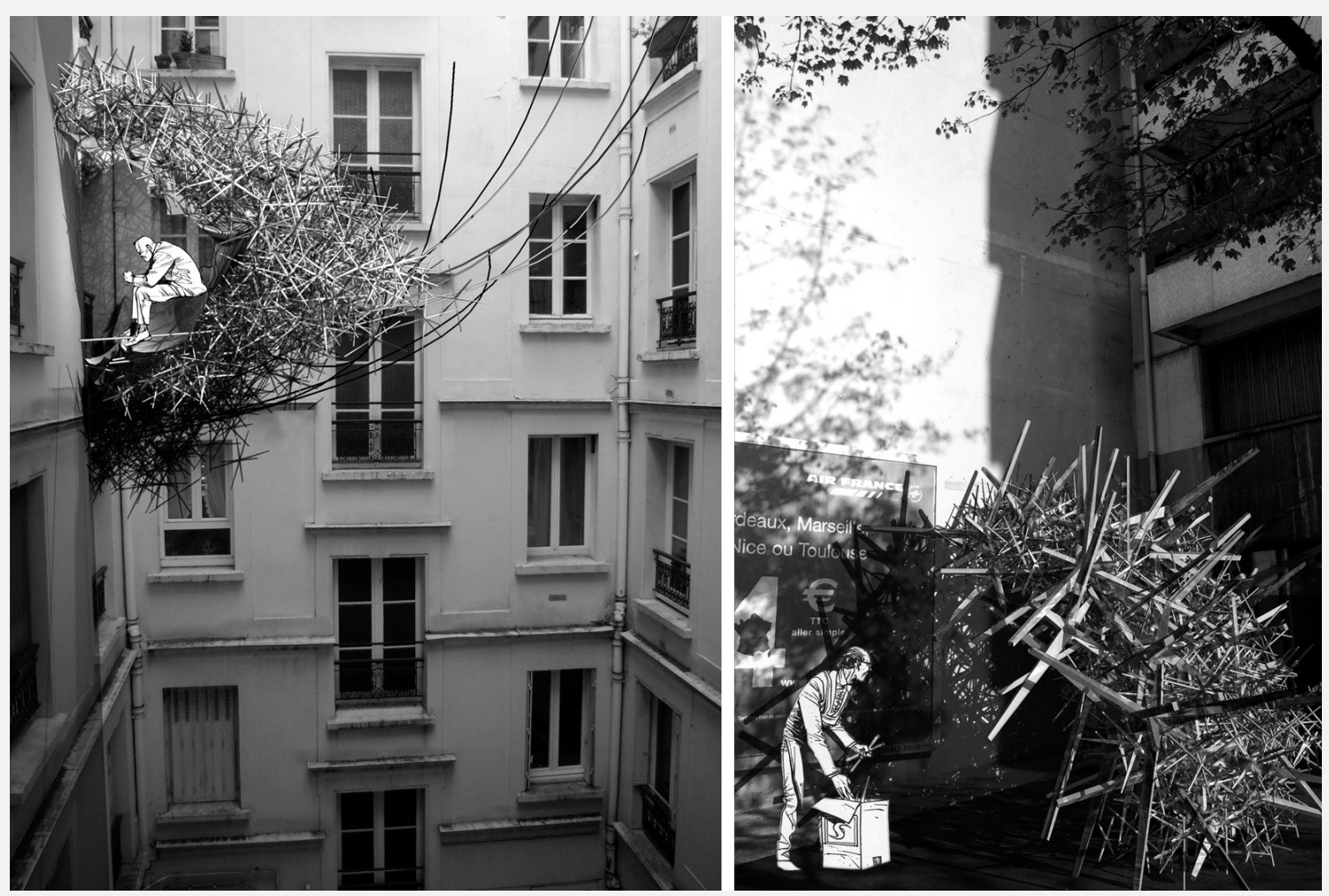

Figure 98. Examples of Spicule appropriation 


\title{
Design Exploration 4: JaXX
}

\author{
March 2016
}

Following the immense level of interaction observed and positive feedback received from the Winter Station exhibition, it prompted a different look at the system's applications. Recalling some of the various uses and objects that had been produced while working with the material encouraged an exploration of the system at smaller scale applications — one suitable for the home as a type of everyday product or construction toy (Figure 99).

\section{Spatial Module}

Stepping back form the idea of a full construction system, JaXX was positioned as a simple spatial module, a multifunctional space making component, capable of quickly assembling into various structural forms and adapt to diverse applications. When coupled with additional accessories such as a tabletop or lamp it becomes a form of modular system that could be furniture, but could also be a divider or trellis in different scenarios (Figure 94). It was promoted as a flexible building block and open to user interpretation (Figure 100).

The module was based on the shock cord development which has been shown to allow disassembly with greater ease - a quality equally relevant in the context of this design problem. Similar challenges of transport and storage from Flow led to greater considerations on the storing mechanism and packaging. Framing the system as a consumer product, rather than a construction system, required a different set of sensitivities. For example, material choice and build quality took on a different level of importance in this new context due to its more immediate connection with the user. Furthermore, the formation of a business plan to a greater appreciation of market factors and economics. 


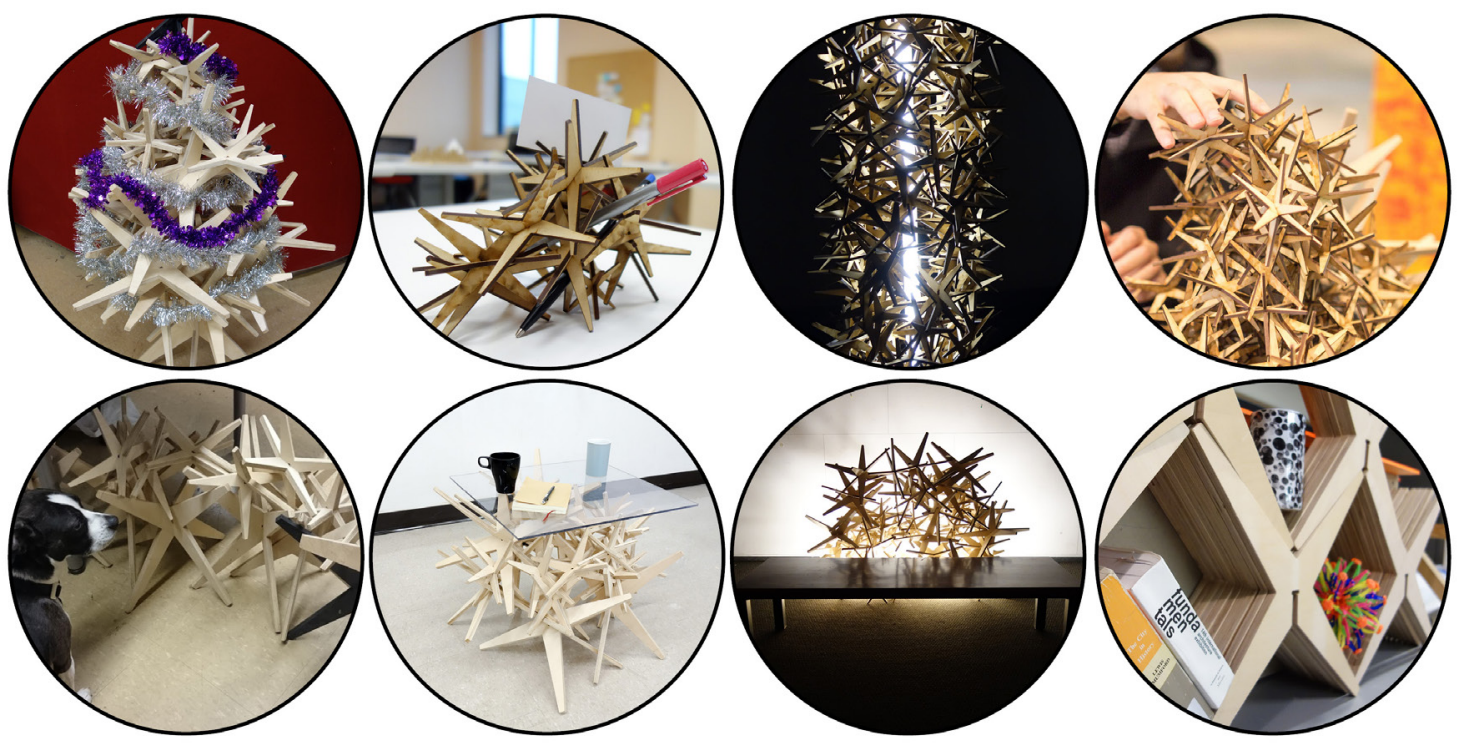

Figure 99. JaXX employed in various applications

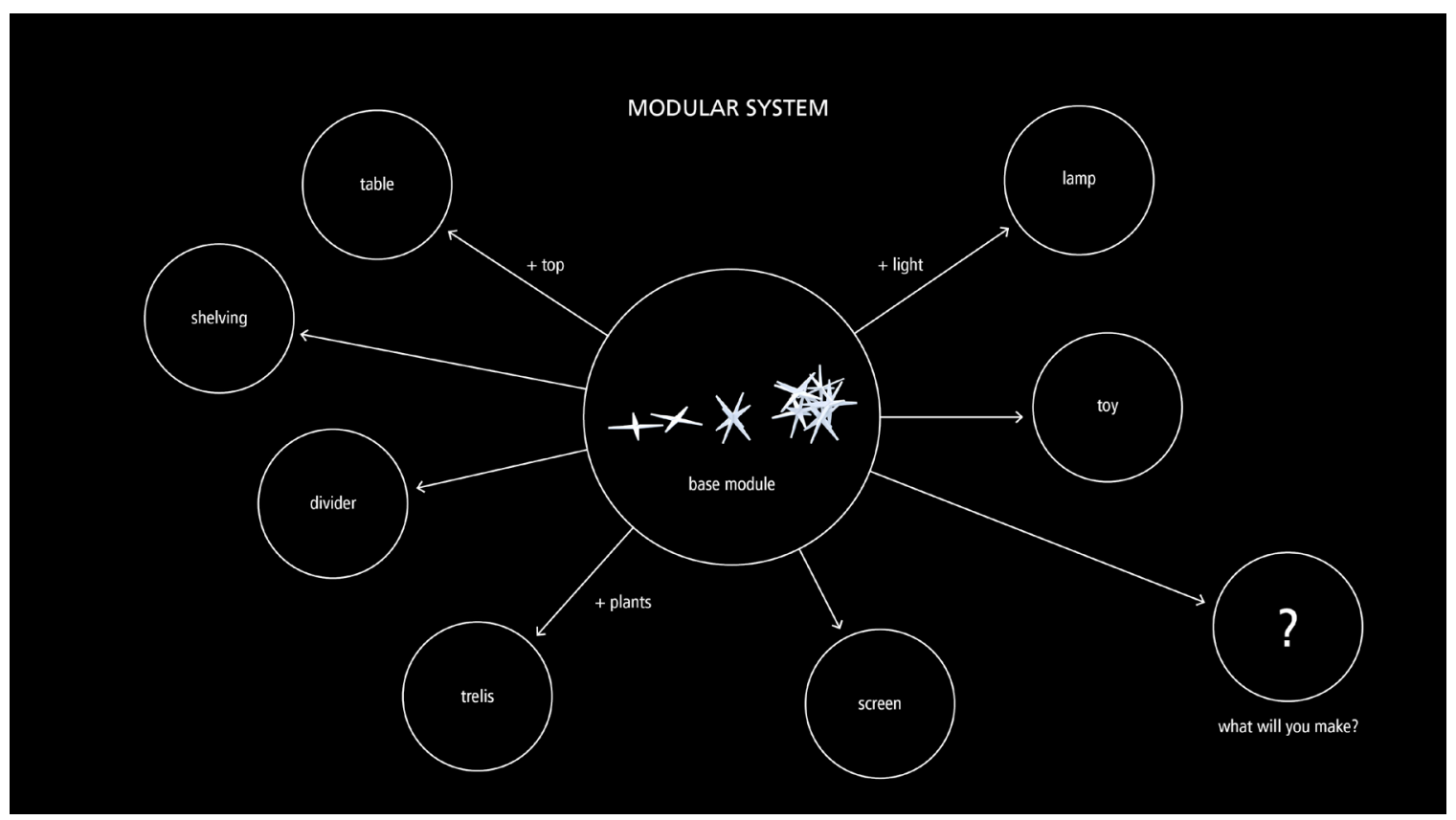

Figure 100. Diagram of modular system concept

150 Architecture at the Edge of Chaos 


\section{Construction Toy}

The other main application proposed involved the smaller $10 \mathrm{~cm}$, components. Throughout this investigation the material has exhibited, an ability to engage people's primal inclination to stack objects together revealing its potential to be simply a construction toy (think of children's building blocks.) In its current form with its spiky property it could function well for adults as a desk toy for relieving stress. In fact, its stress relieving potential had been mentioned numerous times and was once compared to the therapeutic capabilities of knife throwing in reference to the feeling when one of the thrown components "stick" to the assembled pile.

This system, when compared to many other construction toys such as the popular Lego, is different in that unlike Lego which has discrete connections and thus limited configurations, the designed granular system is an "analog" material where the pieces can come together in an infinite number of ways. A "digital" material would characterize many modular type of architecture/construction and to a large extent Lego today, where components come together in specific ways. This type of specificity imposes certain limits. On the other hand, like a generic building block, a granular system based construction toy allows for greater freedom and encourages a type of freeform, sculptural play that acutely takes into account the dynamics of gravity and forces (Figure 101). "Rudolf Steiner, one of the most influential of play theorists, believed that only the vaguest sense of reality should be designed into a toy so that as much room is left for the imagination as possible" (Heathcote, 2013)" wrote Frank Lloyd Wright (1867-1959. The simple-shaped module of JaXX can be argued to achieve that.

It is worth briefly mentioning the close relationship between construction toys and architecture. For one, it is not surprising that building sets have influenced many architects. Frank Lloyd Wright had acknowledge the influence Froebel Blocks had on him as a child. His son would go on to become an architect as well and develop Lincoln Logs, a construction toy based on the Japanese method of building foundations with notched logs. Norman Foster is said to have been "touched by Meccano" (Foster as cited by Heathcote, 2013)” wrote Frank Lloyd Wright (1867-1959. Women, such as Ray Eames and Anne Tyng, also had a large part in designing construction toys (Heathcote, 2013)" wrote Frank Lloyd Wright (1867-1959. The toys reflected the sentiments and concerns of their time.

\section{(Refer to Appendix E for additional images.)}




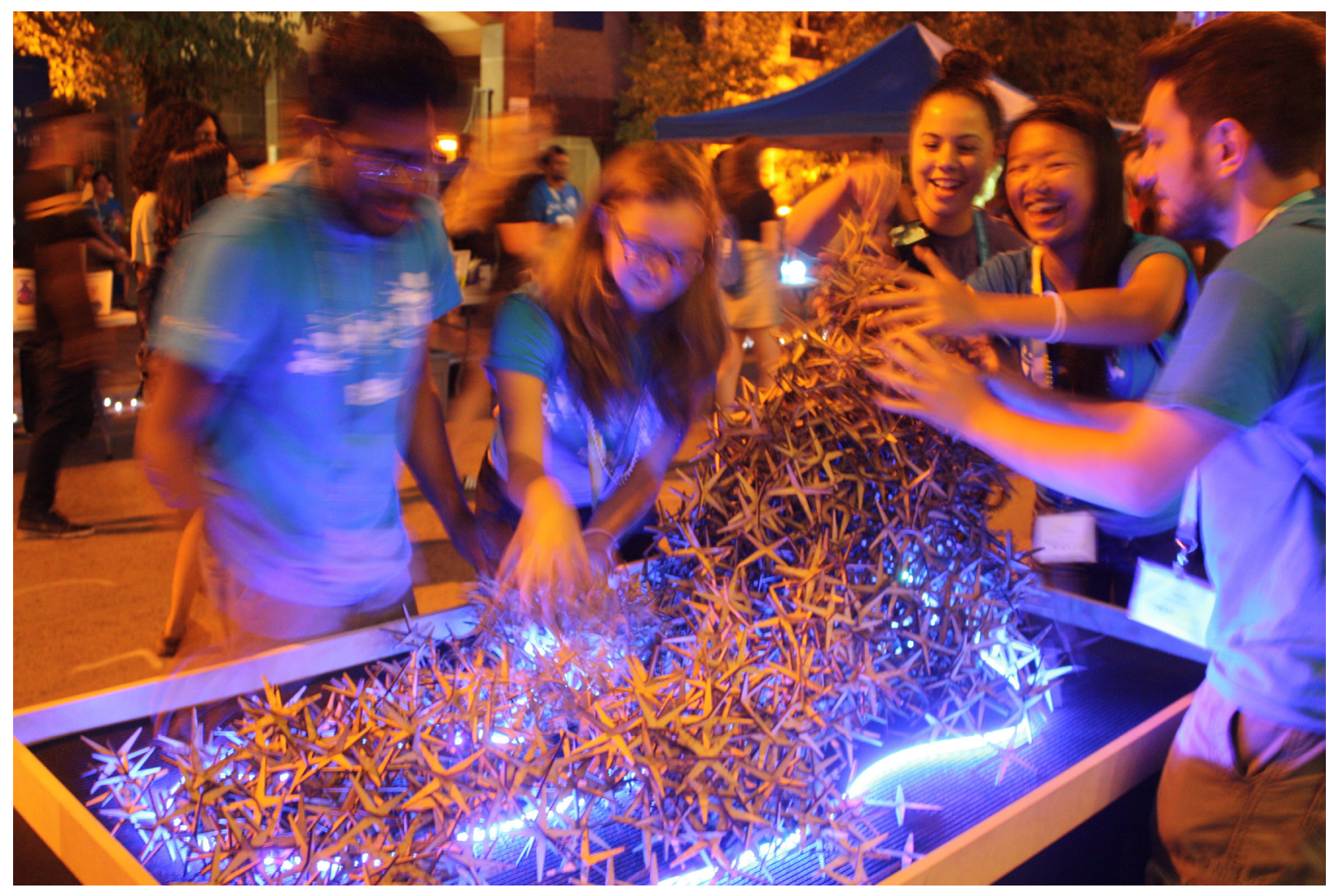

Figure 101. Its generic module is exploited to stimulate creativity and play as a constructional toy concept

152 Architecture at the Edge of Chaos 


\section{Case Study: Tsumiki and Chidori - Kengo Kuma}

The relationship it seems, is especially strong in Japanese architecture and is evident in particular with the works by architect Kengo Kuma, whose designs have adapted constructional techniques from traditional Japanese toys and also inspired new toys. Tsumiki ("wooden blocks" in Japanese) are simple V-shaped wooden blocks designed by Kuma. Tsumiki pieces are made of Japanese cedar, measure about 4" $x 4$ " and contain triangular notches in the legs allow the blocks wedge together. They can be stacked and arranged in a variety of ways to create unique formations (Figure 102). "They're natural in material, spatially economical, and relentlessly simple," was one description of them (Rhodes, 2016).

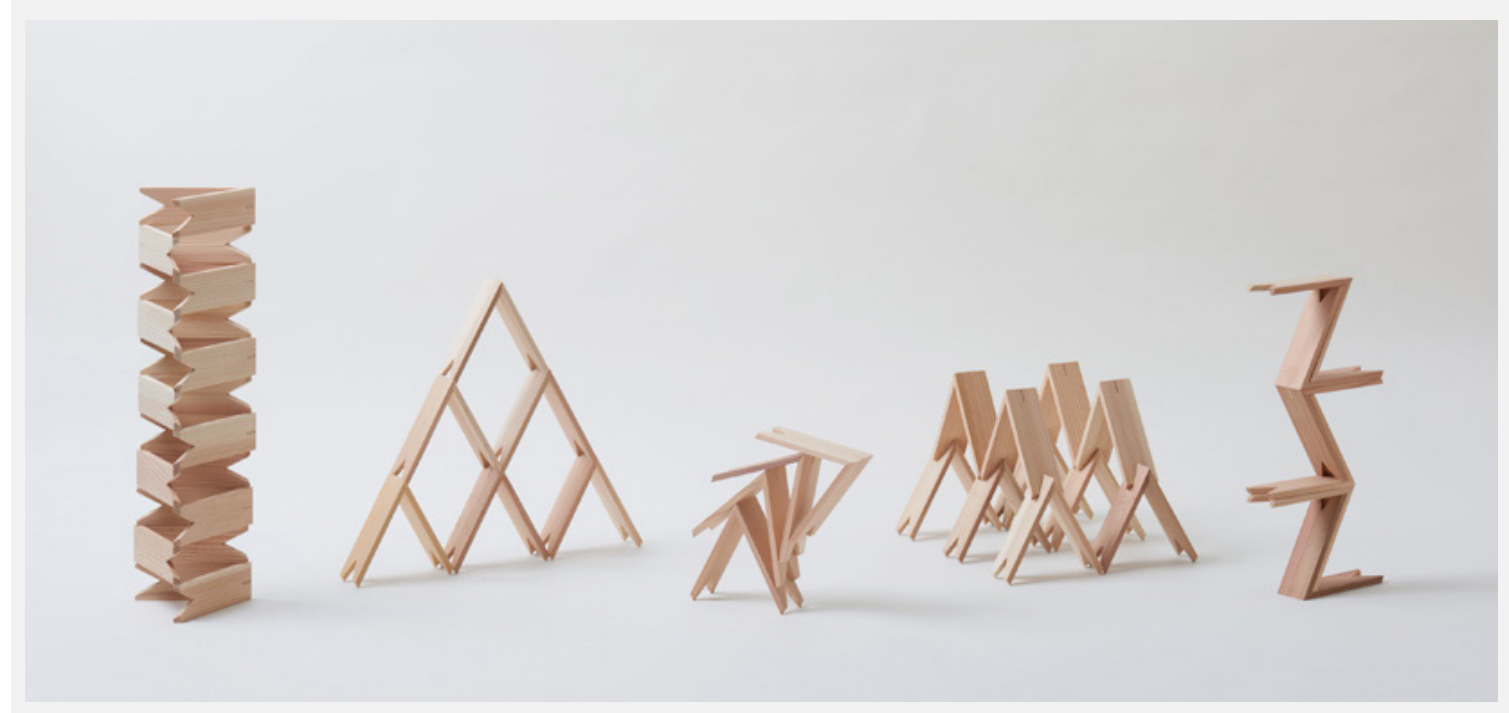

Figure 102. Tsumiki toy

Kuma used the same concept to design a temporary Tsumiki installation in Tokyo's midtown park (Figure 103). In addition to a pyramidal pavilion, individual pieces were arranged in a semi-circle, varying in scale from large to small and encouraged visitors to interact with the individual modules. 


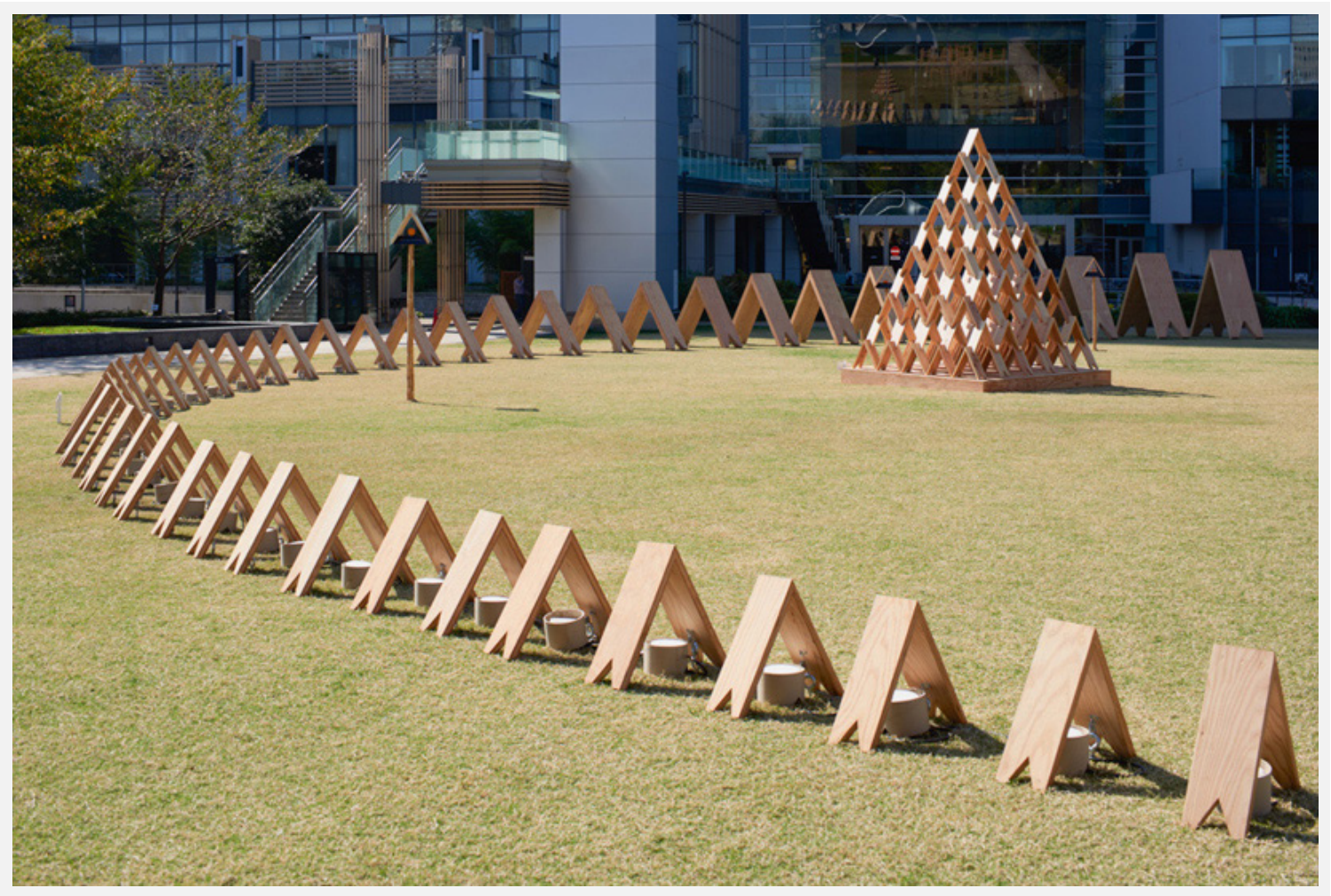

Figure 103. Tsumiki as installation

In another example, the architecture for the GC Prostho Museum Research Center originates from the system of an old Japanese toy called Chidori ("GC Prostho Museum Research Center / Kengo Kuma \& Associates," 2012). It is an assembly of wooden sticks with unique joints that can locked together without any fasteners (Figure 104). This concept has been employed for furniture and the toy even adapted to large structures such as the Research Centre (Figure 105 and Figure 106). The architect states:

[The] structural engineer for the project, conducted a compressive and flexure test to check the strength of this system, and verified that even the device of a toy could be adapted to 'big' buildings. This architecture shows the possibility of creating a universe by combining small units like toys with your own hands. We worked on the project in the hope that the era of machine-made architectures would be over, and human beings would build them again by themselves. (“GC Prostho Museum Research Center / Kengo Kuma \& Associates,” 2012) 


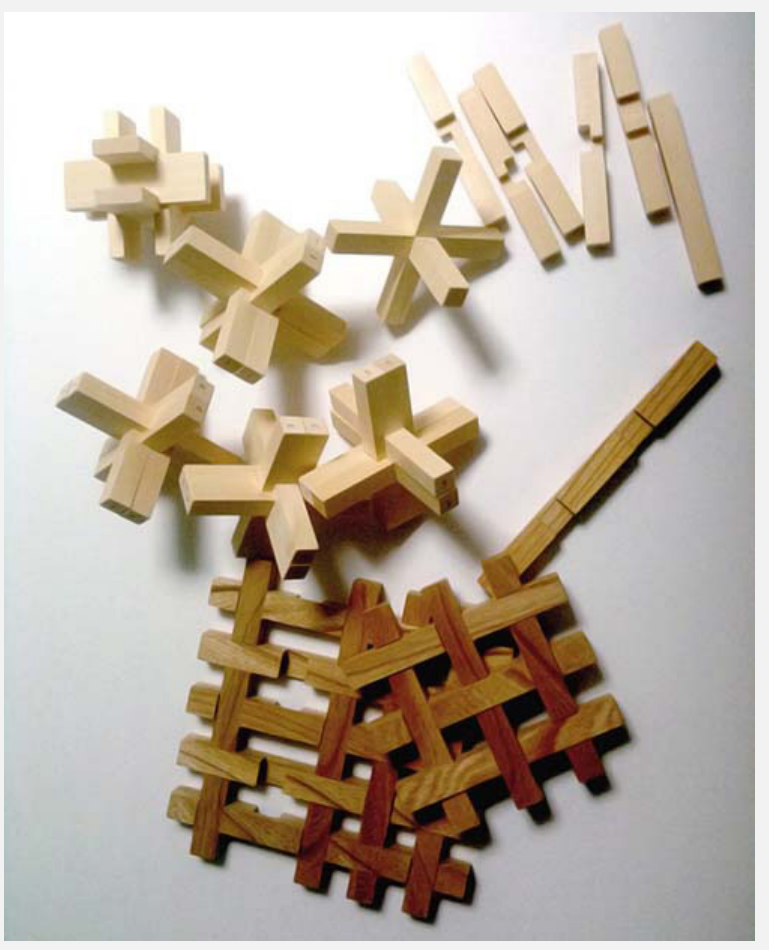

Figure 104. Chidori toys

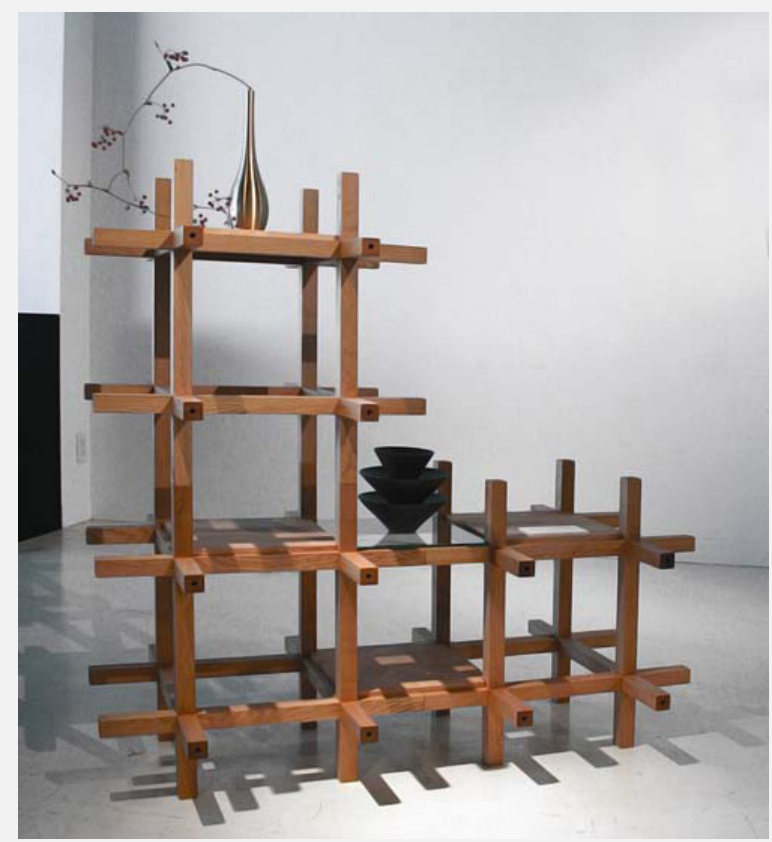

Figure 105. Chidori 


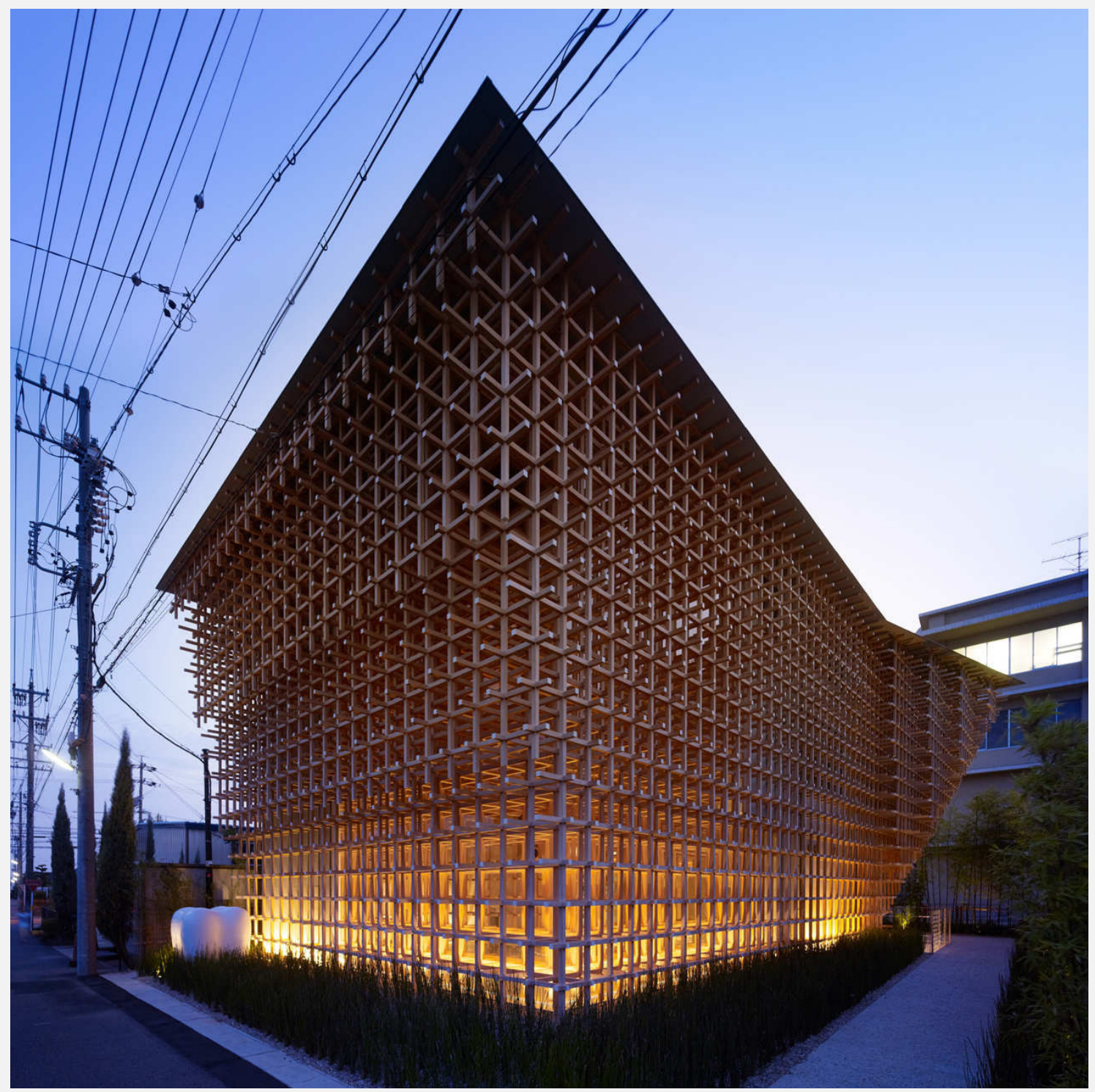

Figure 106. GC Prostho Museum Research Center 


\section{Summary}

Great public interest have been received each time it has been presented to the public. People are always eager to contribute their own ideas and interpretations which speaks to the different types of user creativity outlined by Hill. Stepping back from the macro architecture/ construction scale allowed for a heightened sensibility surrounding the relationship between user and material which will in turn inform a more user accessible material. A simple module is important in this respect. A focus on the user necessarily becomes important due to the system's inherent participatory quality. The installation at the beach could be said to have become a toy-its ever changing form becoming a source of exploration and play. The connection between architecture and play is also a concept that goes back to New Babylon discussed earlier. 


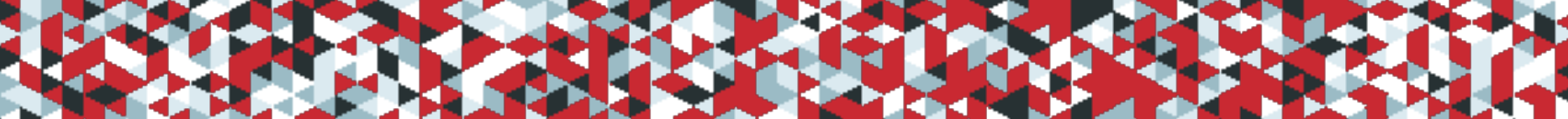

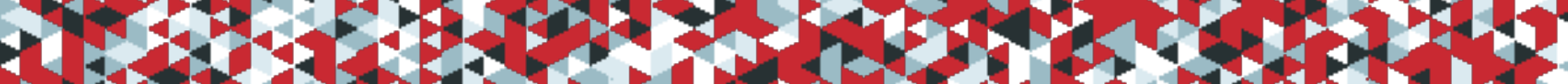

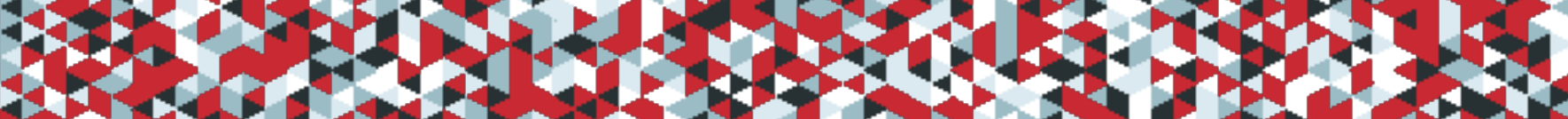

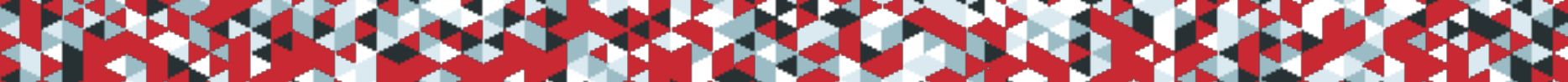

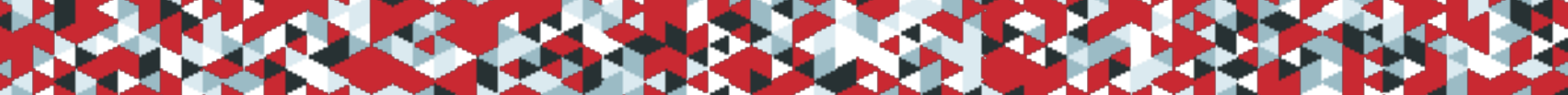

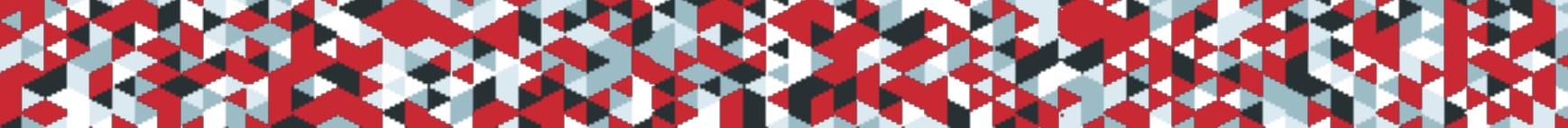

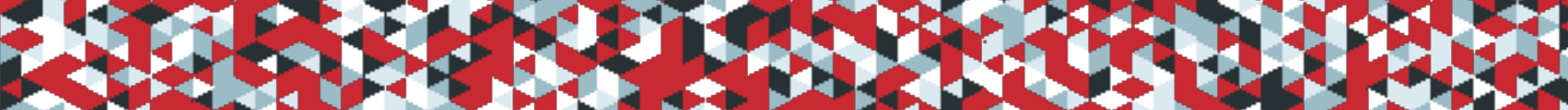

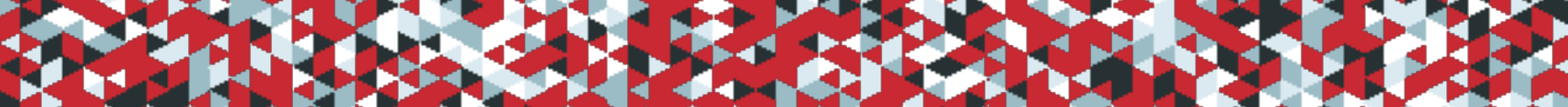

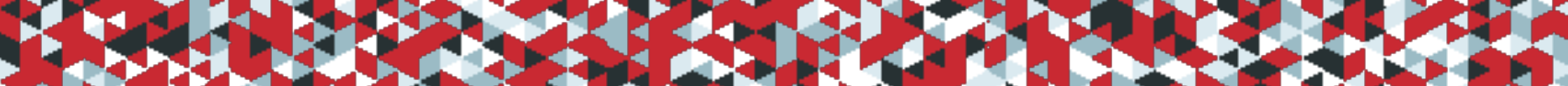

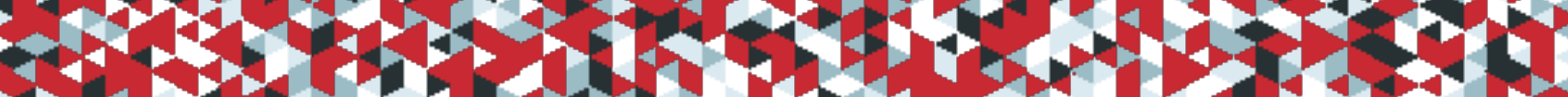
(1)

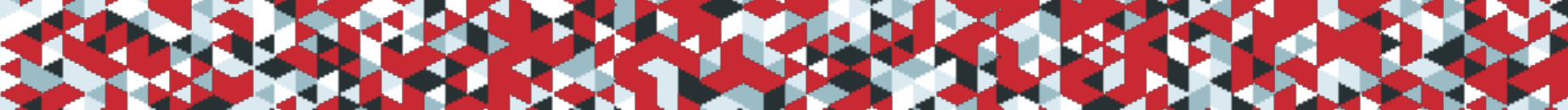

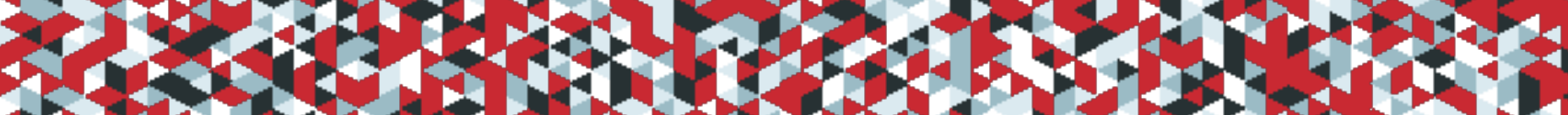

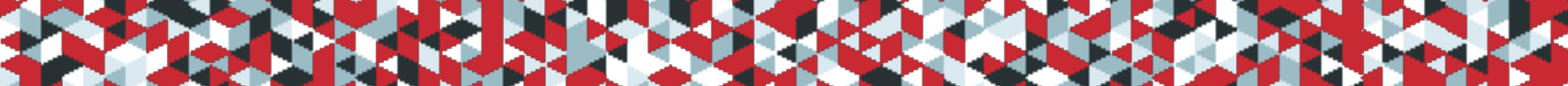

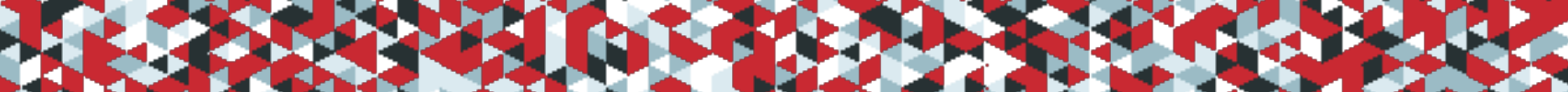

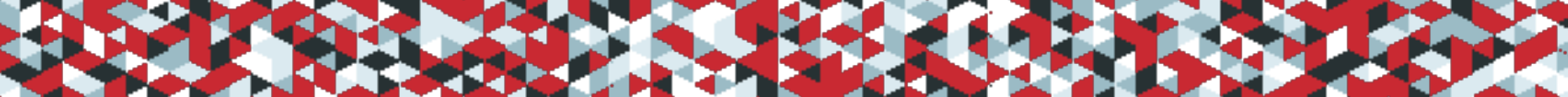

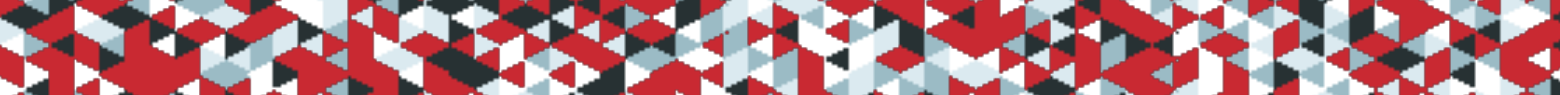

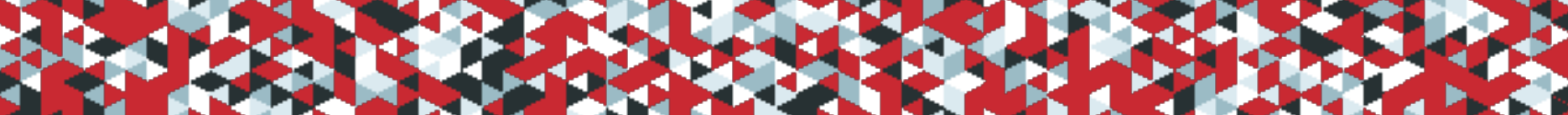

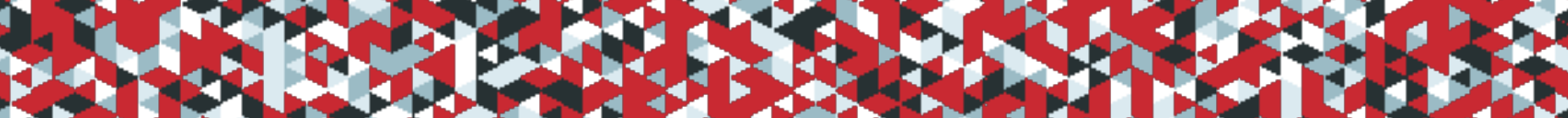

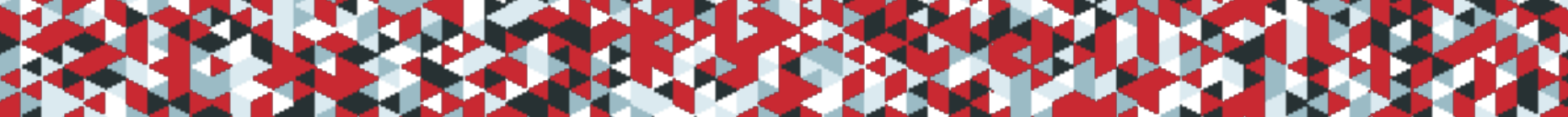

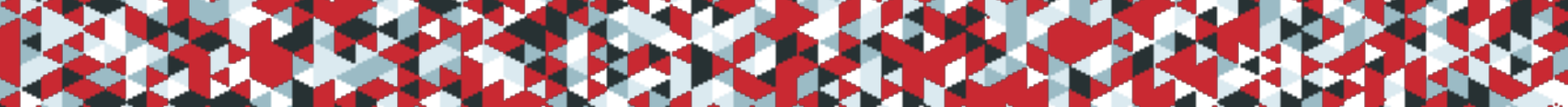

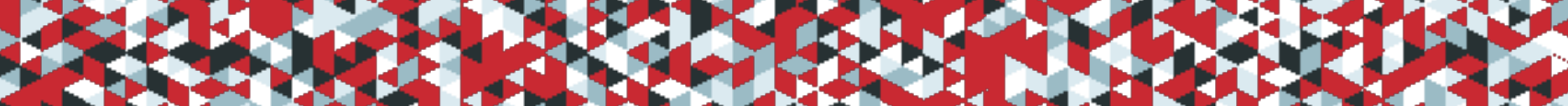

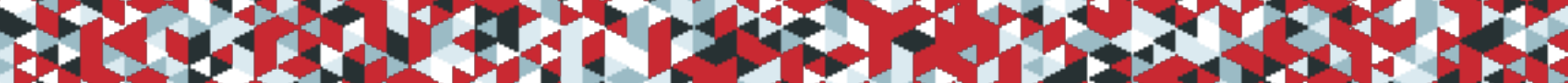

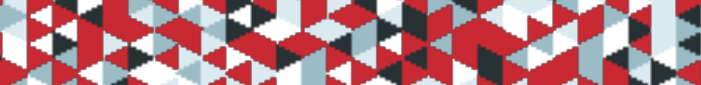
(1) 


\section{PART 5: REFLECTION}

"architecture... embodies chaos, because the process of design is exposed to multiple and complex factors generated by human thought and other external parameters, and because the outcome-the building itself - is not finite but continually in interaction with the indeterminacies of experience and environment." 


\section{Research}

This was a design-research project and thesis that brought together a broad range of discourses, methodologies, technologies and practices and hopes to inform new models and possibilities for architectural research and exploration. Granular material systems radically shifts the boundaries of what we call architecture challenging fundamental assumptions of what the components of architecture are, and in doing so assumptions of how architecture should look and perform. It provides a lens through which to advance and consider a new paradigm of thinking about change, material and structure in architecture. It confronts conventional notions of design and process in inviting human and material agency.

Materially it has demonstrated innate intelligence. Harnessing uncertainty and chance in its very processes, it is malleable, strong and resilient. Its loose bonds allow great sensitivity and reception to change. Through physical experiments and observation the approach is still based largely on trial and error. Granular architecture advances into territory where little empirical knowledge exists and design rules will need to be developed. Going forward, there clearly will be a tension between, on the one hand, the system's apparent simplicity, and on the other hand, new structural and aesthetic possibilities achieved through sophisticated technology. Computation, material science, and robotics are some of the complementary fields that have begun to examine the technology. The granular material architect will need to become an interdisciplinary practitioner.

The designed granular system operates at a multiplicity of levels, evolving beyond just the physical material level to engage the immaterial—-the social and conceptual—structures of architecture. The exploration itself, in keeping with the material's logic, has also been non-linear, taking many paths and turns in the process of discovery. What was the most exciting revelation here, was the material's extraordinary social component. For the first time, its public reception and engagement was thoroughly documented, allowing for new discussions surrounding the social dimensions of this research. Agency, participation, and creative freedom, are the values revealed to underpin this material concept. For this I hope that technical research maintain the ideals of simplicity and accessibility in order to preserve these values. 


\section{Design}

Capturing both the information of the environment and users, the material begins to move towards a truly adaptive architecture. The question it begs however, is what becomes of design and the role of the architect when apparent randomness, complexity and self-organization govern such systems? A new diagram of the role of the user, the architect and the technological platform in the way design is conceived and realized is required.

It comes down to the concept of control, and agency. The user, who traditionally has relatively very restricted control over the domain of space is now provided the means through the accessibility and simplicity of an architectural granular material system, to engage and participate in architecture in a more robust way and to make it their own. The architect will undoubtedly be concerned over the loss of authority, but if the architect represents order and the user (and other external forces), the potential for chaos, what is desired is simply a better balance-the fruitful edge of chaos. Avoiding centralization of control from the top requires that architects relinquish complete control and take a soft/ light approach assuming a background role which, elevates social empowerment. Their task will be to focus on local components and its relationships to influence overall global behaviour rather than impose specific form that ignores the factors of time, the environment, and users. "The role of the architect... is not so much to design a building or city as to catalyze them: to act that they may evolve." (Gordon Pask as cited in Frazer, 1995, p. 7 Foreword)

Design, with granular material technology reconsiders the assumption that design is associated with order, purpose or intention which can only arise out of a sentient designer. There are clear external forces equally capable of contributing to design. Nature, self-organization, and chance all possess the creative potential. Furthermore, the notion that high performance is typically associated with regularity and order is reversed in the context of adaptability with granular materials_-ambiguity becomes a strength contributing to robustness and resilience.

Systems of the world will tend to evolve until they are poised at the edge of chaos (threshold of change). They are all intertwined influencing each other in ways that cannot be completely understood. Like a pile of sand, subjected to dynamic forces and ready to collapse or reorganize into a more robust state, the system of architecture finds itself at a similar critical threshold faced with natural and cultural uncertainty. A recognition of uncertainty and its creative potential will be necessary to engage in the real. 



\section{PART 6: UNCERTAIN FUTURES}

"What do you want, Brick?"

— Louis Kahn

(1971) 
A few simple particles coming together can produce a myriad of outcomes; a couple million, could see limitless new realities. During the course of this work, I had my own battles grasping with the concept of not knowing and control. What is this material best suited for? What did the material want to be? In this section potential futures are speculated. It interrogates the ways architecture can reorganize itself to a state of higher complexity, whereby new forms and processes can emerge. Frequently the case with emerging technologies, its greatest applications may yet to be uncovered.

\section{Sites of Chaos - Natural Disaster Relief}

An immense and devastating force of unexpected change is natural disaster. At a time witnessing increased frequency and severity of these events (source), the stakes provides a compelling reason to rethink our architectural responses to disaster. The contemporary scenario of disaster relief-a condition that brings with it instability, uncertainty, urgency, transience, and complexity-would be an ideal situation to consider a developed architectural granular system. Such systems promise to rethink how materials and resources are used, challenging procedures and logistics, and addressing community.

The hope is to shorten response timeframes when it comes to shelter, and challenge inflexible, often "one-size-fits-all" or universal solutions and typologies. A designed granular system, while modular, can be tailored through even small variations to produce maximum diversity to respond to specific conditions. Typical response begins with the provision of temporary tents, followed by movement into transitional housing and finally settling in permanent housing. It is often a very long process which can be quite disruptive. A quick, consistent and incremental solution that is designed to change and adapt with time and situations, unlike current distinct solutions, is proposed by an architectural granular system.

Rebuilding community, through collaboration, will be just as important as rebuilding their homes. The construction system has demonstrated its ability to facilitate engagement and can leverage unskilled labour provided by families. Its accessibility will ensure the greatest reach. 


\section{Earthquake / Tsunami Relief — Japan}

It was demonstrated that granular systems can strengthen to a certain extent with vibration. This can potentially be exploited to accommodate aftershocks of earthquakes. Debris and uneven terrain is of little concern for the system which is able to bridge level differences. A porous configuration allows for drainage in flooding effected areas. Culturally, Japan is well familiar with many design aspects of granular materials with their embrace of its unique forms (via its breakwaters), modularity, and elaborate joinery. Government and other institutions in Japan has been a leader in the open building movement, encouraging and coordinating a number of experimental projects in the country, most of which have been driven by a technically determined agenda (Kendall \& Teicher, 2010, pp. 21-22).

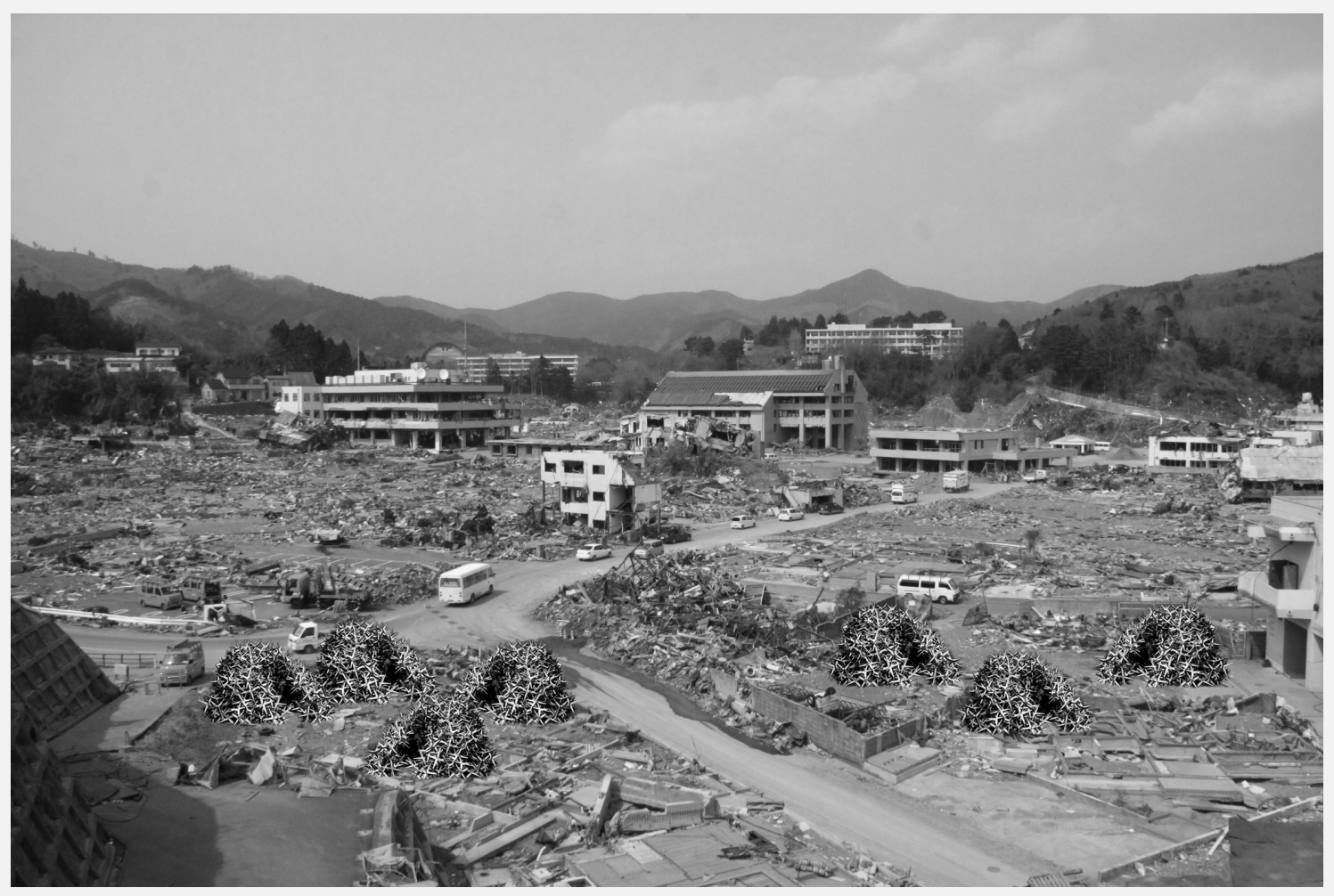

Figure 107. Visualization of material deployed as shelter in disaster region 


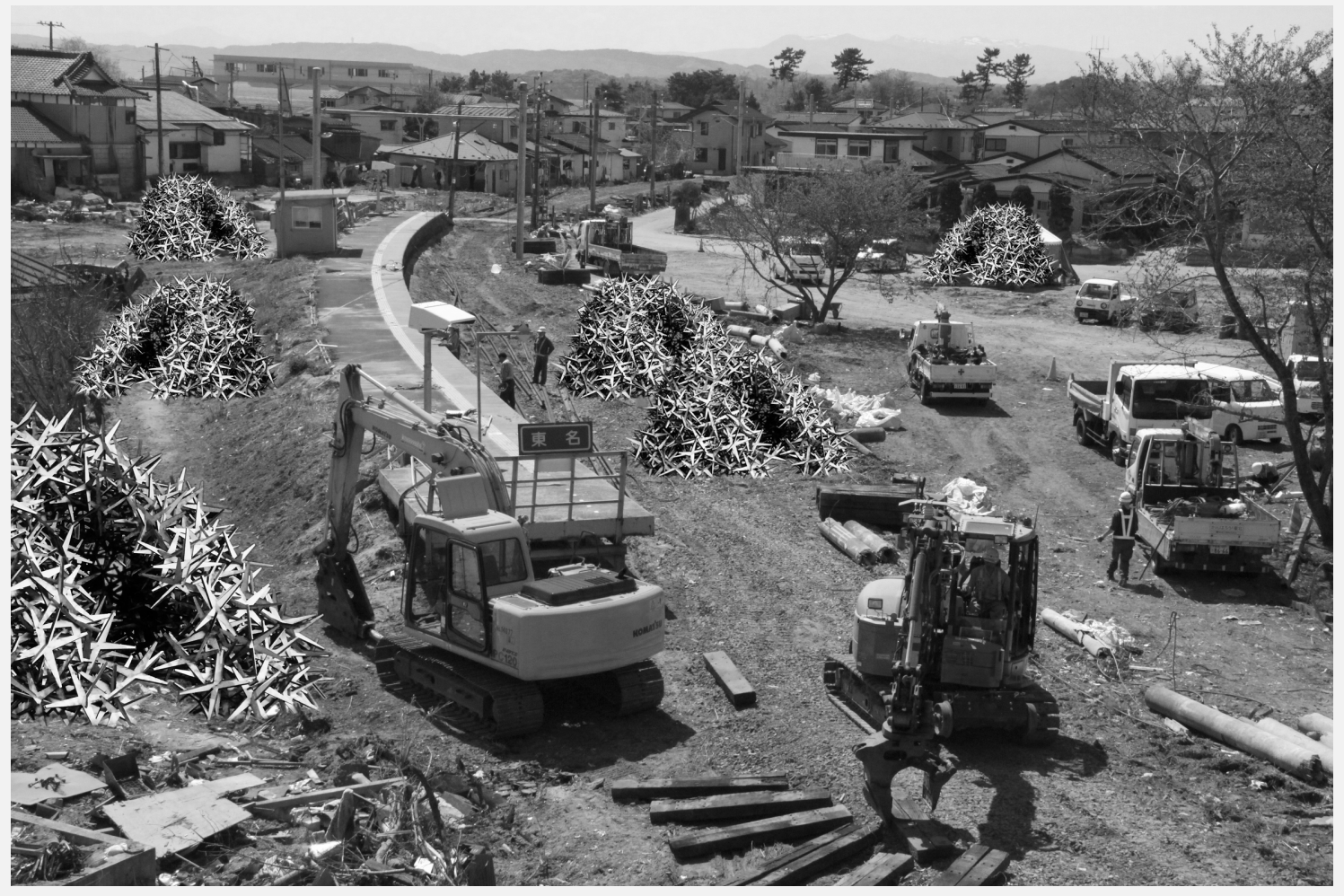

Figure 108. The system is valued for its rapid deployment and ease of construction 


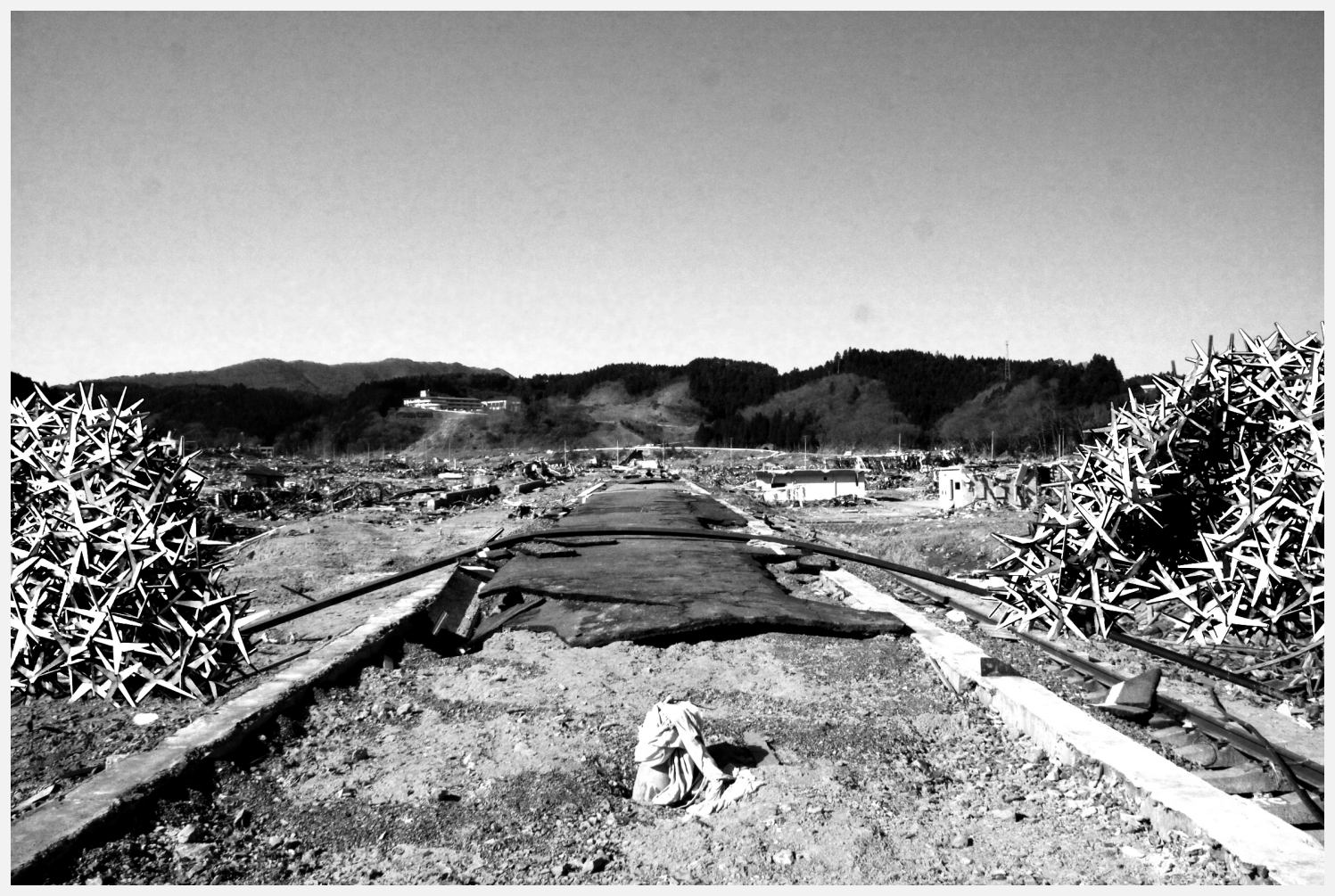

Figure 109. The material is able to adapt to the unknown and harsh conditions 


\section{Defending Freedoms}

Extending from the idea of breakwaters, the concept of a resilient device capable of dissipating forces, conjures up the notions of obstruction, absorption, protection, and defence. Central to this is the material's decentralized structure and resilience. Although performing a bit differently and deployed much more sparsely, Czech Hedgehogs take on a similar caltrop-like form, and were used as anti-tank/ ship, obstacles widely during war (Figure 110). As a device of defence and one that could be assembled by easily by people, depending on the scale, it could act as a barricade or shield from different types of aggression.

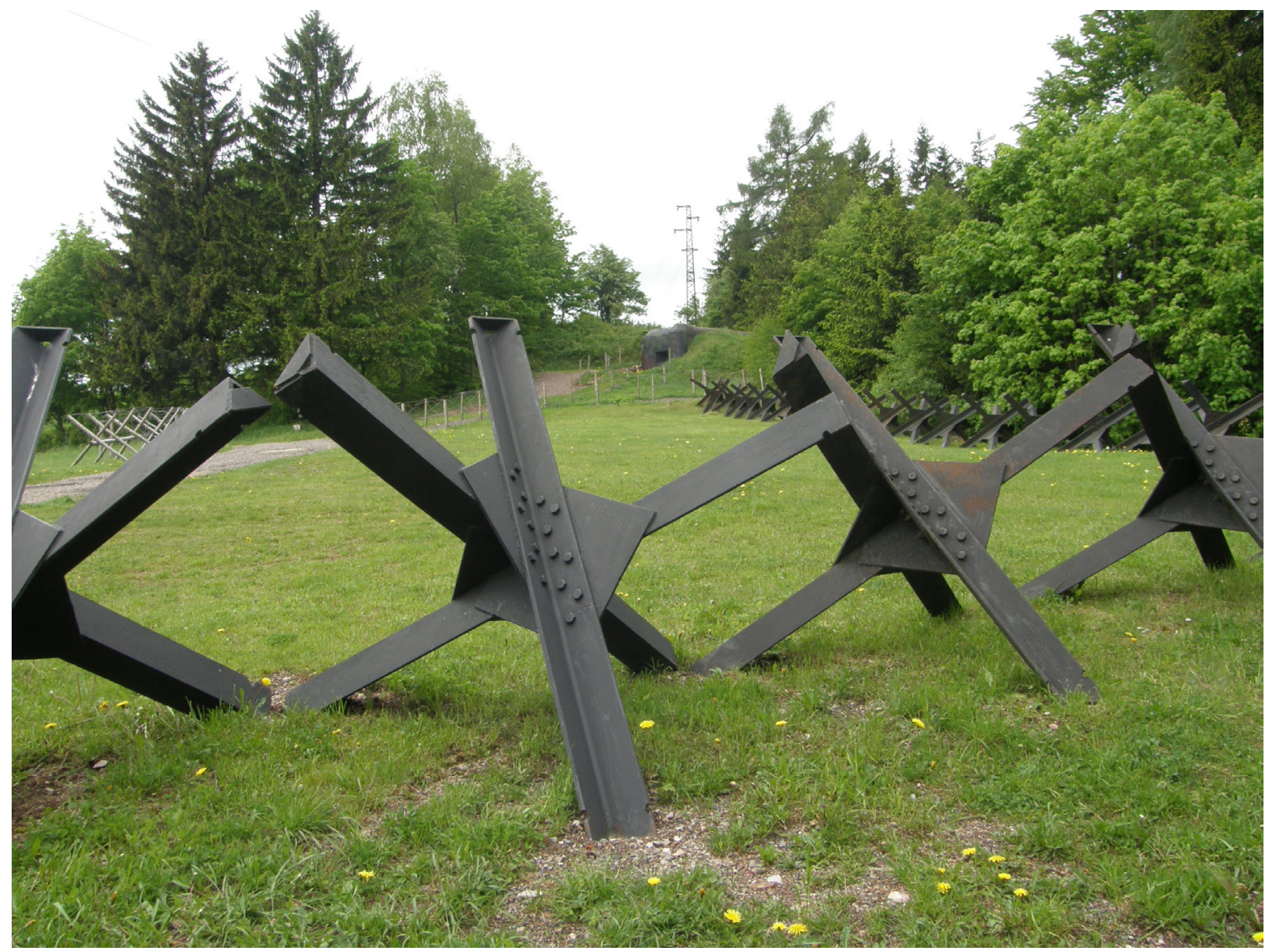

Figure 110. Czech Hedgehogs 


\section{The Coastal Shield—Habitable Infrastructure}

Operating at an urban scale, the particle in this concept is considered as a single residential cell, akin to the idea of modular "capsules" found in the Metabolist movement which fused ideas of mega-structures and organic biological growth. Combining, in this scenario, a mega coastal barrier with an additional purpose, residential, forms a habitable infrastructure. It becomes at once attractive coastal real estate and a protective shield for the inland. The units would be configured essentially into a large berm using container cranes or robotic arms. The multitude of contact points between units will form the basis for a distributed network of movement and servicing resembling the paths of structural force chains.
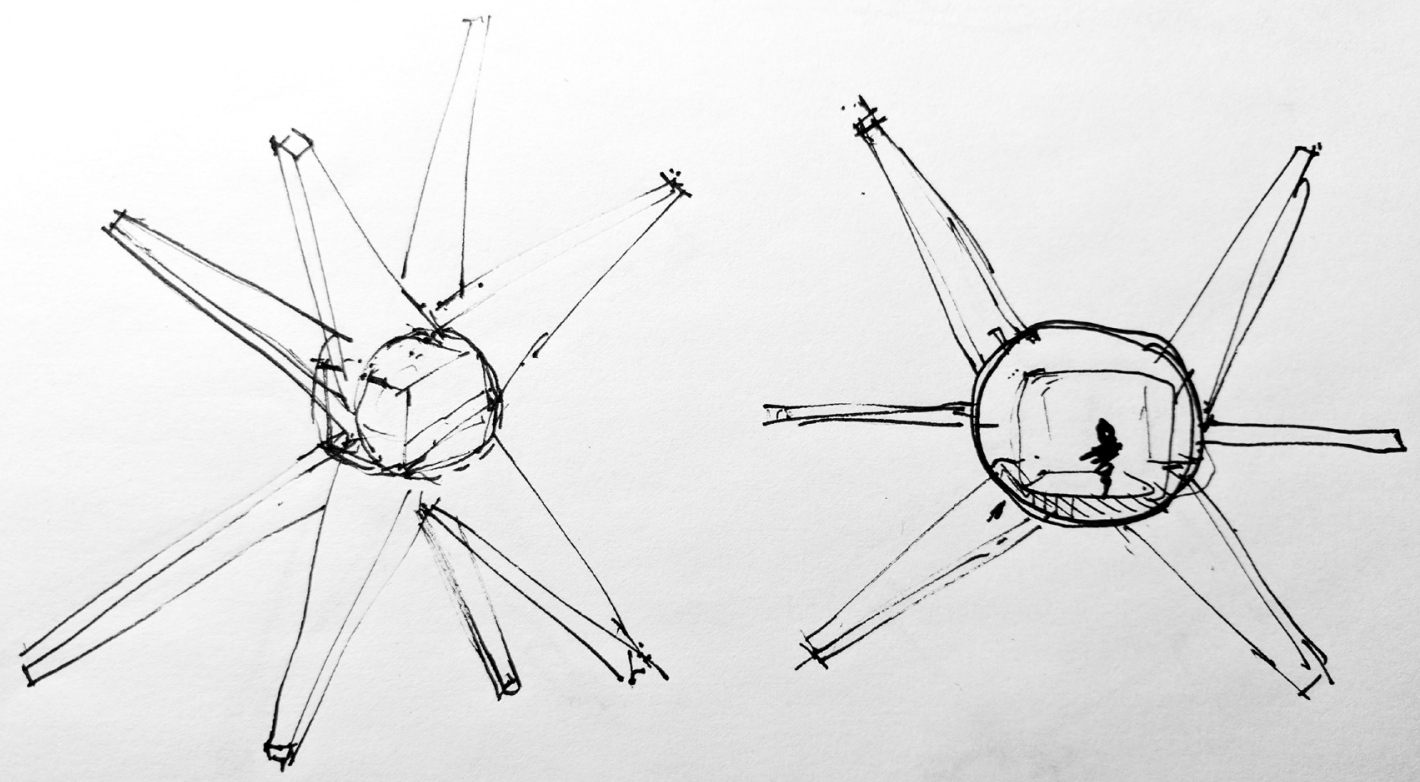

Figure 111. Sketch of capsule concept 


\section{Malleable Matter}

From a material perspective, the discussion surrounding granular materials is a discussion about form and morphology (study of form and structure) which, is relevant to all physical things that we create around us, not only architecture. It would be fascinating to consider an even finer grain iteration of the material, at a molecular or nano-scale that would essentially start to make up matter itself.

\section{Designed Micro Aggregate Substructure}

There are signs the research is heading towards realizing a much more complex type of technology. Taking a step back, the material could be considered in a secondary role. It can become an additive or supplement to other materials. If operating microscopically it can afford many new types of structural properties for other systems giving way to wide practical applications in terms of building science. Consider a robust form of rebar for a new type of flexible concrete-like material. 


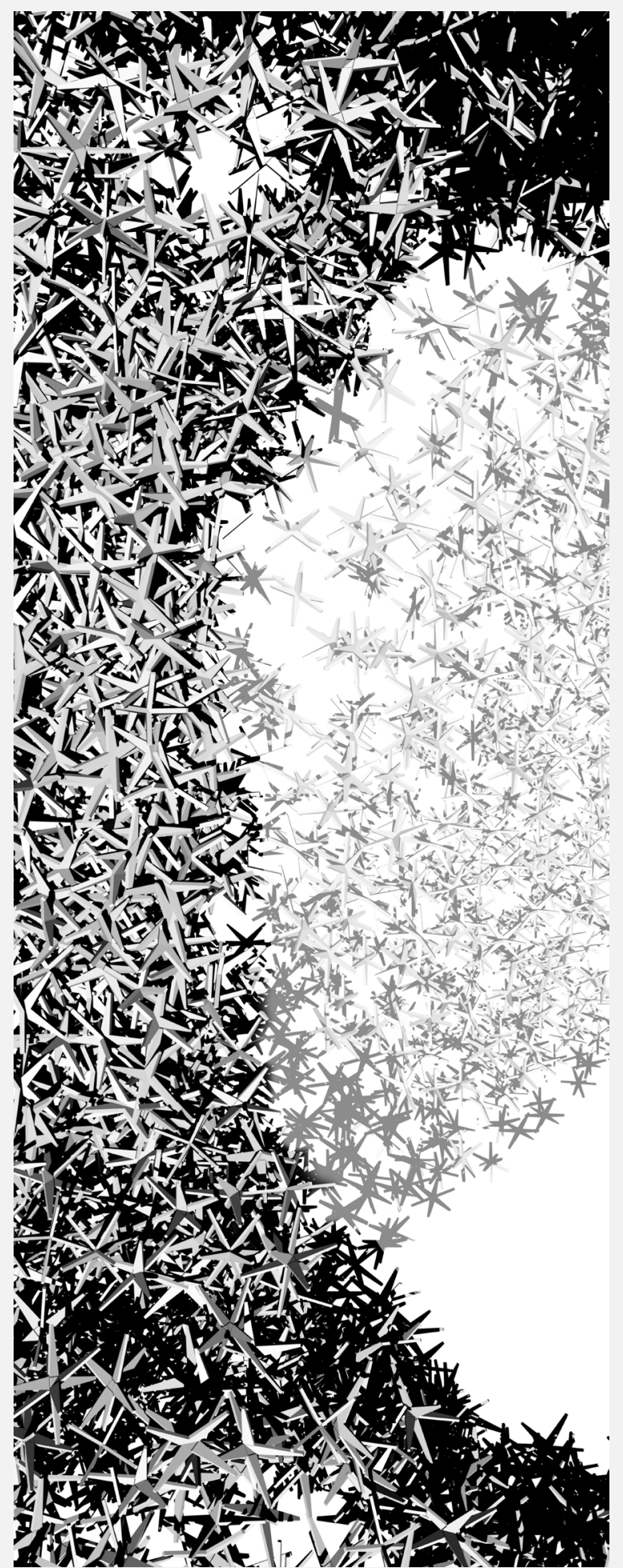

Figure 112. Conceptual visualization matter consisting of designed micro-aggregate substructure 


\section{Creative Chaos}

Life is about discovery. Granular material systems allow the structures of architecture to better engage in this dialog, adapting and growing along with its participants. An architecture at the edge of chaos, despite what it sounds, embraces openness, freedom and the creative potential of chance for those serendipitous moments. As Manolipoulou proposes, "designers, like artists and poets, need to cultivate their capacity for play with chance because it is through playing with chance that we can participate in the real" (2013, p. 213).

\section{SandboXX}

Extending the same spirit of play and creative freedom that Price envisioned in his unbuilt Fun Palace, this derivative concept utilizes the current emerging technologies of architectural granular materials towards a fully interactive and configurable space for all to explore and enjoy. 


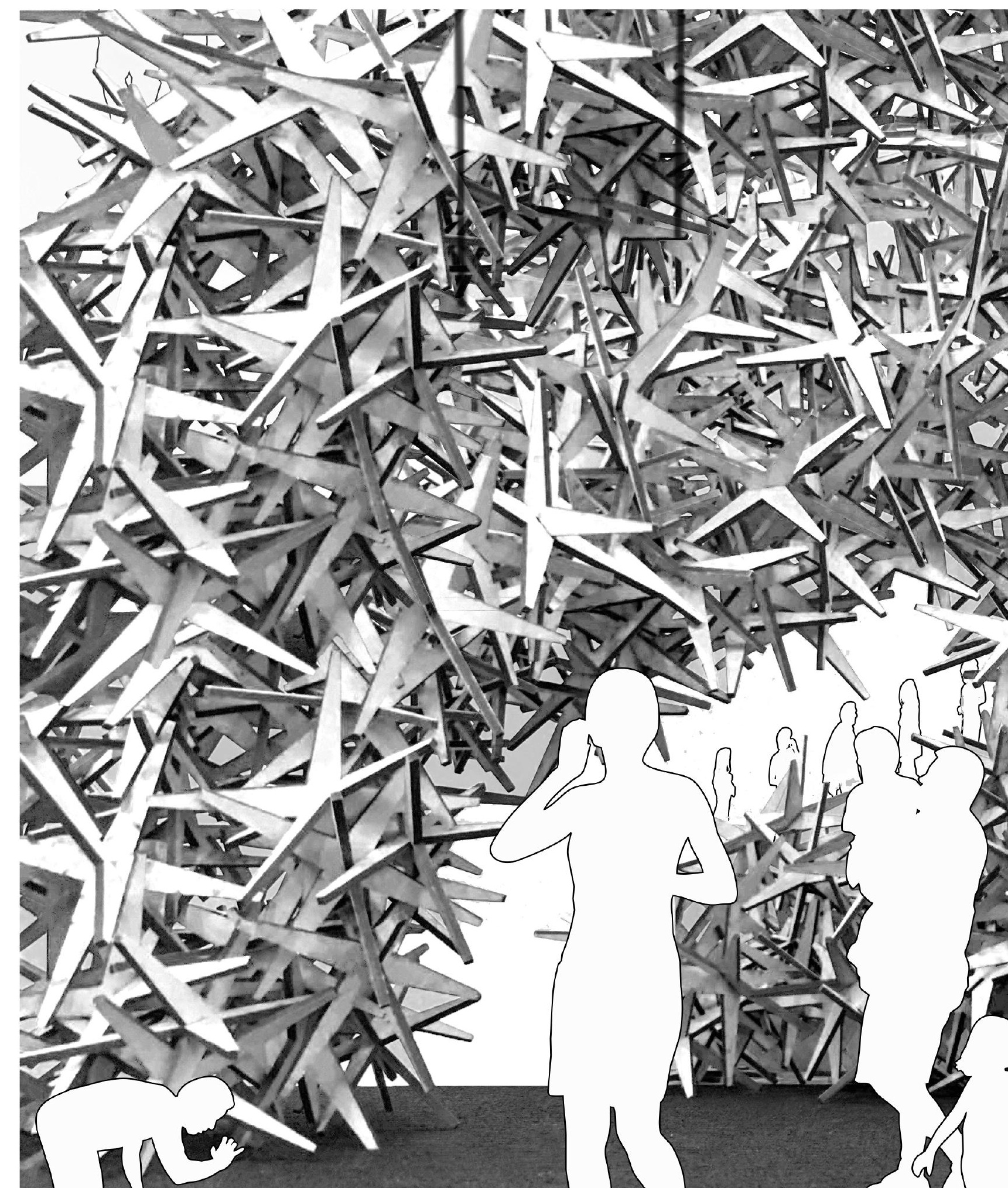

Figure 113. SandboXX interactive environment 
176 Architecture at the Edge of Chaos 


\section{APPENDICES}


Appendix A: Fluid Architecture: Pneumatically informed aggregate system of designed particles

\section{experiment variables}

ELEMENT

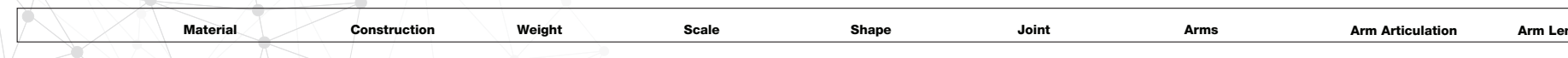

sheet material

mdf

acrylic
rubber coated maf

rubber coated molf
birch multi plywood

birch multi plywood
pvc komatex plastic

pvc komatex paund material
fout

found material
toothpicks

binder coil

moulded
plastic

metal

legend

- large particle

- primary path
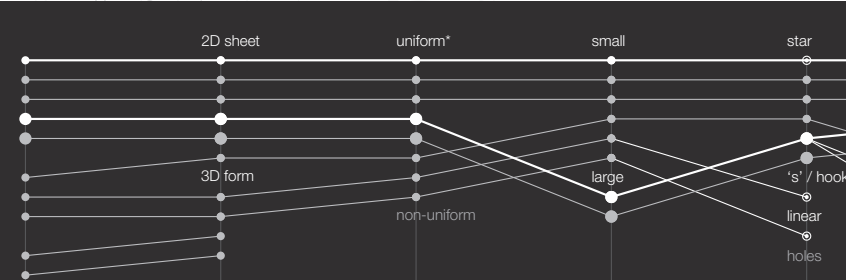

triction slot
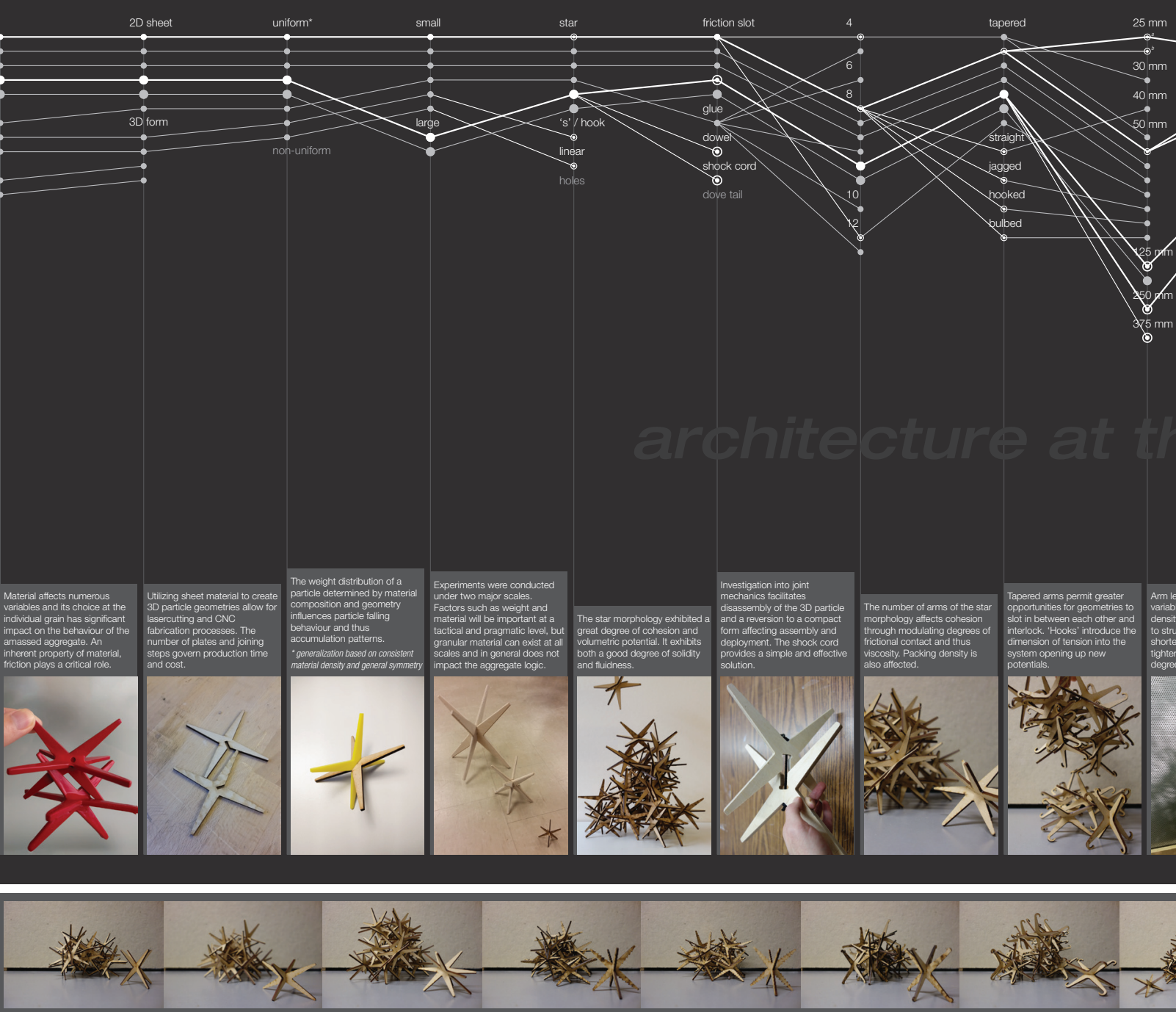

Poster tracking experimental variables

178 Architecture at the Edge of Chaos 
ARRANGEMENT

ENVIRONMENT

igth

Composition

Deposition

Control

Organization

Formwork

Binding

Surface

Loading

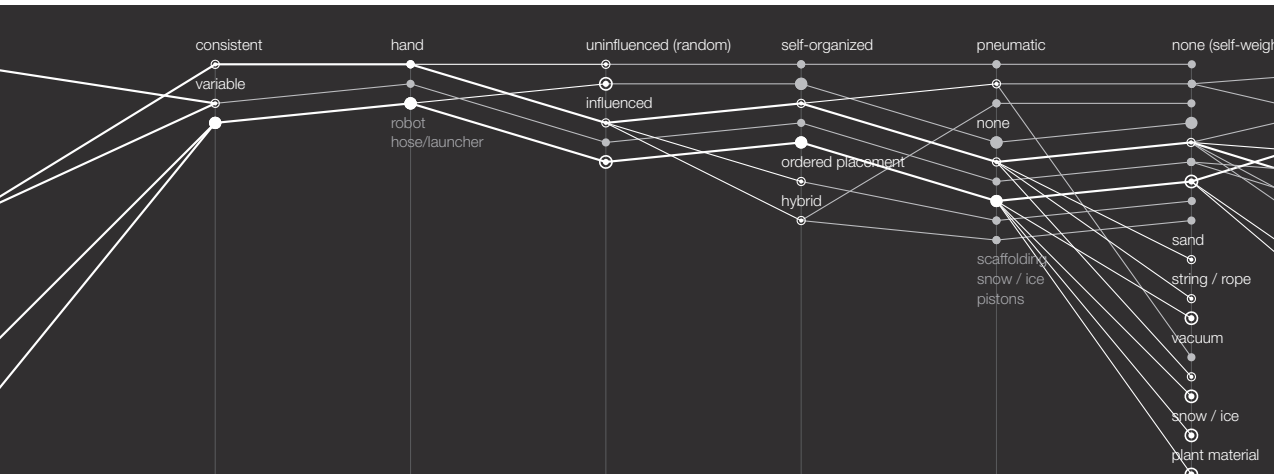

sand $\quad$ gravity only (vertical)

corngated sheet or mat* :

acrylic

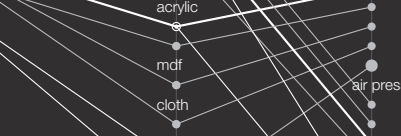

magnetic

external load

ssure / wind (lateral)

grass

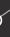

ectiles

ical loading

seismic (shake table)

The strength of the system is its

$\begin{array}{lll}\text { Pneumatics (air) as a falsework } & \begin{array}{l}\text { self-stabilizing abilities without } \\ \text { the use a binding agent. Simple } \\ \text { sis similary free-fliowing and can }\end{array}\end{array}$

be a complimentary system provide additional structural

the systems ability to compute
its own structura logic.
Maintaining this aspect is a

Maintaining this aspect is a
strong prionity and thus simple,

stow skil
valued.

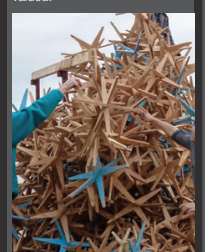

from the user/designer is still

required to achieve desired
distribution and coverage.

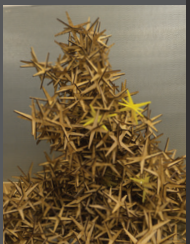
hybrid approach with ordered elements may serve as guid

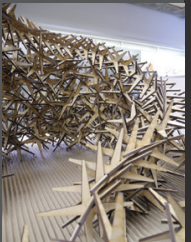

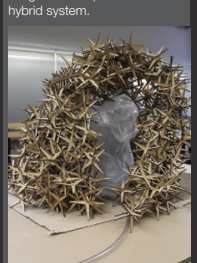

aggregate properties.

reversibility

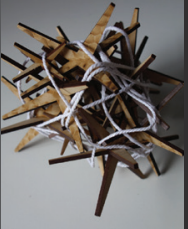

A rough surface is pited tor

fallure at the base. Embedding The system responds well to particles into the ground system excels in gravel or san

- carchboard sheet or rubber ma various forces. The system possess a form of sef healing forces can act as a pre-straining rat increases jamming

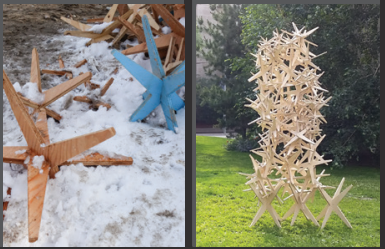
粼望 teve $x+4 x$
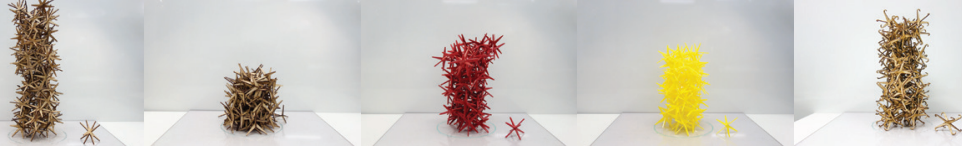

阷被 


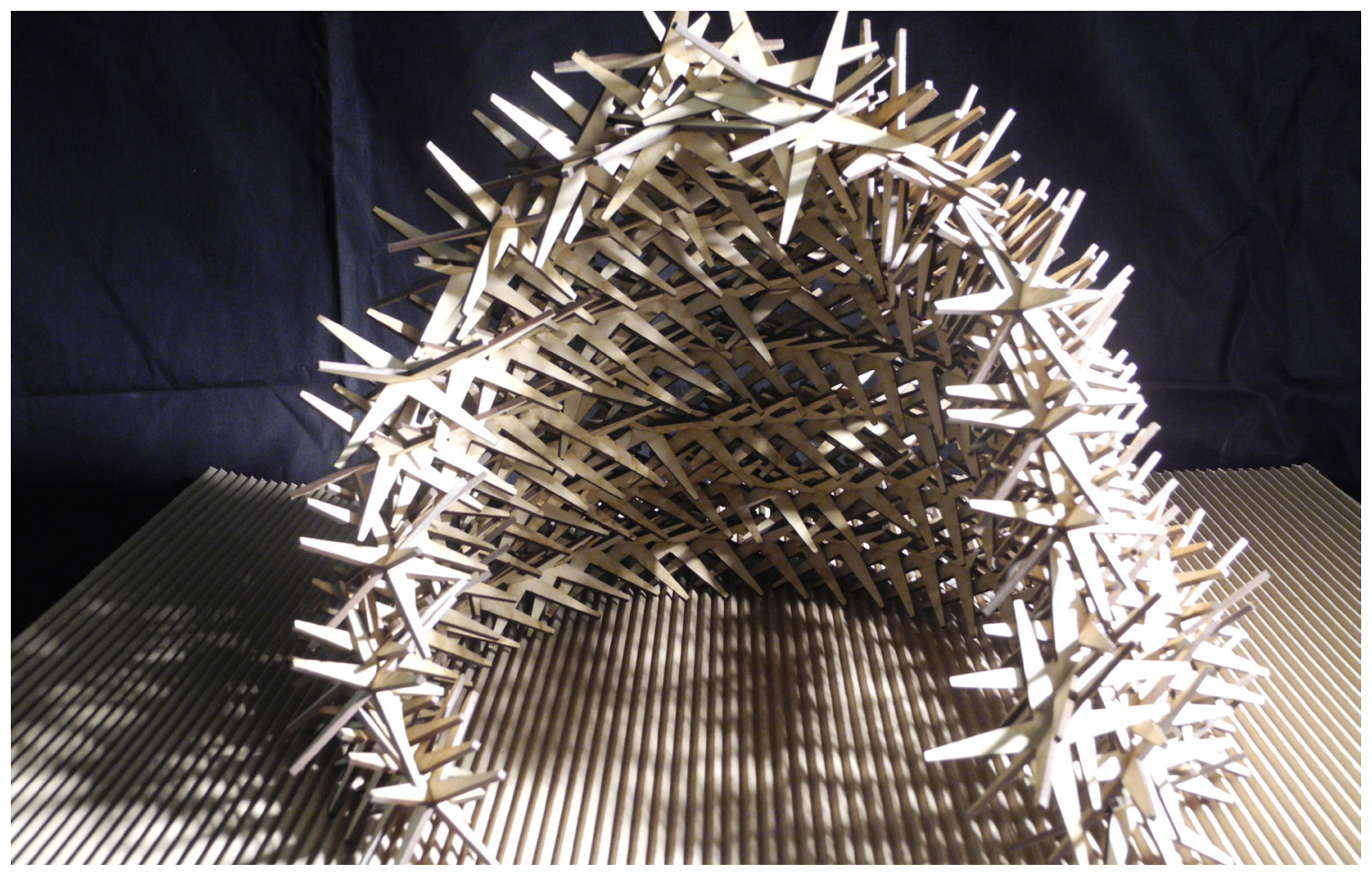

Ordered placement enclosure

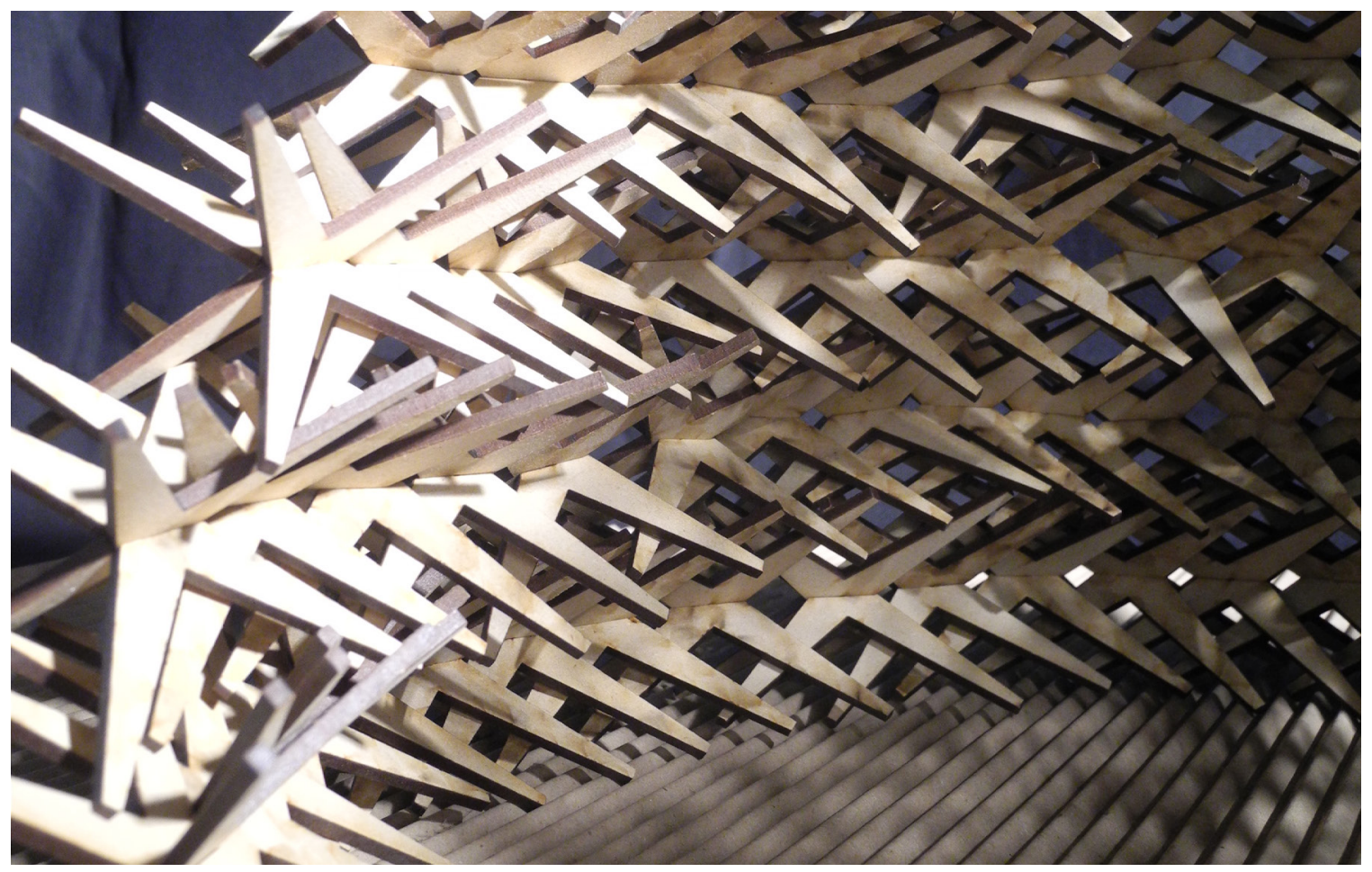

Close up of ordered placement enclosure 


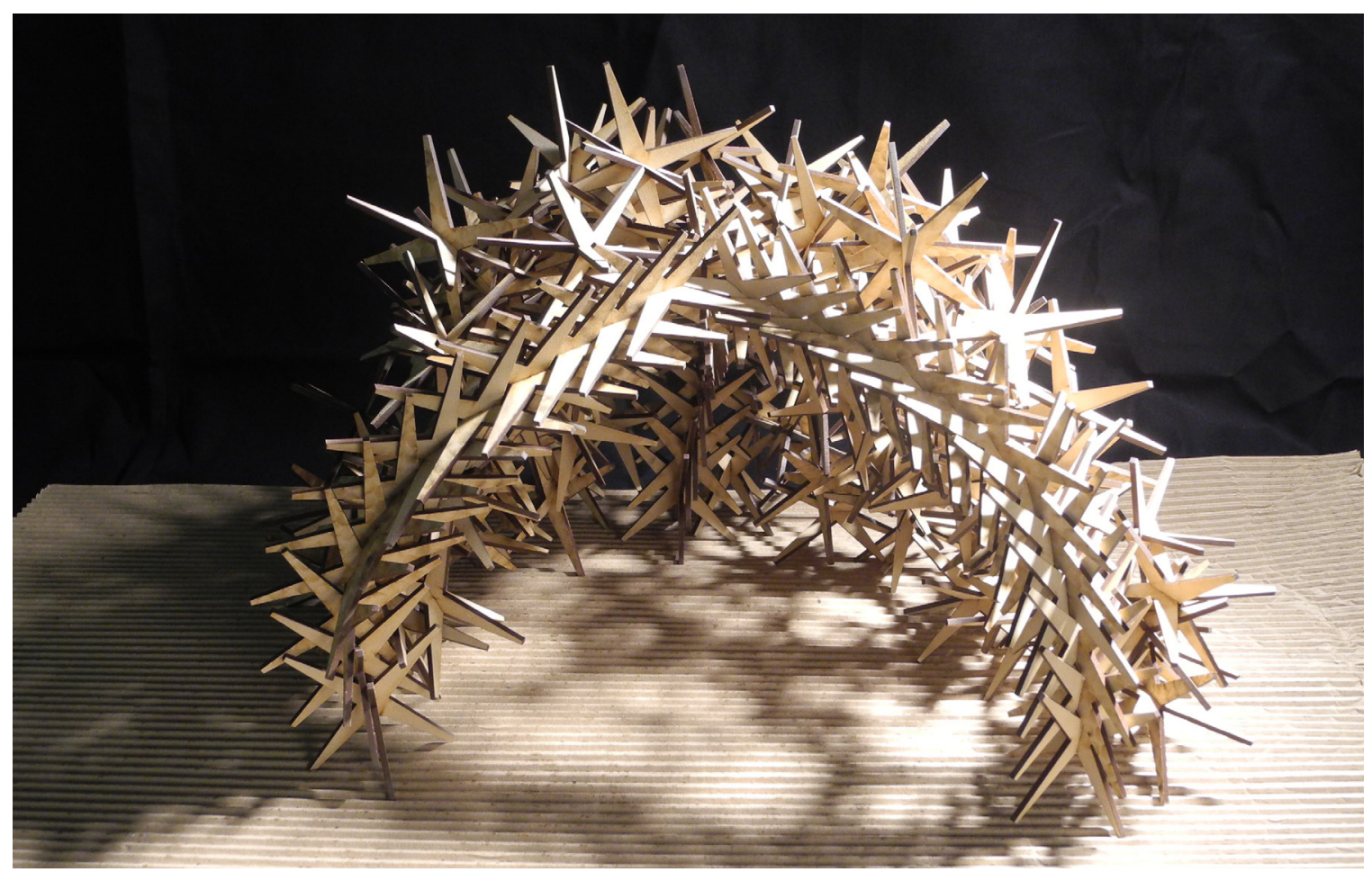

Hybrid ordered-self-organized vault enclosure

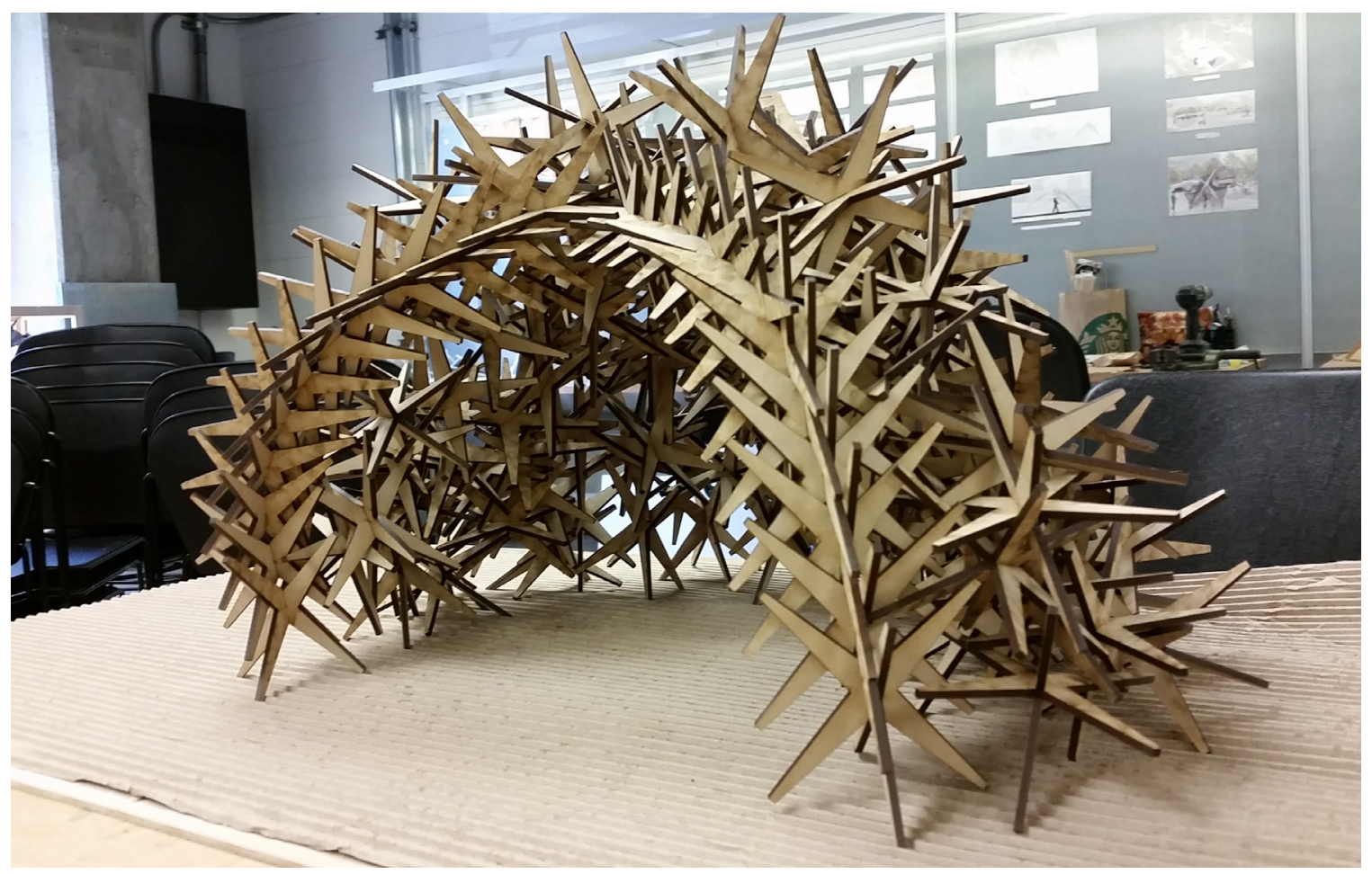

Hybrid approach could allow for controlled openings 


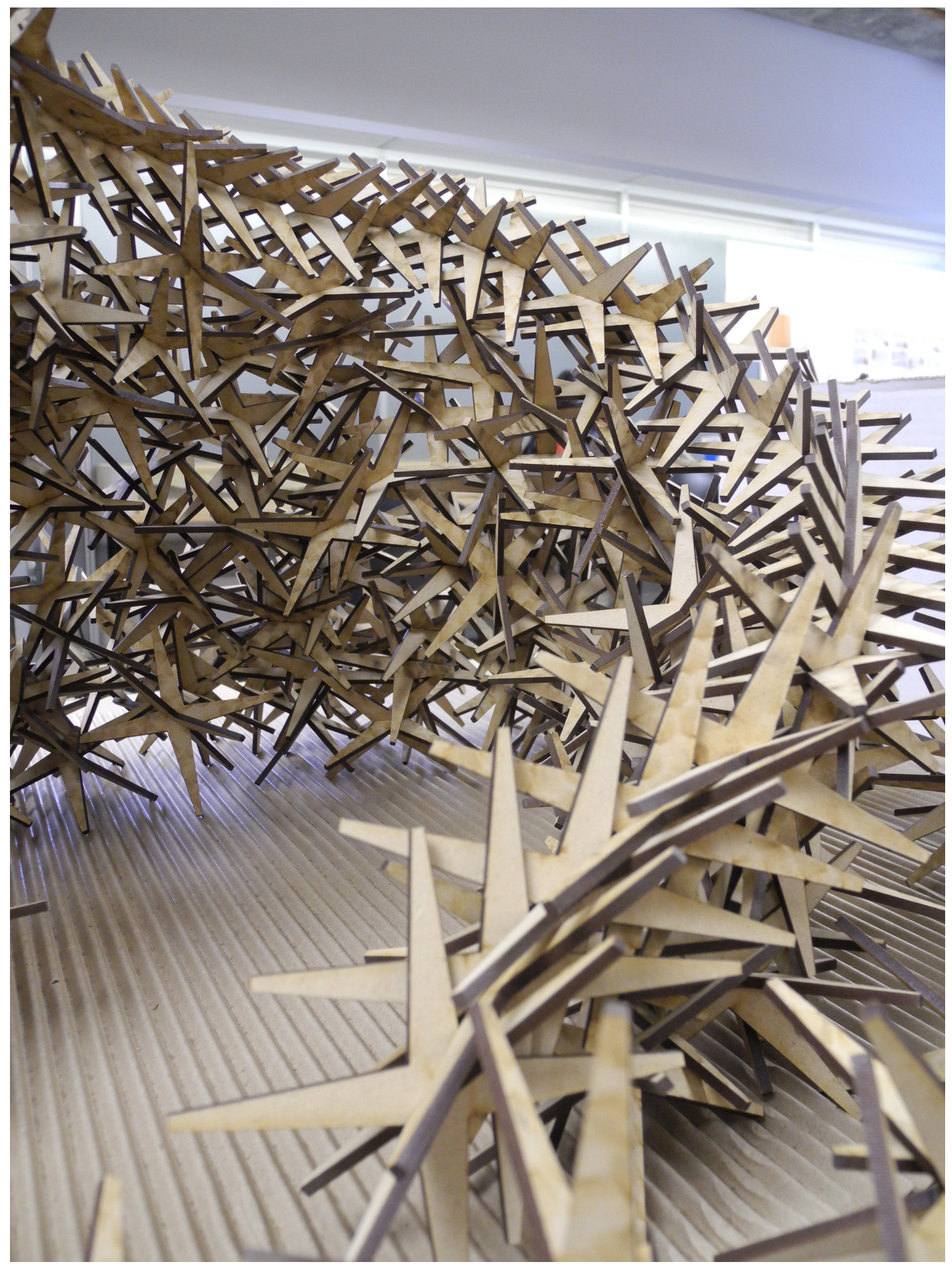

Development using a combination of ordered and self-organizing processes

182 Architecture at the Edge of Chaos 


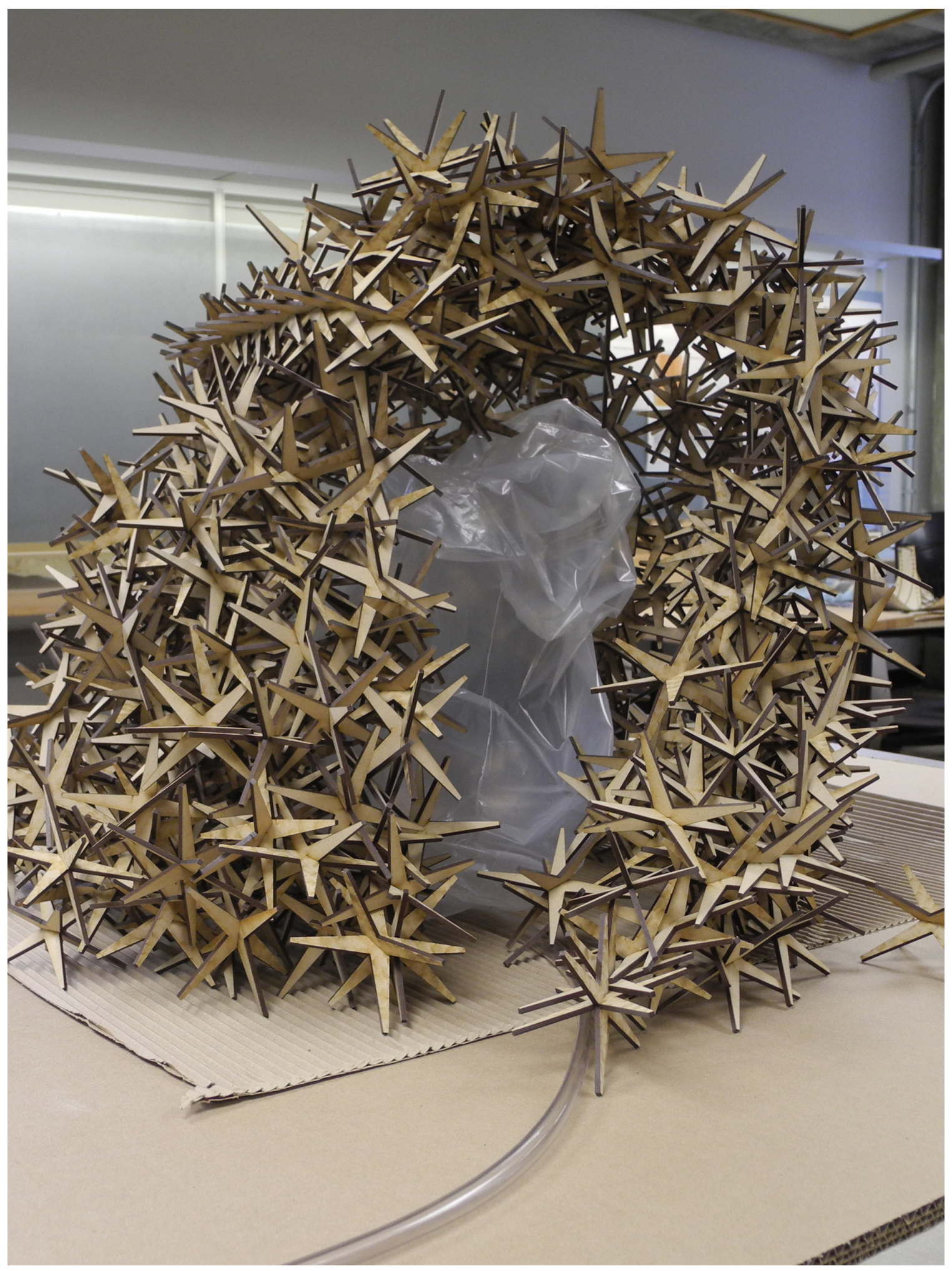




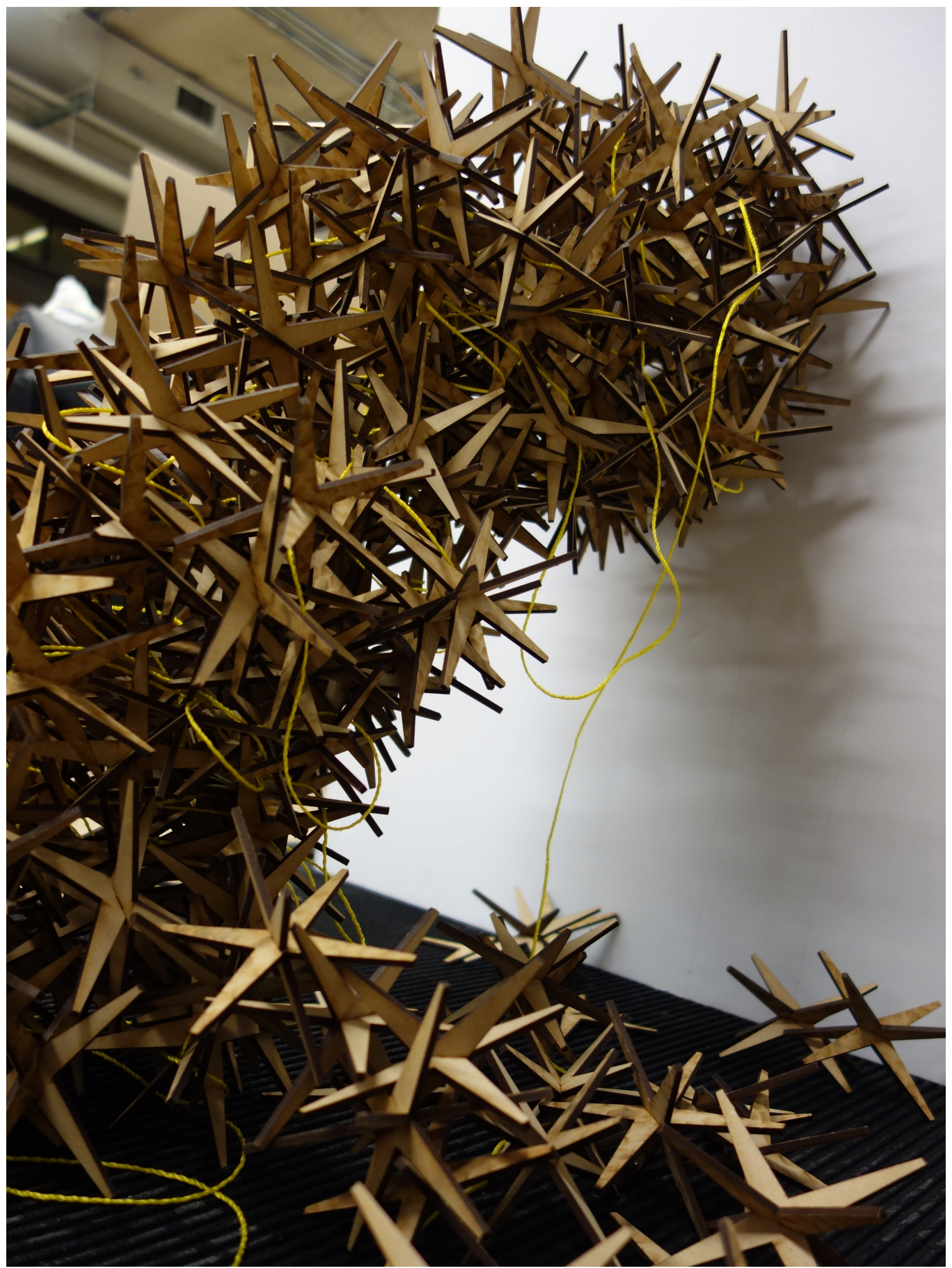

Arched cylindrical form with intermediate string layers 


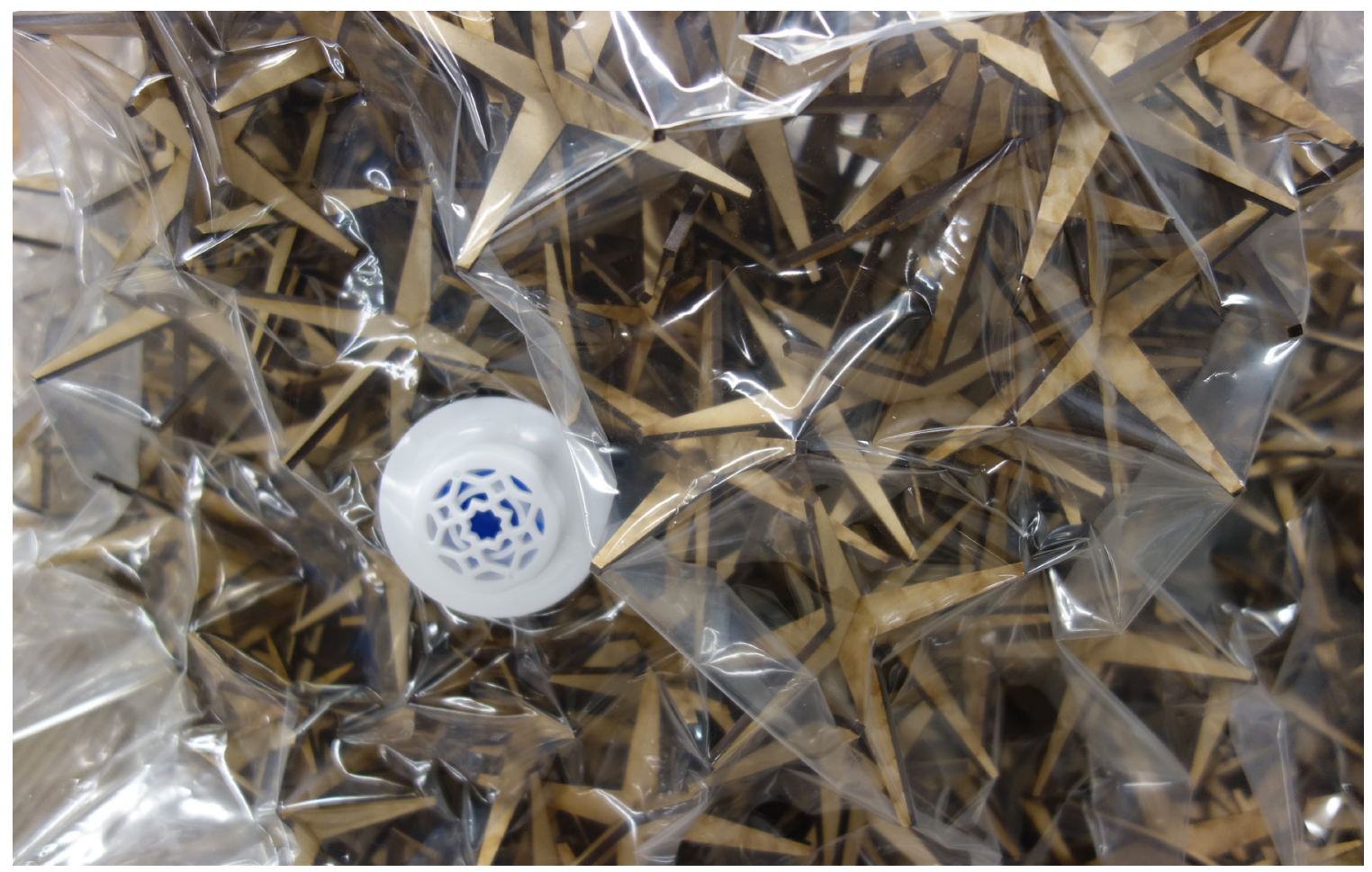

Vacuum jamming as a complementary process/system

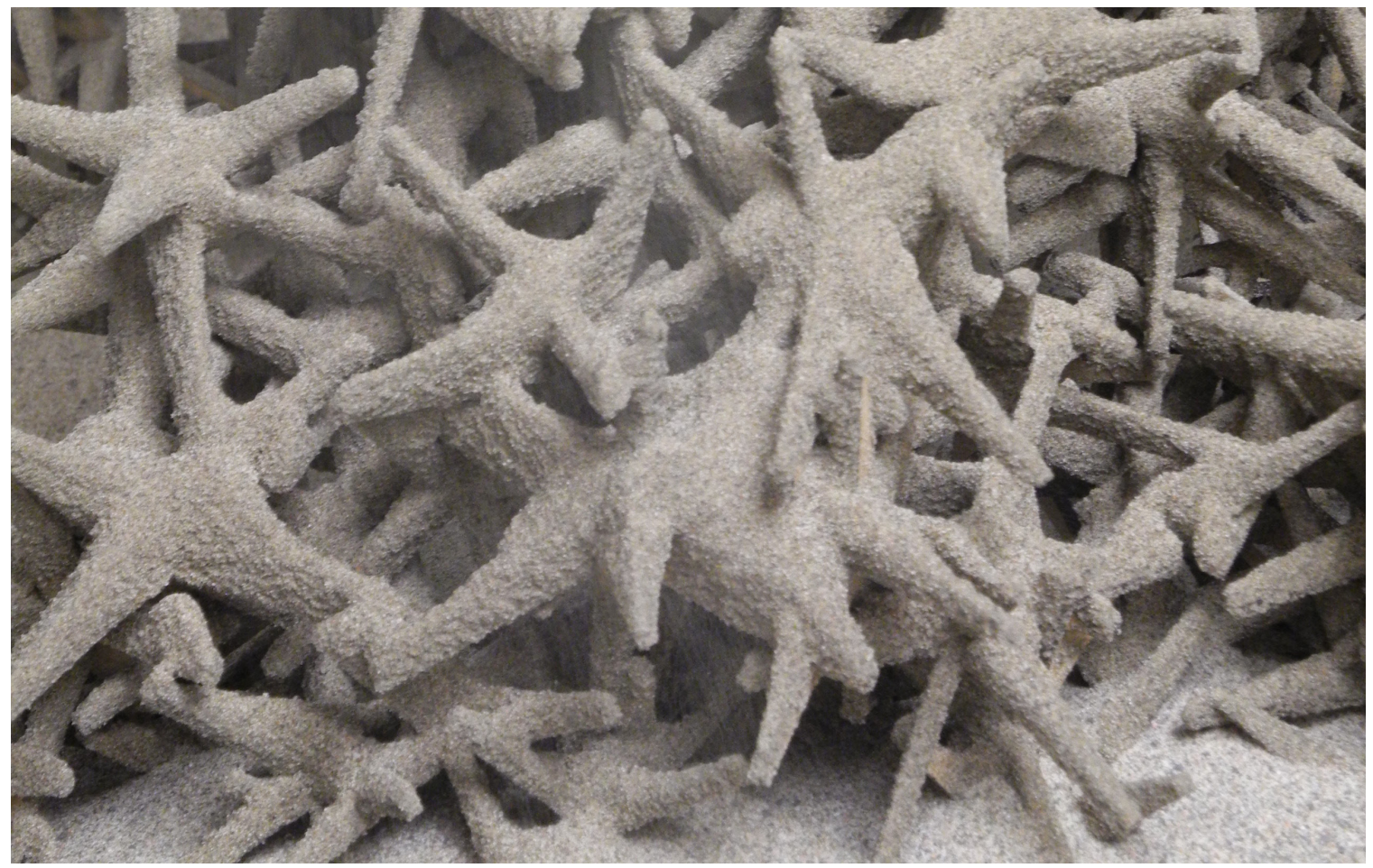

Accumulation of natural granular material (sand) on structure 
Appendix B: Sands of Tomorrow (Sukkahville 2015)

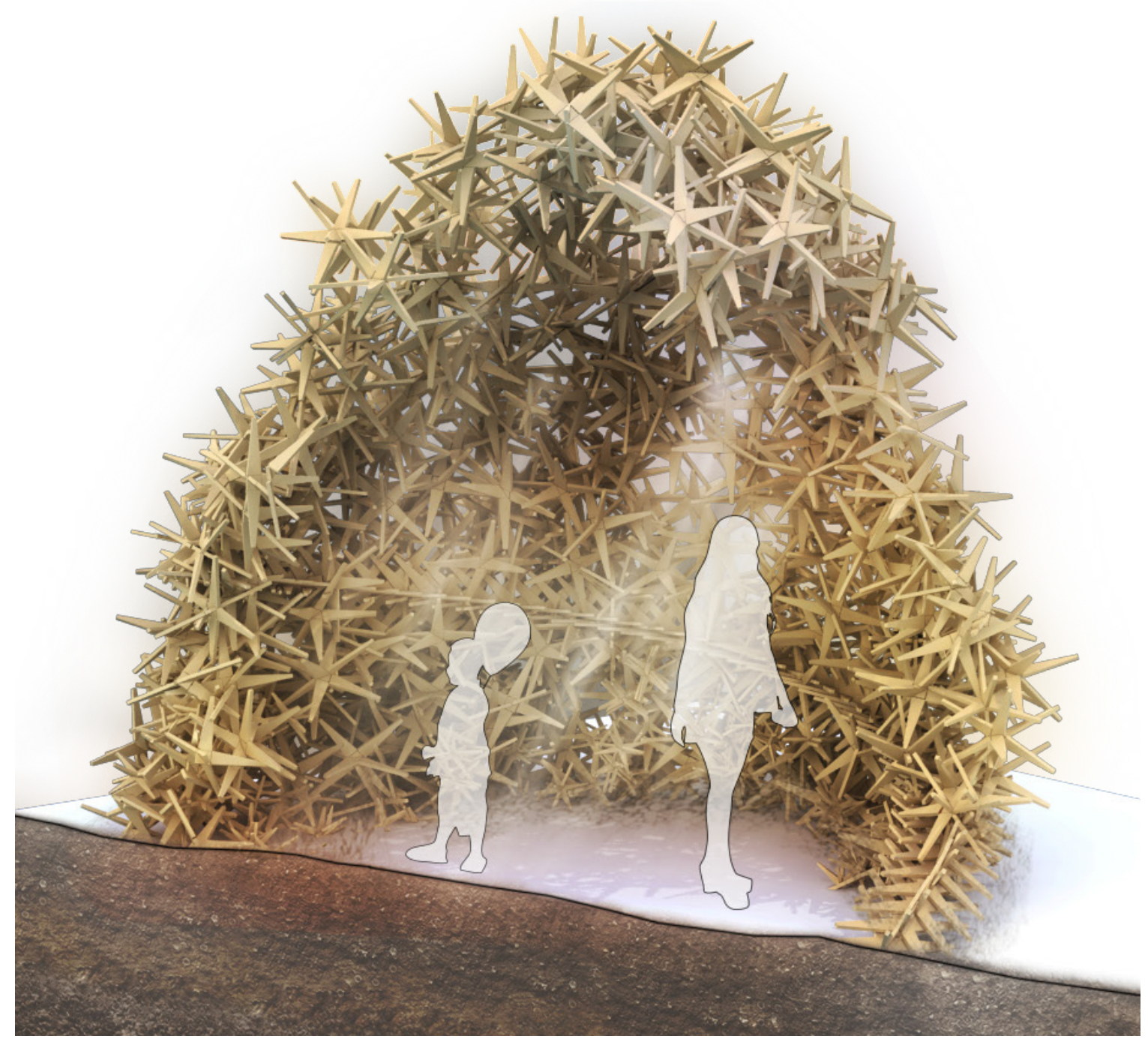

Enclosure concept

186 Architecture at the Edge of Chaos 

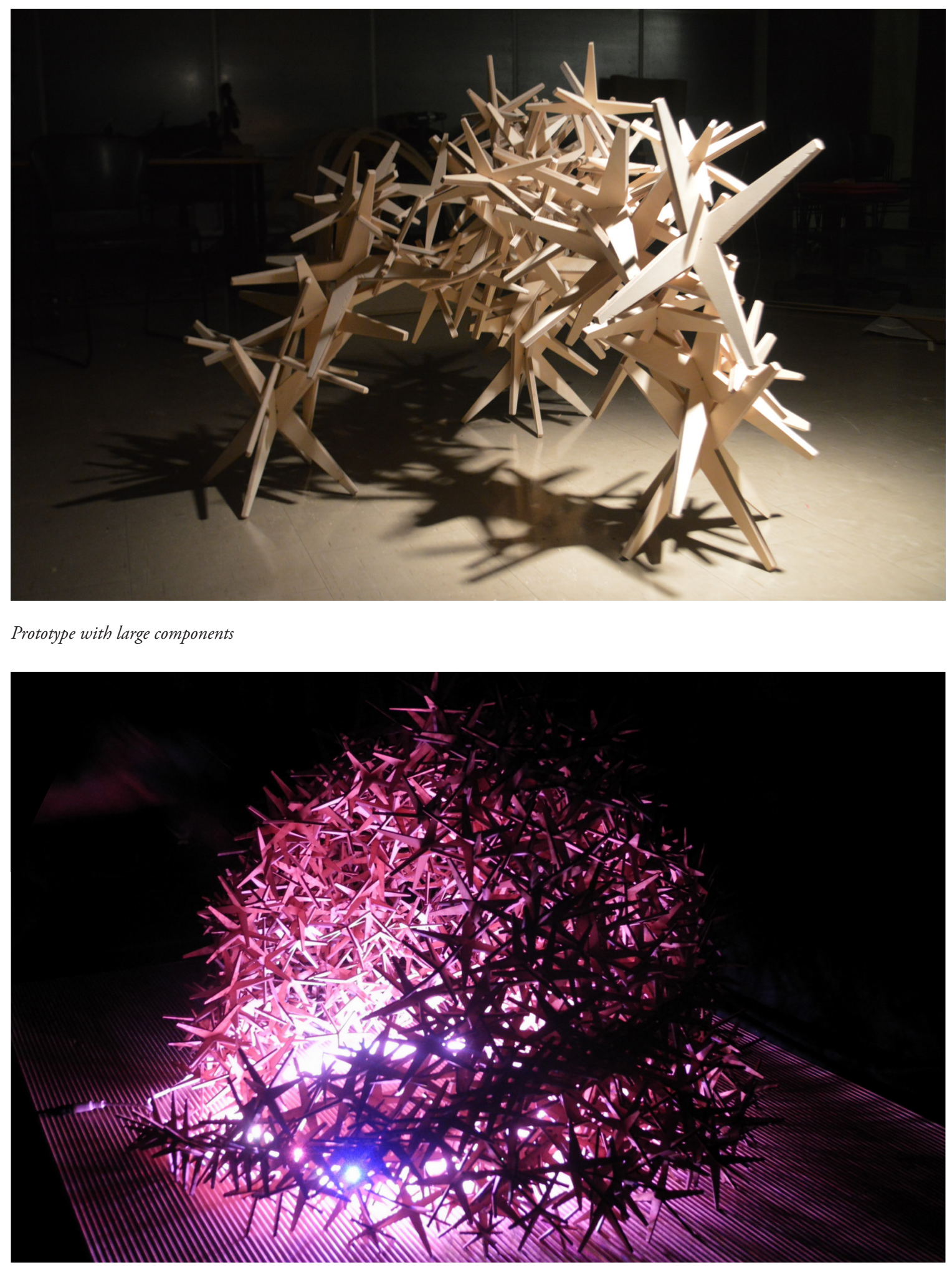

Lighting from within 


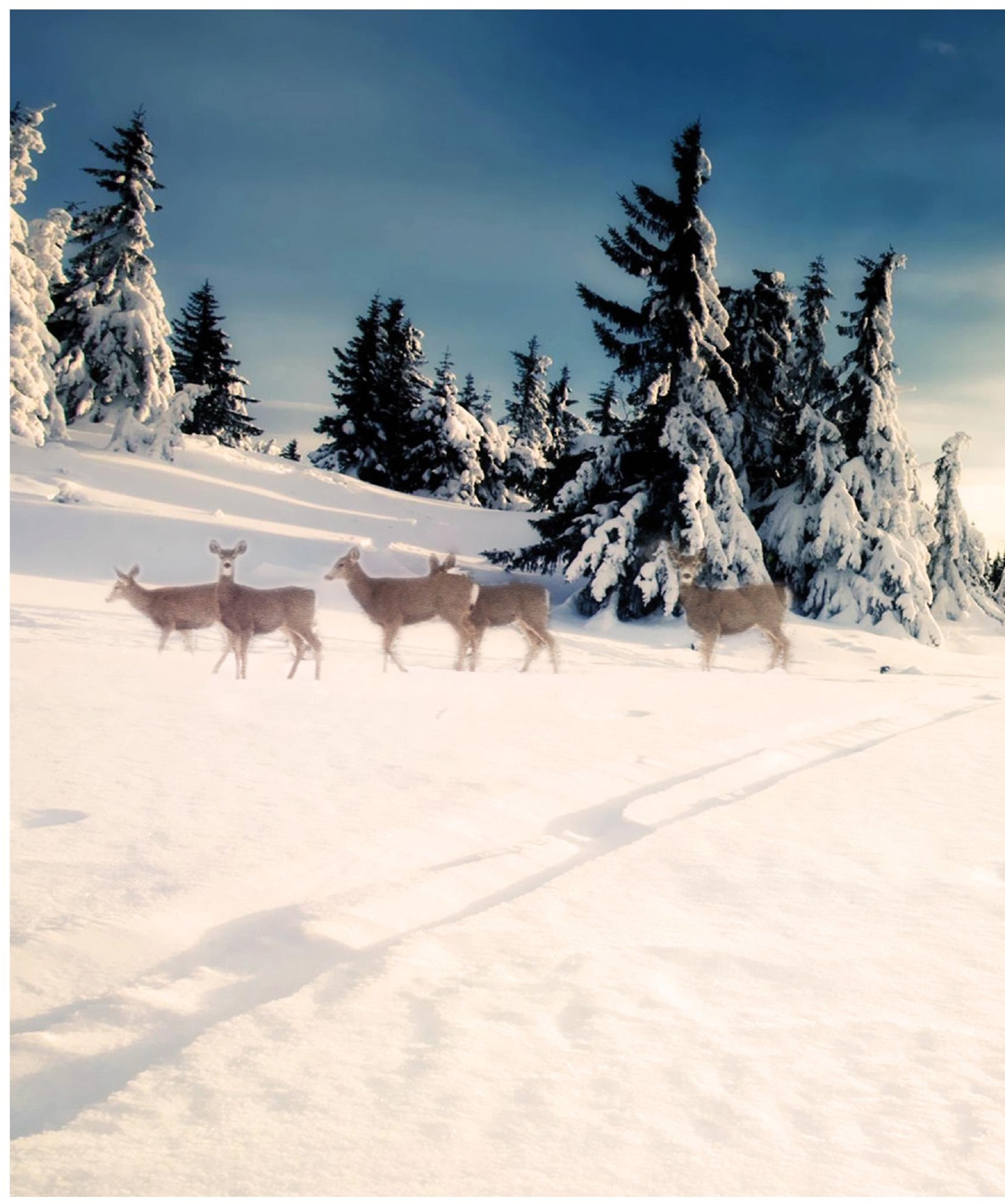

The system has strong connections with the landscape

188 Architecture at the Edge of Chaos 

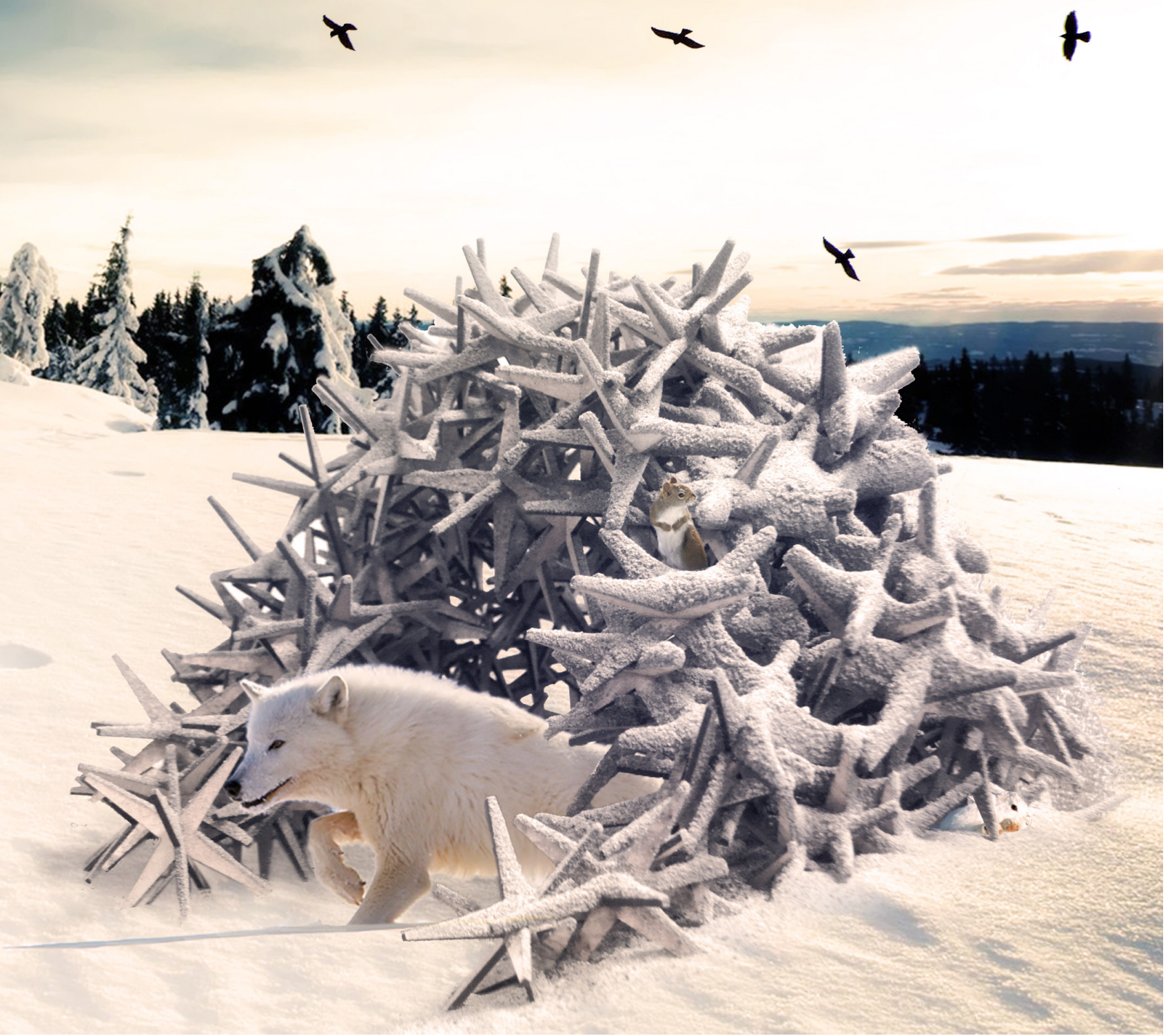
Appendix C: Flow (Winter Stations 2016)

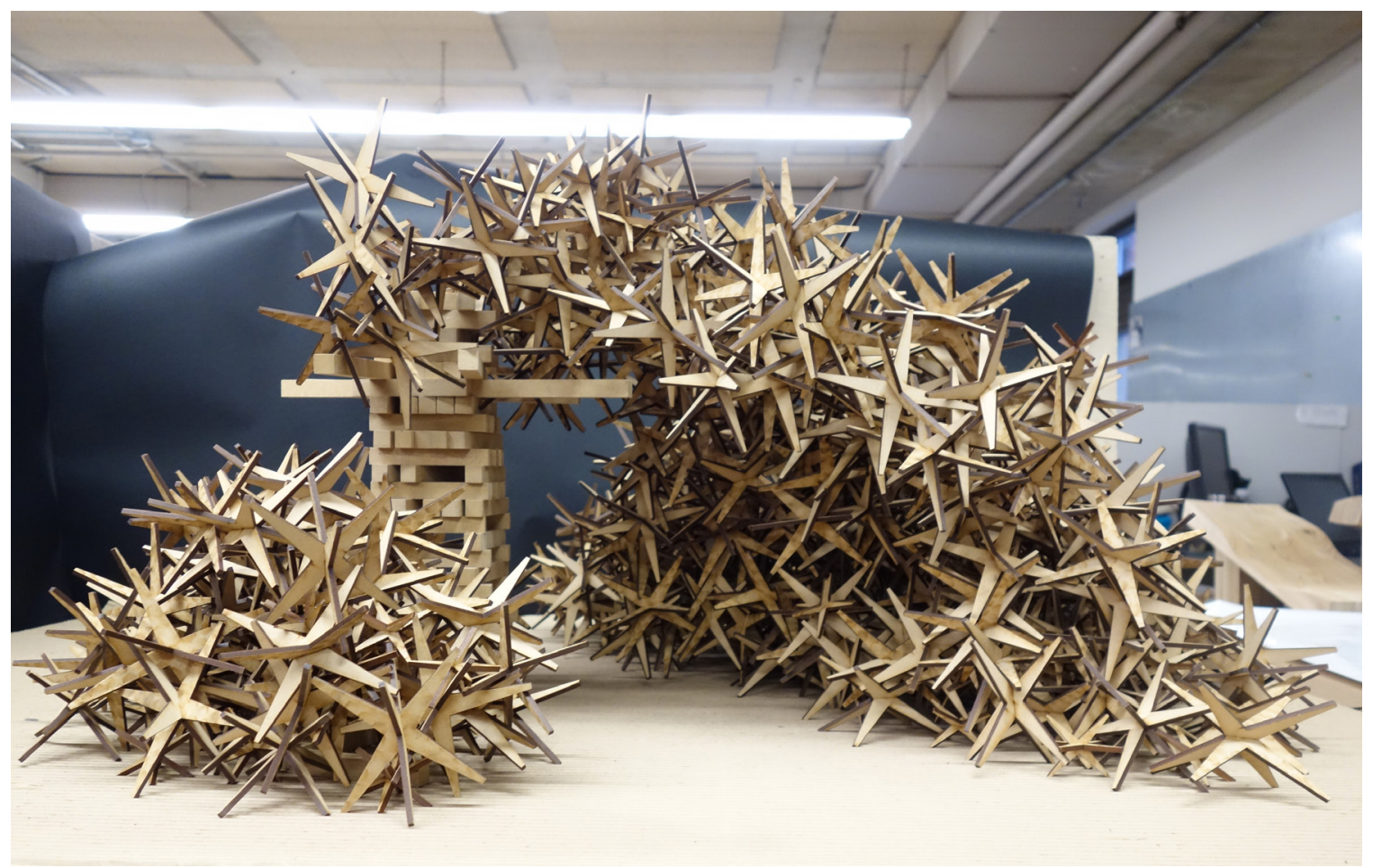

Scale model 


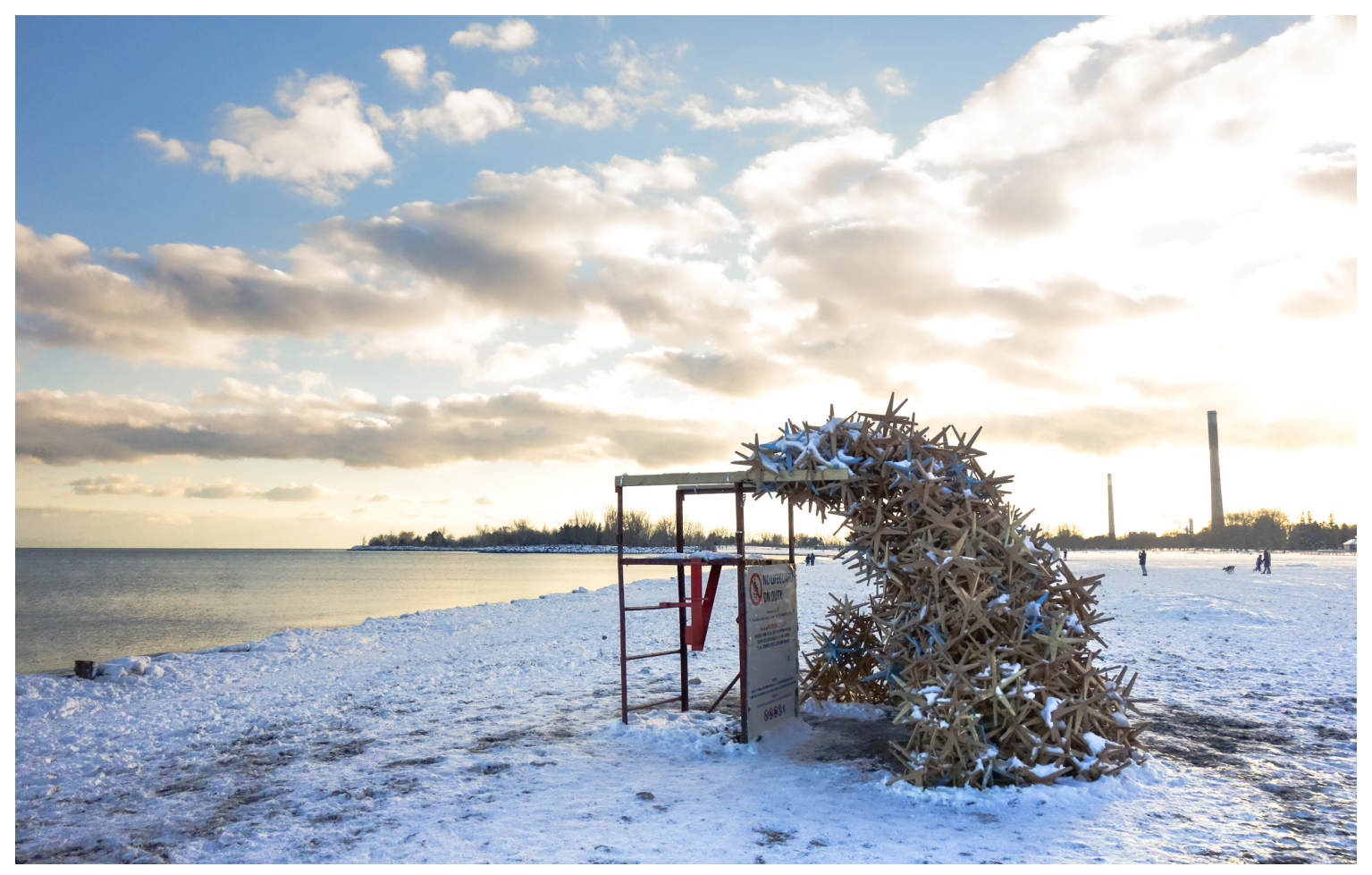

Realized construction at the beach

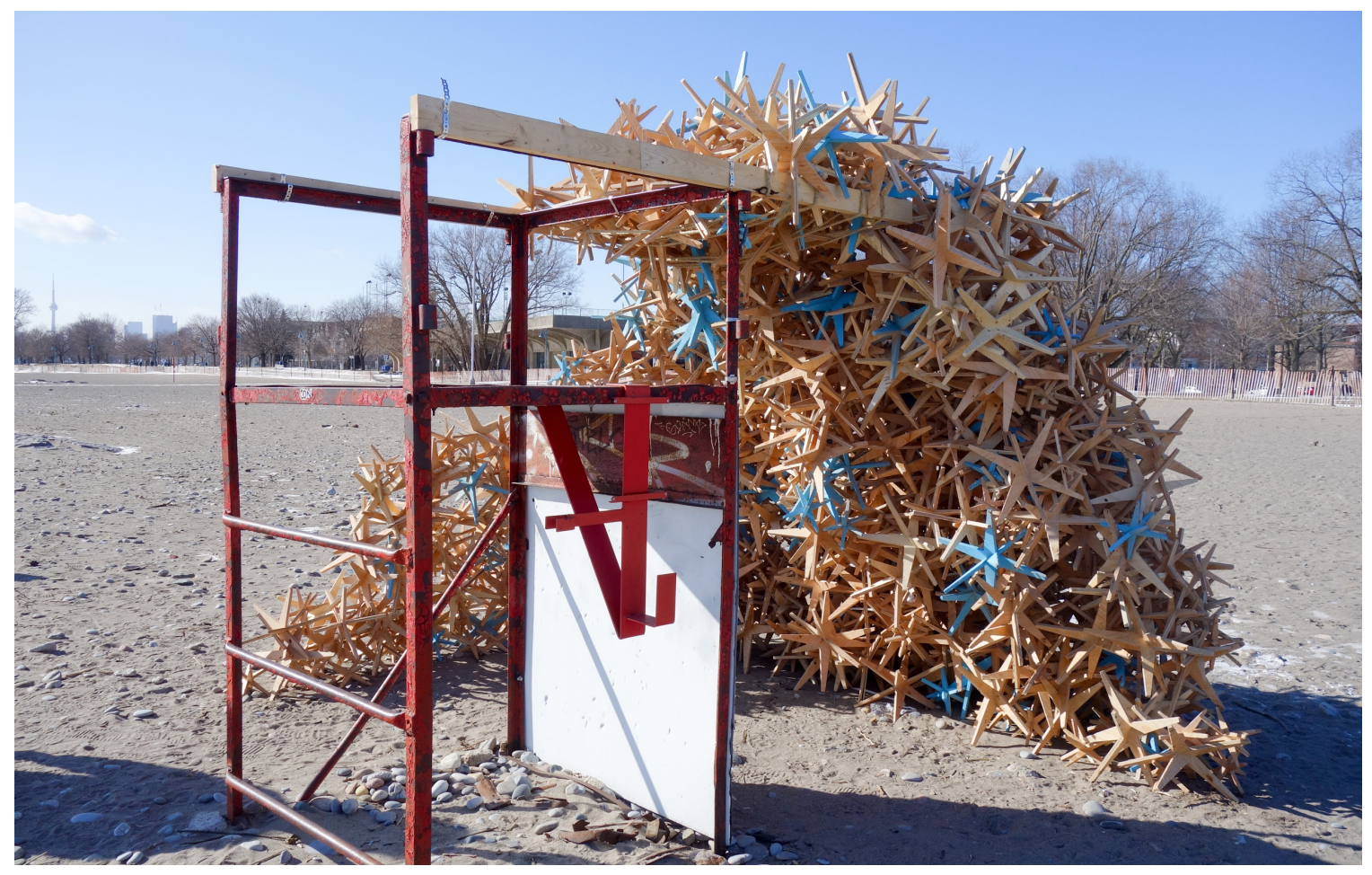

A light rope net is used for overhead structure 


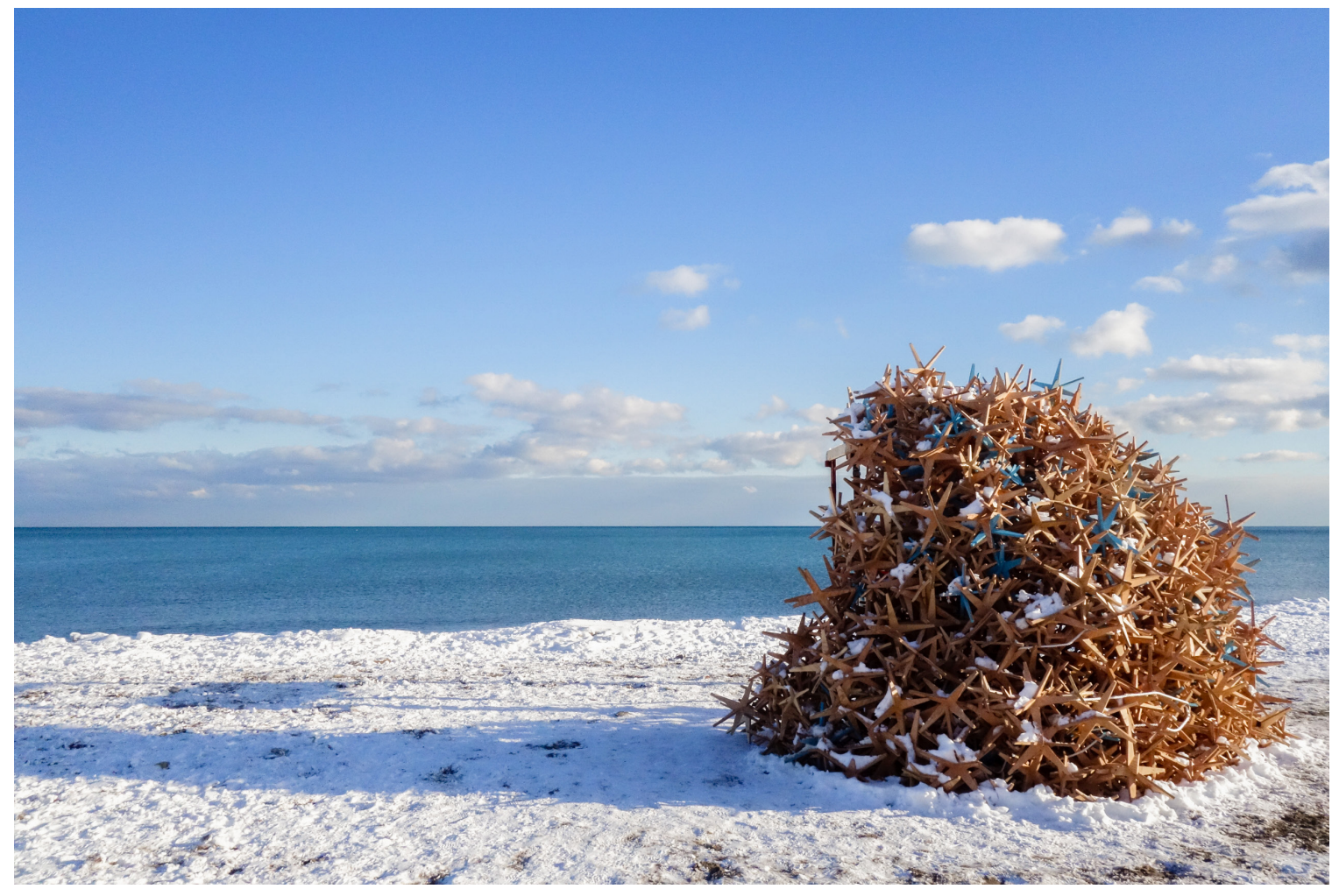

View from the rear with lake context

192 Architecture at the Edge of Chaos 


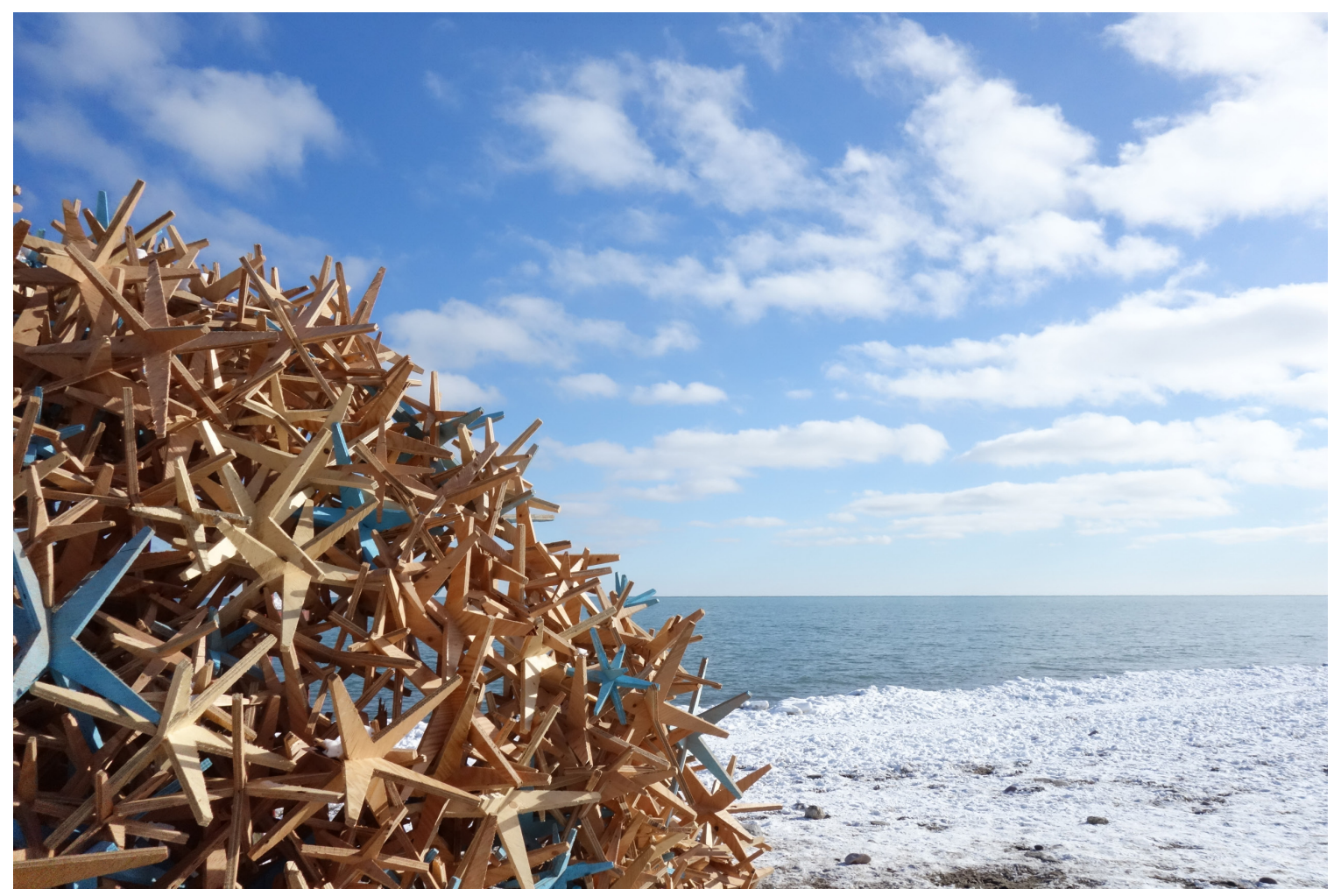

Its permeable structure allows air and light to pass 


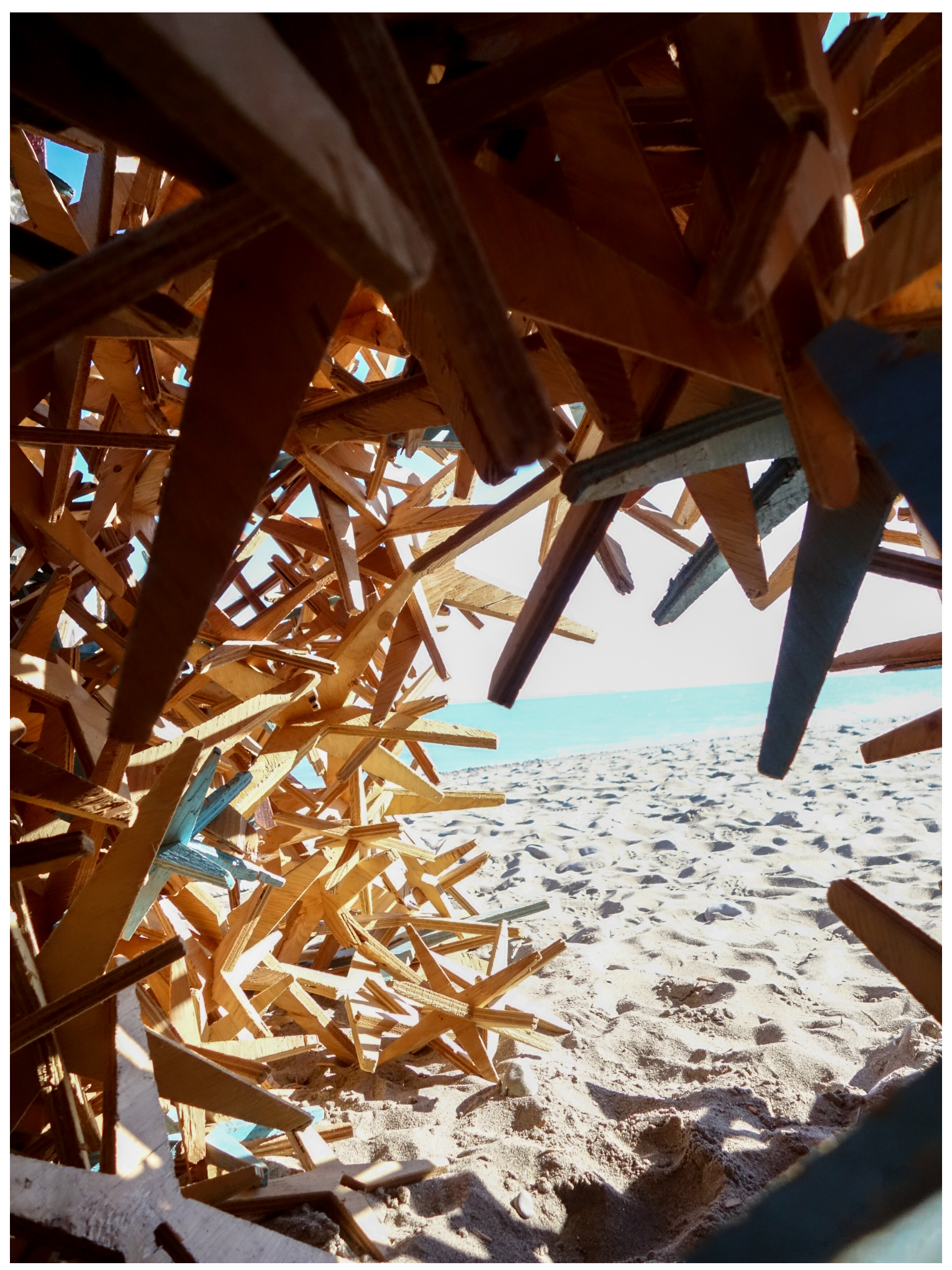

View from self-formed cavern-like space 


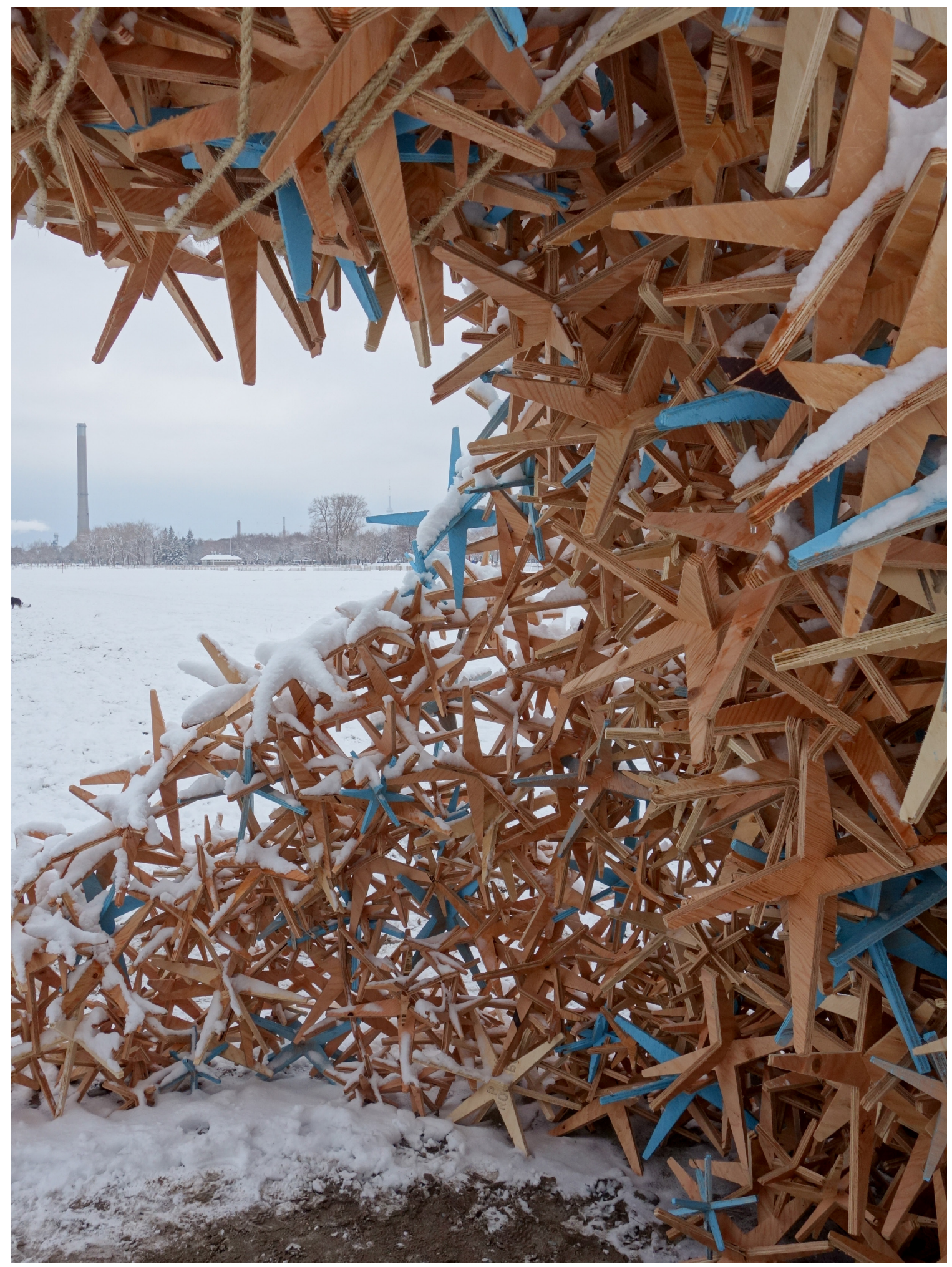

View from under the arch 


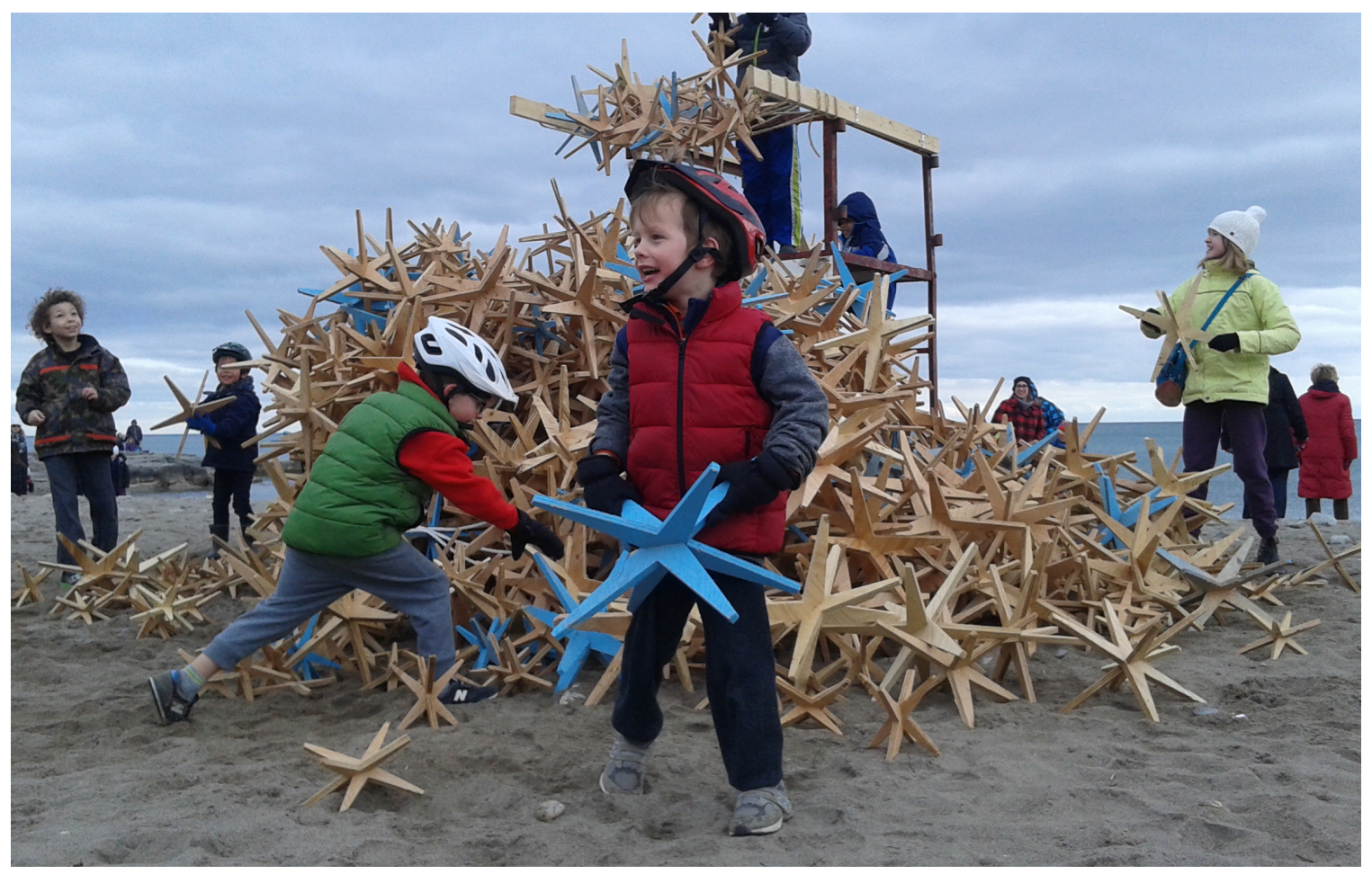

Innate playful quality promotes participation. By Christine Chung

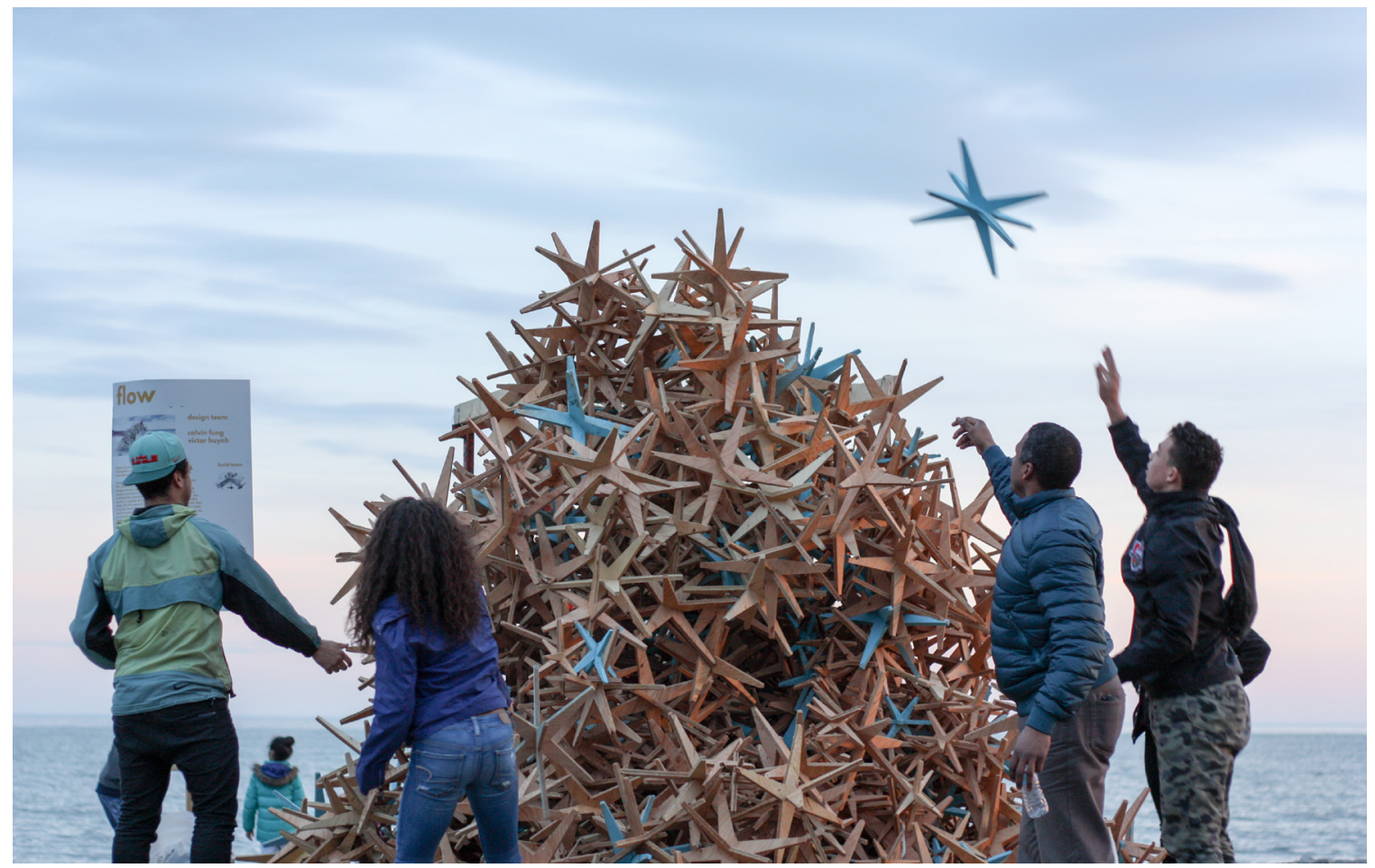

Simplicity engages visitors of all ages 


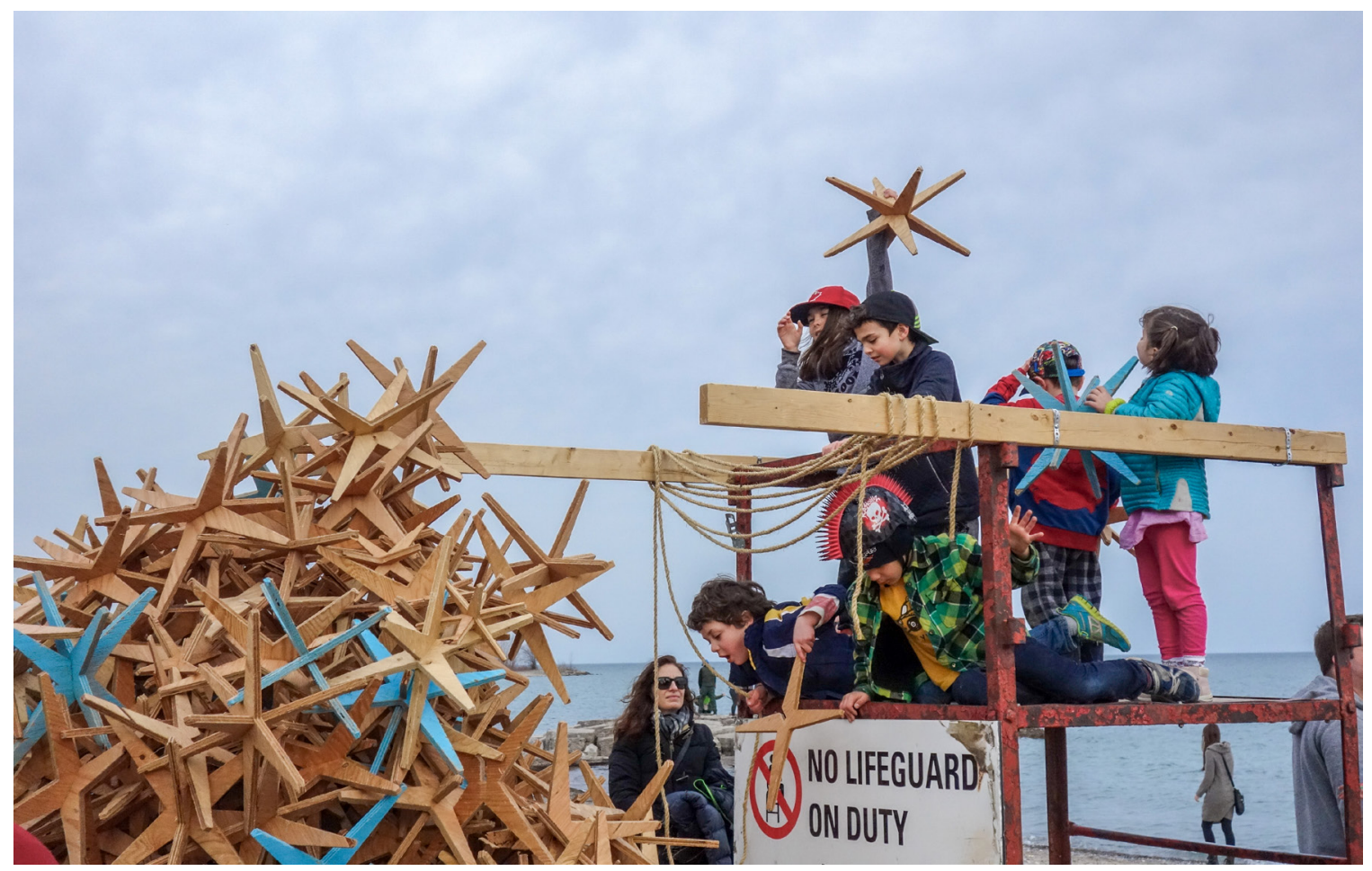

Forms are created by roughly tossing elements together or carefully sculpted.

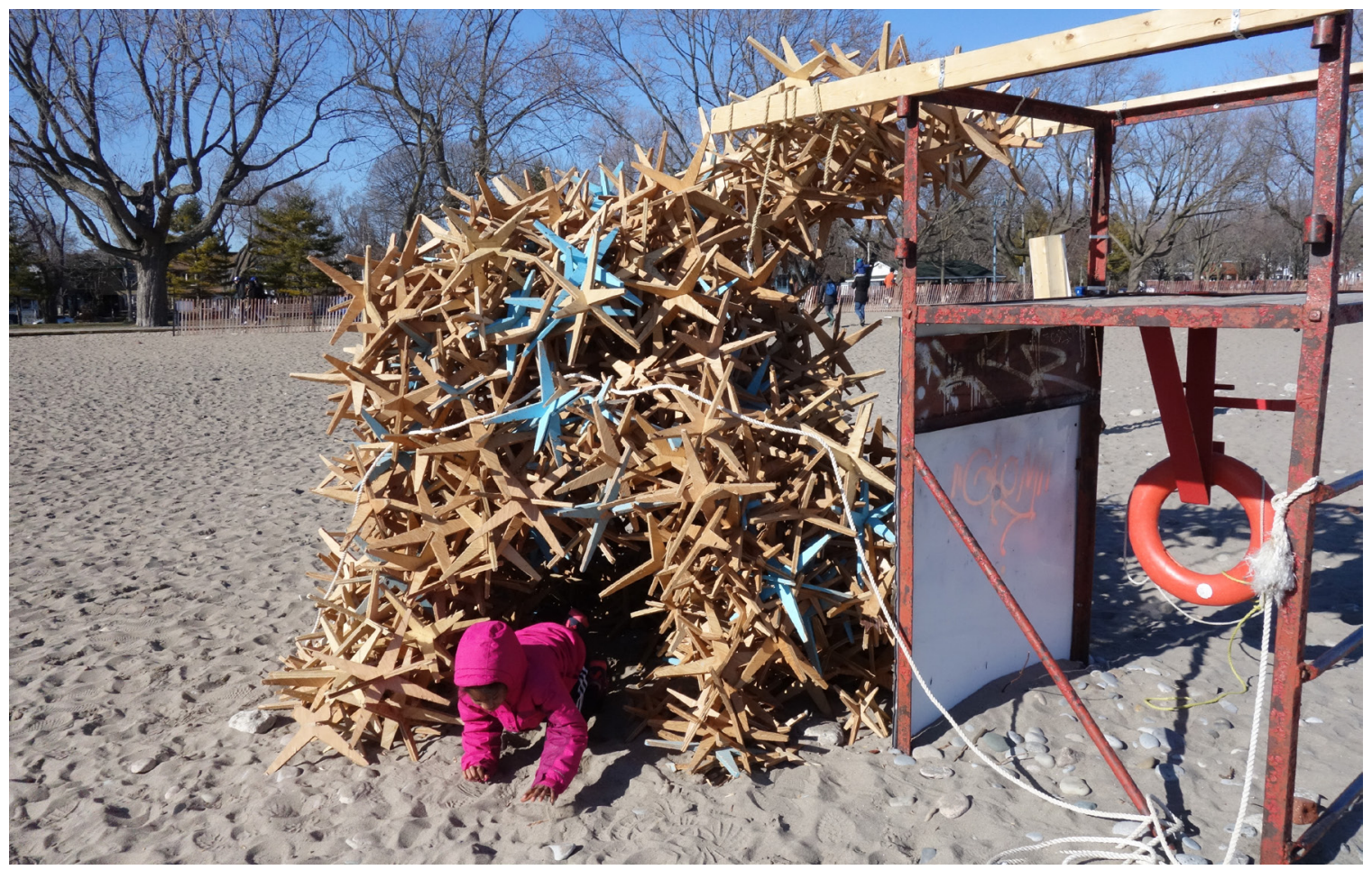

A primitive hut was naturally form 


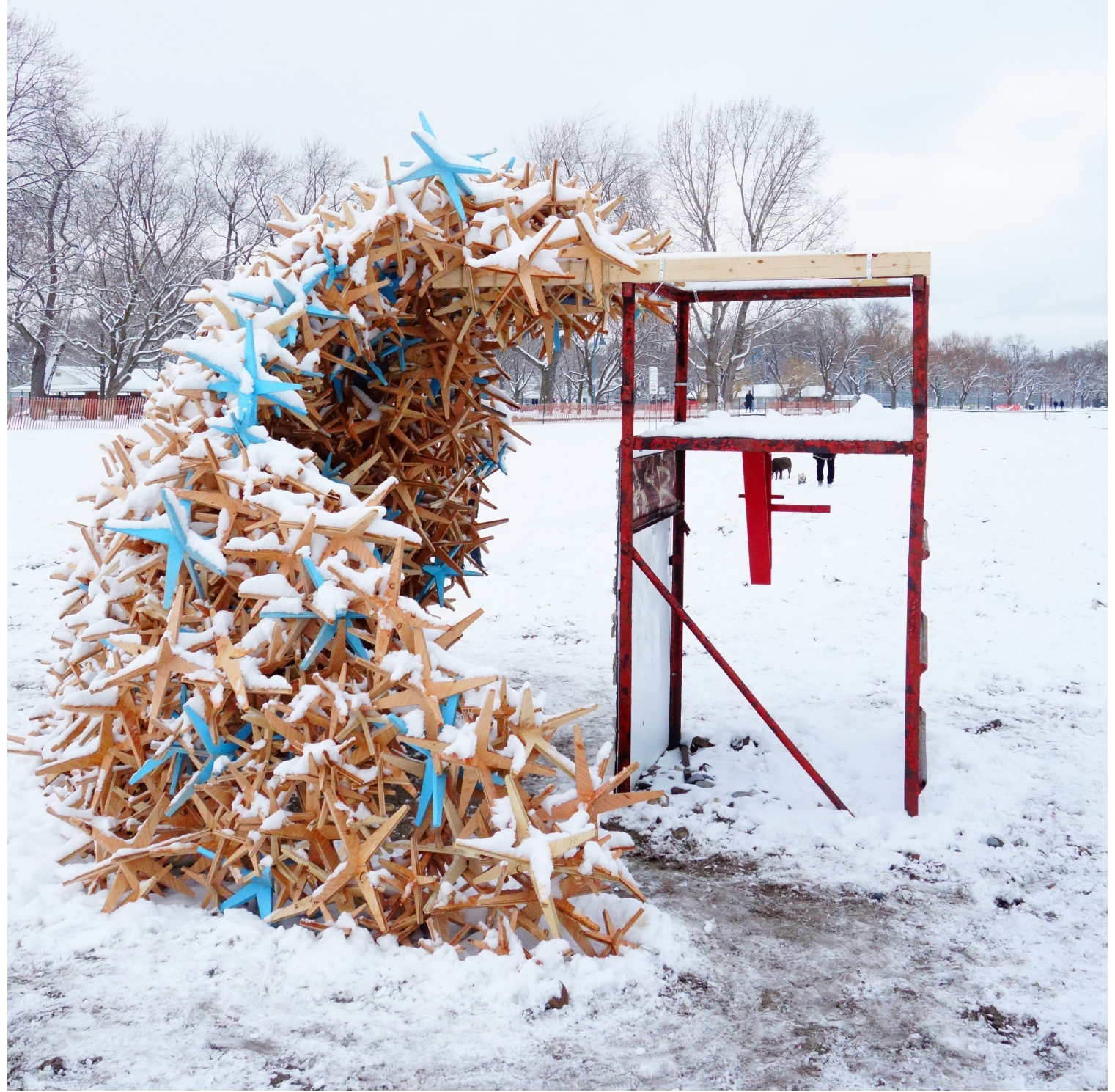

Initial form

198 Architecture at the Edge of Chaos 


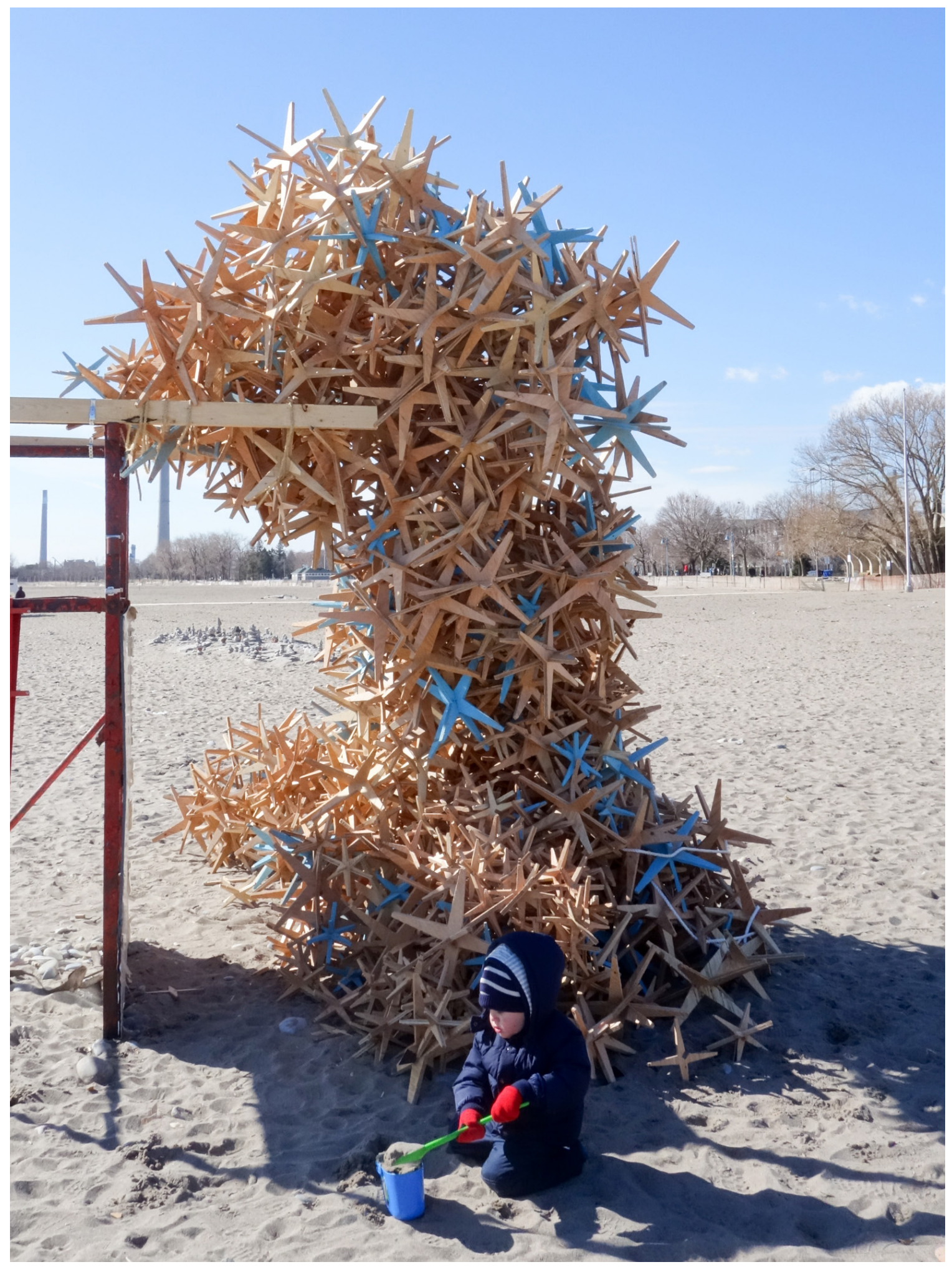

Evolving form 
Appendix D: Seeds (Allan Gardens)

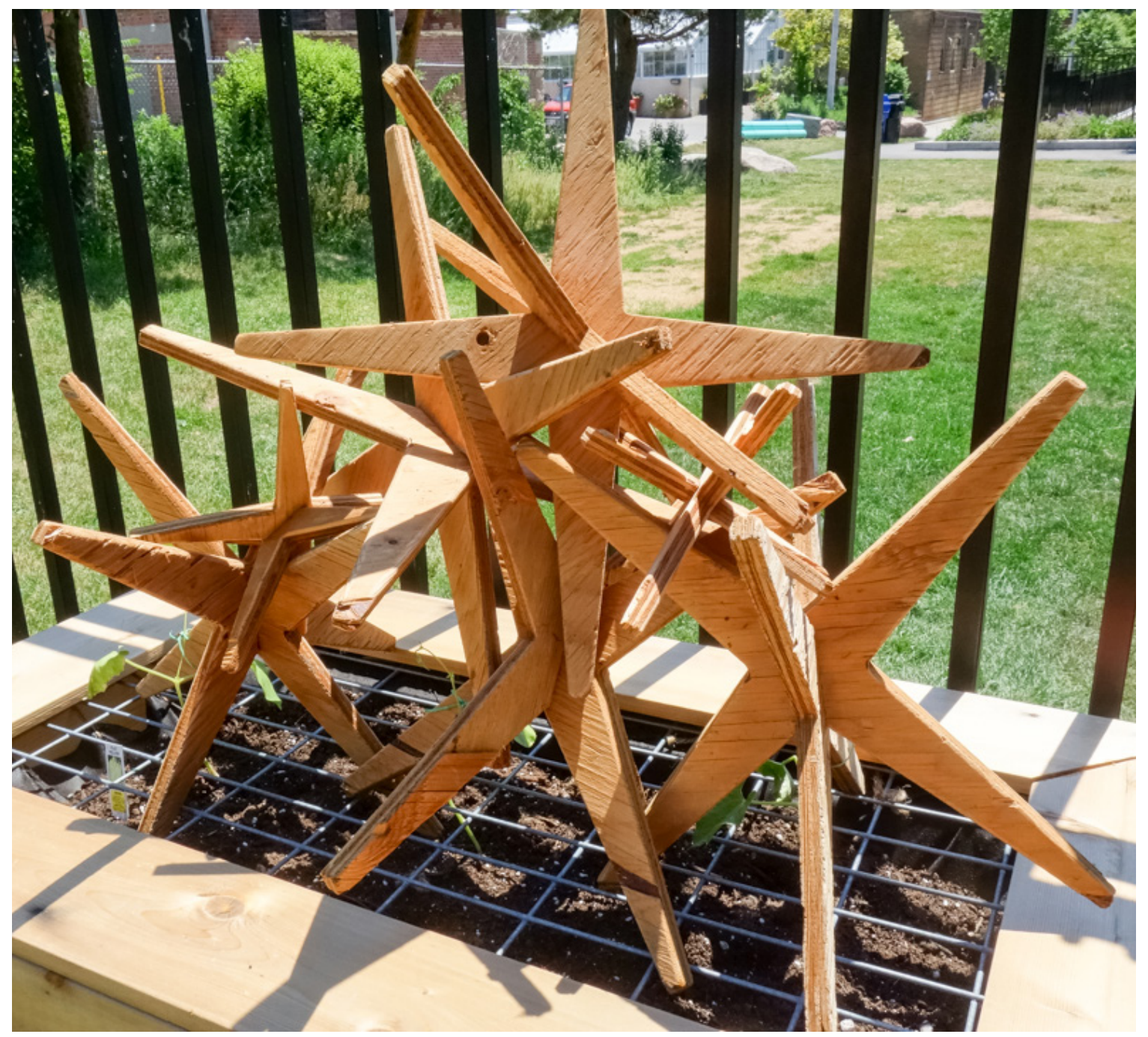

Armature for plant growth 


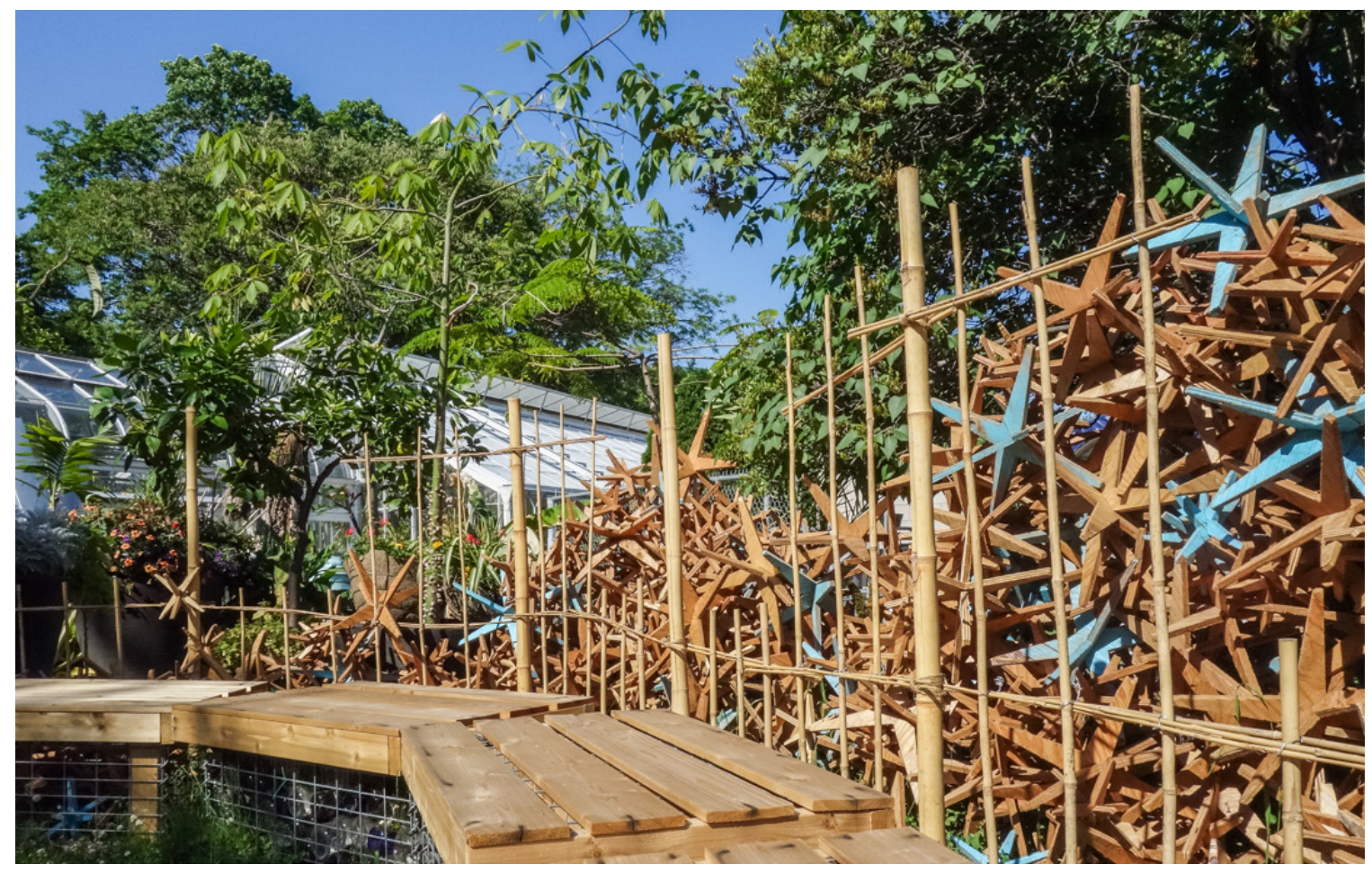

Screen feature

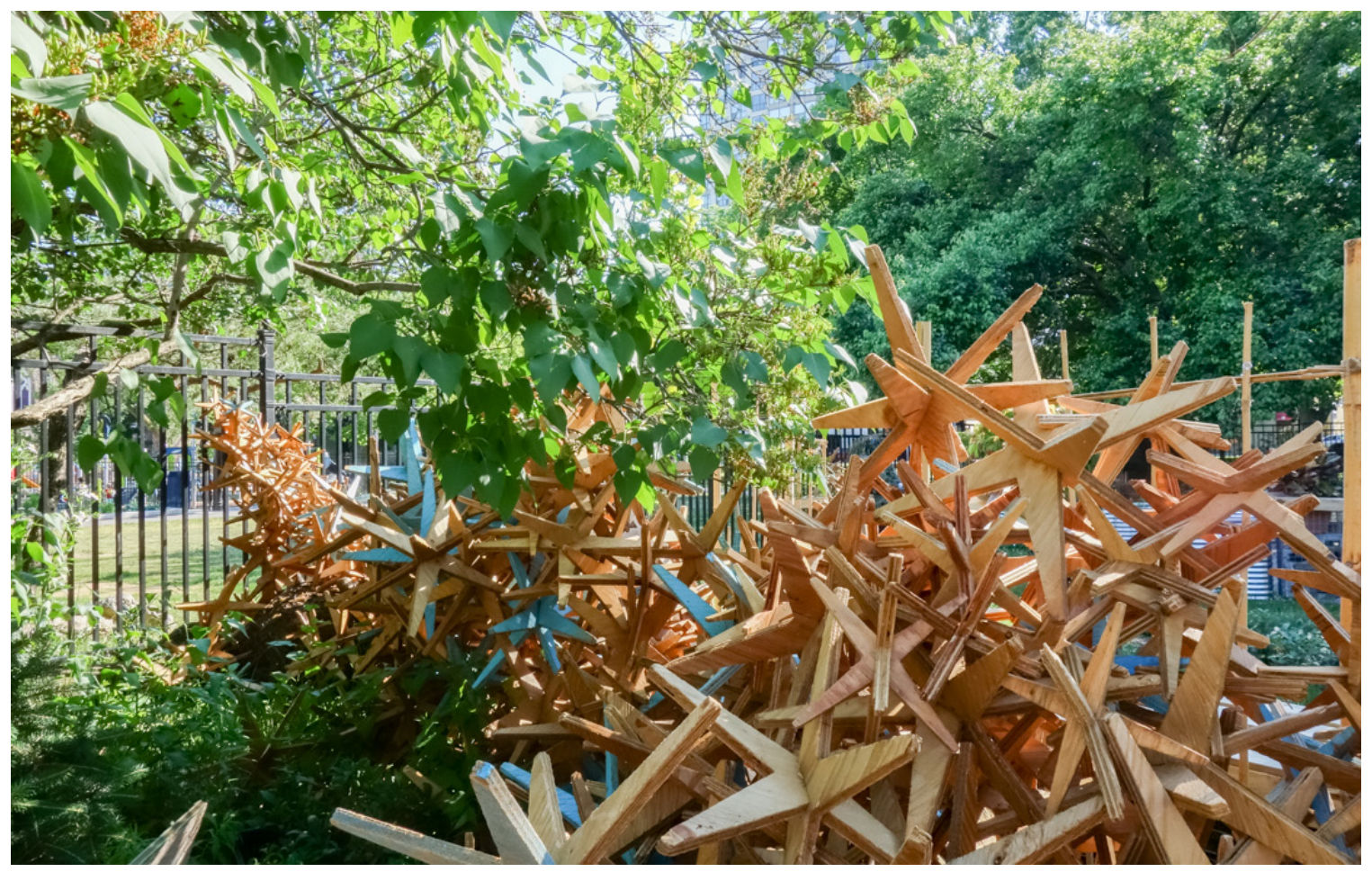

Wood material compliments the its natural surroundings 


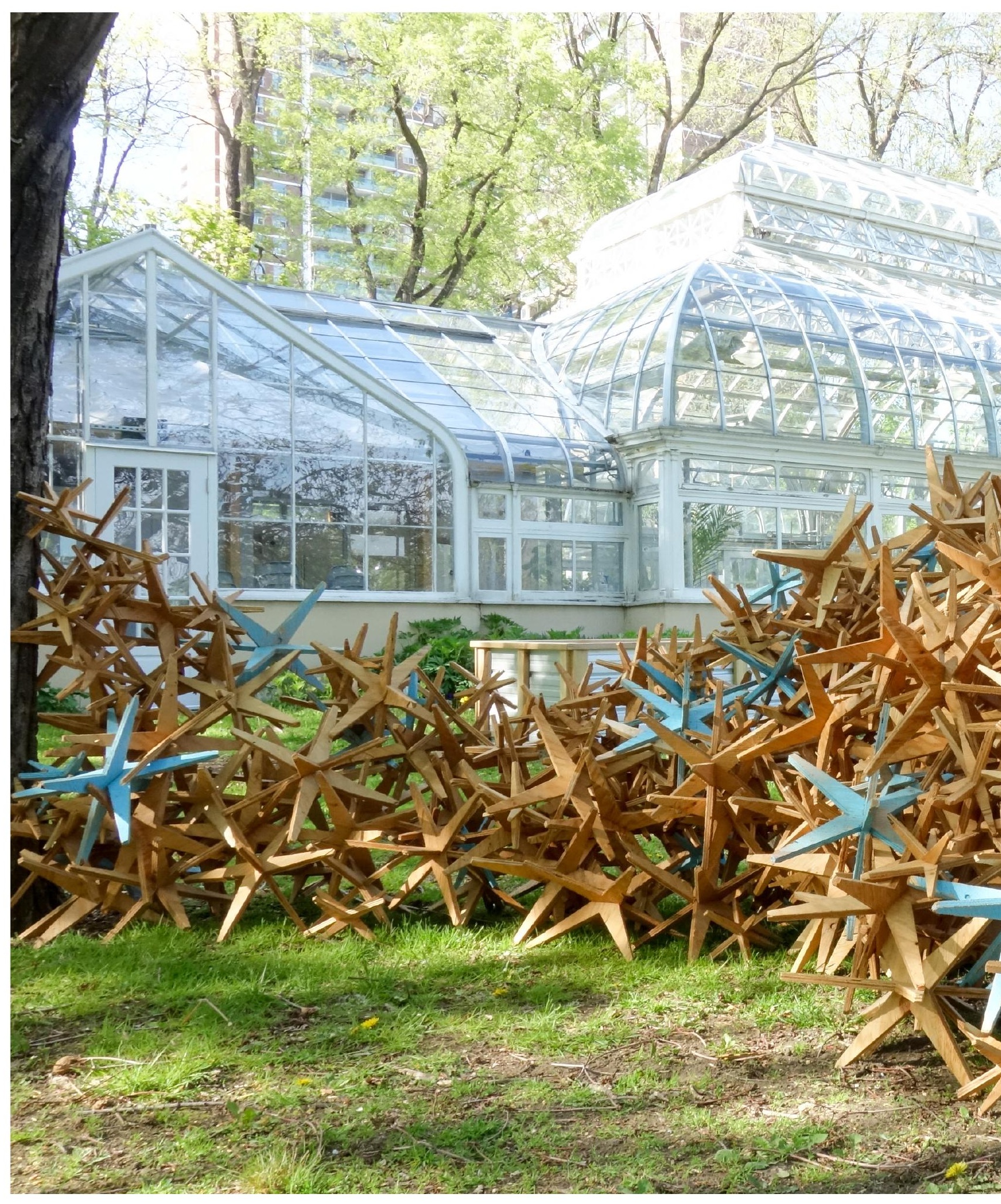




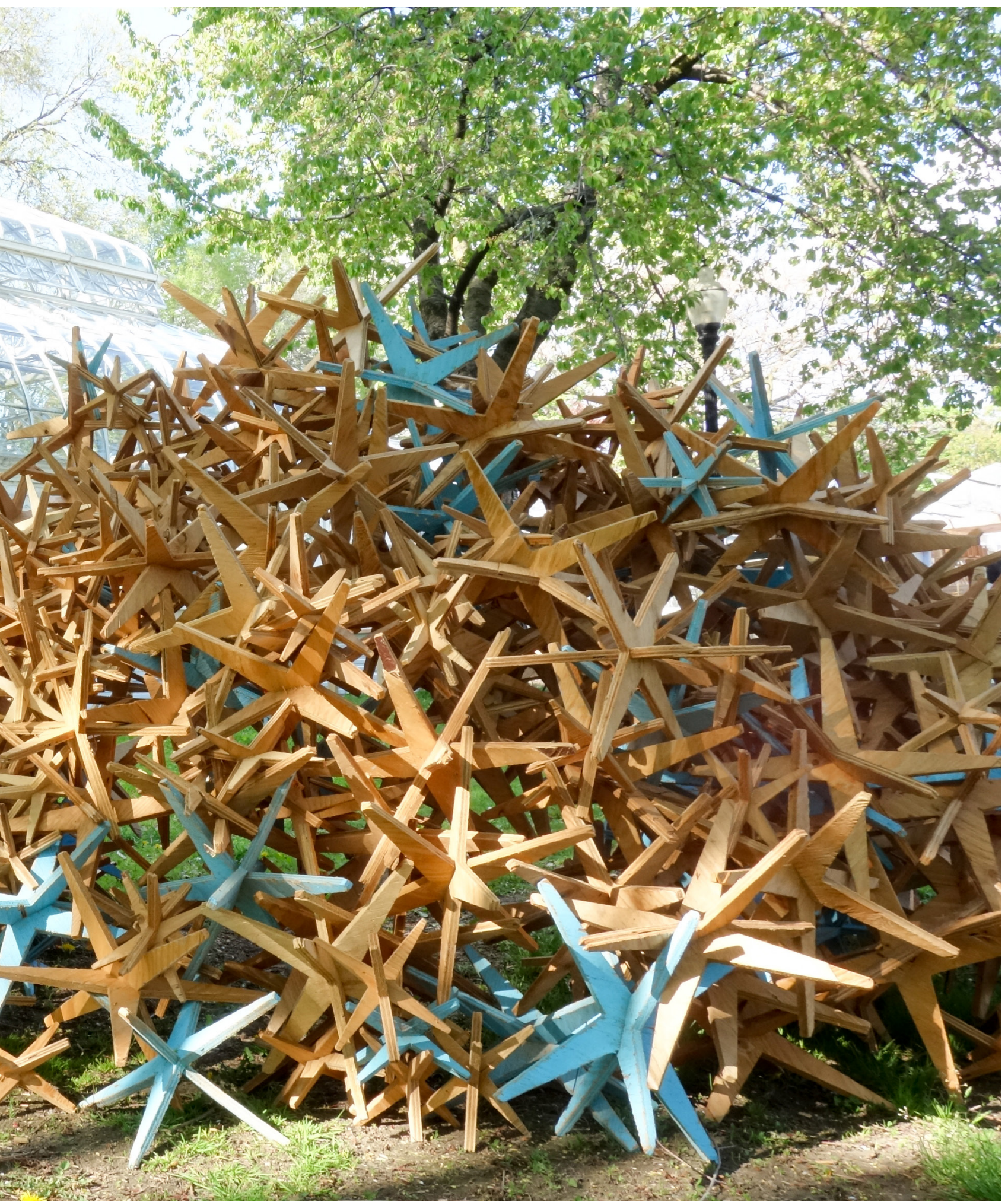




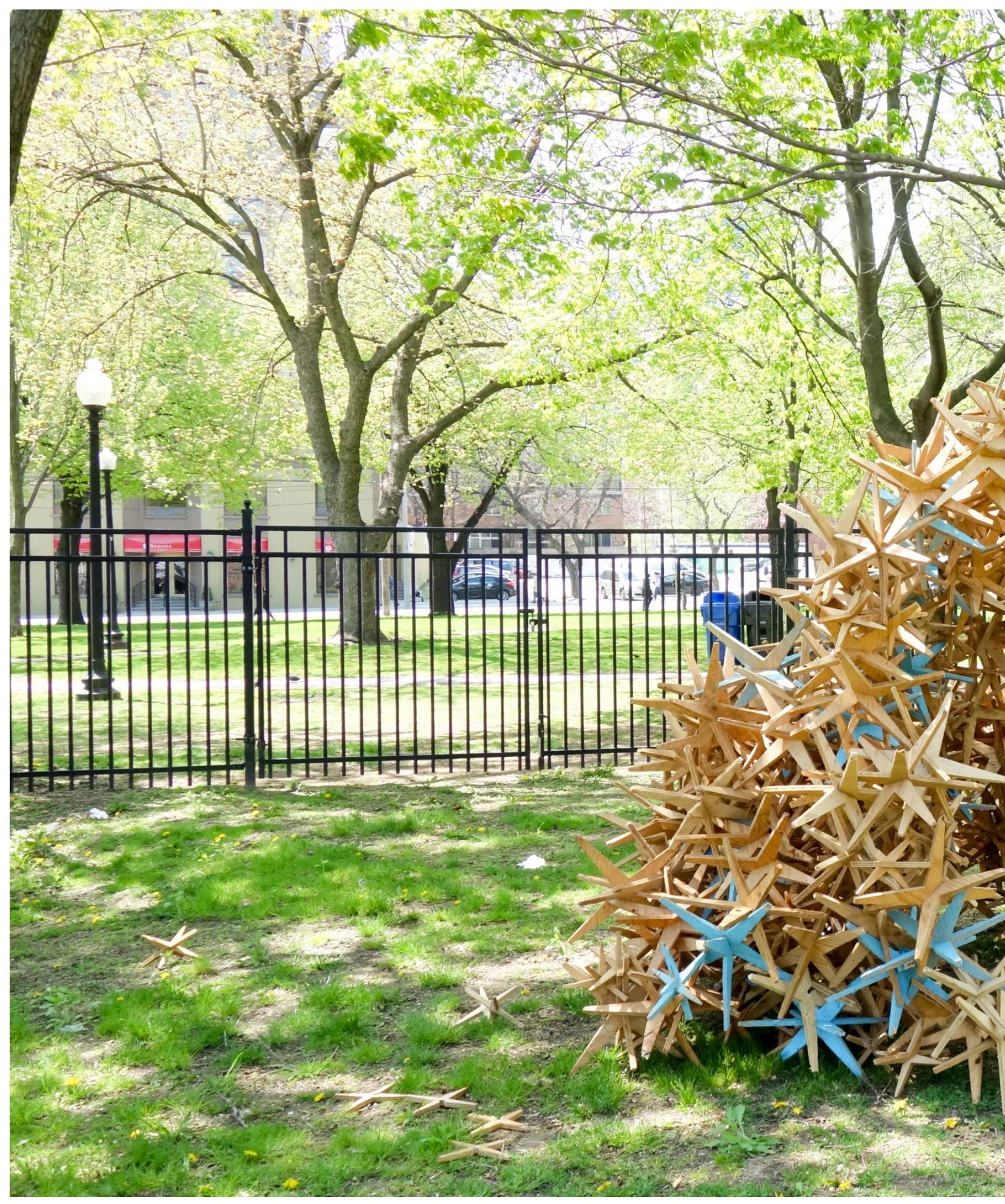




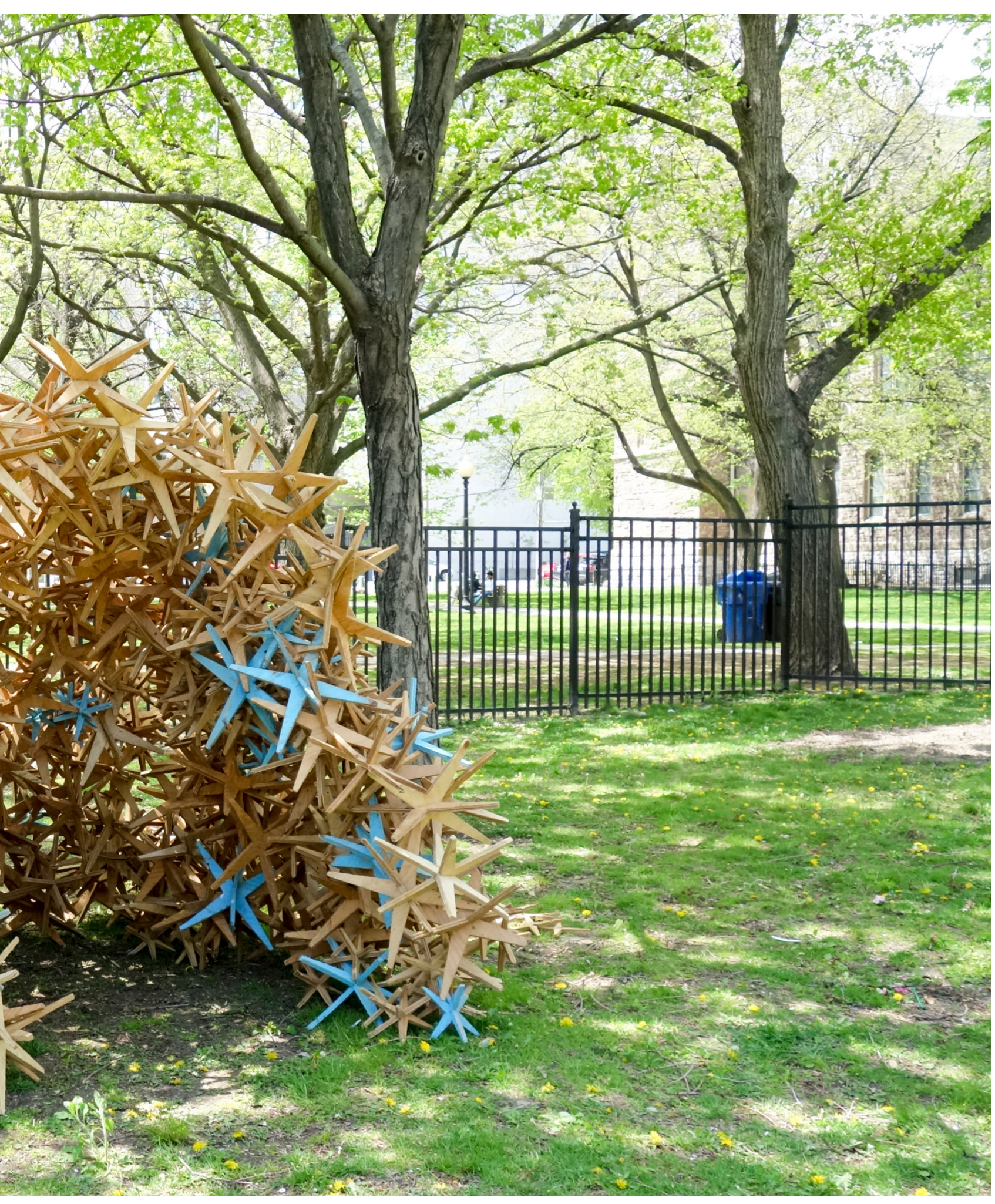


Appendix E: JaXX (Mako Student Innovation Award Finalist)

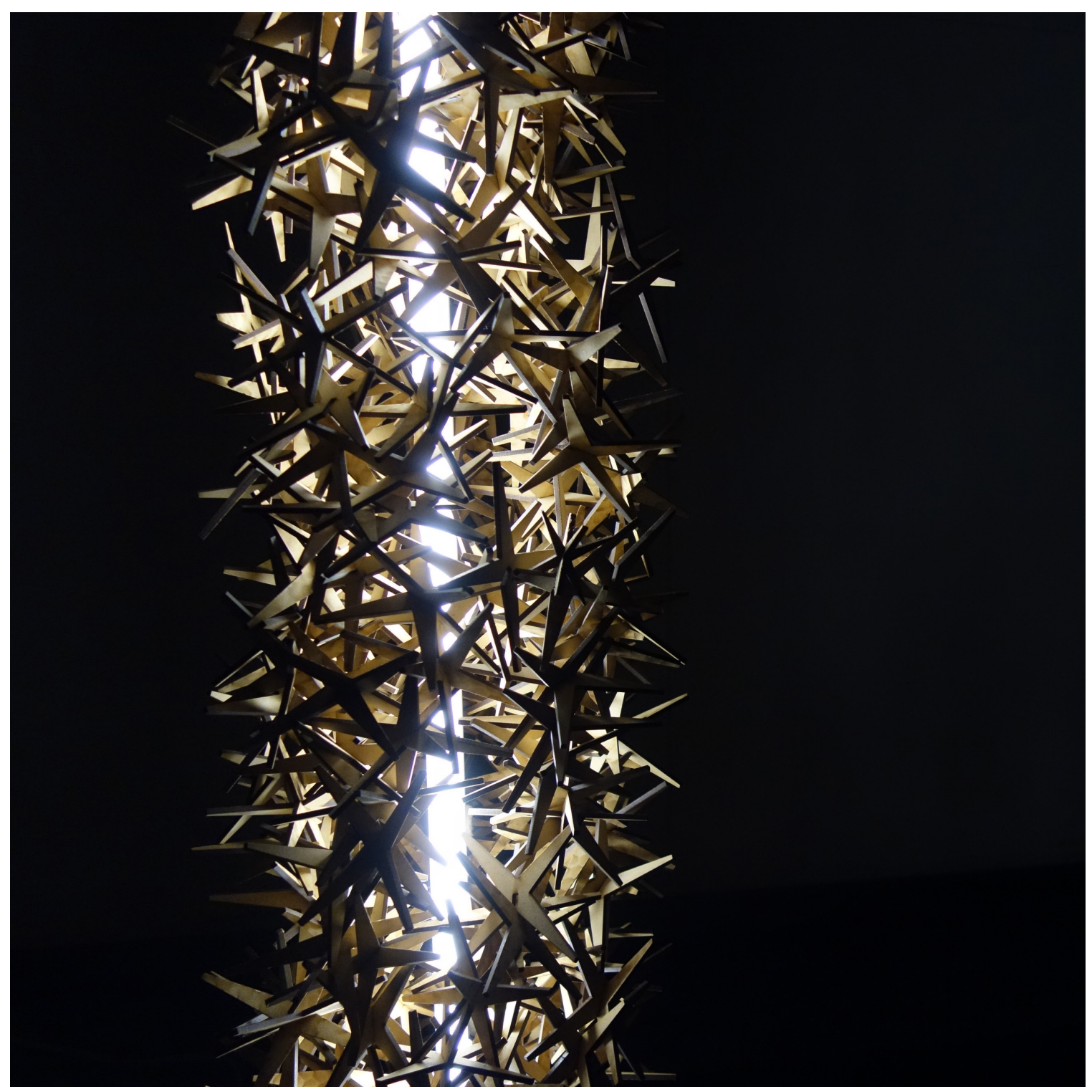

Lighting concept 


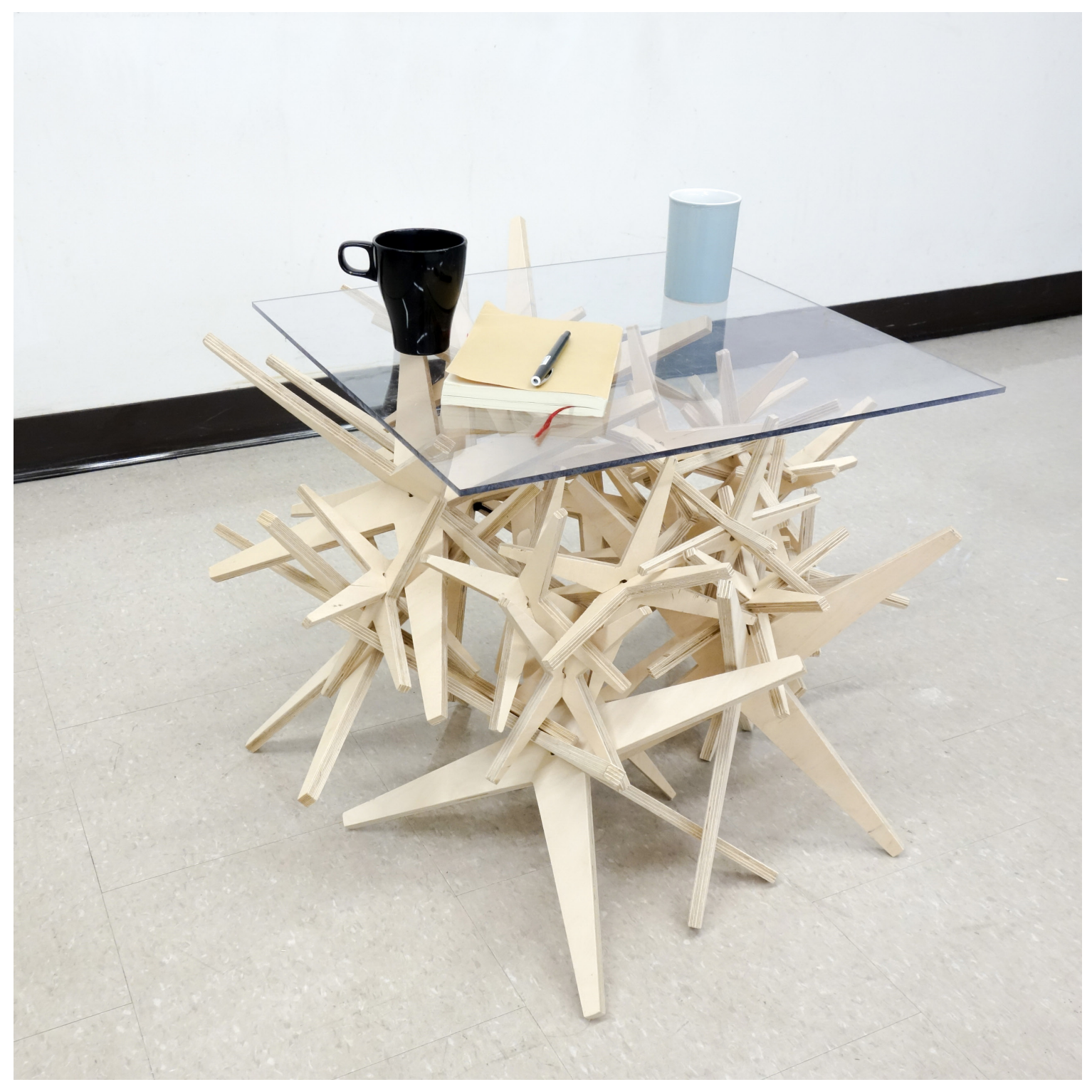

Furniture concept 


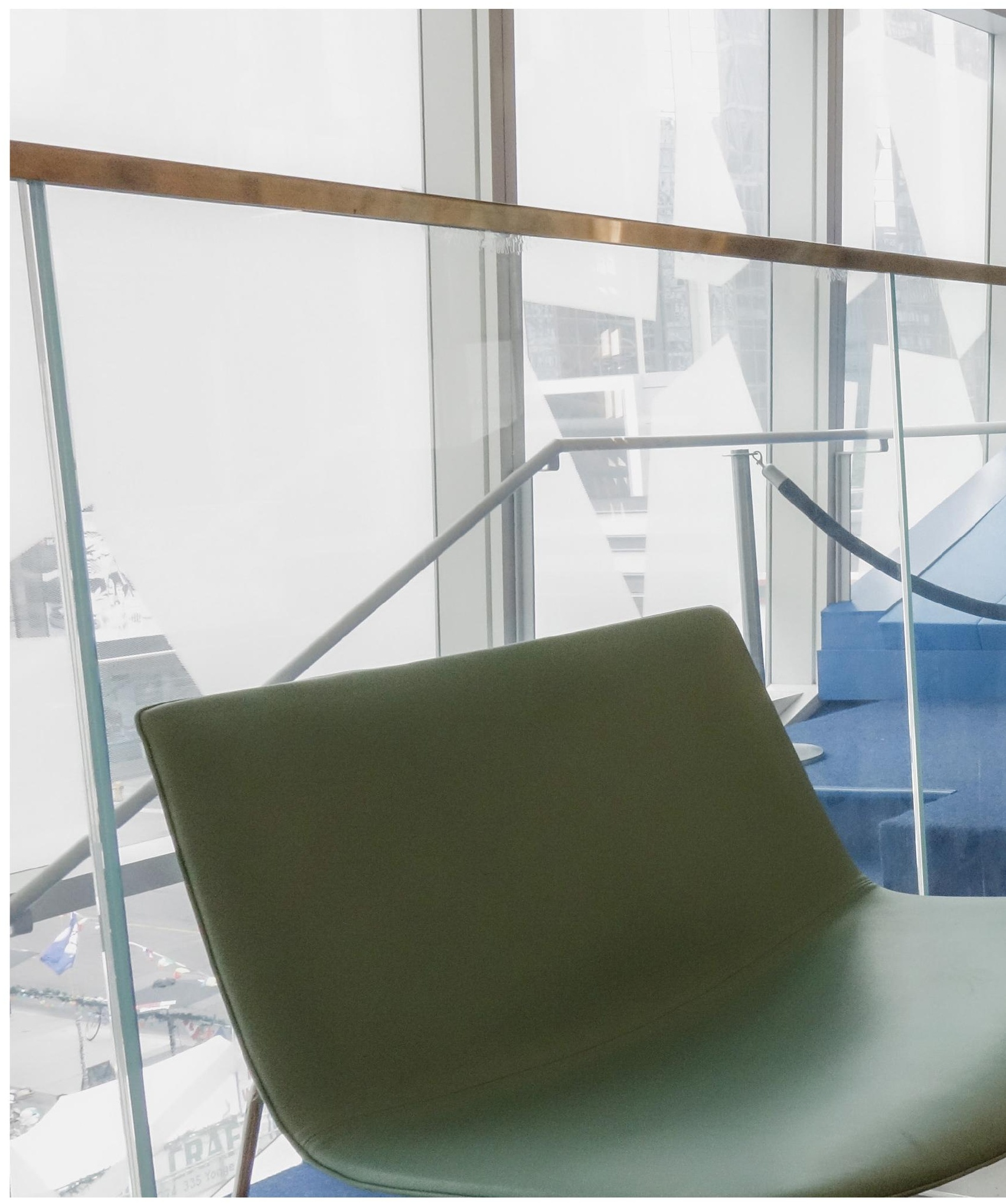

Multi-functional spatial component

208 Architecture at the Edge of Chaos 


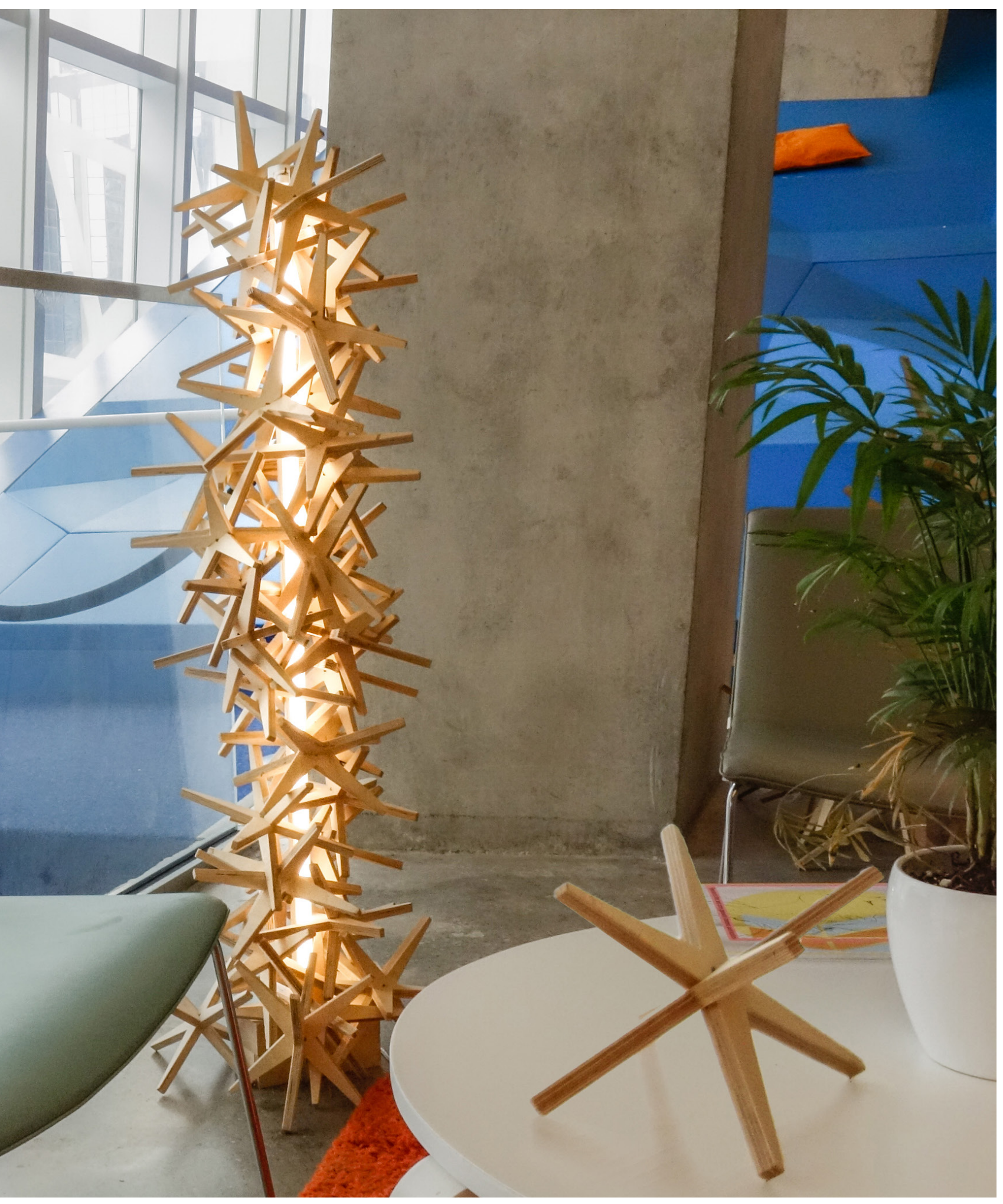


Appendix F: Speculations and Explorations

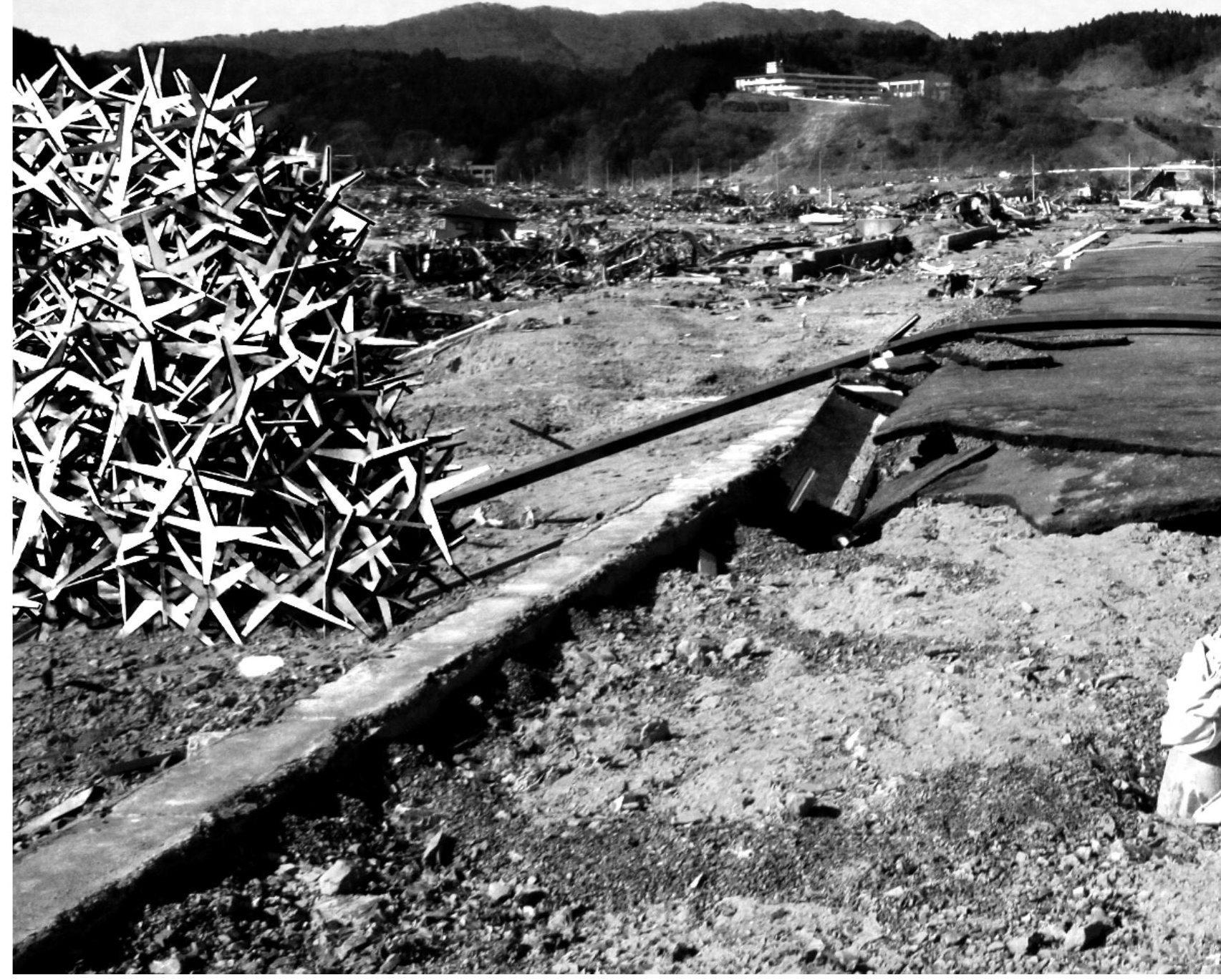

The material is able to adapt to the unknown and harsh conditions 


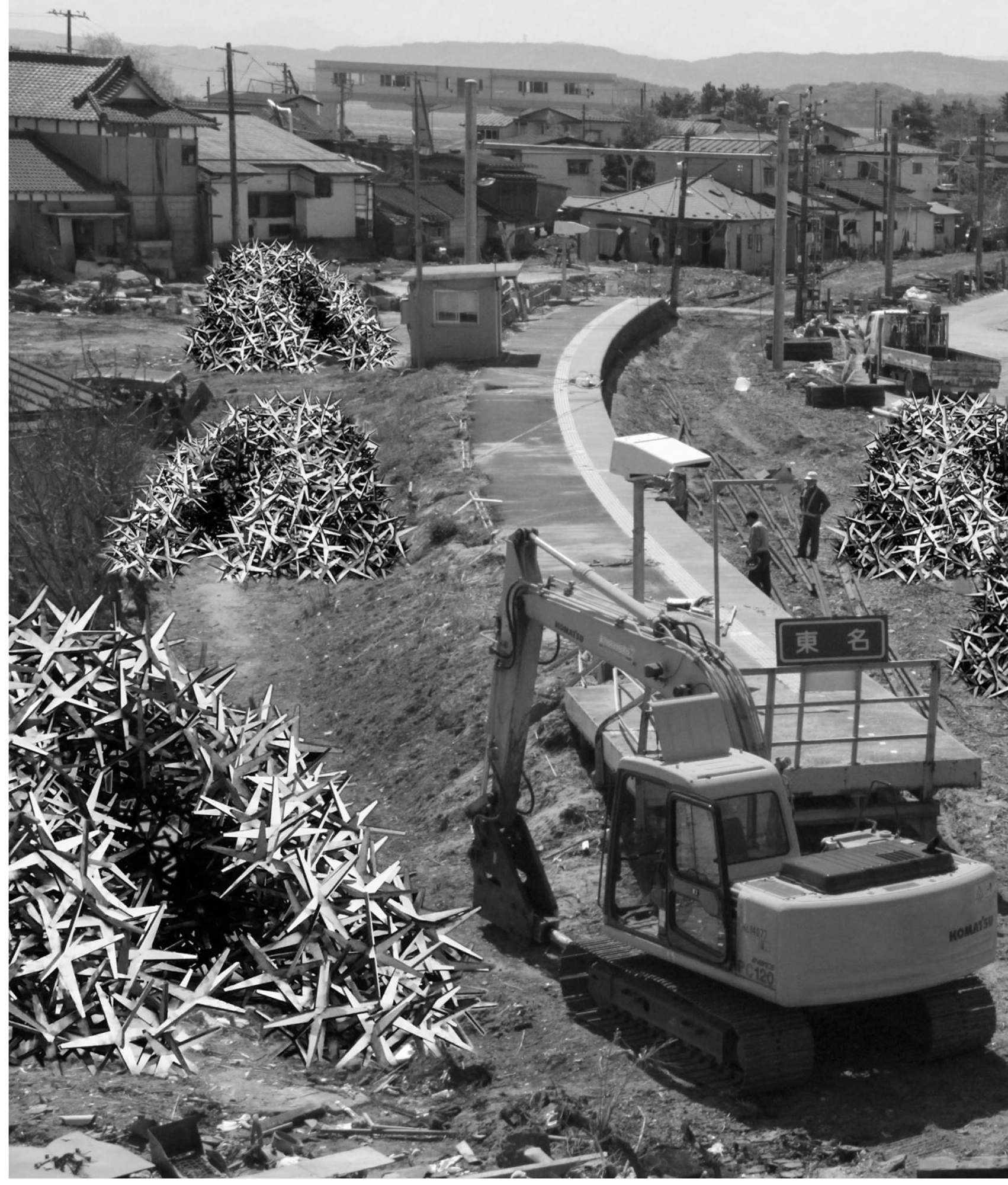

The system is valued for its rapid deployment and ease of construction

212 Architecture at the Edge of Chaos 


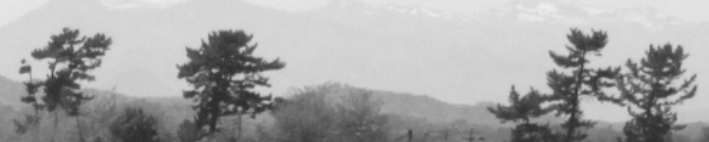

Wha $\mathrm{u}^{\mathrm{k}} \mathrm{k}$ $5+\frac{1}{2}$

$x^{2}+2 x+2$,

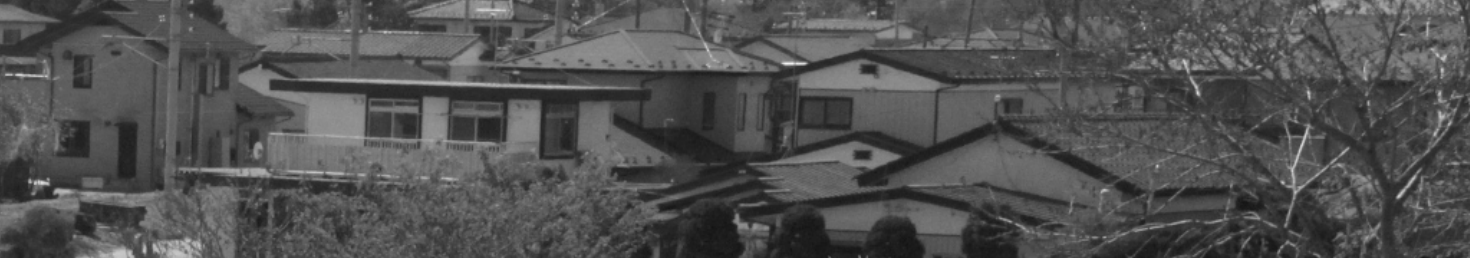

\section{0}
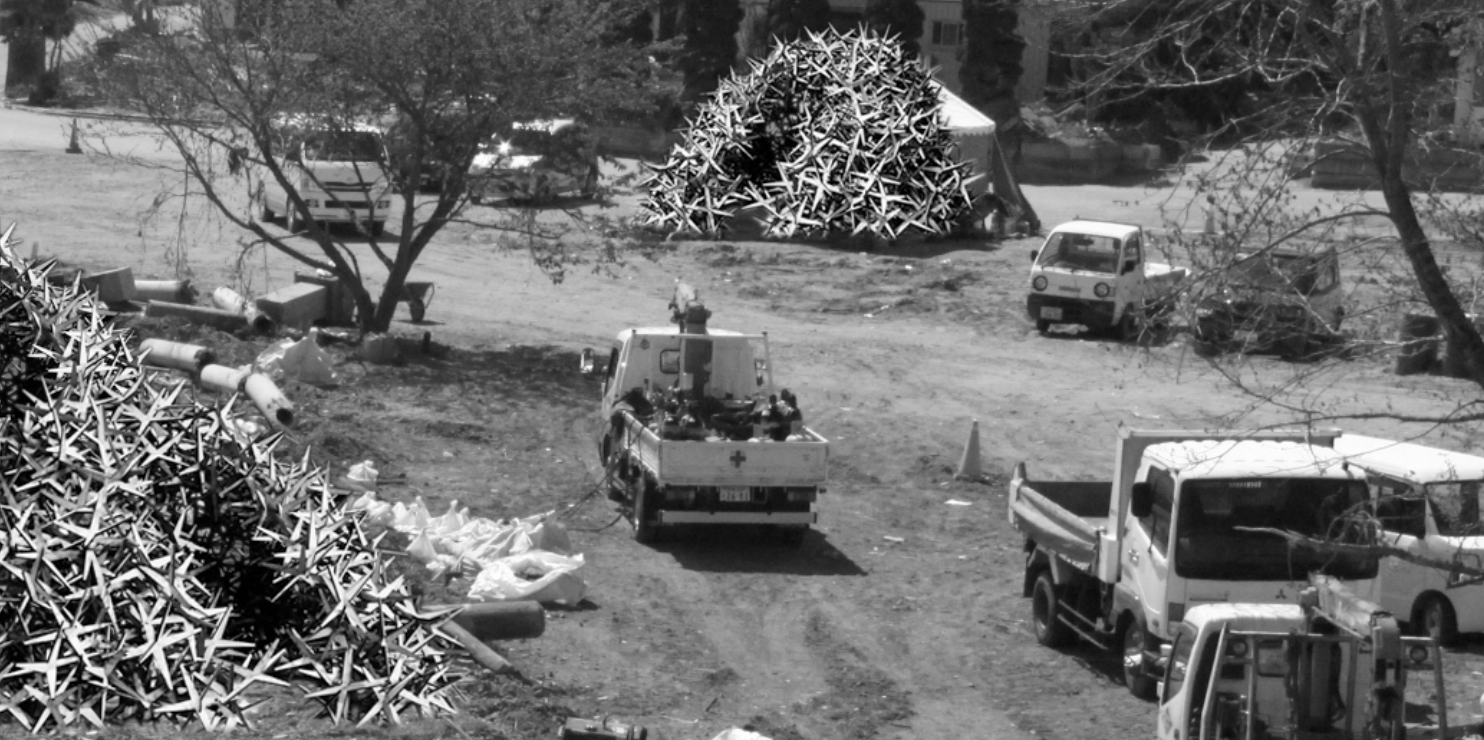

1) $\frac{1}{2}$
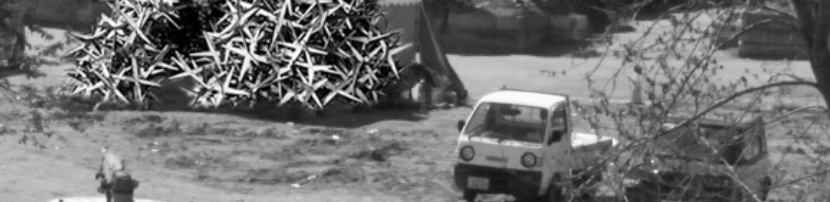

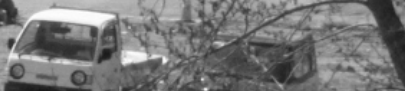

$-7,-1$

D.f.
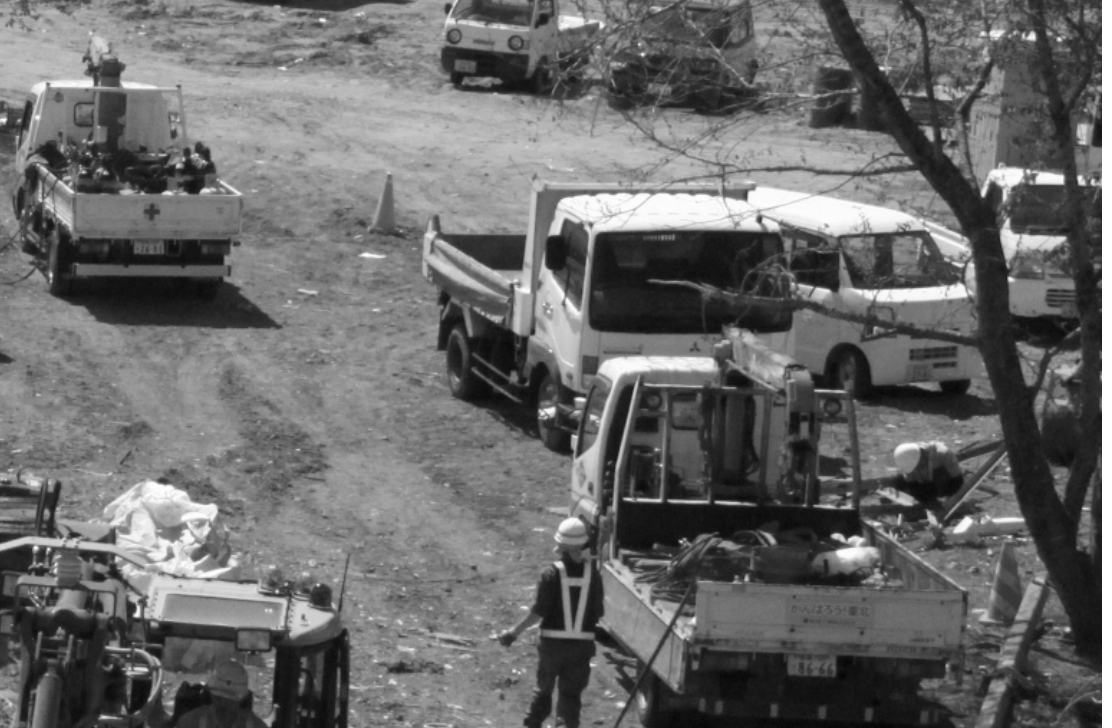


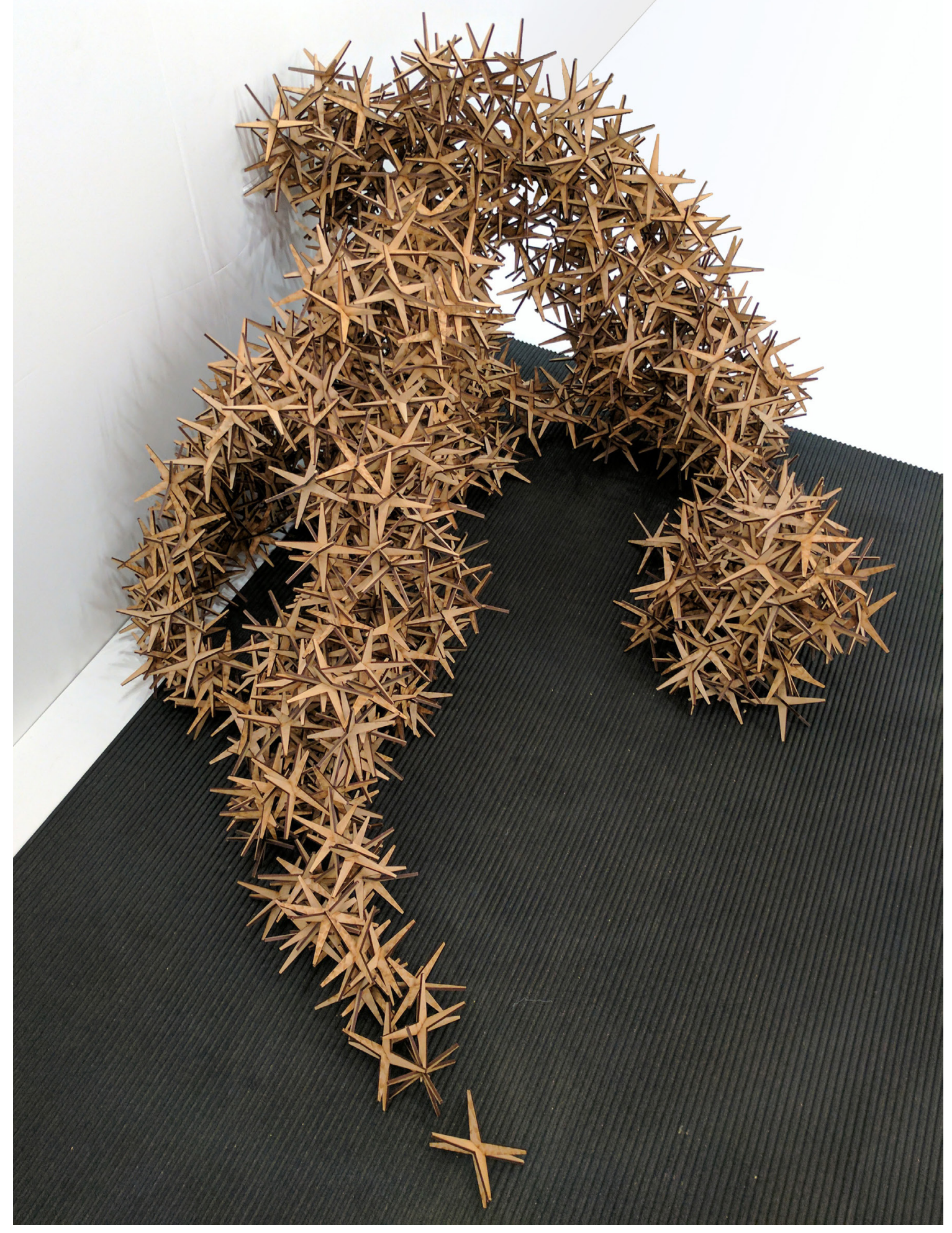

Exploration of extreme forms

214 Architecture at the Edge of Chaos 


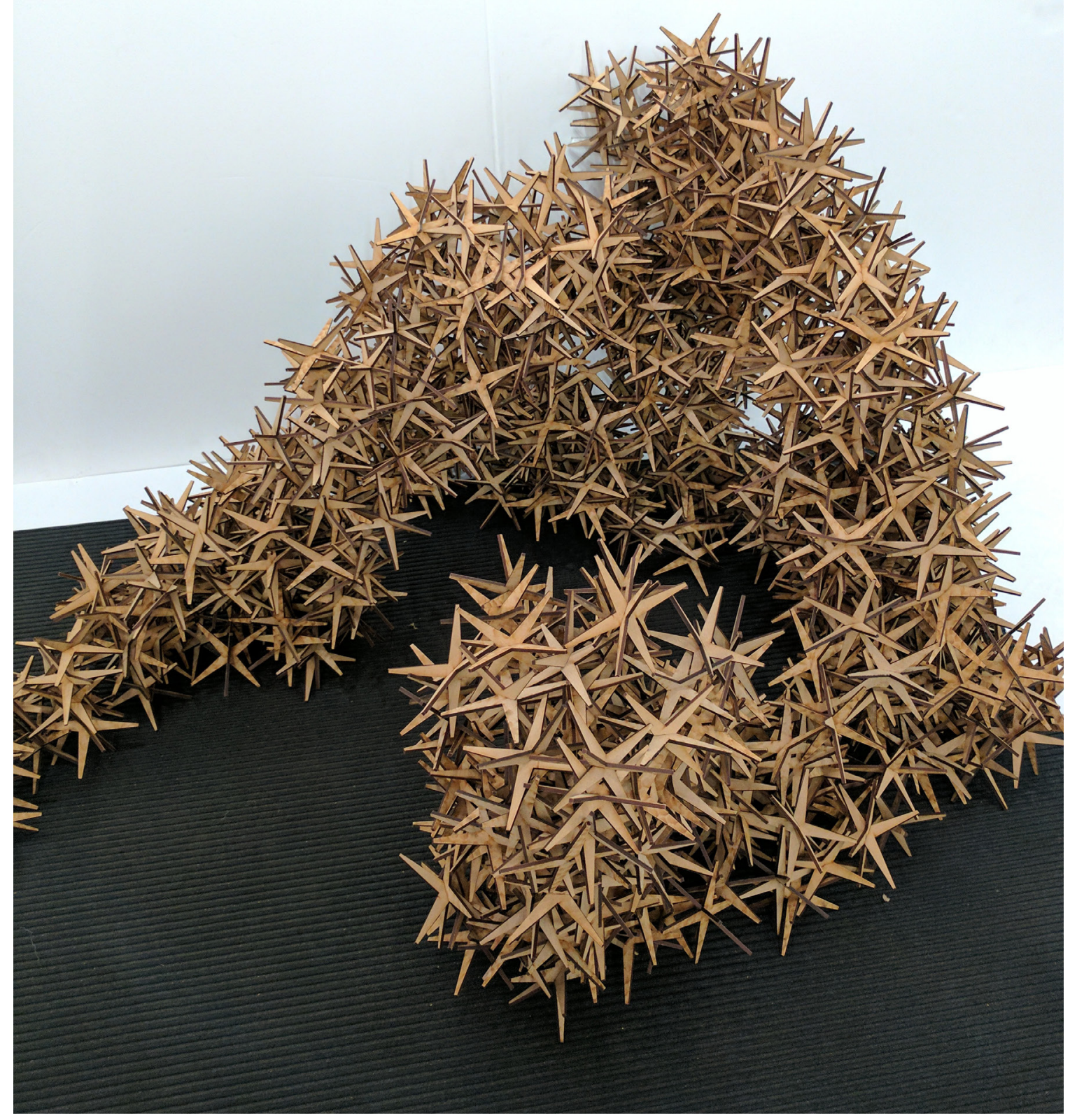

A wide range of formal possibilities 


\section{BIBLIOGRAPHY}

Aejmelaeus-Lindström, P., Willmann, J., Tibbits, S., Gramazio, F., \& Kohler, M. (2016). Jammed architectural structures: towards large-scale reversible construction. Granular Matter, 18(2), 1-12. https://doi.org/10.1007/s10035-016-0628-y

Ball, P. (1999). The self-made tapestry: pattern formation in nature. Oxford University Press.

Bauman, Z. (2012). Liquid modernity. Polity Press. Retrieved from http://www.ryerson.eblib.com/ EBLWeb/patron/?target=patron\&extendedid=P_1245694_0

Bhatia, N., \& Sheppard, L. (Eds.). (2013a). Bracket 2: Goes Soft. Barcelona; New York: Actar.

Bhatia, N., \& Sheppard, L. (2013b). Going Soft. In N. Bhatia \& L. Sheppard (Eds.), Bracket 2: Goes Soft. Barcelona; New York: Actar.

Constant. (1974). New Babylon [exhibition catalogue]. (J. L. Locher, Ed.). Haags Gemeetenmuseum. Retrieved from http://www.notbored.org/new-babylon.html

DeLanda, M. (1992). Nonorganic Life. In J. Crary \& S. Kwinter (Eds.), Zone 6: Incorporations (pp. 129-167). New York, NY: Zone Books.

DeLanda, M. (2004). Material Complexity. In N. Leach, D. Turnbull, \& C. Williams (Eds.), Digital tectonics. Wiley-Academy.

Dierichs, K., \& Menges, A. (2012a). Aggregate Architectures: Observing and Designing with Changeable Material Systems in Architecture. In X. Costa \& M. Thorne (Eds.), [Changes architecture education practices]: 2012 ACSA international conference. Wahington: ACSA Press.

Dierichs, K., \& Menges, A. (2012b). Functionally Graded Aggregate Structures: Digital Additive Manufacturing with Designed Granulates. In Synthetic Digital Ecologies: Proceedings of the 32nd Annual Conference of the Association for Computer Aided Design in Architecture. California College of the Arts.

Dierichs, K., \& Menges, A. (2015). Simulation of Aggregate Structures in Architecture: DistinctElement Modeling of Synthetic Non-convex Granulates. In Advances in Architectural Geometry 2014. Cham, DEU: Springer International Publishing. Retrieved from http://site.ebrary.com/ lib/alltitles/docDetail.action?docID=11003542

Dierichs, K., \& Menges, A. (2016). Towards an aggregate architecture: designed granular systems as programmable matter in architecture. Granular Matter, 18(2), 1-14. https://doi.org/10.1007/ s10035-016-0631-3 
Environment Canada. (n.d.-a). Daily Data Report for February 2016. Retrieved April 11, 2016, from http://climate.weather.gc.ca/climateData/dailydata_e.html?timeframe $=2 \&$ Prov $=$ ON $\&$ StationID $=51459 \&$ dlyRange $=2013-06-13 \mid 2016-04-09 \&$ Year=2016\&M onth $=2 \&$ Day $=1$

Environment Canada. (n.d.-b). Daily Data Report for March 2016. Retrieved April 12, 2016, from http://climate.weather.gc.ca/climateData/dailydata_e.html?timeframe=2\&Prov=ON\&StationID $=51459 \&$ dlyRange $=2013-06-13 \% 7 \mathrm{C} 2016-04-10 \&$ Year $=2016 \& \mathrm{Mo}$ nth=3\&Day=1

Environment Canada. (n.d.-c). Hourly Data Report for February 12, 2016. Retrieved April 7, 2016, from http://climate.weather.gc.ca/climateData/hourlydata_e. html?StationID=51459\&timeframe=1\&Year=2016\&Month=2\&cmdB1 =Go\&Day=12

Fernández-Galiano, L. (2000). Fire and memory: on architecture and energy. (G. Cariño, Trans.). MIT Press.

Fluid. (n.d.). Oxford Dictionaries. Retrieved from http://www.oxforddictionaries.com/definition/ english/fluid

Forty, A. (2000). Words and buildings: a vocabulary of modern architecture. Thames \& Hudson.

Frazer, J. (1995). An evolutionary architecture. London: Architectural Association.

GC Prostho Museum Research Center / Kengo Kuma \& Associates. (2012, January 16). Retrieved April 17, 2016, from http://www.archdaily.com/199442/ gc-prostho-museum-research-center-kengo-kuma-associates/

Heathcote, E. (2013, August 9). Toytown and the city. Financial Times. Retrieved from http://www. ft.com/cms/s/2/ab0f3f42-feb3-11e2-b9b0-00144feabdc0.html

Hensel, M., \& Menges, A. (2008). Aggregates. Architectural Design, 78(2), 80-87. https://doi. org/10.1002/ad.645

Heynen, H. (1999). Architecture and modernity: a critique. MIT Press.

Hill, J. (2003). Actions of architecture: architects and creative users. Routledge. Retrieved from http:// ezproxy.lib.ryerson.ca/login?url=http://lib.myilibrary.com?id=5607

Huijben, F. (2016). Vacuumatic formwork: a novel granular manufacturing technique for producing topology-optimised structures in concrete. Granular Matter, 18(2), 1-8. https://doi. org/10.1007/s10035-015-0602-0

Jaeger, H. M. (2015). Celebrating Soft Matter's 10th Anniversary: Toward jamming by design. Soft Matter, 11(1), 12-27. https://doi.org/10.1039/C4SM01923G

Joachim, M. (n.d.). Fab Tree Hab. Retrieved December 1, 2016, from http://www.terreform.org/projects_habitat_fab.html 
Kay, J. J. (2008). An Introduction to Systems Thinking. In D. Waltner-Toews, J. J. Kay, \& N.-M. E. Lister, The ecosystem approach: complexity, uncertainty, and managing for sustainability. Columbia University Press. Retrieved from http:/www.ryerson.eblib.com/EBLWeb/ patron/?target=patron\&extendedid=P_896784_0

Keller, S., \& Jaeger, H. M. (2016). Aleatory Architectures. Granular Matter, 18(2), 1-11. https://doi. org/10.1007/s10035-016-0629-x

Kendall, S. H., \& Teicher, J. (2010). Residential Open Building. Routledge.

Kwinter, S. (1993). Soft Systems. In B. Boigen (Ed.), Culture Lab. New York.

Kwinter, S. (2001). Architectures of time: toward a theory of the event in modernist culture. MIT Press.

Lambert, L. (2014, December 8). SPICULATION (2008). Retrieved from http://www.leopoldlambert. net/2014/12/08/spiculation-2008/

Lefebvre, H. (1991). The production of space. (D. Nicholson-Smith, Trans.). Blackwell.

Lucien, M. (2015, October 5). "Rock Print" at the forefront of architectural innovation. Retrieved November 20, 2015, from https://www.ethz.ch/en/news-and-events/eth-news/news/2015/10/ rock-print-at-the-forefront-of-architectural-innovation.html

Manolopoulou, Y. (2013). Architectures of chance. Farnham, Surrey: Ashgate Publishing Limited.

Mathews, S. (2005). The Fun Palace: Cedric Price's experiment in architecture and technology. Technoetic Arts: A Journal of Speculative Research, 3(2), 73-91. https://doi.org/10.1386/ tear.3.2.73/1

Mathews, S. (2006). The Fun Palace as virtual architecture: Cedric Price and the practices of indeterminacy. Journal of Architectural Education, 59(3), 39-48.

Mathews, S. (2007). From agit-prop to free space: the architecture of Cedric Price. London: Black Dog Pub. Ltd.

Moe, K. (2013). Convergence: an architectural agenda for energy. Routledge, Taylor \& Francis Group.

Murphy, K. A., Reiser, N., Choksy, D., Singer, C. E., \& Jaeger, H. M. (2016). Freestanding loadbearing structures with Z-shaped particles. Granular Matter, 18(2), 1-9. https://doi.org/10.1007/ s10035-015-0600-2

Negroponte, N. (1975). Soft Architecture Machines. The MIT Press.

Pask, G. (1968). An approach to cybernetics. London: Hutchinson.

Pask, G. (1969). The Architectural Relevance of Cybernetics. Architectural Design, 39, 494-496.

Patrick, R., Nicodemi, M., Delannay, R., Ribière, P., \& Bideau, D. (2005). Slow relaxation and 
compaction of granular systems. Nature Materials, 4(2), 121-8. https://doi.org/http://dx.doi. org.ezproxy.lib.ryerson.ca/10.1038/nmat 1300

Pearce, P. (1978). Structure in nature is a strategy for design. MIT Press.

Rhodes, M. (2016, January 11). Japan's Minimalist Version of Lego Is Actually Awesome. Retrieved December 1, 2016, from https://www.wired.com/2016/01/ japans-minimalist-version-of-lego-is-actually-awesome/

Sadler, S. (1999). The Situationist City. MIT.

Sadler, S. (2005). Archigram : Architecture Without Architecture. Cambridge, US: MIT Press. Retrieved from http://site.ebrary.com/lib/alltitles/docDetail.action?docID=10173594

Schneider, T., \& Till, J. (2005). Flexible housing: the means to the end. Arq: Architectural Research Quarterly, 9(3-4), 287-296. https://doi.org/10.1017/S1359135505000345

Solà-Morales, I. de. (1998). Liquid Architecture. In C. C. Davidson \& Anyone Corporation (Eds.), Anyhow (pp. 36-43). New York : Cambridge, Mass: Anyone Corp. ; MIT Press.

Steiner, H. (2005). The forces of matter. The Journal of Architecture, 10(1), 91-109. https://doi. org/10.1080/13602360500063386

The Bartlett School of Architecture, UCL. (n.d.). Bloom: Distributed Urban Game. Retrieved April 17, 2016, from https://www.bartlett.ucl.ac.uk/architecture/research/projects/bloom

Till, J. (2009). Architecture Depends. MIT Press.

Tsubaki, K. (2012). Tumbling Units: Tectonics of indeterminate extension. In G. P. Borden \& M. Meredith (Eds.), Matter: material processes in architectural production (pp. 187-203). Routledge.

Weinstock, M. (2010). The architecture of emergence: the evolution of form in nature and civilisation. Wiley.

Wigley, M. (1998). Constant's New Babylon: The Hyper-architecture of Desire. Rotterdam: 010 Publishers.

Winter Stations Design Competition 2016. (2015). Retrieved April 5, 2016, from http://winterstations.com/

Zhao, Y., Liu, K., Zheng, M., Barés, J., Dierichs, K., Menges, A., \& Behringer, R. P. (2016). Packings of 3D stars: stability and structure. Granular Matter, 18(2), 1-8. https://doi.org/10.1007/ s10035-016-0606-4 


\section{Works Consulted}

Dierichs, K., \& Menges, A. (2012). Aggregate Structures: Material and Machine Computation of Designed Granular Substances. Architectural Design, 82(2), 74-81. https://doi.org/10.1002/ ad. 1382

Dierichs, K., \& Menges, A. (2012). Material and Machine Computation of Designed Granular Matter: Rigid-Body Dynamics Simulations as a Design Tool for Robotically-Poured Aggregate Structures Consisting of Polygonal Concave Particles. In Physical Digitality: Proceedings of the 30th eCAADe Conference (Vol. 2, pp. 711-719). Prague, Czech Republic: Czech Technical University in Prague, Faculty of Architecture.

Dierichs, K., \& Menges, A. (2013). Aggregate Architecture: Simulation Models for Synthetic Nonconvex Granulates. In Adaptive Architecture [Proceedings of the 33rd Annual Conference of the Association for Computer Aided Design in Architecture (ACADIA) (pp. 301-310). Cambridge, Ontario, Canada.

Dierichs, K., \& Menges, A. (2015). Granular Morphologies: Programming Material Behaviour with Designed Aggregates. Architectural Design, 85(5), 86-91. https://doi.org/10.1002/ad.1959

DeLanda, M. (2015). The New Materiality. Architectural Design, 85(5), 16-21. https://doi. org/10.1002/ad.1948

Bashawri, A., Garrity, S., \& Moodley, K. (2014). An Overview of the Design of Disaster Relief Shelters. Procedia Economics and Finance, 18, 924-931. https://doi.org/10.1016/ S2212-5671(14)01019-3

Charlesworth, E. R. (2014). Humanitarian architecture. Routledge.

Fox, M. (2014). Catching up with the Past: A Small Contribution to a Long History of Interactive Environments. Footprint, 4(1), 5-18. https://doi.org/10.7480/footprint.4.1.716

Gleiniger, A., \& Vrachliotis, G. (2008). Complexity. Birkhäuser.

Habraken, N. J. (1972). Supports: an alternative to mass housing. Praeger.

Habraken, N. J., \& Teicher, J. (1998). The Structure of the Ordinary: Form and Control in the Built Environment. MIT Press.

Hertzberger, H. (2014). Polyvalence: The Competence of Form and Space with Regard to Different Interpretations. Architectural Design, 84(5), 106-113. https://doi.org/10.1002/ad.1816

Kahn, L. (2003). Louis Kahn Essential Texts. (R. Twombly, Ed.). New York: WW Norton.

Kolarevic, B. (2009). Exploring Architecture of Change. In reForm() - Building a Better Tomorrow: Proceedings of the 29th Annual Conference of the Association for Computer Aided Design in Architecture (ACADIA). The School of the Art Institute of Chicago. 
Kolarevic, B., \& Parlac, V. (Eds.). (2015). Building Dynamics: Exploring Architecture of Change. Abingdon, Oxon; New York, NY: Routledge.

Negroponte, N. (1970). Architecture machine. MIT Press.

Reiser, J., \& Umemoto, N. (2006). Atlas of novel tectonics. Princeton Architectural Press.

Schön, D. A. (1983). The reflective practitioner: how professionals think in action. Basic Books.

Slavinsky, D. (2011). Authorship at Risk: The Role of the Architect. Architectural Design, 81(2), 90-99. https://doi.org/10.1002/ad.1216 


